\title{
A sharp compactness theorem for genus-one pseudo-holomorphic maps
}

\author{
AleKSEY Zinger
}

For every compact almost Kahler manifold $(X, \omega, J)$ and an integral second homology class $A$, we describe a natural closed subspace $\overline{\mathfrak{M}}_{1, k}^{0}(X, A ; J)$ of the moduli space $\overline{\mathfrak{M}}_{1, k}(X, A ; J)$ of stable $J$-holomorphic genus-one maps such that $\overline{\mathfrak{M}}_{1, k}^{0}(X, A ; J)$ contains all stable maps with smooth domains. If $\left(\mathbb{P}^{n}, \omega, J_{0}\right)$ is the standard complex projective space, $\overline{\mathfrak{M}}_{1, k}^{0}\left(\mathbb{P}^{n}, A ; J_{0}\right)$ is an irreducible component of $\overline{\mathfrak{M}}_{1, k}\left(\mathbb{P}^{n}, A ; J_{0}\right)$. We also show that if an almost complex structure $J$ on $\mathbb{P}^{n}$ is sufficiently close to $J_{0}$, the structure of the space $\overline{\mathfrak{M}}_{1, k}^{0}\left(\mathbb{P}^{n}, A ; J\right)$ is similar to that of $\overline{\mathfrak{M}}_{1, k}^{0}\left(\mathbb{P}^{n}, A ; J_{0}\right)$. This paper's compactness and structure theorems lead to new invariants for some symplectic manifolds, which are generalized to arbitrary symplectic manifolds in a separate paper. Relatedly, the smaller moduli space $\overline{\mathfrak{M}}_{1, k}^{0}(X, A ; J)$ is useful for computing the genus-one Gromov-Witten invariants, which arise from the larger moduli space $\overline{\mathfrak{M}}_{1, k}(X, A ; J)$.

\section{D20; 53D99}

\section{Introduction}

\subsection{Background and motivation}

Gromov-Witten invariants of symplectic manifolds have been a subject of much research in the past decade, as they play a prominent role in both symplectic topology and theoretical physics. In order to define $\mathrm{GW}$-invariants of a compact symplectic manifold $(X, \omega)$, one fixes an almost complex structure $J$ on $X$, which is compatible with $\omega$ or at least is tamed by $\omega$. For each class $A$ in $H_{2}(X ; \mathbb{Z})$ and a pair $(g, k)$ of nonnegative integers, let $\overline{\mathfrak{M}}_{g, k}(X, A ; J)$ be the moduli space of (equivalence classes of) stable $J$-holomorphic maps from genus- $g$ Riemann surfaces with $k$ marked points in the homology class $A$. The expected, or virtual, dimension of this moduli space is given by

$$
\operatorname{dim}_{g, k}(X, A) \equiv \operatorname{dim}^{\text {vir }} \overline{\mathfrak{M}}_{g, k}(X, A ; J)=2\left(\left\langle c_{1}(T X), A\right\rangle+(n-3)(1-g)+k\right),
$$

if the real dimension of $X$ is $2 n$. While in general $\overline{\mathfrak{M}}_{g, k}(X, A ; J)$ is not a smooth manifold, or even a variety, Fukaya-Ono [4], Li-Tian [6], and in the algebraic case 
Behrend-Fantechi [1] show that $\overline{\mathfrak{M}}_{g, k}(X, A ; J)$ determines a rational homology class of $\operatorname{dimension}_{\operatorname{dim}_{g, k}}(X, A)$. In turn, this virtual fundamental class of $\overline{\mathfrak{M}}_{g, k}(X, A ; J)$ is used to define GW-invariants of $(X, \omega)$.

We denote by $\mathfrak{M}_{g, k}^{0}(X, A ; J)$ the subspace of $\overline{\mathfrak{M}}_{g, k}(X, A ; J)$ consisting of the stable maps $[\mathcal{C}, u]$ such that the domain $\mathcal{C}$ is a smooth Riemann surface. If $\left(\mathbb{P}^{n}, \omega ; J_{0}\right)$ is the $n$-dimensional complex projective space with the standard Kahler structure and $\ell$ is the homology class of a complex line in $\mathbb{P}^{n}$,

$$
\mathfrak{M}_{g, k}^{0}\left(\mathbb{P}^{n}, d\right) \equiv \mathfrak{M}_{g, k}^{0}\left(\mathbb{P}^{n}, d \ell ; J_{0}\right)
$$

is in fact a smooth orbifold of dimension $\operatorname{dim}_{g, k}\left(\mathbb{P}^{n}, d \ell\right)$, at least for $d \geq 2 g-1$. In addition, from the point of view of algebraic geometry, $\overline{\mathfrak{M}}_{0, k}\left(\mathbb{P}^{n}, d\right)$ is an irreducible algebraic orbivariety of dimension $\operatorname{dim}_{0, k}\left(\mathbb{P}^{n}, d \ell\right) / 2$. From the point of view of symplectic topology, $\overline{\mathfrak{M}}_{0, k}\left(\mathbb{P}^{n}, d\right)$ is a compact topological orbifold stratified by smooth orbifolds of even dimensions and $\mathfrak{M}_{0, k}^{0}\left(\mathbb{P}^{n}, d\right)$ is the main stratum of $\overline{\mathfrak{M}}_{0, k}\left(\mathbb{P}^{n}, d\right)$. In particular, $\mathfrak{M}_{0, k}^{0}\left(\mathbb{P}^{n}, d\right)$ is a dense open subset of $\overline{\mathfrak{M}}_{0, k}\left(\mathbb{P}^{n}, d\right)$.

If $g \geq 1$, none of these additional properties holds even for $\left(\mathbb{P}^{n}, \omega, J_{0}\right)$. For example, the moduli space $\overline{\mathfrak{M}}_{1, k}\left(\mathbb{P}^{n}, d\right)$ has many irreducible components of various dimensions. One of these components contains $\mathfrak{M}_{1, k}^{0}\left(\mathbb{P}^{n}, d\right)$; we denote this component by $\overline{\mathfrak{M}}_{1, k}^{0}\left(\mathbb{P}^{n}, d\right)$. In other words, $\overline{\mathfrak{M}}_{1, k}^{0}\left(\mathbb{P}^{n}, d\right)$ is the closure of $\mathfrak{M}_{1, k}^{0}\left(\mathbb{P}^{n}, d\right)$ in $\overline{\mathfrak{M}}_{1, k}\left(\mathbb{P}^{n}, d\right)$. The remaining components of $\overline{\mathfrak{M}}_{1, k}\left(\mathbb{P}^{n}, d\right)$ can be described as follows. If $m$ is a positive integer, let $\mathfrak{M}_{1, k}^{m}\left(\mathbb{P}^{n}, d\right)$ be the subset of $\overline{\mathfrak{M}}_{1, k}\left(\mathbb{P}^{n}, d\right)$ consisting of the stable maps $[\mathcal{C}, u]$ such that $\mathcal{C}$ is an elliptic curve $E$ with $m$ rational components attached directly to $E,\left.u\right|_{E}$ is constant, and the restriction of $u$ to each rational component is nonconstant. Figure 1 shows the domain of an element of $\mathfrak{M}_{1, k}^{3}\left(\mathbb{P}^{n}, d\right)$, from the points of view of symplectic topology and of algebraic geometry. In the first diagram, each shaded disc represents a sphere; the integer next to each rational component $\mathcal{C}_{i}$ indicates the degree of $\left.u\right|_{\mathcal{C}_{i}}$. In the second diagram, the components of $\mathcal{C}$ are represented by curves, and the pair of integers next to each component $\mathcal{C}_{i}$ shows the genus of $\mathcal{C}_{i}$ and the degree of $\left.u\right|_{\mathcal{C}_{i}}$. We denote by $\overline{\mathfrak{M}}_{1, k}^{m}\left(\mathbb{P}^{n}, d\right)$ the closure of $\mathfrak{M}_{1, k}^{m}\left(\mathbb{P}^{n}, d\right)$ in $\overline{\mathfrak{M}}_{1, k}\left(\mathbb{P}^{n}, d\right)$. The space $\overline{\mathfrak{M}}_{1, k}^{m}\left(\mathbb{P}^{n}, d\right)$ has a number of irreducible components. These components are indexed by the splittings of the degree $d$ into $m$ positive integers and by the distributions of the $k$ marked points between the $m+1$ components of the domain. However, all of these components are algebraic orbivarieties of dimension, both expected and actual,

$$
\begin{aligned}
\operatorname{dim}_{1, k}^{m}\left(\mathbb{P}^{n}, d \ell\right) \equiv \operatorname{dim} \overline{\mathfrak{M}}_{1, k}^{m}\left(\mathbb{P}^{n}, d\right) & =2(d(n+1)+k+n-m) \\
& =\operatorname{dim}_{1, k}\left(\mathbb{P}^{n}, d \ell\right)+2(n-m) .
\end{aligned}
$$


In particular, $\mathfrak{M}_{1, k}^{0}\left(\mathbb{P}^{n}, d\right)$ is not dense in $\overline{\mathfrak{M}}_{1, k}\left(\mathbb{P}^{n}, d\right)$. From the point of view of symplectic topology, $\overline{\mathfrak{M}}_{1, k}\left(\mathbb{P}^{n}, d\right)$ is a union of compact topological orbifolds and is stratified by smooth orbifolds of even dimensions. However, $\overline{\mathfrak{M}}_{1, k}\left(\mathbb{P}^{n}, d\right)$ contains several main strata, and some of them are of dimension larger than $\operatorname{dim}_{1, k}\left(\mathbb{P}^{n}, d \ell\right)$.

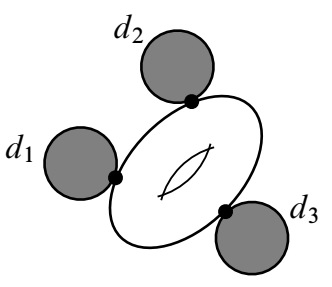

$$
d_{1}+d_{2}+d_{3}=d, \quad d_{1}, d_{2}, d_{3}>0
$$

Figure 1: The domain of an element of $\mathfrak{M}_{1, k}^{3}\left(\mathbb{P}^{n}, d\right)$

The above example shows that $\overline{\mathfrak{M}}_{1, k}^{0}\left(\mathbb{P}^{n}, d\right)$ is a true compactification of the moduli space $\mathfrak{M}_{1, k}^{0}\left(\mathbb{P}^{n}, d\right)$, while $\overline{\mathfrak{M}}_{1, k}\left(\mathbb{P}^{n}, d\right)$ is simply a compact space containing $\mathfrak{M}_{1, k}^{0}\left(\mathbb{P}^{n}, d\right)$, albeit one with a nice obstruction theory. One can view $\overline{\mathfrak{M}}_{1, k}\left(\mathbb{P}^{n}, d\right)$ as a geometric-genus compactification of $\mathfrak{M}_{1, k}^{0}\left(\mathbb{P}^{n}, d\right)$ and its subspace $\overline{\mathfrak{M}}_{1, k}^{0}\left(\mathbb{P}^{n}, d\right)$ as an arithmetic-genus compactification. Since the beginning of the Gromov-Witten theory, it has been believed, or at least considered feasible, that an analogue of $\overline{\mathfrak{M}}_{1, k}^{0}\left(\mathbb{P}^{n}, d\right)$ can be defined for every compact almost Kahler manifold $(X, \omega, J)$, positive genus $g$, and nonzero homology class $A \in H_{2}(X ; \mathbb{Z})$. In this paper, we show that this is indeed the case if $g=1$.

We describe an analogue $\overline{\mathfrak{M}}_{1, k}^{0}(X, A ; J)$ of the subspace $\overline{\mathfrak{M}}_{1, k}^{0}\left(\mathbb{P}^{n}, d\right)$ of $\overline{\mathfrak{M}}_{1, k}\left(\mathbb{P}^{n}, d\right)$ for every compact almost Kahler manifold $(X, \omega, J)$ and homology class $A \in H_{2}(X ; \mathbb{Z})$ as the subset of elements of $\overline{\mathfrak{M}}_{1, k}(X, A ; J)$ that satisfy one of two conditions. By Theorem $1.2, \overline{\mathfrak{M}}_{1, k}^{0}(X, A ; J)$ is a closed subspace of $\overline{\mathfrak{M}}_{1, k}(X, A ; J)$ and thus is compact. This compactification of $\mathfrak{M}_{1, k}^{0}(X, A ; J)$ satisfies the following desirable properties:

(P1) Naturality with respect to embeddings: If $(Y, \omega, J)$ is a compact submanifold of $(X, \omega, J)$, then

$$
\overline{\mathfrak{M}}_{1, k}^{0}(Y, A ; J)=\overline{\mathfrak{M}}_{1, k}^{0}(X, A ; J) \cap \overline{\mathfrak{M}}_{1, k}(Y, A ; J) \subset \overline{\mathfrak{M}}_{1, k}(X, A ; J) .
$$

(P2) Naturality with respect to forgetful maps: If $k \geq 1$, the preimage of the subspace $\overline{\mathfrak{M}}_{1, k-1}^{0}(X, A ; J)$ under the forgetful map

$$
\overline{\mathfrak{M}}_{1, k}(X, A ; J) \longrightarrow \overline{\mathfrak{M}}_{1, k-1}(X, A ; J)
$$

is $\overline{\mathfrak{M}}_{1, k}^{0}(X, A ; J)$. 
(P3) Sharpness for regular $(X, \omega, J)$ : If $J$ satisfies the regularity conditions of Definition 1.4 , then $\overline{\mathfrak{M}}_{1, k}^{0}(X, A ; J)$ is the closure of $\mathfrak{M}_{1, k}^{0}(X, A ; J)$ in $\overline{\mathfrak{M}}_{1, k}(X, A ; J)$.

By (P1) and (P2), $\overline{\mathfrak{M}}_{1, k}^{0}(X, A ; J)$, like $\overline{\mathfrak{M}}_{1, k}(X, A ; J)$, is a natural compactification of $\mathfrak{M}_{1, k}^{0}(X, A ; J)$. By $(\mathrm{P} 3), \overline{\mathfrak{M}}_{1, k}^{0}(X, A ; J)$, in contrast to $\overline{\mathfrak{M}}_{1, k}(X, A ; J)$, is a sharp compactification of $\mathfrak{M}_{1, k}^{0}(X, A ; J)$, subject to the naturality conditions (P1) and (P2). The first two properties of $\overline{\mathfrak{M}}_{1, k}^{0}(X, A ; J)$ are immediate from Definition 1.1. The last property is part of Corollary 1.5. It is well-known that the regularity conditions of Definition 1.4 are satisfied by the standard complex structure $J_{0}$ on $\mathbb{P}^{n}$, and thus the definition of $\overline{\mathfrak{M}}_{1, k}^{0}\left(\mathbb{P}^{n}, d \ell ; J_{0}\right)$ given in Section 1.2 agrees with the description of $\overline{\mathfrak{M}}_{1, k}^{0}\left(\mathbb{P}^{n}, d\right)$ given above.

Theorem 2.3 describes, under the regularity conditions of Definition 1.1, a neighborhood of every "interesting" stratum of $\overline{\mathfrak{M}}_{1, k}^{0}(X, A ; J)$, ie a stratum consisting of genus-one maps that are constant on the principal component. In addition to implying (P3), Theorem 2.3 shows that $\overline{\mathfrak{M}}_{1, k}^{0}(X, A ; J)$ carries a rational fundamental class. It is used in Section 1.3 below to define new Gromov-Witten style intersection numbers via pseudocycles whenever $J$ is regular, mimicking the approach of McDuff-Salamon [8, Chapter 5] and Ruan-Tian [9, Section 1] to the standard GW-invariants. As the regularity requirements of Definition 1.1 are open conditions on the space of $\omega$-tame almost complex structures $J$ by Theorem 1.6, Theorem 2.3 also implies that the general topological structure of $\overline{\mathfrak{M}}_{1, k}^{0}(X, A ; J)$ remains unchanged under small changes in $J$ near a regular $J_{0}$.

The results of this paper have already found a variety of applications:

(A1) $\overline{\mathfrak{M}}_{1, k}^{0}(X, A ; J)$ gives rise to new, reduced, genus-one $\mathrm{GW}$-invariants of arbitrary symplectic manifolds (see Zinger [19]).

(A2) In contrast to the standard genus-one GW-invariants, the reduced invariants of a complete intersection and the ambient space are related as geometrically expected (see Li-Zinger [7] and Zinger [18]).

(A3) Theorem 2.3 is used in Vakil-Zinger [12] to construct a natural desingularization of $\overline{\mathfrak{M}}_{1, k}^{0}\left(\mathbb{P}^{n}, d\right)$ and thus a natural smooth compactification of the Hilbert scheme of smooth genus-one curves in $\mathbb{P}^{n}$ for $n \geq 3$.

(A4) (A1)-(A3) are used by the author in [22] to finally confirm the 1993 BershadskyCecotti-Ooguri-Vafa mirror formula [2] for the genus-one GW-invariants of a quintic threefold.

(A5) (A1)-(A3), along with Zinger [21], have made it possible to compute (standard) genus-one GW-invariants of arbitrary complete intersections. 
If it is possible to define subspaces $\overline{\mathfrak{M}}_{g, k}^{0}(X, A ; J)$ of $\overline{\mathfrak{M}}_{g, k}(X, A ; J)$ analogous to $\overline{\mathfrak{M}}_{1, k}^{0}(X, A ; J)$ for $g \geq 2$, their description is likely to be more complicated. The space $\overline{\mathfrak{M}}_{1, k}^{0}(X, A ; J)$ contains all stable maps $[\mathcal{C}, u]$ in $\overline{\mathfrak{M}}_{1, k}(X, A ; J)$ such that the restriction of $u$ to the principal component $\mathcal{C}_{P}$ is nonconstant or such that $\left.u\right|_{\mathcal{C}_{P}}$ is constant and the restrictions to the rational components satisfy a certain fairly simple degeneracy condition; see Definition 1.1. Thus, in the genus-one case the elements in $\overline{\mathfrak{M}}_{1, k}(X, A ; J)$ are split into two classes, according to their restriction to the principal component. In the genus-two case, these classes would need to be split further. For example, suppose the domain of an element $[\mathcal{C}, u]$ of $\overline{\mathfrak{M}}_{2, k}\left(\mathbb{P}^{n}, d\right)$ consists of three rational curves, $\mathcal{C}_{1}, \mathcal{C}_{2}$, and $\mathcal{C}_{3}$, such that $\mathcal{C}_{1}$ and $\mathcal{C}_{2}$ share two nodes and $\mathcal{C}_{3}$ has a node in common with $\mathcal{C}_{1}$ and $\mathcal{C}_{2}$; see Figure 2. If $\left.u\right|_{\mathcal{C}_{1}}$ and $\left.u\right|_{\mathcal{C}_{2}}$ are constant, $[\mathcal{C}, u]$ lies in the closure of $\mathfrak{M}_{2, k}^{0}\left(\mathbb{P}^{n}, d\right)$ in $\overline{\mathfrak{M}}_{2, k}\left(\mathbb{P}^{n}, d\right)$ if and only if the branches of the curve $u(\mathcal{C})=u\left(\mathcal{C}_{3}\right)$ corresponding to the two nodes of $\mathcal{C}_{3}$ form a generalized tacnode, ie either one of them is a cusp or the two branches have the same tangent line; see Zinger [15] for the $n=2$ case.

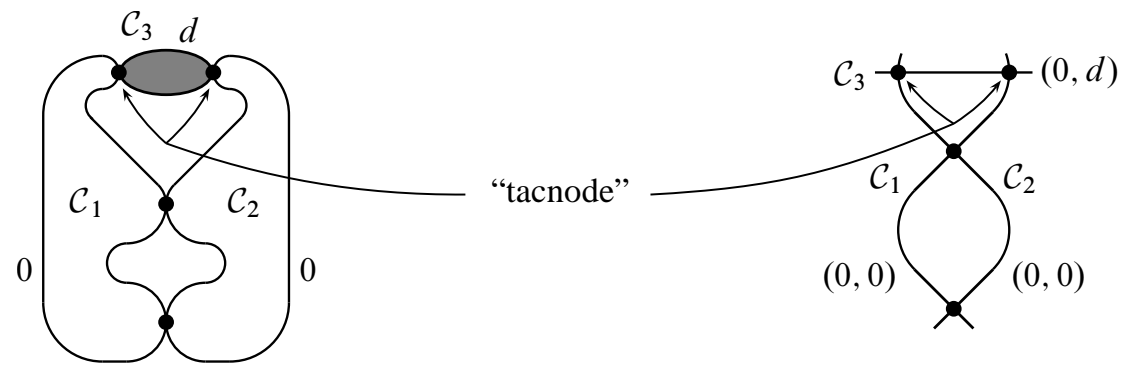

Figure 2: A condition on limits in genus two

The author would like to thank $\mathrm{J}$ Li for suggesting the problem of computing the genus-one GW-invariants of a quintic threefold, which led to the present paper. The author first learned of the arithmetic/geometric-genus compactification terminology in the context of stable maps from G Tian a number of years ago.

\subsection{Compactness theorem}

In this subsection, we describe the subspace $\overline{\mathfrak{M}}_{1, k}^{0}(X, A ; J)$ of $\overline{\mathfrak{M}}_{1, k}(X, A ; J)$; it is a closed subspace by Theorem 1.2. We specify what we mean by a regular almost structure $J$ in Definitions 1.3 and 1.4. If $J$ is genus-one $A$-regular, the moduli space $\overline{\mathfrak{M}}_{1, k}^{0}(X, A ; J)$ has a regular structure, which is described by Theorem 2.3. Since the rather detailed statement of this theorem is notationally involved, we postpone stating it until after we introduce additional notation in Sections 2.1 and 2.2. In this subsection, 
we instead state Corollary 1.5, which describes the two most important consequences of Theorem 2.3.

An element $[\mathcal{C}, u]$ of $\overline{\mathfrak{M}}_{1, k}(X, A ; J)$ is the equivalence class of a pair consisting of a prestable genus-one complex curve $\mathcal{C}$ and a $J$-holomorphic map $u: \mathcal{C} \longrightarrow X$. The prestable curve $\mathcal{C}$ is a union of the principal curve $\mathcal{C}_{P}$, which is either a smooth torus or a circle of spheres, and trees of rational bubble components, which together will be denoted by $\mathcal{C}_{B}$. Let

$$
\mathfrak{M}_{1, k}^{\{0\}}(X, A ; J)=\left\{[\mathcal{C}, u] \in \overline{\mathfrak{M}}_{1, k}(X, A ; J):\left.u\right|_{\mathcal{C}_{P}} \text { is not constant }\right\} \supset \mathfrak{M}_{1, k}^{0}(X, A ; J)
$$

The space $\mathfrak{M}_{1, k}^{\{0\}}(X, A ; J)$ will be a subset of the moduli space $\overline{\mathfrak{M}}_{1, k}^{0}(X, A ; J)$.

Every bubble component $\mathcal{C}_{i} \subset \mathcal{C}_{B}$ is a sphere and has a distinguished singular point, which will be called the attaching node of $\mathcal{C}_{i}$. This is the node of $\mathcal{C}_{i}$ that lies either on $\mathcal{C}_{P}$ or on a bubble $\mathcal{C}_{h}$ that lies between $\mathcal{C}_{i}$ and $\mathcal{C}_{P}$. For example, if $\mathcal{C}$ is as shown in Figure 3, the attaching node of $\mathcal{C}_{h_{3}}$ is the node $\mathcal{C}_{h_{3}}$ shares with the torus. Since $\mathcal{C}_{i}$ is a sphere, we can represent every element of $\overline{\mathfrak{M}}_{1, k}(X, A ; J)$ by a pair $(\mathcal{C}, u)$ such that the attaching node of every bubble component $\mathcal{C}_{i} \subset \mathcal{C}_{B}$ is the south pole, or the point $\infty=(0,0,-1)$, of $S^{2} \subset \mathbb{R}^{3}$. Let $e_{\infty}=(1,0,0)$ be a nonzero tangent vector to $S^{2}$ at the south pole. Then the vector

$$
\left.\mathcal{D}_{i}(\mathcal{C}, u) \equiv d\left\{\left.u\right|_{\mathcal{C}_{i}}\right\}\right|_{\infty} e_{\infty} \in T_{\left.u\right|_{\mathcal{C}_{i}}(\infty)} X
$$

describes the differential of the $J$-holomorphic map $\left.u\right|_{\mathcal{C}_{i}}$ at the attaching node. While this element of $T_{\left.u\right|_{\mathcal{C}_{i}}(\infty)} X$ depends on the choice of a representative for an element of $\overline{\mathfrak{M}}_{1, k}(X, A ; J)$, the linear subspace $\mathbb{C} \cdot \mathcal{D}_{i}(\mathcal{C}, u)$ of $T_{\left.u\right|_{i}}(\infty) X$ is determined by the equivalence class $[\mathcal{C}, u]$. If $\left.u\right|_{\mathcal{C}_{i}}$ is not constant, the branch of the rational $J-$ holomorphic curve $u\left(\mathcal{C}_{i}\right) \subset X$ corresponding to the attaching node of $\mathcal{C}_{i}$ has a cusp if and only if $\mathcal{D}_{i}(\mathcal{C}, u)=0$. If $\mathcal{D}_{i}(\mathcal{C}, u) \neq 0, \mathbb{C} \cdot \mathcal{D}_{i}(\mathcal{C}, u)$ is the line tangent to the branch of $u\left(\mathcal{C}_{i}\right) \subset X$ corresponding to the attaching node of $\mathcal{C}_{i}$.

Suppose $[\mathcal{C}, u] \in \overline{\mathfrak{M}}_{1, k}(X, A ; J)-\mathfrak{M}_{1, k}^{\{0\}}(X, A ; J)$, ie $\left.u\right|_{\mathcal{C}_{P}}$ is constant. In such a case, we will call the bubble sphere $\mathcal{C}_{i} \subset \mathcal{C}_{B}$ first-level $(\mathcal{C}, u)$-effective if $\left.u\right|_{\mathcal{C}_{i}}$ is not constant, but $\left.u\right|_{\mathcal{C}_{h}}$ is constant for every bubble component $\mathcal{C}_{h} \subset \mathcal{C}_{B}$ that lies between $\mathcal{C}_{i}$ and $\mathcal{C}_{P}$. We denote by $\chi(\mathcal{C}, u)$ the set of first-level $(\mathcal{C}, u)$-effective bubbles; see Figure 3 . In this figure, as in Figures 1 and 2, we show the domain $\mathcal{C}$ of the stable map $(\mathcal{C}, u)$ and shade the components of the domain on which the map $u$ is not constant. Note that $u$ maps the attaching nodes of all elements of $\chi(\mathcal{C}, u)$ to the same point in $X$.

Finally, let

$$
H_{2}(X ; \mathbb{Z})^{*}=H_{2}(X ; \mathbb{Z})-\{0\}, \quad \overline{\mathbb{Z}}^{+}=\mathbb{Z}^{+} \sqcup\{0\} .
$$




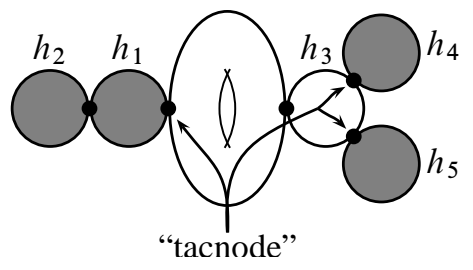

$$
\chi(\mathcal{C}, u)=\left\{h_{1}, h_{4}, h_{5}\right\}
$$

Figure 3: An illustration of Definition 1.1

Definition 1.1 If $(X, \omega, J)$ is a compact almost Kahler manifold, $A \in H_{2}(X ; \mathbb{Z})^{*}$, and $k \in \overline{\mathbb{Z}}^{+}$, the main component of the space $\overline{\mathfrak{M}}_{1, k}(X, A ; J)$ is the subset $\overline{\mathfrak{M}}_{1, k}^{0}(X, A ; J)$ consisting of the elements $[\mathcal{C}, u]$ of $\overline{\mathfrak{M}}_{1, k}(X, A ; J)$ such that

(a) $\left.u\right|_{\mathcal{C}_{P}}$ is not constant, or

(b) $\left.u\right|_{\mathcal{C}_{P}}$ is constant and $\operatorname{dim}_{\mathbb{C}} \operatorname{Span}_{(\mathbb{C}, J)}\left\{\mathcal{D}_{i}(\mathcal{C}, u): i \in \chi(\mathcal{C}, u)\right\}<|\chi(\mathcal{C}, u)|$.

We call a triple $(X, \omega, J)$ an almost Kahler manifold if $\omega$ is a symplectic form on $X$ and $J$ is an almost complex structure on $X$, which is tamed by $\omega$, ie

$$
\omega(v, J v)>0 \quad \forall v \in T X-X .
$$

Definition 1.1 actually involves only the almost complex structure $J$, but one typically considers the moduli spaces $\overline{\mathfrak{M}}_{g, k}(X, A ; J)$ only for $\omega$-tamed almost complex structures $J$, for some symplectic form $\omega$; otherwise, $\overline{\mathfrak{M}}_{g, k}(X, A ; J)$ may not be compact. An element

$$
[\mathcal{C}, u] \in \overline{\mathfrak{M}}_{1, k}(X, A ; J)-\mathfrak{M}_{1, k}^{\{0\}}(X, A ; J)
$$

belongs to $\overline{\mathfrak{M}}_{1, k}^{0}(X, A ; J)$ if and only if the branches of $u(\mathcal{C})$ corresponding to the attaching nodes of the first-level effective bubbles of $[\mathcal{C}, u]$ form a generalized tacnode. In the case of Figure 3, this means that either

(a) for some $i \in\left\{h_{1}, h_{4}, h_{5}\right\}$, the branch of $\left.u\right|_{\mathcal{C}_{i}}$ at the attaching node of $\mathcal{C}_{i}$ has a cusp, or

(b) for all $i \in\left\{h_{1}, h_{4}, h_{5}\right\}$, the branch of $\left.u\right|_{\mathcal{C}_{i}}$ at the attaching node of $\mathcal{C}_{i}$ is smooth, but the dimension of the span of the three lines tangent to these branches is less than three.

This condition is automatically satisfied if $2|\chi(\mathcal{C}, u)|>\operatorname{dim}_{\mathbb{R}} X$. 
Theorem 1.2 If $(X, \omega)$ is a compact symplectic manifold, $\underline{J} \equiv\left(J_{t}\right)_{t \in[0,1]}$ is a $C^{1}-$ continuous family of $\omega$-tamed almost complex structures on $X, A \in H_{2}(X ; \mathbb{Z})^{*}$, and $k \in \overline{\mathbb{Z}}^{+}$, then the moduli space

$$
\overline{\mathfrak{M}}_{1, k}^{0}(X, A ; \underline{J}) \equiv \bigcup_{t \in[0,1]} \overline{\mathfrak{M}}_{1, k}^{0}\left(X, A ; J_{t}\right)
$$

is compact.

If $(X, J)$ is an algebraic variety, the claim of Theorem 1.2, with $J_{t}=J$ constant, is an immediate consequence of well-known results in algebraic geometry. In the case $(X, J)$ is a complex algebraic surface, Lemma 2.4 .1 of Vakil [11] can be used to extend the statement of Theorem 1.2 to all genera.

If $J_{t}=J$ is constant and genus-one $A$-regular in the sense of Definition 1.4 below, Theorem 1.2 follows immediately from the first statement of Theorem 2.3. If $J_{t}$ is genus-one $A$-regular for all $t$, but not necessarily constant, Theorem 1.2 follows from the Gromov Compactness Theorem and Corollary 4.6. In Section 5, we combine the main ingredients of the proof of Theorem 2.3 with the local setting of [6] to obtain Theorem 1.2 with $J_{t}=J$ constant for an arbitrary almost Kahler manifold. The proof for a general family $\underline{J}$ is similar and is described in detail, in an even more general case, in Zinger [19, Section 5].

If $u: \mathcal{C} \longrightarrow X$ is a smooth map from a Riemann surface and $A \in H_{2}(X ; \mathbb{Z})$, we write

$$
u \leq \omega A \quad \text { if } \quad u_{*}[\mathcal{C}]=A \quad \text { or } \quad\left\langle\omega, u_{*}[\mathcal{C}]\right\rangle\langle\langle\omega, A\rangle .
$$

Definition 1.3 Suppose $(X, \omega, J)$ is a compact almost Kahler manifold and $A \in$ $H_{2}(X ; \mathbb{Z})$. The almost complex structure $J$ is genus-zero $A$-regular if for every $J$-holomorphic map $u: \mathbb{P}^{1} \longrightarrow X$ such that $u \leq{ }_{\omega} A$,

(a) the linearization $D_{J, u}$ of the $\bar{\partial}_{J}$-operator at $u$ is surjective;

(b) for all $z \in \mathbb{P}^{1}$, the map $\mathfrak{D}_{J, u}^{z}$ : $\operatorname{ker} D_{J, u} \longrightarrow T_{u(z)} X, \mathfrak{D}_{J, u}^{z}(\xi)=\xi(z)$, is onto.

Definition 1.4 Suppose $(X, \omega, J)$ is a compact almost Kahler manifold and $A \in$ $\mathrm{H}_{2}(X ; \mathbb{Z})$. The almost complex structure $J$ is genus-one $A$-regular if

(a) $J$ is genus-zero $A$-regular;

(b) for every nonconstant $J$-holomorphic map $u: \mathbb{P}^{1} \longrightarrow X$ such that $u \leq_{\omega} A$, (b-i) for all $z \in \mathbb{P}^{1}$ and $v \in T_{z} \mathbb{P}^{1}-\{0\}$, the map

$$
\mathfrak{D}_{J, u}^{z, v}: \operatorname{ker} \mathfrak{D}_{J, u}^{z} \longrightarrow T_{u(z)} X, \quad \mathfrak{D}_{J, u}^{z, v}(\xi)=\nabla_{v} \xi,
$$

is onto; 
(b-ii) for all $z \in \mathbb{P}^{1}$ and $z^{\prime} \in \mathbb{P}^{1}-\{z\}$, the map

$$
\mathfrak{D}_{J, u}^{z, z^{\prime}}: \operatorname{ker} \mathfrak{D}_{J, u}^{z} \longrightarrow T_{u\left(z^{\prime}\right)} X, \quad \mathfrak{D}_{J, u}^{z, z^{\prime}}(\xi)=\xi\left(z^{\prime}\right),
$$

is onto;

(c) for every smooth genus-one Riemann surface $\Sigma$ and every nonconstant $J$ holomorphic map $u: \Sigma \longrightarrow X$ such that $u \leq_{\omega} A$, the linearization $D_{J, u}$ of the $\bar{\partial}_{J}$-operator at $u$ is surjective.

In (b-i) of Definition 1.4, $\nabla_{v} \xi$ denotes the covariant derivative of $\xi$ along $v$, with respect to a connection $\nabla$ in $T X$. Since $\xi(z)=0$, the value of $\nabla_{v} \xi$ is in fact independent of the choice of $\nabla$. If $J$ is an integrable complex structure, the surjectivity statements of (a) and (b) in Definition 1.3 and of (c) in Definition 1.4 can be written as $H^{1}\left(\mathbb{P}^{1} ; u^{*} T X\right)=\{0\}, \quad H^{1}\left(\mathbb{P}^{1} ; u^{*} T X \otimes \mathcal{O}_{\mathbb{P}^{1}}(-1)\right)=\{0\}, \quad H^{1}\left(\Sigma ; u^{*} T X\right)=\{0\}$, respectively. In the integrable case, the two surjectivity statements of (b) in Definition 1.4 are equivalent and can be written as

$$
H^{1}\left(\mathbb{P}^{1} ; u^{*} T X \otimes \mathcal{O}_{\mathbb{P}^{1}}(-2)\right)=\{0\} .
$$

It is well-known that the standard complex structure $J_{0}$ on $\mathbb{P}^{n}$ is genus-one $d \ell$-regular for every $d \in \mathbb{Z}$; see [13, Corollaries 6.3,6.5], for example.

If $J$ is a genus-zero $A$-regular almost complex structure on $X$, the structure of the moduli space $\overline{\mathfrak{M}}_{0, k}(X, A ; J)$ is regular for every $k \in \overline{\mathbb{Z}}^{+}$. In other words, $\overline{\mathfrak{M}}_{0, k}(X, A ; J)$ is stratified by smooth oriented orbifolds of even dimensions and the neighborhood of each stratum has the expected form. One of the results of this paper is that if $J$ is genus-one $A$-regular, the structure of the moduli space $\overline{\mathfrak{M}}_{1, k}^{0}(X, A ; J)$ is regular for every $k \in \overline{\mathbb{Z}}^{+}$; see Theorem 2.3 and Section 4.1. In particular, we have:

Corollary 1.5 (of Theorem 2.3) Suppose $(X, \omega, J)$ is a compact almost Kahler manifold, $A \in H_{2}(X ; \mathbb{Z})^{*}$, and $k \in \mathbb{Z}^{+}$. If $J$ is genus-one $A$-regular, then the closure of $\mathfrak{M}_{1, k}^{0}(X, A ; J)$ in $\overline{\mathfrak{M}}_{1, k}(X, A ; J)$ is $\overline{\mathfrak{M}}_{1, k}^{0}(X, A ; J)$. Furthermore, $\overline{\mathfrak{M}}_{1, k}^{0}(X, A ; J)$ has the general topological structure of a unidimensional algebraic orbivariety ${ }^{1}$ and thus carries a rational fundamental class.

The first statement of Corollary 1.5 follows from the first claim of Theorem 2.3, along with standard gluing arguments such as in McDuff-Salamon [8, Chapter 5]; see also

\footnotetext{
${ }^{1}$ Each point of $\overline{\mathfrak{M}}_{1, k}^{0}(X, A ; J)$ has a neighborhood which is a quotient of an affine algebraic variety of complex dimension $\operatorname{dim}_{1, k}(X, A) / 2$ by a finite group.
} 
Section 4.1. The middle statement of Corollary 1.5 summarizes Theorem 2.3, while the last one is obtained at the end of Section 2.3.

We will also show that the genus-zero and genus-one regularity properties are wellbehaved under small perturbations:

Theorem 1.6 Suppose $(X, \omega, J)$ is a compact almost Kahler manifold and $A \in$ $H_{2}(X ; \mathbb{Z})^{*}$. If $g=0,1$ and the almost complex structure $J$ is genus- $g A$-regular, then there exists $\delta_{J}(A) \in \mathbb{R}^{+}$with the property that if $\widetilde{J}$ is an almost complex structure on $X$ such that $\|\widetilde{J}-J\|_{C^{1}} \leq \delta_{J}(A)$, then $\widetilde{J}$ is genus- $g$-regular. Furthermore, if $J$ is genus-one $A$-regular, $k \in \overline{\mathbb{Z}}^{+}$, and $\underline{J}=\left(J_{t}\right)_{t \in[0,1]}$ is a continuous family of almost complex structures on $X$ such that $J_{0}=J$ and $\left\|J_{t}-J\right\|_{C^{1}} \leq \delta_{J}(A)$ for all $t \in[0,1]$, then the moduli space $\overline{\mathfrak{M}}_{1, k}^{0}(X, A ; \underline{J})$ has the general topological structure of a unidimensional algebraic orbivariety with boundary ${ }^{2}$ and

$$
\partial \overline{\mathfrak{M}}_{1, k}^{0}(X, A ; \underline{J})=\overline{\mathfrak{M}}_{1, k}^{0}\left(X, A ; J_{1}\right)-\overline{\mathfrak{M}}_{1, k}^{0}\left(X, A ; J_{0}\right) .
$$

The norms $\|\tilde{J}-J\|_{C^{1}}$ and $\left\|J_{t}-J\right\|_{C^{1}}$ are computed using a fixed connection in the vector bundle $T X$, eg the Levi-Civita connection of the metric on $X$ determined by $(\omega, J)$. The regularity claims of Theorem 1.6 follow from the compactness of the moduli spaces $\overline{\mathfrak{M}}_{g, k}(X, A ; J)$ and Corollaries 3.2, 3.6, 3.7, 4.2 and 4.5. The final claim of Theorem 1.6 follows from a family version of Theorem 2.3. It can in fact be used to show that under the assumptions of Theorem 1.6

$$
\overline{\mathfrak{M}}_{1, k}^{0}(X, A ; \underline{J}) \approx[0,1] \times \overline{\mathfrak{M}}_{1, k}^{0}\left(X, A ; J_{0}\right) .
$$

The conclusion, as stated, can be obtained with weaker regularity assumptions on $\underline{J}$.

The key ingredients in the proofs of Theorems 1.6 and 2.3 are the gluing constructions of Zinger [16], adapted to the present situation, and the power series expansions of Theorem 2.8 and Section 4.1 in Zinger [13], applied via a technical result of Floer, Hofer and Salamon [3]. The power series of [13] give estimates on the behavior of derivatives of holomorphic maps under gluing and on the obstructions to smoothing holomorphic maps from singular domains. A technical result of [3] shows that locally a $J$-holomorphic map is very close to a holomorphic one. Ionel [5] essentially shows that the above obstructions do not vanish on the complement of $\overline{\mathfrak{M}}_{1, k}^{0}\left(\mathbb{P}^{n}, d\right)$ in $\overline{\mathfrak{M}}_{1, k}\left(\mathbb{P}^{n}, d\right)$; this is the main portion of Theorem 1.2 for $(X, J)=\left(\mathbb{P}^{n}, J_{0}\right)$ and thus for all algebraic varieties. This theorem can be viewed as describing limits in $\overline{\mathfrak{M}}_{1, k}(X, A ; J)$; in comparison, Theorem 2.3 can be viewed as describing limiting behavior.

\footnotetext{
${ }^{2}$ Each point of $\overline{\mathfrak{M}}_{1, k}^{0}(X, A ; \underline{J})$ has a neighborhood which is a quotient of a fibration over $(0,1)$ or $[0,1)$ of affine algebraic varieties of complex dimension $\operatorname{dim}_{1, k}(X, A) / 2$ by a finite group.
} 


\subsection{Some geometric implications}

Theorem 1.2 implies that under certain assumptions on $A$ and $J$ the number of genusone degree- $A J$-holomorphic curves that pass through a collection of cycles in $X$ of the appropriate total codimension is finite. Furthermore, each such curve is isolated to first order, as explained below. Throughout this subsection, we assume that the dimension of $X$ is $2 n \geq 4$.

A simple $J$-holomorphic map into $X$ is a $J$-holomorphic map $u$ : $\Sigma \longrightarrow X$ such that $u$ is one-to-one outside of finitely many points of $\Sigma$ and the irreducible components of $\Sigma$ on which $u$ is constant. A genus $-g$ degree $-A J$-holomorphic curve $\mathcal{C}$ is the image $u(\Sigma)$ of an element $[\Sigma, u]$ of $\overline{\mathfrak{M}}_{g, k}(X, A ; J)$ such that $u$ is simple and the total genus of the components on which $u$ is not constant is $g$. Let $\mathcal{M}_{g}(X, A ; J)$ be the space of all genus- $g$ degree- $A J$-holomorphic curves in $X$. The expected dimension of this space is $\operatorname{dim}_{g, 0}(X, A)$.

A $J$-holomorphic curve $\mathcal{C} \subset X$ will be called regular if the operator $D_{J, u}$ is surjective for a (or equivalently, every) stable-map parametrization $u: \Sigma \longrightarrow \mathcal{C}$ of $\mathcal{C}$ as above. We will call a regular curve $\mathcal{C} \subset X$ essentially embedded if $\mathcal{C}$ is an irreducible curve that has no singularities if $n \geq 3$ and its only singularities are simple nodes if $n=2$. In other words, if $u: \Sigma \longrightarrow \mathcal{C}$ is a parametrization of $\mathcal{C}$ with $k=0$, then $\Sigma$ is a smooth Riemann surface of genus $g$. Furthermore, if $n \geq 3, u$ is an embedding. If $n=2$, then

$$
\operatorname{dim}_{\mathbb{C}} \operatorname{Span}_{\mathbb{C}}\left\{\left.\operatorname{Im} d u\right|_{z}: z \in u^{-1}(q)\right\}=\left|u^{-1}(q)\right| \quad \forall q \in X .
$$

In particular, $u$ is an immersion.

Let $\mu=\left(\mu_{1}, \ldots, \mu_{k}\right)$ be a $k$-tuple of cycles in $X$ of total (real) codimension $\operatorname{dim}_{g, 0}(X, A)+2 k$, ie

$$
\operatorname{codim} \mu \equiv \sum_{l=1}^{l=k} \operatorname{codim} \mu_{l}=\operatorname{dim}_{g, 0}(X, A)+2 k=\operatorname{dim}_{g, k}(X, A) .
$$

We denote by $\mathcal{M}_{g}(X, A ; J, \mu)$ the set of genus- $g$ degree- $A J$-holomorphic curves that pass through every cycle $\mu_{1}, \ldots, \mu_{k}$, ie,

$$
\begin{aligned}
& \mathcal{M}_{g}(X, A ; J, \mu)=\left\{\mathcal{C} \in \mathcal{M}_{g}(X, A ; J): \mathcal{C} \cap \mu_{l} \neq \varnothing \forall l \in[k]\right\} \text {, } \\
& \text { where } \quad[k]=\{1, \ldots, k\} .
\end{aligned}
$$

We will call an element $\mathcal{C}$ of $\mathcal{M}_{g}(X, A ; J, \mu)$ isolated to first order if for every parametrization

$$
u: \Sigma \longrightarrow \mathcal{C} \subset X
$$


of $\mathcal{C}$, where $\Sigma$ is a curve with $k$ marked points $y_{1}, \ldots, y_{k}$ such that $u\left(y_{l}\right) \in \mu_{l}$ for all $l \in[k]$,

$\left\{\xi \in \operatorname{ker} D_{J, u}: \xi\left(y_{l}\right) \in T_{u\left(y_{l}\right)} \mu_{l}+\left.\operatorname{Im} d u\right|_{y_{l}} \forall l \in[k]\right\} \subset \Gamma(\Sigma ; \operatorname{Im} d u) \subset \Gamma\left(\Sigma ; u^{*} T X\right)$.

If $\mathcal{M}_{g}(X, A ; J)$ is a smooth manifold with the expected tangent bundle and the constraints $\mu_{1}, \ldots, \mu_{k}$ are in general position, then $\mathcal{M}_{g}(X, A ; J, \mu)$ is a discreet set consisting of elements isolated to first order. Below we describe some circumstances under which this set is also finite.

We recall that $A \in H_{2}(X ; \mathbb{Z})$ is called spherical if

$$
A=f_{*}\left[S^{2}\right] \in H_{2}(X ; \mathbb{Z})
$$

for some smooth map $f: S^{2} \longrightarrow X$. A symplectic manifold $(X, \omega)$ is weakly monotone if for every spherical homology class $A$ such that $\omega(A)>0$, either

$$
\left\langle c_{1}(T X), A\right\rangle \geq 0 \quad \text { or } \quad\left\langle c_{1}(T X), A\right\rangle \leq 2-n,
$$

where $2 n=\operatorname{dim} X$, as before. In particular, all symplectic manifolds of (real) dimensions 2, 4, and 6 are weakly monotone. So are all complex projective spaces, which are in fact monotone; see McDuff-Salamon [8, Chapter 5] for a definition.

Finally, if $(X, \omega)$ is a symplectic manifold, we denote by $\mathcal{J}(X, \omega)$ the space of all almost complex structures on $X$ tamed by $\omega$, endowed with the $C^{1}$-topology.

Proposition 1.7 Suppose $(X, \omega, J)$ is a compact almost Kahler manifold, $A \in$ $H_{2}(X ; \mathbb{Z})^{*}, g=0,1$, and $J$ is genus-g $A$-regular. If $\mu$ is a $k$-tuple of cycles in $X$ of total codimension $\operatorname{dim}_{g, k}(X, A)$ in general position, then $\mathcal{M}_{g}(X, A ; J, \mu)$ is a finite set and every element in $\mathcal{M}_{g}(X, A ; J, \mu)$ is irreducible, regular, and isolated to first order.

Proposition 1.8 If $(X, \omega)$ is a compact weakly monotone symplectic manifold and $A \in H_{2}(X ; \mathbb{Z})^{*}$, there exists a dense open subset $\mathcal{J}_{\text {reg }}(X, \omega ; A)$ of $\mathcal{J}(X, \omega)$ with the following properties. If $J \in \mathcal{J}_{\text {reg }}(X, \omega ; A), g=0,1$, and $\mu$ is a $k$-tuple of cycles in $X$ of total codimension $\operatorname{dim}_{g, k}(X, A)$ in general position, then every element in $\mathcal{M}_{g}(X, A ; J, \mu)$ is essentially embedded and isolated to first order. If in addition $\left\langle c_{1}(T X), A\right\rangle \neq 0$, then $\mathcal{M}_{g}(X, A ; J, \mu)$ is a finite set and its signed cardinality is independent of the choice of $J \in \mathcal{J}_{\text {reg }}(X, \omega ; A)$.

Most of the genus-zero statements of these two propositions are well-known; see McDuff-Salamon [8, Chapters 5-7], for example. The signed cardinality of the set $\mathcal{M}_{0}(X, A ; J, \mu)$ is the corresponding Gromov-Witten invariant, $\mathrm{GW}_{0, k}(A ; \mu)$, 
of $(X, \omega)$. The remaining statements are obtained from minor extensions of some results in [8], along with Theorem 1.2 in the genus-one case. For each $l \in[k]$, let

$$
\mathrm{ev}_{l}: \overline{\mathfrak{M}}_{g, k}(X, A ; J) \longrightarrow X, \quad\left[\Sigma, y_{1}, \ldots, y_{k} ; u\right] \longrightarrow u\left(y_{l}\right)
$$

be the evaluation map for the $l$-th marked point.

Suppose $(X, \omega, J)$ and $A$ are as in the statement of Proposition 1.7 and $J$ is genus-one $A$-regular. By (c) of Definition 1.4, the moduli space $\mathfrak{M}_{1, k}^{0}(X, A ; J)$ is a smooth orbifold with the expected tangent bundle. Thus, if $\mu$ is a tuple of constraints as in the $g=1$ case of Proposition 1.7, then

$$
\mathfrak{M}_{1, k}^{0}(X, A ; J, \mu) \equiv\left\{b \in \mathfrak{M}_{1, k}^{0}(X, A ; J): \operatorname{ev}_{l}(b) \in \mu_{l} \forall l \in[k]\right\}
$$

is a zero-dimensional oriented submanifold. If $\left\{b_{r}\right\}$ is a sequence of distinct elements in $\mathfrak{M}_{1, k}^{0}(X, A ; J, \mu)$, by Theorem 1.2 a subsequence of $\left\{b_{r}\right\}$ must converge to an element

$$
\begin{aligned}
b \in \overline{\mathfrak{M}}_{1, k}^{0}(X, A ; J, \mu) \equiv & \left\{b \in \overline{\mathfrak{M}}_{1, k}^{0}(X, A ; J): \operatorname{ev}_{l}(b) \in \mu_{l} \forall l \in[k]\right\} \\
& \subset \overline{\mathfrak{M}}_{1, k}(X, A ; J) .
\end{aligned}
$$

Since all elements of $\mathfrak{M}_{1, k}^{0}(X, A ; J, \mu)$ are isolated,

$$
b \in \overline{\mathfrak{M}}_{1, k}^{0}(X, A ; J, \mu)-\mathfrak{M}_{1, k}^{0}(X, A ; J) .
$$

On the other hand, by the regularity assumptions of Definition 1.4,

$$
\partial \overline{\mathfrak{M}}_{1, k}^{0}(X, A ; J) \equiv \overline{\mathfrak{M}}_{1, k}^{0}(X, A ; J)-\mathfrak{M}_{1, k}^{0}(X, A ; J)
$$

is a union of strata of dimensions smaller than $\operatorname{dim}_{1, k}(X, A)$. Thus, if $\mu$ is a tuple of cycles of total codimension $\operatorname{dim}_{1, k}(X, A)$ in general position, then

$$
\overline{\mathfrak{M}}_{1, k}^{0}(X, A ; J, \mu)-\mathfrak{M}_{1, k}^{0}(X, A ; J)=\varnothing .
$$

It follows that $\mathfrak{M}_{1, k}^{0}(X, A ; J, \mu)$ is a finite set, and so is its subset $\mathcal{M}_{1}(X, A ; J, \mu)$.

We next move to Proposition 1.8. For any $B \in H_{2}(X ; \mathbb{Z})^{*}$, almost complex structure $J$ on $X$, and $g \in \mathbb{Z}^{+}$, let

$$
\begin{aligned}
& \mathfrak{M}_{g, k}^{\text {simp }}(X, B ; J) \subset \mathfrak{M}_{g, k}^{0}(X, B ; J), \\
& \mathfrak{M}_{g, k}^{\{\operatorname{simp}\}}(X, B ; J) \subset \mathfrak{M}_{g, k}^{\{0\}}(X, B ; J), \\
& \overline{\mathfrak{M}}_{g, k}^{\text {simp }}(X, B ; J) \subset \overline{\mathfrak{M}}_{g, k}(X, B ; J)
\end{aligned}
$$


denote the subspaces of simple maps. By [8, Chapter 3], for a generic choice of $J$, $D_{J, u}$ is surjective for every element $[\Sigma, u]$ of $\mathfrak{M}_{g, k}^{\text {simp }}(X, A ; J)$. Thus, as before,

$$
\mathcal{M}_{1}(X, A ; J, \mu) \approx \mathfrak{M}_{1, k}^{\text {simp }}(X, A ; J, \mu) \equiv \mathfrak{M}_{1, k}^{0}(X, A ; J, \mu) \cap \mathfrak{M}_{1, k}^{\text {simp }}(X, A ; J)
$$

is a zero-dimensional oriented manifold, if $\mu$ is as in Proposition 1.8. On the other hand, by the same argument as in [8, Chapter 6], the evaluation map

$$
\mathrm{ev}_{k+1} \times \mathrm{ev}_{k+2}: \mathfrak{M}_{1, k+2}^{\mathrm{simp}}(X, A ; J) \longrightarrow X \times X
$$

is transverse to the diagonal, for a generic choice of $J$. Let

$$
L_{k+1} \longrightarrow \mathfrak{M}_{1, k+1}^{\text {simp }}(X, A ; J)
$$

be the universal tangent line bundle for the last marked point, ie

$$
\left.L_{k+1}\right|_{[\Sigma, u]}=T_{y_{k+1}} \Sigma \quad \forall[\Sigma, u] \in \mathfrak{M}_{1, k+1}^{\text {simp }}(X, A ; J) .
$$

By a small modification of the proof of Lemma 6.1.2 in [8], the bundle section

$$
\left.d u\right|_{y_{k+1}}: \mathfrak{M}_{1, k+1}^{\mathrm{simp}}(X, A ; J) \longrightarrow L_{k+1}^{*} \otimes \operatorname{ev}_{k+1}^{*} T X,\left.\quad[\Sigma, u] \longrightarrow d u\right|_{y_{k+1}},
$$

is transverse to the zero set, for a generic choice of $J$. The key part of this modification is to view the relevant first-order equation as an elliptic operator acting on the space of smooth sections of the vector bundle $n \mathcal{O}_{\mathbb{P}^{1}}(1)$ over $S^{2}$. The last two transversality properties imply that for a generic element $[\Sigma, u]$ of $\mathfrak{M}_{1, k}^{\{\text {simp }\}}(X, A ; J)$ its image $u(\Sigma)$ is essentially embedded. This concludes the proof of the first statement of Proposition 1.8 .

By [8, Chapters 3,6], for a generic choice of $J, D_{J, u}$ is surjective for every element $[\Sigma, u]$ of $\mathfrak{M}_{g, k}^{\{s i m p\}}(X, B ; J)$. In particular, $\mathfrak{M}_{g, k}^{\{\operatorname{simp}\}}(X, B ; J)$ is a finite union of smooth orbifolds of the expected dimension. Thus, if $\mu$ is a tuple of constraints as in the statement of Proposition 1.8,

$$
\left\{b \in \mathfrak{M}_{1, k}^{\{\operatorname{simp}\}}(X, A ; J): \operatorname{ev}_{l}(b) \in \mu_{l} \forall l \in[k]\right\}-\mathfrak{M}_{1, k}^{0}(X, A ; J)=\varnothing .
$$

Furthermore, if $\left\langle c_{1}(T X), A\right\rangle \neq 0$, every element of

$$
\overline{\mathfrak{M}}_{1, k}(X, A ; J, \mu) \equiv\left\{b \in \overline{\mathfrak{M}}_{1, k}(X, A ; J): \operatorname{ev}_{l}(b) \in \mu_{l} \forall l \in[k]\right\}
$$

is simple. This can be seen by considering the dimension of the image of the multiply covered elements of $\overline{\mathfrak{M}}_{1, k}(X, A ; J)$ under the evaluation map ev $1 \times \cdots \times \mathrm{ev}_{k}$. This is done by passing to moduli spaces of maps consisting of simple elements; see [8, Chapter 5]. The argument requires two separate dimension counts for multiply covered maps: one for the elements in $\mathfrak{M}_{1, k}^{\{0\}}(X, A ; J)$ and the other for those in its complement in $\overline{\mathfrak{M}}_{1, k}(X, A ; J)$. In addition to the assumption $\left\langle c_{1}(T X), A\right\rangle \neq 0$, the 
weakly monotone condition on $(X, \omega)$ enters directly into both dimension computations. Finally, by the same modification of the proof of Lemma 6.1.2 in [8] as described above, but applied to tuples of genus-zero maps instead of genus-one maps,

$$
\overline{\mathfrak{M}}_{1, k}^{\text {simp }}(X, A ; J) \cap\left(\overline{\mathfrak{M}}_{1, k}^{0}(X, A ; J)-\mathfrak{M}_{1, k}^{\{0\}}(X, A ; J)\right)
$$

is a finite union of smooth orbifolds of dimensions less than $\operatorname{dim}_{1, k}(X, A)$. We conclude that

$$
\mathcal{M}_{1}(X, A ; J, \mu) \approx \mathfrak{M}_{1, k}^{\text {simp }}(X, A ; J, \mu)=\overline{\mathfrak{M}}_{1, k}^{0}(X, A ; J, \mu)
$$

is a compact zero-dimensional manifold. By a cobordism argument as in [8, Chapter 7], the signed cardinality of $\mathcal{M}_{1}(X, A ; J, \mu)$ is independent of a generic choice of $J$.

The signed cardinality $\mathrm{GW}_{1, k}^{0}(A ; \mu)$ of the set $\mathcal{M}_{1, k}(X, A ; J, \mu)$ is an integer-valued invariant of the symplectic manifold $(X, \omega)$. The difference between this invariant for an arbitrary symplectic manifold (when the invariant may not be an integer) and the standard genus-one GW-invariant is described by [19, Proposition 3.1] and explicitly given by [21, Theorems 1A,1B].

\section{Preliminaries}

\subsection{Notation: Genus-zero maps}

We now describe our notation for bubble maps from genus-zero Riemann surfaces, for the spaces of such bubble maps that form the standard stratifications of the moduli spaces of stable maps, and for important vector bundles over them. In general, the moduli spaces of stable maps can stratified by the dual graph. However, in the present situation, it is more convenient to make use of linearly ordered sets:

Definition 2.1 (1) A finite nonempty partially ordered set $I$ is a linearly ordered set if for all $i_{1}, i_{2}, h \in I$ such that $i_{1}, i_{2}<h$, either $i_{1} \leq i_{2}$ or $i_{2} \leq i_{1}$.

(2) A linearly ordered set $I$ is a rooted tree if $I$ has a unique minimal element, ie there exists $\hat{0} \in I$ such that $\hat{0} \leq i$ for all $i \in I$.

We use rooted trees to stratify the moduli space $\overline{\mathfrak{M}}_{0,\{0\} \sqcup M}(X, A ; J)$ of genus-zero stable holomorphic maps with marked points indexed by the set $\{0\} \sqcup M$, where $M$ is a finite set.

If $I$ is a linearly ordered set, let $\widehat{I}$ be the subset of the nonminimal elements of $I$. For every $h \in \hat{I}$, denote by $\iota_{h} \in I$ the largest element of $I$ which is smaller than $h$, ie $\iota_{h}=\max \{i \in I: i<h\}$. 
We identify $\mathbb{C}$ with $S^{2}-\{\infty\}$ via the stereographic projection mapping the origin in $\mathbb{C}$ to the north pole, or the point $(0,0,1)$, in $S^{2}$. A genus-zero $X$-valued bubble map with $M$-marked points is a tuple

$$
b=(M, I ; x,(j, y), u),
$$

where $I$ is a rooted tree, and

(2-1) $x: \hat{I} \longrightarrow \mathbb{C}=S^{2}-\{\infty\}, j: M \longrightarrow I, \quad y: M \longrightarrow \mathbb{C}, u: I \longrightarrow C^{\infty}\left(S^{2} ; X\right)$

are maps such that $u_{h}(\infty)=u_{\iota_{h}}\left(x_{h}\right)$ for all $h \in \hat{I}$. We associate such a tuple with Riemann surface

$$
\Sigma_{b}=\left(\bigsqcup_{i \in I} \Sigma_{b, i}\right) / \sim, \quad \text { where } \Sigma_{b, i}=\{i\} \times S^{2},(h, \infty) \sim\left(\iota_{h}, x_{h}\right) \forall h \in \hat{I}
$$

with marked points

$$
y_{l}(b) \equiv\left(j_{l}, y_{l}\right) \in \Sigma_{b, j_{l}} \quad \text { and } \quad y_{0}(b) \equiv(\widehat{0}, \infty) \in \Sigma_{b, \hat{0}}
$$

and with the continuous map $u_{b}: \Sigma_{b} \longrightarrow X$, given by $\left.u_{b}\right|_{\Sigma_{b, i}}=u_{i}$ for all $i \in I$. The general structure of bubble maps is described by tuples $\mathcal{T}=(M, I ; j, \underline{A})$, where

$$
A_{i}=\left\{\left.u_{b}\right|_{\Sigma_{b, i}}\right\}_{*}\left[S^{2}\right] \quad \forall i \in I .
$$

We call such tuples bubble types. Denote by $\mathcal{U}_{\mathcal{T}}(X ; J)$ the subset of $\overline{\mathfrak{M}}_{0,\{0\} \sqcup M}(X, A ; J)$ consisting of stable maps $[\mathcal{C}, u]$ such that

$$
[\mathcal{C} ; u]=\left[\left(\Sigma_{b},(\widehat{0}, \infty),\left(j_{l}, y_{l}\right)_{l \in M}\right) ; u_{b}\right],
$$

for some bubble map $b$ of type $\mathcal{T}$ as above, where $\hat{0}$ is the minimal element of $I$; see Zinger [16, Section 2] for details. For $l \in\{0\} \sqcup M$, let

$$
\mathrm{ev}_{l}: \mathcal{U}_{\mathcal{T}}(X ; J) \longrightarrow X
$$

be the evaluation map corresponding to the marked point $y_{l}$.

We denote the bundle of gluing parameters, or of smoothings at the nodes, over $\mathcal{U}_{\mathcal{T}}(X ; J)$ by $\mathcal{F} \mathcal{T}$. This orbi-bundle has the form

$$
\mathcal{F} \mathcal{T}=\left(\bigoplus_{h \in \hat{I}} L_{h, 0} \otimes L_{h, 1}\right) / \operatorname{Aut}(\mathcal{T}),
$$

for certain line orbi-bundles $L_{h, 0}$ and $L_{h, 1}$. These line bundles ${ }^{3}$ are the line bundles associated to certain $S^{1}$-principal bundles. More precisely, there exists a subspace

${ }^{3}$ also known as the universal tangent line bundles at the node, but this is not essential here 
$\mathcal{U}_{\mathcal{T}}^{(0)}(X ; J)$ of the space $\mathcal{H}_{\mathcal{T}}(X ; J)$ of $J$-holomorphic maps into $X$ of type $\mathcal{T}$, not of equivalence classes of such maps, such that

$$
\mathcal{U}_{\mathcal{T}}(X ; J)=\mathcal{U}_{\mathcal{T}}^{(0)}(X ; J) / \operatorname{Aut}(\mathcal{T}) \propto\left(S^{1}\right)^{I}
$$

The line bundles $L_{h, 0}$ and $L_{h, 1}$ arise from this quotient; see Section 2.5 in [16]. In particular,

$$
\mathcal{F} \mathcal{T}=\widetilde{\mathcal{F}} \mathcal{T} / \operatorname{Aut}(\mathcal{T}) \propto\left(S^{1}\right)^{I}, \quad \text { where } \quad \widetilde{\mathcal{F}} \mathcal{T}=\mathcal{U}_{\mathcal{T}}^{(0)}(X ; J) \times \mathbb{C}^{\widehat{I}} \longrightarrow \mathcal{U}_{\mathcal{T}}^{(0)}(X ; J)
$$

We denote by $\mathcal{F} \mathcal{T}^{\varnothing}$ and $\widetilde{\mathcal{F}} \mathcal{T}^{\varnothing}$ the subsets of $\mathcal{F} \mathcal{T}$ and $\widetilde{\mathcal{F}} \mathcal{T}$, respectively, consisting of the elements with all components nonzero.

The subset $\mathcal{U}_{\mathcal{T}}^{(0)}(X ; J)$ of $\mathcal{H}_{\mathcal{T}}(X ; J)$ is described by the conditions (B1) and (B2) in Section 2.5 of [16]. It is the preimage of the point $(0,1 / 2)^{I}$ in $(\mathbb{C} \times \mathbb{R})^{I}$ under the continuous map

$$
\Psi_{\mathcal{T}} \equiv\left(\Psi_{\mathcal{T}, i}\right)_{i \in I}: \mathcal{H}_{\mathcal{T}}(X ; J) \longrightarrow(\mathbb{C} \times \mathbb{R})^{I}
$$

defined in the proof of Proposition 3.3 in [16]. The statements of the conditions (B1) and (B2) and the definition of the map $\Psi_{\mathcal{T}}$ require a choice of a $J$-compatible metric $g_{X}$. It can be assumed that

$$
\int_{\mathbb{P}^{1}}|d u|_{g_{X}}^{2} \geq 1
$$

for every nonconstant $J$-holomorphic maps $u$ : $\mathbb{P}^{1} \longrightarrow X$. Such a metric $g_{X}$ will be fixed once and for all. If the almost complex structure $J$ is genus-zero $A$-regular, where $A=\sum_{i \in I} A_{i}$, the space $\mathcal{H}_{\mathcal{T}}(X ; J)$ is a smooth manifold of the expected dimension; see Chapter 3 in [8]. In such a case, the map $\Psi_{\mathcal{T}}$ is smooth and transversal to every point $\left(0, r_{i}\right)_{i \in I}$ such that $\left|r_{i}-\frac{1}{2}\right| \leq \frac{1}{4}$ for all $i \in I$; see the proof of Proposition 3.3 in [16]. Let

$$
\begin{aligned}
\chi(\mathcal{T}) & =\left\{i \in I: A_{i} \neq 0 ; A_{h}=0 \forall h<i\right\}, \\
\tilde{\mathcal{U}}_{\mathcal{T}}^{(0)}(X ; J) & =\Psi_{\mathcal{T}}^{-1}\left(\left\{\left(0, r_{i}\right)_{i \in I} \in(\mathbb{C} \times \mathbb{R})^{I}:\right.\right. \\
\left.r_{i}=\frac{1}{2} \forall i \in I-\chi(\mathcal{T}), r_{i} \in\left(\frac{1}{4}, \frac{3}{4}\right) \forall i \in \chi(\mathcal{T})\right\}, & \\
\widetilde{\mathcal{F}} \mathcal{T} & =\widetilde{\mathcal{U}}_{\mathcal{T}}^{(0)}(X ; J) \times \mathbb{C}^{\widehat{I}} \longrightarrow \widetilde{\mathcal{U}}_{\mathcal{T}}^{(0)}(X ; J) .
\end{aligned}
$$

As before, we denote by $\widetilde{\mathcal{F T}}^{\varnothing}$ the subset of $\widetilde{\mathcal{F} \mathcal{T}}$ consisting of the elements with all components nonzero. 


\subsection{Notation: Genus-one maps}

We next set up analogous notation for maps from genus-one Riemann surfaces. In this case, we also need to specify the structure of the principal component. Thus, we index the strata of the moduli space $\overline{\mathfrak{M}}_{1, M}(X, A ; J)$ by enhanced linearly ordered sets:

Definition 2.2 An enhanced linearly ordered set is a pair $(I, \boldsymbol{\aleph})$, where $I$ is a linearly ordered set, $\boldsymbol{\aleph}$ is a subset of $I_{0} \times I_{0}$, and $I_{0}$ is the subset of minimal elements of $I$, such that if $\left|I_{0}\right|>1$,

$$
\aleph=\left\{\left(i_{1}, i_{2}\right),\left(i_{2}, i_{3}\right), \ldots,\left(i_{n-1}, i_{n}\right),\left(i_{n}, i_{1}\right)\right\}
$$

for some bijection $i:\{1, \ldots, n\} \longrightarrow I_{0}$.

An enhanced linearly ordered set can be represented by an oriented connected graph. In Figure 4, the dots denote the elements of $I$. The arrows outside the loop, if there are any, specify the partial ordering of the linearly ordered set $I$. In fact, every directed edge outside of the loop connects a nonminimal element $h$ of $I$ with $\iota_{h}$. Inside of the loop, there is a directed edge from $i_{1}$ to $i_{2}$ if and only if $\left(i_{1}, i_{2}\right) \in \aleph$.
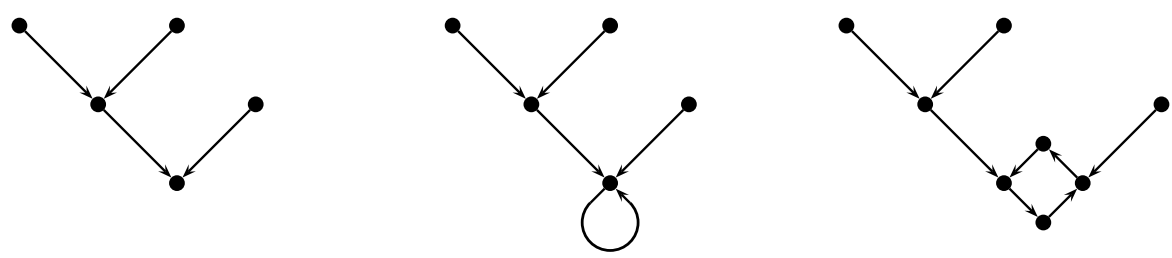

Figure 4: Some enhanced linearly ordered sets

The subset $\aleph$ of $I_{0} \times I_{0}$ will be used to describe the structure of the principal curve of the domain of stable maps in a stratum of the moduli space $\overline{\mathfrak{M}}_{1, M}(X, A ; J)$. If $\boldsymbol{\aleph}=\varnothing$, and thus $\left|I_{0}\right|=1$, the corresponding principal curve $\Sigma_{\aleph}$ is a smooth torus, with some complex structure. If $\aleph \neq \varnothing$, the principal components form a circle of spheres:

$$
\Sigma_{\aleph}=\left(\bigsqcup_{i \in I_{0}}\{i\} \times S^{2}\right) / \sim, \quad \text { where } \quad\left(i_{1}, \infty\right) \sim\left(i_{2}, 0\right) \text { if }\left(i_{1}, i_{2}\right) \in \aleph .
$$

A genus-one $X$-valued bubble map with $M$-marked points is a tuple

$$
b=(M, I, \aleph ; S, x,(j, y), u),
$$

where $S$ is a smooth Riemann surface of genus one if $\boldsymbol{\aleph}=\varnothing$ and the circle of spheres $\Sigma_{\aleph}$ otherwise. The objects $x, j, y, u$, and $\left(\Sigma_{b}, u_{b}\right)$ are as in (2-1) and (2-2), except 
the sphere $\Sigma_{b, \hat{0}}$ is replaced by the genus-one curve $\Sigma_{b, \aleph} \equiv S$. Furthermore, if $\aleph=\varnothing$, and thus $I_{0} \stackrel{b, \hat{0}}{=}\{\hat{0}\}$ is a single-element set, $u_{\hat{0}} \in C^{\infty}(S ; X)$ and $y_{l} \in S$ if $j_{l}=\widehat{0}$. In the genus-one case, the general structure of bubble maps is encoded by the tuples of the form $\mathcal{T}=(M, I, \boldsymbol{\aleph} ; j, \underline{A})$. Similarly to the genus-zero case, we denote by $\mathcal{U}_{\mathcal{T}}(X ; J)$ the subset of $\overline{\mathfrak{M}}_{1, M}(X, A ; J)$ consisting of stable maps $[\mathcal{C}, u]$ such that

$$
[\mathcal{C} ; u]=\left[\left(\Sigma_{b},\left(j_{l}, y_{l}\right)_{l \in M}\right) ; u_{b}\right]
$$

for some bubble map $b$ of type $\mathcal{T}$ as above.

If $\mathcal{T}=(M, I, \boldsymbol{\aleph} ; j, \underline{A})$ is a bubble type as above, let

$$
\begin{gathered}
I_{1}=\left\{h \in \widehat{I}: \iota_{h} \in I_{0}\right\}, \quad M_{0}=\left\{l \in M: j_{l} \in I_{0}\right\}, \\
\mathcal{T}_{0}=\left(M_{0} \sqcup I_{1}, I_{0}, \aleph ;\left.\left.j\right|_{M_{0}} \sqcup \iota\right|_{I_{1}},\left.\underline{A}\right|_{I_{0}}\right),
\end{gathered}
$$

where $I_{0}$ is the subset of minimal elements of $I$. For each $h \in I_{1}$, we put

$$
I_{h}=\{i \in I: h \leq i\}, \quad M_{h}=\left\{l \in M: j_{l} \in I_{h}\right\}, \quad \mathcal{T}_{h}=\left(M_{h}, I_{h} ;\left.j\right|_{M_{h}},\left.\underline{A}\right|_{I_{h}}\right) .
$$

We have a natural isomorphism

$$
\begin{aligned}
\mathcal{U}_{\mathcal{T}}(X ; J) \approx\left(\left\{\left(b_{0},\left(b_{h}\right)_{h \in I_{1}}\right) \in \mathcal{U}_{\mathcal{T}_{0}}(X ; J) \times \prod_{h \in I_{1}} \mathcal{U}_{\mathcal{T}_{h}}(X ; J):\right.\right. \\
\left.\left.\operatorname{ev}_{0}\left(b_{h}\right)=\operatorname{ev}_{\iota_{h}}\left(b_{0}\right) \forall h \in I_{1}\right\}\right) / \operatorname{Aut}^{*}(\mathcal{T}),
\end{aligned}
$$

where the group $\operatorname{Aut}^{*}(\mathcal{T})$ is defined by

$$
\operatorname{Aut}^{*}(\mathcal{T})=\operatorname{Aut}(\mathcal{T}) /\left\{g \in \operatorname{Aut}(\mathcal{T}): g \cdot h=h \forall h \in I_{1}\right\}
$$

This decomposition is illustrated in Figure 5. In this figure, we represent an entire stratum of bubble maps by the domain of the stable maps in that stratum. The right-hand side of Figure 5 represents the subset of the cartesian product of the three spaces of bubble maps, corresponding to the three drawings, on which the appropriate evaluation maps agree pairwise, as indicated by the dotted lines and defined in (2-6).

Let $\mathcal{F} \mathcal{T} \longrightarrow \mathcal{U}_{\mathcal{T}}(X ; J)$ be the bundle of gluing parameters, or of smoothings at the nodes. This orbi-bundle has the form

$$
\mathcal{F} \mathcal{T}=\left(\bigoplus_{(h, i) \in \mathcal{N}} L_{h, 0} \otimes L_{i, 1} \oplus \bigoplus_{h \in \widehat{I}} L_{h, 0} \otimes L_{h, 1}\right) / \operatorname{Aut}(\mathcal{T}),
$$

for certain line orbi-bundles $L_{h, 0}$ and $L_{h, 1}$. Similarly to the genus-zero case,

$$
\mathcal{U}_{\mathcal{T}}(X ; J)=\mathcal{U}_{\mathcal{T}}^{(0)}(X ; J) / \operatorname{Aut}(\mathcal{T}) \propto\left(S^{1}\right)^{\hat{I}},
$$



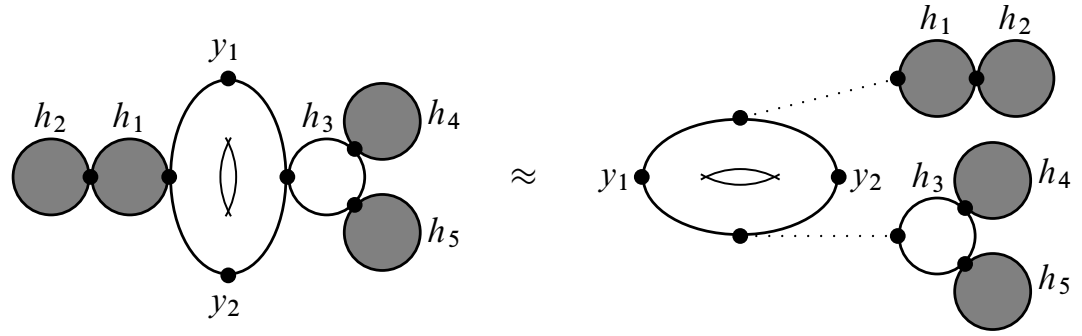

Figure 5: An example of the decomposition (2-6)

where

$$
\begin{aligned}
\mathcal{U}_{\mathcal{T}}^{(0)}(X ; J)=\left\{\left(b_{0},\left(b_{h}\right)_{h \in I_{1}}\right) \in \mathcal{U}_{\mathcal{T}_{0}}(X ; J) \times \prod_{h \in I_{1}} \mathcal{U}_{\mathcal{T}_{h}}^{(0)}(X ; J):\right. & \\
& \left.\quad \operatorname{ev}_{0}\left(b_{h}\right)=\operatorname{ev}_{\iota_{h}}\left(b_{0}\right) \forall h \in I_{1}\right\}
\end{aligned}
$$

and $\mathcal{U}_{\mathcal{T}_{h}}^{(0)}(X ; J)$ is the subspace of the moduli space of holomorphic maps from genus-zero curves as in Section 2.1. The line bundles $L_{h, 0}$ and $L_{h, 1}$ arise from the quotient (2-7). More precisely,

$\mathcal{F} \mathcal{T}=\widetilde{\mathcal{F}} \mathcal{T} / \operatorname{Aut}(\mathcal{T}) \propto\left(S^{1}\right)^{\hat{I}}, \quad$ where $\widetilde{\mathcal{F}} \mathcal{T}=\widetilde{\mathcal{F}}_{\boldsymbol{\aleph}} \mathcal{T} \oplus \widetilde{\mathcal{F}}_{0} \mathcal{T} \oplus \widetilde{\mathcal{F}}_{1} \mathcal{T} \longrightarrow \mathcal{U}_{\mathcal{T}}^{(0)}(X ; J)$,

$$
\widetilde{\mathcal{F}}_{0} \mathcal{T}=\bigoplus_{h \in I_{1}} \widetilde{\mathcal{F}}_{h} \mathcal{T}, \quad \widetilde{\mathcal{F}}_{1} \mathcal{T}=\mathcal{U}_{\mathcal{T}}^{(0)}(X ; J) \times \mathbb{C}^{\widehat{I}-I_{1}},
$$

and $\widetilde{\mathcal{F}}_{h} \mathcal{T}$ and $\widetilde{\mathcal{F}}_{\mathcal{N}} \mathcal{T}$ are the pullbacks by the projection map

$$
\pi_{P}: \mathcal{U}_{\mathcal{T}}^{(0)}(X ; J) \longrightarrow \mathcal{U}_{\mathcal{T}_{0}}(X ; J)
$$

of the universal tangent line $L_{h} \mathcal{T}_{0}$ at the $h$-th marked point and of the bundle $\mathcal{F} \mathcal{T}_{0}$ of gluing parameters. In other words, if $\mathfrak{U}_{\mathcal{T}_{0}} \longrightarrow \mathcal{U}_{\mathcal{T}_{0}}(X ; J)$ is the semiuniversal family, ie the fiber at $b_{0} \in \mathcal{U}_{\mathcal{T}_{0}}(X ; J)$ is the Riemann surface $\Sigma_{b_{0}}=\Sigma_{b_{0}, \boldsymbol{N}}, L_{h} \mathcal{T}_{0}$ is the vertical tangent space at the point $x_{h}\left(b_{0}\right)$ of $\Sigma_{b_{0}}$.

Remark 1 The above description is slightly inaccurate. In order to insure the existence of the space $\mathfrak{U}_{\mathcal{T}_{0}}$, with the fibers as described, we need to replace the space $\mathcal{U}_{\mathcal{T}_{0}}(X ; J)$ by a finite cover, analogous to the one used in [10]. However, correcting this inaccuracy would complicate the notation used even further, but would have no effect on the analysis, and thus we ignore it.

Remark 2 The rank of the bundle $\mathcal{F} \mathcal{T}_{0}$ is $|\boldsymbol{\aleph}|$, the number of nodes in the domain of every element of $\mathcal{U}_{\mathcal{T}_{0}}(X ; J)$. If $\boldsymbol{\aleph} \neq \varnothing, \mathcal{F} \mathcal{T}_{0}$ can be written as the quotient of 
the trivial bundle of rank $|\boldsymbol{\aleph}|$, over a space $\mathcal{U}_{\mathcal{T}_{0}}^{(0)}(X ; J)$, by an $\operatorname{Aut}\left(\mathcal{T}_{0}\right) \propto\left(S^{1}\right)^{\aleph}$-action in a manner similar to the previous subsection and to Section 2 of [16]. With the above identifications, the singular points of every rational component $\Sigma_{i}$ of $\Sigma_{\boldsymbol{N}}$ are the points 0 and $\infty$ in $S^{2}$. Thus, the equivalence class of the restriction of a stable map in $\mathcal{U}_{\mathcal{T}_{0}}(X ; J)$ to $\Sigma_{i}$, with its nodes, has a $\mathbb{C}^{*}$-family of representatives. This family is cut down to an $S^{1}$-family by restricting to the subset defined by the condition (B2) of Section 2.5 in [16]. This is the preimage of $1 / 2$ under the last, real-valued, component of the function $\Psi_{\mathcal{T}, i^{*}}$ defined in (2) of the proof of Proposition 3.3 in [16].

If $\mathcal{T}=(M, I, \mathfrak{\aleph} ; j, \underline{A})$ is a bubble type such that $A_{i}=0$ for all minimal elements $i$ of $I$, ie

$$
\mathcal{U}_{\mathcal{T}}(X ; J) \subset \overline{\mathfrak{M}}_{1, M}(X, A ; J)-\mathfrak{M}_{1, M}^{\{0\}}(X, A ; J), \quad \text { where } \quad A=\sum_{i \in I} A_{i},
$$

it is again useful to define a thickening of the set $\mathcal{U}_{\mathcal{T}}^{(0)}(X ; J)$. Thus, we put

$$
\begin{aligned}
\tilde{\mathcal{U}}_{\mathcal{T}}^{(0)}(X ; J)=\left\{\left(b_{0},\left(b_{h}\right)_{h \in I_{1}}\right) \in \mathcal{U}_{\mathcal{T}_{0}}(X ; J) \times\right. & \prod_{h \in I_{1}} \tilde{\mathcal{U}}_{\mathcal{T}_{h}}^{(0)}(X ; J): \\
& \left.\quad \operatorname{ev}_{0}\left(b_{h}\right)=\operatorname{ev}_{\iota_{h}}\left(b_{0}\right) \forall h \in I_{1}\right\},
\end{aligned}
$$

where the space $\tilde{\mathcal{U}}_{\mathcal{T}_{h}}^{(0)}(X ; J)$ is as in Section 2.1. Let

$$
\widetilde{\mathcal{F} \mathcal{T}}=\widetilde{\mathcal{F}_{\mathcal{N}} \mathcal{T}} \oplus \widetilde{\mathcal{F}_{0} \mathcal{T}} \oplus \widetilde{\mathcal{F}_{1} \mathcal{T}} \longrightarrow \widetilde{\mathcal{U}}_{\mathcal{T}}^{(0)}(X ; J),
$$

where

$$
\widetilde{\mathcal{F}_{\aleph} \mathcal{T}}=\tilde{\pi}_{P}^{*} \mathcal{F} \mathcal{T}_{0}, \widetilde{\mathcal{F}_{0} \mathcal{T}}=\bigoplus_{h \in I_{1}} \widetilde{\mathcal{F}_{h} \mathcal{T}}, \widetilde{\mathcal{F}_{h} \mathcal{T}}=\tilde{\pi}_{P}^{*} L_{h} \mathcal{T}_{0}, \widetilde{\mathcal{F}_{1} \mathcal{T}}=\tilde{\mathcal{U}}_{\mathcal{T}}^{(0)}(X ; J) \times \mathbb{C}^{\widehat{I}-I_{1}},
$$

and $\tilde{\pi}_{P}: \tilde{\mathcal{U}}_{\mathcal{T}}^{(0)}(X ; J) \longrightarrow \mathcal{U}_{\mathcal{T}_{0}}(X ; J)$ is the projection map. As before, we denote by $\widetilde{\mathcal{F} \mathcal{T}} \varnothing$ the subset of $\widetilde{\mathcal{F} \mathcal{T}}$ consisting of the elements with all components nonzero.

Suppose $\mathcal{T}=(M, I, \aleph ; j, \underline{A})$ is a bubble type as in the previous paragraph. Since every holomorphic map in the zero homology class is constant, the decomposition (2-6) is equivalent to

$$
\begin{aligned}
\mathcal{U}_{\mathcal{T}}(X ; J) & \approx\left(\mathcal{U}_{\mathcal{T}_{0}}(\mathrm{pt}) \times \mathcal{U}_{\overline{\mathcal{T}}}(X ; J)\right) / \operatorname{Aut}^{*}(\mathcal{T}) \\
& \subset\left(\overline{\mathcal{M}}_{1, k_{0}} \times \mathcal{U}_{\overline{\mathcal{T}}}(X ; J)\right) / \operatorname{Aut}^{*}(\mathcal{T}),
\end{aligned}
$$


where $k_{0}=\left|M_{0}\right|+\left|I_{1}\right|, \overline{\mathcal{M}}_{1, k_{0}}$ is the moduli space of genus-one curves with $k_{0}$ marked points, and

$$
\mathcal{U}_{\overline{\mathcal{T}}}(X ; J)=\left\{\left(b_{h}\right)_{h \in I_{1}} \in \prod_{h \in I_{1}} \mathcal{U}_{\mathcal{T}_{h}}(X ; J): \operatorname{ev}_{0}\left(b_{h_{1}}\right)=\operatorname{ev}_{0}\left(b_{h_{2}}\right) \forall h_{1}, h_{2} \in I_{1}\right\} .
$$

Similarly, (2-9) is equivalent to

$$
\tilde{\mathcal{U}}_{\mathcal{T}}^{(0)}(X ; J) \approx \mathcal{U}_{\mathcal{T}_{0}}(\mathrm{pt}) \times \tilde{\mathcal{U}}_{\overline{\mathcal{T}}}^{(0)}(X ; J) \subset \overline{\mathcal{M}}_{1, k_{0}} \times \tilde{\mathcal{U}}_{\overline{\mathcal{T}}}^{(0)}(X ; J),
$$

where

$$
\begin{aligned}
& \tilde{\mathcal{U}}_{\overline{\mathcal{T}}}^{(0)}(X ; J) \\
& \quad=\left\{\left(b_{h}\right)_{h \in I_{1}} \in \prod_{h \in I_{1}} \tilde{\mathcal{U}}_{\mathcal{T}_{h}}^{(0)}(X ; J): \operatorname{ev}_{0}\left(b_{h_{1}}\right)=\operatorname{ev}_{0}\left(b_{h_{2}}\right) \forall h_{1}, h_{2} \in I_{1}\right\} .
\end{aligned}
$$

We denote by

$$
\begin{aligned}
\pi_{P}: \mathcal{U}_{\mathcal{T}}(X ; J) \longrightarrow & \overline{\mathcal{M}}_{1, k_{0}} / \operatorname{Aut}^{*}(\mathcal{T}), \quad \tilde{\pi}_{P}: \tilde{\mathcal{U}}_{\mathcal{T}}^{(0)}(X ; J) \longrightarrow \overline{\mathcal{M}}_{1, k_{0}}, \\
& \operatorname{ev}_{P}: \mathcal{U}_{\mathcal{T}}(X ; J), \tilde{\mathcal{U}}_{\mathcal{T}}^{(0)}(X ; J) \longrightarrow X
\end{aligned}
$$

the projections onto the first component in the decompositions (2-10) and (2-11) and the map sending each element $[\mathcal{C}, u]$ of $\mathcal{U}_{\mathcal{T}}(X ; J)$, or $(\mathcal{C}, u)$ of $\tilde{\mathcal{U}}_{\mathcal{T}}^{(0)}(X ; J)$, to the image of the principal component $\mathcal{C}_{P}$ of $\mathcal{C}$, ie the point $u\left(\mathcal{C}_{P}\right)$ in $X$.

Let $\mathbb{E} \longrightarrow \overline{\mathcal{M}}_{1, k_{0}}$ denote the Hodge line bundle, ie the line bundle of holomorphic differentials. For each $i \in \chi(\mathcal{T})$, we define the bundle map

$\mathcal{D}_{J, i}: \overline{\mathcal{F}_{h(i)} \mathcal{T}} \longrightarrow \tilde{\pi}_{P}^{*} \mathbb{E}^{*} \otimes \operatorname{ev}_{P}^{*} T X, \quad$ where $\quad h(i)=\min \{h \in \hat{I}: h \leq i\} \in I_{1}$, over $\tilde{\mathcal{U}}_{\mathcal{T}}^{(0)}(X ; J)$ by

$$
\left\{\mathcal{D}_{J, i}(\widetilde{v})\right\}(\psi)=\psi_{x_{h(i)}(b)}(\widetilde{v}) \cdot{ }_{J} \mathcal{D}_{i} b \in T_{\operatorname{ev}_{P}(b)} X
$$

if

$$
\psi \in \tilde{\pi}_{P}^{*} \mathbb{E}, \quad \tilde{v}=(b, \widetilde{v}) \in \widetilde{\mathcal{F}_{h(i)} \mathcal{T}}, b \in \tilde{\mathcal{U}}_{\mathcal{T}}^{(0)}(X ; J),
$$

and $x_{h(i)}(b) \in \Sigma_{b, \aleph}$ is the node joining the bubble $\Sigma_{b, h(i)}$ of $b$ to the principal component $\Sigma_{b, \aleph}$ of $\Sigma_{b}$. For each $v \in \widetilde{\mathcal{F} \mathcal{T}}$, we put

$$
\rho(v)=\left(b,\left(\rho_{i}(v)\right)_{i \in \chi(\mathcal{T})}\right) \in \bigoplus_{i \in \chi(\mathcal{T})} \widetilde{\mathcal{F}_{h(i)} \mathcal{T}}, \quad \text { where } \quad \rho_{i}(v)=\prod_{h \in \widehat{I}, h \leq i} v_{h} \in \widetilde{\mathcal{F}_{h(i)} \mathcal{T}},
$$

if $v=\left(b ; v_{\boldsymbol{\aleph}},\left(v_{i}\right)_{i \in \hat{I}}\right), b \in \tilde{\mathcal{U}}_{\mathcal{T}}^{(0)}(X ; J),\left(b, v_{\boldsymbol{\aleph}}\right) \in \widetilde{\mathcal{F}_{\boldsymbol{\aleph}} \mathcal{T}},\left(b, v_{h}\right) \in \widetilde{\mathcal{F}_{h} \mathcal{T}}$ if $h \in \widehat{I}_{1}$. 
These definitions are illustrated in Figure 6. While the restrictions of these bundle maps to $\mathcal{U}_{\mathcal{T}}^{(0)}(X ; J) \subset \tilde{\mathcal{U}}_{\mathcal{T}}^{(0)}(X ; J)$ do not necessarily descend to the vector bundle $\mathcal{F} \mathcal{T}$ over $\mathcal{U}_{\mathcal{T}}(X ; J)$, the map

$$
\mathcal{D}_{\mathcal{T}}: \mathcal{F} \mathcal{T} \longrightarrow \pi_{P}^{*} \mathbb{E}^{*} \otimes \operatorname{ev}_{P}^{*} T X / \operatorname{Aut}^{*}(\mathcal{T}), \quad \mathcal{D}_{\mathcal{T}}(v)=\sum_{i \in \chi(\mathcal{T})} \mathcal{D}_{J, i} \rho_{i}(v),
$$

is well-defined.

Finally, if $\mathcal{T}$ is any bubble type, for genus-zero or genus-one maps, and $K$ is a subset of $\mathcal{U}_{\mathcal{T}}(X ; J)$, we denote by $K^{(0)}$ and $\widetilde{K}^{(0)}$ the preimages of $K$ under the quotient projection maps

$$
\mathcal{U}_{\mathcal{T}}^{(0)}(X ; J) \longrightarrow \mathcal{U}_{\mathcal{T}}(X ; J) \quad \text { and } \quad \tilde{\mathcal{U}}_{\mathcal{T}}^{(0)}(X ; J) \longrightarrow \mathcal{U}_{\mathcal{T}}(X ; J),
$$

respectively. All vector orbi-bundles we encounter will be assumed to be normed. Some will come with natural norms; for others, we choose a norm, sometimes implicitly, once and for all. If $\pi_{\mathfrak{F}}: \mathfrak{F} \longrightarrow \mathfrak{X}$ is a normed vector bundle and $\delta: \mathfrak{X} \longrightarrow \mathbb{R}$ is any function, possibly constant, let

$$
\mathfrak{F}_{\delta}=\left\{v \in \mathfrak{F}:|v|<\delta\left(\pi_{\mathfrak{F}}(v)\right)\right\} .
$$

If $\Omega$ is any subset of $\mathfrak{F}$, we take $\Omega_{\delta}=\Omega \cap \mathfrak{F}_{\delta}$.

\subsection{Boundary structure theorem}

In this subsection, we formulate Theorem 2.3, which states that an element

$$
b \in \overline{\mathfrak{M}}_{1, k}(X, A ; J)-\mathfrak{M}_{1, k}^{\{0\}}(X, A ; J)
$$

lies in the stable-map closure of the space $\mathfrak{M}_{1, k}^{0}(X, A ; J)$ of genus-one $J$-holomorphic maps from smooth domains if and only if $b$ lies in $\overline{\mathfrak{M}}_{1, k}^{0}(X, A ; J)$, provided the almost complex structure $J$ is sufficiently regular. In addition, Theorem 2.3 describes a neighborhood of every stratum of

$$
\overline{\mathfrak{M}}_{1, k}^{0}(X, A ; J)-\mathfrak{M}_{1, k}^{\{0\}}(X, A ; J)
$$

in $\overline{\mathfrak{M}}_{1, k}^{0}(X, A ; J)$. If $k \in \overline{\mathbb{Z}}^{+}$, we denote by $[k]$ the set $\{1, \ldots, k\}$.

Theorem 2.3 Suppose $(X, \omega, J)$ is a compact almost Kahler manifold and $A \in$ $H_{2}(X ; \mathbb{Z})^{*}$. If the regularity conditions (a) and (b-i) of Definition 1.4 are satisfied and $\mathcal{T}=([k], I, \aleph ; j, \underline{A})$ is a bubble type such that $\sum_{i \in I} A_{i}=A$ and $A_{i}=0$ for all 
minimal elements $i$ of $I$, then the intersection of the closure of $\mathfrak{M}_{1, k}^{0}(X, A ; J)$ in $\overline{\mathfrak{M}}_{1, k}(X, A ; J)$ with $\mathcal{U}_{\mathcal{T}}(X ; J)$ is the set

$$
\mathcal{U}_{\mathcal{T} ; 1}(X ; J) \equiv\left\{[b] \in \mathcal{U}_{\mathcal{T}}(X ; J): \operatorname{dim}_{\mathbb{C}} \operatorname{Span}_{(\mathbb{C}, J)}\left\{\mathcal{D}_{i} b: i \in \chi(\mathcal{T})\right\}<|\chi(\mathcal{T})|\right\} .
$$

Furthermore, the space

$$
\mathcal{F}^{1} \mathcal{T}^{\varnothing} \equiv\left\{[v]=[b, v] \in \mathcal{F} \mathcal{T}^{\varnothing}: \mathcal{D}_{\mathcal{T}}(v)=0\right\}
$$

is a smooth oriented suborbifold of $\mathcal{F} \mathcal{T}$. Finally, there exist $\delta \in C\left(\mathcal{U}_{\mathcal{T}}(X ; J) ; \mathbb{R}^{+}\right)$, an open neighborhood $U_{\mathcal{T}}$ of $\mathcal{U}_{\mathcal{T}}(X ; J)$ in $\mathfrak{X}_{1, k}(X, A)$, and an orientation-preserving diffeomorphism

$$
\phi: \mathcal{F}^{1} \mathcal{T}_{\delta}^{\varnothing} \longrightarrow \mathfrak{M}_{1, k}^{0}(X, A ; J) \cap U_{\mathcal{T}},
$$

which extends to a homeomorphism

$$
\phi: \mathcal{F}^{1} \mathcal{T}_{\delta} \longrightarrow \overline{\mathfrak{M}}_{1, k}^{0}(X, A ; J) \cap U_{\mathcal{T}},
$$

where $\mathcal{F}^{1} \mathcal{T}$ is the closure of $\mathcal{F}^{1} \mathcal{T}^{\varnothing}$ in $\mathcal{F} \mathcal{T}$.

We now clarify the statement of Theorem 2.3 and illustrate it using Figure 6. As before, the shaded discs represent the components of the domain on which every stable map $[b]$ in $\mathcal{U}_{\mathcal{T}}(X ; J)$ is nonconstant. A stable map

$$
[\mathcal{C}, u] \in \mathcal{U}_{\mathcal{T}}(X ; J) \subset \overline{\mathfrak{M}}_{1, k}(X, A ; J)-\mathfrak{M}_{1, k}^{\{0\}}(X, A ; J)
$$

is in the stable-map closure of $\mathfrak{M}_{1, k}^{0}(X, A ; J)$ if and only if $[\mathcal{C}, u]$ satisfies condition (b) of Definition 1.1.

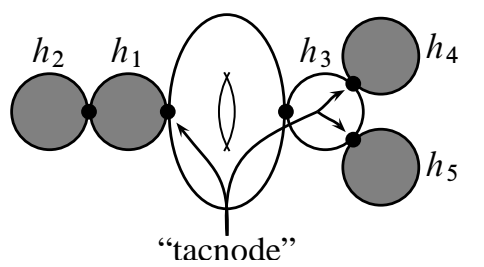

$$
\begin{aligned}
& \chi(\mathcal{T})=\{\left.h_{1}, h_{4}, h_{5}\right\}, \rho(v)=\left(v_{h_{1}}, v_{h_{3}} v_{h_{4}}, v_{h_{3}} v_{h_{5}}\right) \\
& \mathcal{F}^{1} \mathcal{T}^{\emptyset}=\left\{\left[b ; v_{h_{1}}, v_{h_{2}}, v_{h_{3}}, v_{h_{4}}, v_{h_{5}}\right]: v_{h_{2}}, v_{h_{4}}, v_{h_{5}} \in \mathbb{C}^{*} ;\right. \\
& v_{h_{1}} \in T_{x_{h_{1}}} \Sigma_{P}-\{0\}, v_{h_{3}} \in T_{x_{h_{3}}} \Sigma_{P}-\{0\} ; \\
&\left.v_{h_{1}} \mathcal{D}_{J, h_{1}} b+v_{h_{3}} v_{h_{4}} \mathcal{D}_{J, h_{4}} b+v_{h_{3}} v_{h_{5}} \mathcal{D}_{J, h_{5}} b=0\right\}
\end{aligned}
$$

Figure 6: An illustration of Theorem 2.3

Standard arguments show that the regularity condition (a) of Definition 1.4 implies that the space $\mathcal{U}_{\mathcal{T}}^{(0)}(X ; J)$ is a smooth manifold, while $\mathcal{U}_{\mathcal{T}}(X ; J)$ is a smooth orbifold; see Chapter 3 in [8], for example. Thus, the total space of the bundle $\mathcal{F} \mathcal{T}^{\varnothing}$ is also a 
smooth orbifold. The second claim of Theorem 2.3 is immediate from the transversality of the bundle map

$$
\mathcal{D}_{\mathcal{T}}: \mathcal{F} \mathcal{T}^{\varnothing} \longrightarrow \pi_{P}^{*} \mathbb{E}^{*} \otimes \operatorname{ev}_{P}^{*} T X / \operatorname{Aut}^{*}(\mathcal{T}), \quad \mathcal{D}_{\mathcal{T}}(v)=\sum_{i \in \chi(\mathcal{T})} \mathcal{D}_{J, i} \rho_{i}(v),
$$

to the zero set. In turn, this transversality property is an immediate consequence of the regularity conditions (a) and (b-i) of Definition 1.4.

The middle claim of Theorem 2.3 is needed to make sense of the remaining statement. This final claim, proved in Section 6, describes a normal neighborhood of $\mathcal{U}_{\mathcal{T} ; 1}(X ; J)$ in $\overline{\mathfrak{M}}_{1, k}^{0}(X, A ; J)$ and implies the first statement of Theorem 2.3.

Remark The regularity assumptions on $J$ used in Theorem 2.3 do not guarantee that the entire space $\mathfrak{M}_{1, k}^{0}(X, A ; J)$ is smooth. However, the proof of Theorem 1.6 implies that $\mathfrak{M}_{1, k}^{0}(X, A ; J)$ is smooth near each stratum $\mathcal{U}_{\mathcal{T} ; 1}(X ; J)$ of $\overline{\mathfrak{M}}_{1, k}^{0}(X, A ; J)$. This can be seen from the $\widetilde{J}=J$ case of Corollary 4.5 and standard Implicit Function Theorem arguments such as those in Chapter 3 of [8].

Proof of Corollary 1.5 It remains to construct a fundamental class for $\overline{\mathfrak{M}}_{1, k}^{0}(X, A ; J)$. Theorem 2.3 gives a description of a neighborhood in $\overline{\mathfrak{M}}_{1, k}^{0}(X, A ; J)$ of every stratum $\overline{\mathfrak{M}}_{1, k}^{0}(X, A ; J) \cap \mathcal{U}_{\mathcal{T}}(X ; J)$ for a bubble type $\mathcal{T}=(M, I, \boldsymbol{\aleph} ; j, \underline{A})$ such that $A_{i}=0$ for all minimal elements $i \in I$. If $\mathcal{T}$ is a bubble type such that $A_{i} \neq 0$ for some minimal element $i \in I$, a neighborhood of

$$
\overline{\mathfrak{M}}_{1, k}^{0}(X, A ; J) \cap \mathcal{U}_{\mathcal{T}}(X ; J)=\mathcal{U}_{\mathcal{T}}(X ; J)
$$

in $\overline{\mathfrak{M}}_{1, k}^{0}(X, A ; J)$ is homeomorphic to a neighborhood of $\mathcal{U}_{\mathcal{T}}(X ; J)$ in the corresponding bundle of gluing parameters $\mathcal{F} \mathcal{T}$, as can be seen from Section 4.1 and the continuity arguments of [16, Sections 3.9]. Since

$$
\partial \overline{\mathfrak{M}}_{1, k}^{0}(X, A ; J) \equiv \overline{\mathfrak{M}}_{1, k}^{0}(X, A ; J)-\mathfrak{M}_{1, k}^{0}(X, A ; J)
$$

is a union of smooth orbifolds of (real) dimension at most $2\left(\left\langle c_{1}(T X), A\right\rangle+k\right)-2$, it follows that there exist arbitrary small neighborhoods $U$ of $\partial \overline{\mathfrak{M}}_{1, k}^{0}(X, A ; J)$ such that

$$
H_{l}(U ; \mathbb{Q})=\{0\} \quad \forall l \geq 2\left(\left\langle c_{1}(T X), A\right\rangle+k\right)-1 ;
$$

see [20, Section 2.2]. Since the moduli space $\mathfrak{M}_{1, k}^{0}(X, A ; J)$ is a smooth oriented orbifold,

$$
\operatorname{dim}_{\mathbb{R}} \mathfrak{M}_{1, k}^{0}(X, A ; J)=2\left(\left\langle c_{1}(T X), A\right\rangle+k\right),
$$


and the complement of $U$ in $\overline{\mathfrak{M}}_{1, k}^{0}(X, A ; J)$ is compact, $\mathfrak{M}_{1, k}^{0}(X, A ; J)$ determines a class

$$
\begin{aligned}
& {\left[\overline{\mathfrak{M}}_{1, k}^{0}(X, A ; J)\right] \in H_{2\left(\left\langle c_{1}(T X), A\right\rangle+k\right)}\left(\overline{\mathfrak{M}}_{1, k}^{0}(X, A ; J), U ; \mathbb{Q}\right)} \\
& \quad \approx H_{2\left(\left\langle c_{1}(T X), A\right\rangle+k\right)}\left(\overline{\mathfrak{M}}_{1, k}^{0}(X, A ; J) ; \mathbb{Q}\right),
\end{aligned}
$$

as claimed. The isomorphism between the two homology groups is induced by inclusion.

\section{A genus-zero gluing procedure}

\subsection{The genus-zero regularity properties}

In this subsection, we prove the $g=0$ case of the first claim of Theorem 1.6. It follows from Corollary 3.2 and the compactness of the moduli space $\overline{\mathfrak{M}}_{0,1}(X, A ; J)$. Corollary 3.2 is obtained by a rather straightforward argument via the analytic part of [6]. Throughout this subsection, we assume that $J$ is a genus-zero $A$-regular almost complex structure on $X$.

In order to prove Theorem 1.6, we need to describe smooth maps $u$ : $\mathbb{P}^{1} \longrightarrow X$, with one or two marked points, that lie close to each stratum $\mathcal{U}_{\mathcal{T}}(X ; J)$ of $\overline{\mathfrak{M}}_{0,1}(X, A ; J)$ and of

$$
\overline{\mathfrak{M}}_{0,2}(X, A ; J) \approx \overline{\mathfrak{M}}_{0,\{0\} \sqcup\{1\}}(X, A ; J) .
$$

We denote by $\mathfrak{X}_{0, M}(X, A)$ the space of equivalence classes of all smooth maps into $X$ from genus-zero Riemann surfaces with marked points indexed by the set $\{0\} \sqcup M$ in the homology class $A$ and by $\mathfrak{X}_{0, M}^{0}(X, A)$ the subset of $\mathfrak{X}_{0, M}(X, A)$ consisting of the maps with smooth domains, ie $\mathbb{P}^{1}$ in this case.

Let $\mathcal{T}=(M, I ; j, \underline{A})$ be a bubble type such that $\sum_{i \in I} A_{i}=A$, ie $\mathcal{U}_{\mathcal{T}}(X ; J)$ is a stratum of the moduli space $\overline{\mathfrak{M}}_{0,\{0\} \sqcup M}(X, A ; J)$. We will proceed as in Sections 3.3 and 3.6 of [16]. Sections 2.1 and 2.3 in [13] describe a special case of the same construction in circumstances very similar to the present situation.

For each sufficiently small element $v=(b, v)$ of $\widetilde{\mathcal{F} \mathcal{T}} \varnothing$, where $b=\left(\Sigma_{b}, u_{b}\right)$ is an element of $\tilde{\mathcal{U}}_{\mathcal{T}}^{(0)}(X ; J)$, let

$$
q_{v}: \Sigma_{v} \longrightarrow \Sigma_{b}
$$

be the basic gluing map constructed in Section 2.2 of [16]. In this case, $\Sigma_{v}$ is the projective line $\mathbb{P}^{1}$ with $|M|+1$ marked points. Let

$$
b(v)=\left(\Sigma_{v}, u_{v}\right), \quad \text { where } \quad u_{v}=u_{b} \circ q_{v},
$$


be the approximately holomorphic map corresponding to $v$. The primary marked point $y_{0}(v)$ of $\Sigma_{v}$ is the point $\infty$ of $\Sigma_{v} \approx S^{2}$.

Let $\nabla^{J}$ be the $J$-linear connection induced by the Levi-Civita connection of the metric $g_{X}$. Since the linearization $D_{J, b}$ of the $\bar{\partial}_{J}$-operator at $b$ is surjective by Definition 1.3, if $v \in \widetilde{\mathcal{F} \mathcal{T}} \varnothing$ is sufficiently small, the linearization

$$
D_{J, v}: \Gamma(v) \equiv L_{1}^{p}\left(\Sigma_{v} ; u_{v}^{*} T X\right) \longrightarrow \Gamma^{0,1}(v ; J) \equiv L^{p}\left(\Sigma_{v} ; \Lambda_{J, j}^{0,1} T^{*} \Sigma_{v} \otimes u_{v}^{*} T X\right)
$$

of the $\bar{\partial}_{J}$-operator at $b(v)$, defined via the connection $\nabla^{J}$, is also surjective. In particular, we can obtain a decomposition

$$
\Gamma(v)=\Gamma_{-}(v) \oplus \Gamma_{+}(v)
$$

such that the linear operator $D_{J, v}: \Gamma_{+}(v) \longrightarrow \Gamma^{0,1}(v ; J)$ is an isomorphism, while

$$
\Gamma_{-}(v)=\left\{\xi \circ q_{v}: \xi \in \Gamma_{-}(b) \equiv \operatorname{ker} D_{J, b}\right\} .
$$

For the purposes of this subsection, the space $\Gamma_{+}(v)$ can be taken to be the $L^{2}-$ orthogonal complement of $\Gamma_{-}(v)$, but for use in later subsections it is more convenient to take

$$
\begin{aligned}
\Gamma_{+}(v)=\{\zeta \in \Gamma(v) & : \zeta(\widehat{0}, \infty)=0 \\
& \left.\langle\zeta, \xi\rangle\rangle_{v, 2}=0 \forall \xi \in \Gamma_{-}(v) \text { such that } \xi(\widehat{0}, \infty)=0\right\}
\end{aligned}
$$

where $(\hat{0}, \infty)$ is the primary marked point, ie the south pole of the sphere $\Sigma_{v} \approx S^{2}$. This choice of $\Gamma_{+}(v)$ is permissible by Definition 1.3. The $L^{2}$-inner product on $\Gamma(v)$ used in (3-2) is defined via the metric $g_{X}$ on $X$ and the metric $g_{v}$ on $\Sigma_{v}$ induced by the pregluing construction. The Banach spaces $\Gamma(v)$ and $\Gamma^{0,1}(v ; J)$ carry the norms $\|\cdot\|_{v, p, 1}$ and $\|\cdot\|_{v, p}$, respectively, which are also defined by the pregluing construction. Throughout this paper, $p$ denotes a real number greater than two. The norms $\|\cdot\|_{v, p, 1}$ and $\|\cdot\|_{v, p}$ are equivalent to the ones used in [6]. In particular, the norms of $D_{J, v}$ and of the inverse of its restriction to $\Gamma_{+}(v)$ have fiberwise uniform upper bounds, ie dependent only on $[b] \in \mathcal{U}_{\mathcal{T}}(X ; J)$, and not on $v \in \mathbb{C}^{* \widehat{I}}$. We denote by

$$
\pi_{v ;-}: \Gamma(v) \longrightarrow \Gamma_{-}(v) \quad \text { and } \quad \pi_{v ;+}: \Gamma(v) \longrightarrow \Gamma_{+}(v)
$$

the projection maps corresponding to the decomposition (3-1). The relevant facts concerning the objects described in this paragraph are summarized in Lemma 3.1:

Lemma 3.1 Let $(X, \omega, J)$ be a compact almost Kahler manifold and $A \in H_{2}(X ; \mathbb{Z})$. If $J$ is a genus-zero $A$-regular almost complex structure and $\mathcal{T}=(M, I ; j, \underline{A})$ is a bubble type such that $A=\sum_{i \in I} A_{i}$, there exist $\delta, C \in C\left(\mathcal{U}_{\mathcal{T}}(X ; J) ; \mathbb{R}^{+}\right)$and an open neighborhood $U_{\mathcal{T}}$ of $\mathcal{U}_{\mathcal{T}}(X ; J)$ in $\mathfrak{X}_{0, M}(X, A)$ with the following properties: 
(1) For all $v=(b, v) \in \widetilde{\mathcal{F}}_{\delta}^{\varnothing}$,

$$
\begin{aligned}
\left\|\pi_{v ;-} \xi\right\|_{v, p, 1} & \leq C(b)\|\xi\|_{v, p, 1} & & \forall \xi \in \Gamma(v), \\
\left\|D_{J, v} \xi\right\|_{v, p} & \leq C(b)|v|^{1 / p}\|\xi\|_{v, p, 1} & & \forall \xi \in \Gamma_{-}(v), \\
C(b)^{-1}\|\xi\|_{v, p, 1} & \leq\left\|D_{J, v} \xi\right\|_{v, p} \leq C(b)\|\xi\|_{v, p, 1} & & \forall \xi \in \Gamma_{+}(v) ;
\end{aligned}
$$

(2) For every $[\tilde{b}] \in \mathfrak{X}_{0, M}^{0}(X, A) \cap U_{\mathcal{T}}$, there exist $v \in \widetilde{\mathcal{F}} \mathcal{T}_{\delta}^{\varnothing}$ and $\zeta \in \Gamma_{+}(v)$ such that $\|\zeta\|_{v, p, 1}<\delta(b)$ and $\left[\exp _{b(v)} \zeta\right]=[\tilde{b}]$.

The first two bounds in (1) follow immediately from the definition of the spaces $\Gamma_{-}(v)$. The third estimate can be deduced from the facts that

$$
\begin{gathered}
\|\xi\|_{v, p, 1} \leq C(b)\left(\left\|D_{J, v} \xi\right\|_{v, p}+\|\xi\|_{v, p}\right),\|\xi\|_{C^{0}} \leq C(b)\|\xi\|_{v, p, 1} \forall \xi \in \Gamma(v) \\
\lim _{v \longrightarrow b} \Gamma_{-}(v)=\Gamma_{-}(b) \quad \text { if } \quad b=\left(\Sigma_{b}, u_{b}\right) \in \tilde{\mathcal{U}}_{\mathcal{T}}^{(0)}(X ; J)
\end{gathered}
$$

see Section 3.5 in [16]. In (2) of Lemma 3.1, $\exp _{b(v)} \zeta$ denotes the stable map that has the same domain and marked points as the map $b(v)$, but the map into $X$ is $\exp _{u_{v}} \zeta$, where exp is the exponential map of the connection $\nabla^{J}$. The final claim of Lemma 3.1 also follows from the above properties of $\Gamma_{-}(v)$, along with the uniformly smooth dependence of the spaces $\Gamma_{-}(v)$ on $v$; see Section 4 of [16]. In fact, for each $[\tilde{b}]$ in $U_{\mathcal{T}} \cap \mathfrak{X}_{0, M}^{0}(X, A)$, the corresponding pair $(v, \zeta)$ is unique, up to the action of the $\operatorname{group} \operatorname{Aut}(\mathcal{T}) \propto\left(S^{1}\right)^{I}$.

Corollary 3.2 If $(X, \omega, J), A$, and $\mathcal{T}$ are as in Lemma 3.1 and $M=\varnothing$, for every precompact open subset $K$ of $\mathcal{U}_{\mathcal{T}}(X ; J)$, there exist $\delta_{K}, C_{K} \in \mathbb{R}^{+}$and an open neighborhood $U_{K} \subset U_{\mathcal{T}}$ of $K$ in $\mathfrak{X}_{0, \varnothing}(X, A)$ with the following properties:

(1) Requirements (1) and (2) of Lemma 3.1 are satisfied.

(2) If $\tilde{J}$ is an almost complex structure on $X$ such that $\|\tilde{J}-J\|_{C^{1}}<\delta_{K}$ and $[\tilde{b}] \in U_{K} \cap \mathfrak{X}_{0,1}^{0}(X, A)$, there exists a smooth map $\tilde{u}: \mathbb{P}^{1} \longrightarrow X$ such that $[\tilde{b}]=\left[\mathbb{P}^{1}, \tilde{u}\right]$ and, for a choice of linearization of $\bar{\partial}_{\widetilde{J}}$ at $\tilde{u}$, the operators $D_{\widetilde{J}, \tilde{u}}$ and $\mathfrak{D}_{\widetilde{J}, \widetilde{u}}^{\infty}$ are surjective.

Remark If the map $\tilde{u}$ is $\widetilde{J}$-holomorphic, ie $\bar{\partial} \widetilde{J}$ vanishes at $\tilde{u}$, there is only one linearization of $\bar{\partial} \tilde{J}$ at $\tilde{u}$, though there are different ways of writing it explicitly. In the proof of this corollary, whether or not $\tilde{u}$ is a $\widetilde{J}$-holomorphic map, $D_{\widetilde{J}, \widetilde{u}}$ denotes the linearization of $\bar{\partial} \tilde{J}$ at $\tilde{u}$ with respect to the connection $\nabla^{\widetilde{J}}$; see [8, Chapter 3]. 
Proof (1) By (2) of Lemma 3.1, it is sufficient to check the surjectivity claims for every smooth map $\tilde{u}=\exp _{u_{v}} \zeta$, where $v=\left.(b, v) \in \tilde{\mathcal{F}} \mathcal{T}_{\delta_{K}}^{\varnothing}\right|_{K^{(0)}}$ and $\|\zeta\|_{v, p, 1}<\delta_{K}$. If

$$
\xi \in \Gamma(\widetilde{u}) \equiv L_{1}^{p}\left(\Sigma_{v} ; \tilde{u}^{*} T X\right)
$$

we define $\tilde{\xi} \in \Gamma(v)$ by

$$
\tilde{\xi}(z)=\Pi_{\zeta(z)}^{-1} \xi(z) \quad \forall z \in \mathbb{P}^{1},
$$

where $\Pi_{\zeta(z)}$ is the parallel transport along the geodesic $t \longrightarrow \exp _{u_{v}(z)} t \zeta(z)$ with respect to $\nabla^{J}$. By (3-3),

$$
\begin{aligned}
&\left\|D_{J, v} \tilde{\xi}\right\|_{v, p} \leq\left\|D_{\widetilde{J}, \tilde{u}} \xi\right\|_{v, p}+C_{K}\left(\|\widetilde{J}-J\|_{C^{1}}+\|\zeta\|_{v, p, 1}\right)\|\xi\|_{v, p, 1} \\
&=\left\|D_{\widetilde{J}, \tilde{u}} \xi\right\|_{v, p}+C_{K}\left(\|\widetilde{J}-J\|_{C^{1}}+\|\zeta\|_{v, p, 1}\right)\|\tilde{\xi}\|_{v, p, 1} \\
& \forall \xi \in \Gamma(\widetilde{u}) .
\end{aligned}
$$

Thus, by (1) of Lemma 3.1,

$$
\begin{aligned}
\left\|\pi_{v ;+} \tilde{\xi}\right\|_{v, p, 1} & \leq C_{K}^{\prime}\left(\|\tilde{J}-J\|_{C^{1}}+\|\zeta\|_{v, p, 1}\right)\|\tilde{\xi}\|_{v, p, 1} \quad \forall \xi \in \operatorname{ker} D_{\widetilde{J}, \tilde{u}}, \\
\Longrightarrow \quad\|\tilde{\xi}\|_{v, p, 1} & \leq C_{K}\left\|\pi_{v ;-} \tilde{\xi}\right\|_{v, p, 1}
\end{aligned}
$$

if $\delta_{K}$ is sufficiently small. By (3-5) and (a) of Definition 1.3,

$$
\operatorname{dim} \operatorname{ker} D_{\widetilde{J}, \tilde{u}} \leq \operatorname{dim} \Gamma_{-}(v)=\operatorname{ind} D_{J, b}=\operatorname{ind} D_{\widetilde{J}, \tilde{u}}
$$

In particular, the operator $D_{\widetilde{J}, \widetilde{u}}$ is surjective.

(2) The surjectivity of the map $\mathfrak{D}_{\widetilde{J}, \widetilde{u}}^{\infty}$ is proved similarly. Let

$$
\begin{aligned}
& \tilde{\pi}_{v ;-}: \Gamma_{-}(v) \longrightarrow \tilde{\Gamma}_{-}(v) \equiv\left\{\xi \in \Gamma_{-}(v): \xi(\infty)=0\right\} \approx \operatorname{ker} \mathfrak{D}_{J, u_{b}}^{\infty}, \\
& \tilde{\pi}_{v ;+}: \Gamma_{-}(v) \longrightarrow \tilde{\Gamma}_{+}(v) \equiv\left\{\xi \in \Gamma_{-}(v):\left\langle\left\langle\xi, \xi^{\prime}\right\rangle\right\rangle_{v, 2}=0 \forall \xi^{\prime} \in \tilde{\Gamma}_{-}(v)\right\}
\end{aligned}
$$

be the $L^{2}$-orthogonal projections onto $\widetilde{\Gamma}_{-}(v)$ and its orthogonal complement in $\Gamma_{-}(v)$. Then,

$$
\|\xi\|_{v, p, 1} \leq C_{K}|\xi(\infty)| \quad \forall \xi \in \widetilde{\Gamma}_{+}(v)
$$

since the analogous bound holds for the map

$$
\mathfrak{D}_{J, b}^{\infty}: \operatorname{ker} D_{J, u} \longrightarrow T_{u(\infty)} X,
$$


by Definition 1.3. Combining (3-3)-(3-6), we obtain

$$
\begin{aligned}
\left\|\tilde{\pi}_{v ;+} \pi_{v ;-} \tilde{\xi}\right\|_{v, p, 1} & \\
& \leq C_{K}\left|\pi_{v ;-} \tilde{\xi}(\infty)\right| \leq C_{K}^{\prime}\left(|\tilde{\xi}(\infty)|+\left|\pi_{v ;+} \tilde{\xi}(\infty)\right|\right) \\
& \leq C_{K}^{\prime \prime}\left(|\xi(\infty)|+\left(\|\widetilde{J}-J\|_{C^{1}}+\|\zeta\|_{v, p, 1}\right)\|\tilde{\xi}\|_{v, p, 1}\right) \quad \forall \xi \in \operatorname{ker} D_{\widetilde{J}, \tilde{u}} \\
\Longrightarrow & \|\widetilde{\xi}\|_{v, p, 1} \leq C_{K}\left\|\tilde{\pi}_{v ;-} \pi_{v ;-} \tilde{\xi}\right\|_{v, p, 1} \quad \forall \xi \in \operatorname{ker} \mathfrak{D}_{\widetilde{J}, \widetilde{u}}^{\infty},
\end{aligned}
$$

if $\delta_{K}$ is sufficiently small. Thus,

$$
\operatorname{dim} \operatorname{ker} \mathfrak{D}_{\widetilde{J}, \widetilde{u}}^{\infty} \leq \operatorname{dim} \widetilde{\Gamma}_{-}(v)=\operatorname{ind} \mathfrak{D}_{J, b}^{\infty}=\operatorname{ind} \mathfrak{D}_{\widetilde{J}, \tilde{u}}^{\infty},
$$

and the operator $\mathfrak{D}_{\widetilde{J}, \widetilde{u}}^{\infty}$ is surjective.

\subsection{Some power-series expansions}

In Section 2.5 of [13] we describe the behavior of all derivatives of rational $J_{0}-$ holomorphic maps into $\mathbb{P}^{n}$ near each stratum $\mathcal{U}_{\mathcal{T}}\left(\mathbb{P}^{n} ; J_{0}\right)$ by making use of special properties of the standard complex structure $J_{0}$ on $\mathbb{P}^{n}$. In this subsection, we obtain analogous estimates for modified derivatives of $J$-holomorphic maps into $X$ for an arbitrary genus-zero $A$-regular almost complex structure $J$; see Lemma 3.5. We use these estimates a number of times in the rest of the paper.

If $b=\left(\Sigma_{b}, u_{b}\right)$ is as element of $\tilde{\mathcal{U}}_{\mathcal{T}}^{(0)}(X ; J)$ as in the previous subsection, the tangent bundle $T_{b} \widetilde{\mathcal{U}}_{\mathcal{T}}^{(0)}(X ; J)$ of $\tilde{\mathcal{U}}_{\mathcal{T}}^{(0)}(X ; J)$ at $b$ consists of the pairs $(\underline{w}, \xi)$, where $\xi \in$ $\operatorname{ker} D_{J, b}$ and $\underline{w} \in \mathbb{C}^{\widehat{I}}$ encode the change in $u_{b}$ and in the position of the nodes on $\Sigma_{b}$, respectively, that satisfy a certain balancing condition; see Section 2.5 in [16]. We denote by $\widetilde{T}_{b} \widetilde{\mathcal{U}}_{\mathcal{T}}^{(0)}(X ; J)$ the subspace of the tuples $(0, \xi)$ of $T_{b} \widetilde{\mathcal{U}}_{\mathcal{T}}^{(0)}(X ; J)$. In particular,

$\widetilde{T}_{b} \tilde{\mathcal{U}}_{\mathcal{T}}^{(0)}(X ; J) \subset \Gamma_{-}(b) \equiv\left\{\left(\xi_{h}\right)_{h \in I} \in \bigoplus_{h \in I} \operatorname{ker} D_{J, u_{b, h}}: \xi_{h}(\infty)=\xi_{\iota_{h}}\left(x_{h}(b)\right) \forall h \in \hat{I}\right\}$,

where $u_{b, h}=\left.u_{b}\right|_{\Sigma_{b, h}}$. If $i \in \chi(\mathcal{T})$, where $\chi(\mathcal{T})$ is as in (2-3), the image of the projection map

$$
\begin{gathered}
\left\{\left(\xi_{h}\right)_{h \in I} \in \widetilde{T}_{b} \tilde{\mathcal{U}}_{\mathcal{T}}^{(0)}(X ; J): \xi_{i}(\hat{0}, \infty)=0\right\} \longrightarrow\left\{\zeta \in \operatorname{ker} D_{J, u_{b, i}}: \zeta(\widehat{0}, \infty)=0\right\}, \\
\xi=\left.\left(\xi_{h}\right)_{h \in I} \longrightarrow \xi\right|_{\Sigma_{b, i}}=\xi_{i},
\end{gathered}
$$

has real codimension two. Its complement corresponds to the infinitesimal translations in $\mathbb{C} \subset \Sigma_{b, i}$. Thus, if $J$ satisfies the regularity conditions (a) and (b-i) of Definition 1.4 
and $\hat{0}$ is the minimal element of $I$, for all $i \in \chi(\mathcal{T})$ the map

$\mathfrak{D}_{J, u ; i}: \widetilde{\Gamma}_{-}(b) \equiv\left\{\xi \in \widetilde{T}_{b} \tilde{\mathcal{U}}_{\mathcal{T}}^{(0)}(X ; J): \xi(\widehat{0}, \infty)=0\right\} \longrightarrow T_{\mathrm{ev}_{0}(b)} X, \mathfrak{D}_{J, u ; i}(\xi)=\nabla_{e_{\infty}}^{J} \xi_{i}$, is surjective.

Lemma 3.3 If $(X, \omega, J), A$, and $\mathcal{T}$ are as in Lemma 3.1, for every precompact open subset $K$ of $\mathcal{U}_{\mathcal{T}}(X ; J)$, there exist $\delta_{K}, \epsilon_{K}, C_{K} \in \mathbb{R}^{+}$and an open neighborhood $U_{K} \subset U_{\mathcal{T}}$ of $K$ in $\mathfrak{X}_{0, M}(X, A)$ with the following properties:

(1) Requirements (1) and (2) of Corollary 3.2 are satisfied.

(2) If $\widetilde{J}$ is an almost complex structure on $X$ such that $\|\widetilde{J}-J\|_{C^{1}} \leq \delta_{K}$,

(2a) for all $v=\left.(b, v) \in \widetilde{\mathcal{F}}_{\delta_{K}}^{\varnothing}\right|_{\tilde{K}^{(0)}}$, the equation

$$
\bar{\partial}_{\widetilde{J}} \exp _{u_{v}} \zeta=0, \quad \zeta \in \Gamma_{+}(v),\|\zeta\|_{v, p, 1}<\epsilon_{K},
$$

has a unique solution $\zeta_{\widetilde{J}, v}$;

(2b) the map

$\tilde{\phi}_{\tilde{J}}:\left.\widetilde{\mathcal{F}}_{\delta_{K}}^{\varnothing}\right|_{\tilde{K}^{(0)}} \longrightarrow \mathfrak{M}_{0,\{0\} \sqcup M}^{0}(X, A ; \widetilde{J}) \cap U_{K}, \quad v \longrightarrow\left[\exp _{b(v)} \zeta_{\widetilde{J}_{, v}}\right]$,

is smooth;

(2c) for all $v=\left.(b, v) \in \widetilde{\mathcal{F} \mathcal{T}}_{\delta_{K}}^{\varnothing}\right|_{\widetilde{K}^{(0)}}, \operatorname{ev}_{0}\left(\tilde{\phi}_{\widetilde{J}}(v)\right)=\operatorname{ev}_{0}(b)$;

(2d) for all $v=(b, v) \in \widetilde{\mathcal{F}}_{\delta_{K}}^{\varnothing_{K}} \mid \widetilde{K}^{(0)}$,

$$
\left\|\zeta_{\widetilde{J}, v}\right\|_{v, p, 1},\left\|\nabla^{\mathcal{T}} \zeta_{\tilde{J}, v}\right\|_{v, p, 1} \leq C_{K}\left(\|\widetilde{J}-J\|_{C^{1}}+|v|^{1 / p}\right),
$$

where $\nabla^{\mathcal{T}} \zeta_{\widetilde{J}, v}$ denotes the differential of the bundle map $v \longrightarrow \zeta_{\widetilde{J}, v}$ along $\widetilde{\Gamma}_{-}(b)$ with respect to a connection in the bundle $\Gamma((\cdot, v))$ over $\tilde{\mathcal{U}}_{\mathcal{T}}^{(0)}(X ; J)$.

Remark Let $\varphi: \tilde{\Gamma}_{-}(\cdot) \longrightarrow \tilde{\mathcal{U}}_{\mathcal{T}}^{(0)}(X ; J)$ be a smooth map such that

$$
\left.d \varphi\right|_{(b, 0)}: \tilde{\Gamma}_{-}(b) \longrightarrow T_{b} \tilde{\mathcal{U}}_{\mathcal{T}}^{(0)}(X ; J)
$$

is the inclusion map for all $b \in \tilde{\mathcal{U}}_{\mathcal{T}}^{(0)}(X ; J)$ and

$$
\Sigma_{\varphi(\varsigma)}=\Sigma_{b} \quad \text { and } \quad \operatorname{ev}_{0}(\varphi(\varsigma))=\operatorname{ev}_{0}(b) \quad \forall b \in \tilde{\mathcal{U}}_{\mathcal{T}}^{(0)}(X ; J), \varsigma \in \tilde{\Gamma}_{-}(b) .
$$

Let

$$
\tilde{\varphi}: \pi_{\tilde{\Gamma}_{-}(\cdot)}^{*} \Gamma((\cdot, v))=\pi_{\tilde{\Gamma}_{-}(\cdot)}^{*} \Gamma\left(\Sigma_{(\cdot, v)} ; u_{(\cdot, v)}^{*} T X\right) \longrightarrow \Gamma((\cdot, v))
$$


be a lift of $\varphi$ to a vector-bundle homomorphism that restricts to the identity over $\tilde{\mathcal{U}}_{\mathcal{T}}^{(0)}(X ; J)$. For example, we can take $\tilde{\varphi}$ to be given by

$$
\{\widetilde{\varphi}(\varsigma ; \xi)\}(z)=\Pi_{\zeta\left(q_{(b, v)}(z)\right)} \xi(z) \quad \forall z \in \Sigma_{(b, v)}=\Sigma_{(\varphi(\varsigma), v)}
$$

if

$$
\varsigma \in \widetilde{\Gamma}_{-}(b) \text { and } \varphi(\varsigma)=\left(\Sigma_{b}, \exp _{u_{b}} \zeta(z)\right)
$$

where $\Pi_{\zeta\left(q_{(b, v)}(z)\right)} \xi(z)$ is the parallel transport of $\xi(z)$ along the geodesic

$$
\gamma_{\zeta(z)}:[0,1] \longrightarrow X, \quad \tau \longrightarrow \exp _{u_{(b, v)}(z)} \tau \zeta\left(q_{(b, v)}(z)\right) .
$$

We can then define $\nabla^{\mathcal{T}} \zeta_{\widetilde{J},(\cdot, v)}: \widetilde{\Gamma}_{-}(\cdot) \longrightarrow \Gamma((\cdot, v))$ by

$$
\left.\left\{\nabla_{\zeta}^{\mathcal{T}} \zeta_{\tilde{J}, .}\right\}\right|_{(b, v)}=\lim _{t \longrightarrow 0} \frac{\zeta_{\tilde{J},(\varphi(t \varsigma), v)}-\tilde{\varphi}\left(\zeta_{\tilde{J},(b, v)}\right)}{t} \in \Gamma((b, v))
$$

for $X \in \widetilde{\Gamma}_{-}(b)$. Finally, a choice of metric on $\tilde{\mathcal{U}}_{\mathcal{T}}^{(0)}(X ; J)$ determines $\left\|\nabla^{\mathcal{T}} \zeta_{\widetilde{J}, v}\right\|_{v, p, 1}$.

Claim (2a) of Lemma 3.3 and the first bound in (2d) follow immediately from (1)

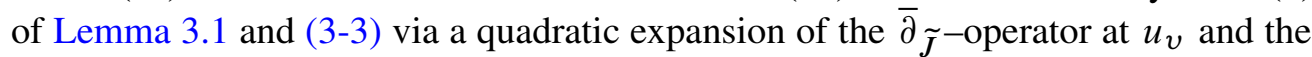
Contraction Principle; see [16, Section 3.6]. Claim (2c) is a consequence of (3-2). The smoothness of the map $\widetilde{\phi}_{\widetilde{J}}$ follows from the smooth dependence of solutions of the equation in (2a) on the parameters. The second bound in (2d) is obtained from the uniform behavior of these parameters; see [16, Section 3.4].

If $b \in \tilde{\mathcal{U}}_{\mathcal{T}}^{(0)}(X ; J)$ is as before, the domain $\Sigma_{b}$ of $b$ has the form

$$
\Sigma_{b}=\left(\bigsqcup_{i \in I}\{i\} \times S^{2}\right) / \sim, \quad \text { where } \quad(h, \infty) \sim\left(\iota_{h}, x_{h}(b)\right) \forall h \in \widehat{I},
$$

and $x_{h}(b) \in S^{2}-\{\infty\}$. The basic gluing map $q_{v}: \Sigma_{v} \longrightarrow \Sigma_{b}$ used in this paper is a homeomorphism outside of at most $|\widehat{I}|$ circles in $\Sigma_{v}$ and is holomorphic outside of the annuli

$$
\tilde{A}_{v, h}^{ \pm} \equiv q_{v}^{-1}\left(A_{b, h}^{ \pm}\left(\left|v_{h}\right|\right)\right)
$$

with $h \in \widehat{I}$, where

$$
\begin{aligned}
& A_{b, h}^{-}(\delta)=A_{h}^{-}(\delta)=\left\{(h, z) \in\{h\} \times S^{2}:|z| \geq \delta^{-1 / 2} / 2\right\}, \\
& A_{b, h}^{+}(\delta)=\left\{\left(\iota_{h}, z\right) \in\left\{\iota_{h}\right\} \times S^{2}:\left|z-x_{h}(b)\right| \leq 2 \delta^{1 / 2}\right\},
\end{aligned}
$$


for any $\delta \in \mathbb{R}^{+}$. For each $h \in \hat{I}, \tilde{A}_{v, h}^{+} \cup \widetilde{A}_{v, h}^{-}$is the thin neck of $\Sigma_{v}$ corresponding to smoothing the node of $\Sigma_{b}$ joining the spheres $\Sigma_{b, l_{h}}$ and $\Sigma_{b, h}$. If $\delta \in \mathbb{R}^{+}$, let

$$
\begin{gathered}
\partial^{-} A_{b, h}^{-}(\delta)=\left\{(h, z) \in\{h\} \times S^{2}:|z|=\delta^{-1 / 2} / 2\right\}, \\
\Sigma_{b}^{0}(\delta)=\left(\Sigma_{b}-\bigcup_{i \in \chi(\mathcal{T})} \bigcup_{h \geq i} \Sigma_{b, h}\right) \cup \bigcup_{i \in \chi(\mathcal{T})} A_{b, i}^{-}(\delta), \\
\tilde{A}_{v, h}^{ \pm}(\delta)=q_{v}^{-1}\left(A_{b, h}^{ \pm}(\delta)\right) \subset \Sigma_{v}, \quad \partial^{-} \tilde{A}_{v, h}^{-}(\delta)=q_{v}^{-1}\left(\partial^{-} A_{b, h}^{-}(\delta)\right), \\
\Sigma_{v}^{0}(\delta)=q_{v}^{-1}\left(\Sigma_{b}^{0}(\delta)\right) .
\end{gathered}
$$

In the case of Figure $7, \Sigma_{v}^{0}(\delta)$ consists of the two nonshaded components, with the node joining them turned into a thin neck, the three thin necks corresponding to the nodes attaching the bubbles $h_{1}, h_{4}$, and $h_{5}$, and small annuli extending from each of these three necks into the interior of the corresponding bubble, provided $|v|<\delta$. If $v=(b, v)$, with $v \in \mathbb{C}^{* \widehat{I}}$, the complement of $\Sigma_{v}^{0}(\delta)$ is the union of $|\chi(\mathcal{T})|$ disks that support nearly all of the map $u_{v} \equiv u_{b} \circ q_{v}$.

Lemma 3.4 If $(X, \omega, J), A$, and $\mathcal{T}$ are as in Lemma 3.1, for every precompact open subset $K$ of $\mathcal{U}_{\mathcal{T}}(X ; J)$, there exist $\delta_{K}, \epsilon_{K}, C_{K} \in \mathbb{R}^{+}$and an open neighborhood $U_{K} \subset U_{\mathcal{T}}$ of $K$ in $\mathfrak{X}_{0, M}(X, A)$ with the following properties:

(1) Requirements (1) and (2) of Corollary 3.2 are satisfied.

(2) If $\widetilde{J}$ is an almost complex structure on $X$ such that $\|\widetilde{J}-J\|_{C^{1}} \leq \delta_{K}$, there exist a smooth map

$$
\tilde{\phi}_{\widetilde{J}}:\left.\widetilde{\mathcal{F}}_{\delta_{K}}^{\varnothing}\right|_{\widetilde{K}^{(0)}} \longrightarrow \mathfrak{M}_{0,\{0\} \sqcup M}^{0}(X, A ; \widetilde{J}) \cap U_{K}
$$

such that the requirements (2a)-(2d) of Lemma 3.3 are satisfied. Furthermore, for every $b \in \widetilde{K}^{(0)}$ and $v=\left.(b, v) \in \widetilde{\mathcal{F}}_{\delta_{K}}^{\varnothing}\right|_{\tilde{K}^{(0)}}$, there exist

$$
\begin{array}{ll}
\Phi_{b} \in L_{1}^{p}\left(\Sigma_{b}^{0}\left(\delta_{K}\right) ; \operatorname{End}\left(\mathrm{ev}_{0}^{*} T X\right)\right), & \vartheta_{b} \in \operatorname{Hol}_{J}\left(\Sigma_{b}^{0}\left(\delta_{K}\right) ; \mathrm{ev}_{0}^{*} T X\right), \\
\Phi_{\widetilde{J}, v} \in L_{1}^{p}\left(\Sigma_{v}^{0}\left(\delta_{K}\right) ; \operatorname{End}\left(\mathrm{ev}_{0}^{*} T X\right)\right), & \vartheta_{\widetilde{J}, v} \in \operatorname{Hol}_{J}\left(\Sigma_{v}^{0}\left(\delta_{K}\right) ; \mathrm{ev}_{0}^{*} T X\right),
\end{array}
$$

such that

(2a) the maps $b \longrightarrow\left(\Phi_{b}, \vartheta_{b}\right)$ and $v \longrightarrow\left(\Phi_{\widetilde{J}, v}, \vartheta_{\widetilde{J}, v}\right)$ are smooth;

(2b) for all $b \in \widetilde{K}^{(0)}$,

$$
\begin{gathered}
\exp _{\operatorname{ev}_{0}(b)}\left(\Phi_{b}(z) \vartheta_{b}(z)\right)=u_{b}(z) \quad \forall z \in \Sigma_{b}^{0}\left(\delta_{K}\right), \\
\left.\Phi_{b}\right|_{\Sigma_{b}^{0}(0)}=\mathrm{Id} \quad \text { and } \quad\left\|\Phi_{b}-\mathrm{Id}\right\|_{b, p, 1},\left\|\nabla^{\mathcal{T}}\left(\Phi_{b}-\mathrm{Id}\right)\right\|_{b, p, 1} \leq \frac{1}{2} ;
\end{gathered}
$$




$$
\begin{aligned}
& \text { (2c) for all } v=\left.(b, v) \in \widetilde{\mathcal{F}}_{\delta_{K}}^{\varnothing}\right|_{\tilde{K}^{(0)}}, \\
& \quad \exp _{\mathrm{ev}_{0}(b)}\left(\Phi_{\widetilde{J}, v}(z) \vartheta_{\widetilde{J}_{, v}}(z)\right)=\tilde{u}_{v}(z) \quad \forall z \in \Sigma_{v}^{0}\left(\delta_{K}\right), \\
& \left\|\Phi_{\widetilde{J}_{, v}}-\Phi_{b} \circ q_{v}\right\|_{v, p, 1},\left\|\nabla^{\mathcal{T}}\left(\Phi_{\widetilde{J}_{, v}}-\Phi_{b} \circ q_{v}\right)\right\|_{v, p, 1} \leq C_{K}\left(\|\widetilde{J}-J\|_{C^{1}}+|v|^{1 / p}\right), \\
& \text { if } \tilde{u}_{v}=\exp _{u_{v}} \zeta_{\tilde{J}_{, v}} .
\end{aligned}
$$

In the statement of this lemma, $\operatorname{Hol}_{J}\left(\Sigma_{b}^{0}\left(\delta_{K}\right) ; \mathrm{ev}_{0}^{*} T X\right)$ and $\operatorname{Hol}_{J}\left(\Sigma_{v}^{0}\left(\delta_{K}\right) ; \mathrm{ev}_{0}^{*} T X\right)$ denote the spaces of holomorphic maps from $\Sigma_{b}^{0}\left(\delta_{K}\right)$ and $\Sigma_{v}^{0}\left(\delta_{K}\right)$ into the complex vector space $\left(T_{\mathrm{ev}_{0}(b)} X, J\right)$. In brief, the substance of Lemma 3.4 is that a $J$-holomorphic map can be well approximated by a holomorphic map on a neighborhood of the primary marked point, or any other point, of the domain. Due to Lemma 3.3, Lemma 3.4 is essentially a parametrized version of Theorem 2.2 in [3], and only a couple of additional ingredients are needed. The crucial fact used in [3] is that the operator

$$
\begin{gathered}
\Xi: L_{1}^{p}\left(S^{2} ; \operatorname{End}_{\mathbb{C}}\left(\mathbb{C}^{n}\right)\right) \longrightarrow L^{p}\left(S^{2} ; \Lambda^{0,1} T^{*} S^{2} \otimes \operatorname{End}_{\mathbb{C}}\left(\mathbb{C}^{n}\right)\right) \oplus \operatorname{End}_{\mathbb{C}}\left(\mathbb{C}^{n}\right), \\
\Xi(\Theta)=(\bar{\partial} \Theta, \Theta(0)),
\end{gathered}
$$

is an isomorphism. The map $\Xi$ is still an isomorphism if $S^{2}$ is replaced by a tree of spheres $\Sigma$ and 0 by any point on $\Sigma$. Furthermore, if $y$ is a smooth point of $\Sigma$ for all sufficiently small smoothings $v$ of the nodes, the operators

$$
\begin{gathered}
\Xi_{v}: L_{1}^{p}\left(\Sigma_{v} ; \operatorname{End}_{\mathbb{C}}\left(\mathbb{C}^{n}\right)\right) \longrightarrow L^{p}\left(\Sigma_{v} ; \Lambda^{0,1} T^{*} \Sigma_{v} \otimes \operatorname{End}_{\mathbb{C}}\left(\mathbb{C}^{n}\right)\right) \oplus \operatorname{End}_{\mathbb{C}}\left(\mathbb{C}^{n}\right), \\
\Xi_{v}(\Theta)=(\bar{\partial} \Theta, \Theta(y)),
\end{gathered}
$$

are also isomorphisms. In addition, for some $C \in \mathbb{R}^{+}$and for all sufficiently small smoothings $v$,

$$
C^{-1}\left\|\Xi_{v} \Theta\right\|_{v, p} \leq\|\Theta\|_{v, p, 1} \leq C\left\|\Xi_{v} \Theta\right\|_{v, p} \quad \forall \Theta \in L_{1}^{p}\left(\Sigma_{v} ; \operatorname{End}_{\mathbb{C}}\left(\mathbb{C}^{n}\right)\right)
$$

If all components of $v$ are nonzero, $\Sigma_{v}$ is topologically a sphere, but should instead be viewed as a tree of spheres joined by thin necks. As before, we denote by $\|\cdot\|_{v, p, 1}$ and $\|\cdot\|_{v, p}$ the norms induced by the pregluing construction above. In particular, (3-10) can be viewed as a special case of (1) of Lemma 3.1. We need to use the norms $\|\cdot\|_{v, p, 1}$ and $\|\cdot\|_{v, p}$, since these are the norms used in Lemmas 3.1 and 3.3. Keeping track of all norms in the proof of Theorem 2.2 in [3], we see that the maps $\Phi_{b}, \vartheta_{b}$, $\Phi_{\widetilde{J}, v}$, and $\vartheta_{\widetilde{J}, v}$ satisfying (2b) and the first condition in (2c) exist, provided that $\delta_{K}$ is sufficiently small. The last two estimates in (2c) are obtained by an argument similar to Section 4.1 in [16]. 




$(\hat{0}, \infty)$

$$
\begin{aligned}
& \chi(\mathcal{T})=\left\{h_{1}, h_{4}, h_{5}\right\} \\
& \rho(v)=\left(v_{h_{1}}, v_{h_{3}} v_{h_{4}}, v_{h_{3}} v_{h_{5}}\right) \\
& x_{h_{5}}(v)=x_{h_{3}}(b)+v_{h_{3}} x_{h_{5}}(b)
\end{aligned}
$$

$$
\mathcal{D}_{\hat{0}}^{(1)} \tilde{b}_{\tilde{J}}(v) \cong \mathcal{D}_{J, h_{1}}^{(1)} v_{h_{1}}+\mathcal{D}_{J, h_{4}}^{(1)} v_{h_{3}} v_{h_{4}}+\mathcal{D}_{J, h_{5}}^{(1)} v_{h_{3}} v_{h_{5}}
$$

Figure 7: An example of the estimates of Lemma 3.5

Before we can state Lemma 3.5, we need to introduce additional notation. For each $v=(b, v)$, where $b \in \widetilde{\mathcal{U}}_{\mathcal{T}}^{(0)}(X ; J)$ and $v \in \mathbb{C}^{\hat{I}}$, and $i \in \chi(\mathcal{T})$, let

$$
\rho_{i}(v)=\prod_{\hat{0}<h \leq i} v_{h} \in \mathbb{C}, \quad \rho_{i}(v)=\left(b, \rho_{i}(v)\right) ; \quad x_{i}(v)=\sum_{\substack{\hat{0}<i^{\prime} \leq i\\}}\left(x_{i^{\prime}}(b) \prod_{\substack{\hat{0}<h<i^{\prime}}} v_{h}\right) \in \mathbb{C},
$$

where $x_{i}(b)$ is as in (3-9); see Figure 7. If $K$ and $\vartheta_{b}$ are as in Lemma 3.4, $b=$ $\left(\Sigma_{b}, u_{b}\right) \in \widetilde{K}^{(0)}, v \in \mathbb{C}, i \in \chi(\mathcal{T})$, and $r \in \mathbb{Z}^{+}$, we put

$$
\mathcal{D}_{i}^{(r)} b=\left.\frac{1}{r !} \frac{d^{r}}{d w_{i}^{r}} \vartheta_{b, i}\left(w_{i}\right)\right|_{w_{i}=0} \in T_{\mathrm{ev}_{0}(b)} X \quad \text { and } \quad \mathcal{D}_{J, i}^{(r)}(b, v)=v \cdot{ }_{J} \mathcal{D}_{i}^{(r)} b,
$$

where $\vartheta_{b, i}=\left.\vartheta_{b}\right|_{\Sigma_{b, i}}, \cdot J$ is the scalar multiplication in $(T X, J)$, and $w_{i}$ is the standard holomorphic coordinate centered at the point $\infty$ in $\Sigma_{b, i}=S^{2}$. If $\left.v \in \widetilde{\mathcal{F}}_{\delta_{K}}^{\varnothing}\right|_{\tilde{K}^{(0)}}$, we similarly set

$$
\tilde{b}_{\widetilde{J}}(v)=\left(\Sigma_{v}, \tilde{u}_{v}\right), \quad \mathcal{D}_{\widehat{0}}^{(r)} \tilde{b}_{\widetilde{J}}(v)=\left.\frac{1}{r !} \frac{d^{r}}{d w^{r}} \vartheta_{\widetilde{J}, v}(w)\right|_{w=0} \in T_{\operatorname{ev}_{0}(b)} X,
$$

where $w$ is the standard holomorphic coordinate centered at the point $\infty$ in $\Sigma_{v} \approx S^{2}$. The value of $\mathcal{D}_{i}^{(r)} b$ depends on the choice of $\vartheta_{b}$ in Lemma 3.4, which can be uniquely prescribed by the choice of $\delta_{K} \in \mathbb{R}^{+}$. Alternatively, one can replace small positive numbers $\delta_{K}$ dependent on compact subsets $K$ of $\mathcal{U}_{\mathcal{T}}(X ; J)$ by a small function $\delta: \mathcal{U}_{\mathcal{T}}(X ; J) \longrightarrow \mathbb{R}^{+}$, which can be used to choose a holomorphic map

$$
\vartheta_{b}: \Sigma_{b}^{0}(\delta(b)) \longrightarrow\left(T_{\mathrm{ev}_{0}(b)} X, J\right)
$$

for each $J$-holomorphic stable map $b \in \tilde{\mathcal{U}}_{\mathcal{T}}^{(0)}(X ; J)$. Of course, the definition of $\mathcal{D}_{\hat{0}}^{(r)} \widetilde{b}_{\widetilde{J}}(v)$ depends on even more choices, including those involved in the gluing construction of Lemma 3.3. 
Lemma 3.5 If $(X, \omega, J), A$, and $\mathcal{T}$ are as in Lemma 3.1, for every precompact open subset $K$ of $\mathcal{U}_{\mathcal{T}}(X ; J)$, there exist $\delta_{K}, \epsilon_{K}, C_{K} \in \mathbb{R}^{+}$and an open neighborhood $U_{K} \subset U_{\mathcal{T}}$ of $K$ in $\mathfrak{X}_{0, M}(X, A)$ with the following properties:

(1) Requirements (1) and (2) of Corollary 3.2 are satisfied.

(2) If $\widetilde{J}$ is an almost complex structure on $X$ such that $\|\widetilde{J}-J\|_{C^{1}} \leq \delta_{K}$, there exist $\tilde{\phi}_{\widetilde{J}}, \Phi_{b}$ and $\vartheta_{b}$ for each $b \in \widetilde{K}^{(0)}$, and $\Phi_{\widetilde{J}, v}$ and $\vartheta_{\widetilde{J}_{, v}}$ for each $\left.v \in \widetilde{\mathcal{F}}_{\delta_{K}}^{\varnothing}\right|_{\widetilde{K}^{(0)}}$, such that the requirements (2a)-(2d) of Lemma 3.3 and (2a)-(2c) of Lemma 3.4 are satisfied. Furthermore, for each $k \in \mathbb{Z}^{+}$and $i \in \chi(\mathcal{T})$ there exists a smooth map

$$
\varepsilon_{\widetilde{J}, i}^{(k)}:\left.\widetilde{\mathcal{F}}_{\delta_{K}}^{\varnothing}\right|_{\tilde{K}^{(0)}} \longrightarrow \mathrm{ev}_{0}^{*} T X
$$

such that

(2a) for all $r \in \mathbb{Z}^{+}$and $v=\left.(b, v) \in \widetilde{\mathcal{F}}_{\delta_{K}}^{\varnothing}\right|_{\tilde{K}^{(0)}}$,

$\mathcal{D}_{\widehat{0}}^{(r)} \tilde{b}_{J}(v)=\sum_{k=1}^{k=r}\left(\begin{array}{l}r-1 \\ k-1\end{array}\right) \sum_{i \in \chi(\mathcal{T})} x_{i}^{r-k}(v)\left\{\mathcal{D}_{J, i}^{(k)}+\varepsilon_{\widetilde{J}, i}^{(k)}(v)\right\} \rho_{i}^{k}(v) \in T_{\mathrm{ev}_{0}(b)} X ;$

(2b) for all $k \in \mathbb{Z}^{+}, i \in \chi(\mathcal{T})$, and $v=\left.(b, v) \in \widetilde{\mathcal{F}}_{\delta_{K}}^{\varnothing}\right|_{\tilde{K}^{(0)}}$,

$$
\left|\varepsilon_{\widetilde{J}, i}^{(k)}\right|_{v},\left|\nabla^{\mathcal{T}} \varepsilon_{\widetilde{J}, i}^{(k)}\right| v \leq C_{K} \delta_{K}^{-k / 2}\left(\|\widetilde{J}-J\|_{C^{1}}+|v|^{1 / p}\right) .
$$

Proof (1) We apply, with some modifications, the argument for the analytic estimates of Theorem 2.8 in [13] to holomorphic functions $\vartheta_{b}$ and $\vartheta_{\widetilde{J}, v}$, instead of the functions $u_{b}$ and $\tilde{u}_{v}$ which are $J$-holomorphic and $\widetilde{J}$-holomorphic in this case. We will use coordinates $z$ on $S^{2}-\{\infty\} \approx \mathbb{C}$ and $w=z^{-1}$ on $S^{2}-\{0\}$. Since $\vartheta_{\widetilde{J}, v}$ is holomorphic on $\Sigma_{v}^{0}\left(\delta_{K}\right)$,

$$
\begin{aligned}
& \mathcal{D}_{\hat{0}}^{(r)} \tilde{b}_{J}(v)=\left.\frac{1}{r !} \frac{\partial^{r}}{\partial w^{r}} \vartheta_{\widetilde{J}, v}(w)\right|_{w=0}=\frac{1}{2 \pi \mathfrak{i}} \oint_{\partial \Sigma_{v}^{0}\left(\delta_{K}\right)} \vartheta_{\widetilde{J}, v}(w) \frac{d w}{w^{r+1}} \\
&=\frac{1}{2 \pi \mathfrak{i}} \sum_{i \in \chi(\mathcal{T})} \oint_{\partial^{-} \tilde{A}_{v, i}^{-}\left(\delta_{K}\right)} \vartheta_{\widetilde{J}, v}(w) \frac{d w}{w^{r+1}} \\
&=-\frac{1}{2 \pi \mathfrak{i}} \sum_{i \in \chi(\mathcal{T})} \oint_{\partial^{-11)}} \tilde{A}_{v, i}^{-}\left(\delta_{K}\right) \\
& \Phi_{\tilde{J}, v}^{-1}(z)\left(\Phi_{b} \vartheta_{b} \circ q_{v}(z)+\tilde{\zeta}_{\tilde{J}, v}(z)\right) z^{r-1} d z,
\end{aligned}
$$

where $\widetilde{\zeta}_{\tilde{J}_{, v}} \in C^{\infty}\left(\Sigma_{v}^{0}\left(\delta_{K}\right) ; T_{\mathrm{ev}_{0}(b)} X\right)$ is defined by

$$
\exp _{\operatorname{ev}_{0}(b)}\left(\exp _{\operatorname{ev}_{0}(b)}^{-1} u_{v}(z)+\tilde{\zeta}_{\tilde{J}_{, v}}\right)=\tilde{u}_{v}(z) \equiv \exp _{u_{v}(z)} \zeta_{\tilde{J}_{, v}}(z)
$$


(2) In order to estimate each integral on the last line of (3-11), we expand $z^{r-1}$ around $x_{i}(v)$, the center of the circle $\partial^{-} \widetilde{A}_{v, i}^{-}\left(\delta_{K}\right)$, as a polynomial in $\widetilde{z}_{i}=z-x_{i}(v)$ :

$$
\oint_{\partial-\tilde{A}_{v, i}^{-}\left(\delta_{K}\right)} \Phi_{\widetilde{J}, v}^{-1}(z)\left(\Phi_{b} \vartheta_{b} \circ q_{v}(z)+\widetilde{\zeta}_{\widetilde{J}, v}(z)\right) z^{r-1} d z
$$

$$
=\sum_{k=1}^{k=r}\left(\begin{array}{l}
r-1 \\
k-1
\end{array}\right) x_{i}^{r-k}(v) \oint_{\partial-\tilde{A}_{v, i}^{-}\left(\delta_{K}\right)} \Phi_{\widetilde{J}, v}^{-1}\left(\widetilde{z}_{i}\right)\left(\Phi_{b} \vartheta_{b} \circ q_{v}\left(\widetilde{z}_{i}\right)+\widetilde{\zeta}_{\tilde{J}_{, v}}\left(\widetilde{z}_{i}\right)\right) \widetilde{z}_{i}^{k-1} d \widetilde{z}_{i} .
$$

By construction, $z_{i}=q_{v}\left(\widetilde{z}_{i}\right)=\rho_{i}^{-1}(v) \widetilde{z}_{i}$ near $\partial^{-} \widetilde{A}_{v, i}^{-}\left(\delta_{K}\right)$, if $z_{i}$ is the standard holomorphic coordinate on $\Sigma_{v, i}-\{\infty\}=\{i\} \times\left(S^{2}-\{\infty\}\right)$ and $|v|<\delta_{K}$. Since $\left.\vartheta_{b, i} \equiv \vartheta_{b}\right|_{\Sigma_{b, i}}$ is $J_{\mathrm{ev}_{0}(b)}$-holomorphic,

$$
\begin{aligned}
\oint_{\partial-\tilde{A}_{v, i}^{-}\left(\delta_{K}\right)} \vartheta_{b}\left(q_{v}\left(\tilde{z}_{i}\right)\right) \tilde{z}_{i}^{k-1} d \widetilde{z}_{i} & =\rho_{i}^{k}(v) \oint_{\partial^{-} A_{i}^{-}\left(\delta_{K}\right)} \vartheta_{b, i}\left(z_{i}\right) z_{i}^{k-1} d z_{i} \\
& =-\rho_{i}^{k}(v) \oint_{\partial-A_{i}^{-}\left(\delta_{K}\right)} \vartheta_{b, i}\left(w_{i}\right) \frac{d w_{i}}{w_{i}^{k+1}} \\
& =-\left.\rho_{i}^{k}(v) \frac{2 \pi \mathfrak{i}}{k !} \frac{\partial^{k}}{\partial w_{i}^{k}} \vartheta_{b, i}\left(w_{i}\right)\right|_{w_{i}=0} \\
& =-2 \pi \mathfrak{i} \mathcal{D}_{J, i}^{(k)} \rho_{i}^{k}(v),
\end{aligned}
$$

where $w_{i}=z_{i}^{-1}$ is the standard holomorphic coordinate on $\{i\} \times\left(S^{2}-\{0\}\right)$. Similarly,

$$
\begin{array}{r}
\oint_{\partial^{-} \tilde{A}_{v, i}^{-}\left(\delta_{K}\right)}\left(\left\{\Phi_{\tilde{J}, v}^{-1}\left(\tilde{z}_{i}\right) \Phi_{b}\left(q_{v}\left(\tilde{z}_{i}\right)\right)-\operatorname{Id}\right\} \vartheta_{b}\left(q_{v}\left(\tilde{z}_{i}\right)\right)+\Phi_{\widetilde{J}, v}^{-1}\left(\tilde{z}_{i}\right) \tilde{\zeta}_{\tilde{J}, v}\left(\tilde{z}_{i}\right)\right) \tilde{z}_{i}^{k-1} d \widetilde{z}_{i} \\
=-2 \pi \mathfrak{i} \varepsilon_{\widetilde{J}, i}^{(k)}(v) \rho_{i}^{k}(v),
\end{array}
$$

where

$$
\begin{aligned}
\varepsilon_{\widetilde{J}, i}^{(k)}(v)=\frac{1}{2 \pi \mathfrak{i}} \oint_{\partial-A_{i}^{-}\left(\delta_{K}\right)}\left(\left\{\Phi_{\widetilde{J}, v}^{-1}\left(w_{i}\right) \Phi_{b}\left(q_{v}\left(w_{i}\right)\right)-\operatorname{Id}\right\} \vartheta_{b}\left(q_{v}\left(w_{i}\right)\right)\right. & \\
& \left.+\Phi_{\widetilde{J}, v}^{-1}\left(w_{i}\right) \widetilde{\zeta} \widetilde{J}_{, v}\left(w_{i}\right)\right) \frac{d w_{i}}{w_{i}^{k+1}}
\end{aligned}
$$

The expansion in (2a) of the lemma follows immediately from (3-11)-(3-14). By definition, $\partial^{-} A_{i}^{-}\left(\delta_{K}\right)$ is a circle of radius $2 \delta_{K}^{1 / 2}$ around the south pole in the sphere $\Sigma_{b, i}$. Thus, part (2b) of the lemma follows from (3-15), along with (3-8) and the bounds in $(2 b)$ and $(2 c)$ of Lemma 3.4. 


\subsection{The genus-one regularity properties $(b-i)$ and $(b-i i)$}

In this subsection, we show that if $J$ is an almost complex structure on $X$ that satisfies the regularity conditions (a) and (b-i) of Definition 1.4, then so does every almost complex structure $\tilde{J}$ on $X$ which is sufficiently close to $J$. This claim follows from Corollary 3.6 and the compactness of the moduli space $\overline{\mathfrak{M}}_{0,1}(X, A ; J)$. We also show that if $J$ is an almost complex structure on $X$ that satisfies the regularity conditions (a), (b-i), and (b-ii) of Definition 1.4, then so does every nearby almost complex structure $\widetilde{J}$ on $X$. This conclusion is immediate from Corollary 3.7 and the compactness of the moduli space $\overline{\mathfrak{M}}_{0,2}(X, A ; J)$.

If $\mathcal{T}$ is a bubble type as in Corollary 3.2 with $A_{\hat{0}}=0$, where $\hat{0}$ is the minimal element of $I$, the analogue of (3-6) does not hold for the map $\mathfrak{D}_{J, b}^{\infty, v}$ for any fixed nonzero vector $v$ tangent to $\mathbb{P}^{1}$ at $\infty$. The reason is that $\mathfrak{D}_{J, b}^{\infty, v}$ is the zero homomorphism on ker $\mathfrak{D}_{J, b}^{\infty}$, since the map $u_{b}$ is constant on the component $\Sigma_{b, \hat{0}}$ of the domain $\Sigma_{b}$ of $b$ which contains the marked point $\infty$. In particular, $\mathfrak{D}_{\widetilde{J}, \tilde{u}}^{\infty, v}$ need not be surjective for a smooth map $\tilde{u}: \mathbb{P}^{1} \longrightarrow X$ which is arbitrary close to the moduli space $\overline{\mathfrak{M}}_{0,1}(X, A ; J)$. Thus, a different approach is required to understand the behavior of the regularity condition (b-i) of Definition 1.4 near $\mathcal{U}_{\mathcal{T}}(X ; J)$.

Claim (c) of Theorem 1.6 can alternatively be viewed as a statement concerning the behavior of the first derivatives $\left.d u\right|_{\infty}$ of $J$-holomorphic maps. Lemma 3.5 describes the behavior of modified first and higher-order derivatives of $\widetilde{J}$-holomorphic maps near a stratum $\mathcal{U}_{\mathcal{T}}(X ; J)$ with $\mathcal{T}$ as in the previous paragraph. We use the estimate for the higher-order derivatives to describe the behavior of the regularity condition (b-ii) of Definition 1.4 near $\mathcal{U}_{\mathcal{T}}(X ; J)$.

Corollary 3.6 Suppose $(X, \omega, J), A \neq 0$, and $\mathcal{T}$ are as in Lemma 3.1 and $M=\varnothing$. If the almost complex structure $J$ satisfies the regularity conditions (a) and (b-i) of Definition 1.4, for every precompact open subset $K$ of $\mathcal{U}_{\mathcal{T}}(X ; J)$, there exist $\delta_{K}, C_{K} \in \mathbb{R}^{+}$and an open neighborhood $U_{K} \subset U_{\mathcal{T}}$ of $K$ in $\mathfrak{X}_{0, \varnothing}(X, A)$ with the following properties:

(1) Requirements (1) and (2) of Lemma 3.1 are satisfied.

(2) If $\widetilde{J}$ is an almost complex structure on $X$ such that $\|\widetilde{J}-J\|_{C^{1}}<\delta_{K}$ and $\left[\mathbb{P}^{1}, \widetilde{u}\right] \in \mathfrak{M}_{0,\{0\}}^{0}(X, A ; \widetilde{J}) \cap U_{K}$, the operators $D_{\widetilde{J}, \tilde{u}}, \mathfrak{D}_{\widetilde{J}, \tilde{u}}^{\infty}$, and $\mathfrak{D}_{\widetilde{J}, \tilde{u}}^{\infty, e_{\infty}}$ are surjective.

Proof (1) By Corollary 3.2, it remains to show that the operator $\mathfrak{D}_{\widetilde{J}}^{\infty, e_{\infty}}$ is surjective. If $\mathcal{T}=(\varnothing, I ;, \underline{A})$ with $A_{\hat{0}} \neq 0$, the argument used twice in the proof of Corollary 3.2 
can be repeated once more to show that the operator $\mathfrak{D}_{\widetilde{J}, \tilde{u}}^{\infty, e_{\infty}}$ is also surjective for any smooth map $\tilde{u}$ sufficiently close to $K$. Thus, we will assume that $A_{\hat{0}}=0$.

(2) Let $q$ be any point in $X$. By (a) of Definition 1.4,

$$
\tilde{\mathcal{U}}_{\mathcal{T}}^{(0)}(q ; J) \equiv\left\{b \in \tilde{\mathcal{U}}_{\mathcal{T}}^{(0)}(X ; J): \mathrm{ev}_{0}(b)=q\right\}
$$

is a smooth submanifold of $\tilde{\mathcal{U}}_{\mathcal{T}}^{(0)}(X ; J)$. By Corollary 3.2, $\mathfrak{M}_{0,\{0\}}^{0}(X, A ; \widetilde{J})$ is a smooth orbifold, while

$$
\mathfrak{M}_{0,\{0\}}^{0}(q ; \widetilde{J}) \equiv\left\{b \in \mathfrak{M}_{0,\{0\}}^{0}(X, A ; \widetilde{J}): \operatorname{ev}_{0}(b)=q\right\}
$$

is a smooth suborbifold of $\mathfrak{M}_{0,\{0\}}^{0}(X, A ; \widetilde{J})$. By Lemma 3.3, every element of $\mathfrak{M}_{0,\{0\}}^{0}(q ; \widetilde{J}) \cap U_{K}$ has a representative of the form $\left(\mathbb{P}^{1}, \tilde{u}\right)$, where

$$
\tilde{u}=\exp _{u_{v}} \zeta_{\tilde{J}, v}, \quad v=\left.(b, v) \in \widetilde{\mathcal{F}} \mathcal{T}_{\delta_{K}}^{\varnothing}\right|_{\tilde{K}^{(0)}},
$$

and $\zeta_{\widetilde{J}, v}$ is as in (2a) of Lemma 3.3. By (2c) of Lemma 3.3,

$$
\begin{aligned}
\left.\frac{d}{d t} \exp _{u_{(\varphi(t \xi), v)}} \zeta_{\tilde{J},(\varphi(t \xi), v)}\right|_{t=0} & \in \operatorname{ker} \mathfrak{D}_{\widetilde{J}, \tilde{u}}^{\infty} \subset \operatorname{ker} D_{\widetilde{J}, \widetilde{u}} \\
& \forall \xi \in \widetilde{T}_{b} \widetilde{\mathcal{U}}_{\mathcal{T}}^{(0)}(X ; J) \cap T_{b} \widetilde{\mathcal{U}}_{\mathcal{T}}^{(0)}(q ; J)=\widetilde{\Gamma}_{-}(b),
\end{aligned}
$$

where $\varphi$ is the map defined in the remark following Lemma 3.3. We will show that the map

$$
\tilde{\Gamma}_{-}(b) \longrightarrow T_{q} X,\left.\quad \xi \longrightarrow \frac{d}{d t}\left\{\left.d\left\{\exp _{u_{(\varphi(t \xi), v)}} \zeta_{\tilde{J},(\varphi(t \xi), v)}\right\}\right|_{\infty} e_{\infty}\right\}\right|_{t=0},
$$

is surjective. Along with (3-16), this claim implies Corollary 3.6.

(3) Let $\Phi_{b}, \vartheta_{b}, \Phi_{\widetilde{J}, v}$, and $\vartheta_{\widetilde{J}, v}$ be as in Lemma 3.4. Since for all $v^{\prime}=\left(b^{\prime}, v\right) \in$ $\left.\widetilde{\mathcal{F} \mathcal{T}_{\delta_{K}}^{\varnothing}}\right|_{\widetilde{K}^{(0)}}, \Phi_{\widetilde{J}_{,} v^{\prime}}$ is an $L_{1}^{p}$-map on $\Sigma_{v^{\prime}}^{0}\left(\delta_{K}\right) \subset \mathbb{P}^{1}$, while the $J_{\mathrm{ev}_{0}\left(b^{\prime}\right)}$-holomorphic map $\vartheta_{\widetilde{J}, v^{\prime}}$ vanishes at $\infty \in \Sigma_{v^{\prime}}^{0}\left(\delta_{K}\right)$,

$$
\begin{aligned}
\left.d\left\{\exp _{u_{v^{\prime}}} \zeta_{\widetilde{J}, v^{\prime}}\right\}\right|_{\infty} e_{\infty} & =\left.d\left\{\Phi_{\widetilde{J}, v^{\prime}} \vartheta_{\widetilde{J}, v^{\prime}}\right\}\right|_{\infty} e_{\infty} \\
& =\left\{\Phi_{\widetilde{J}, v^{\prime}}(\infty)\right\}\left(\left.d \vartheta_{\widetilde{J}, v^{\prime}}\right|_{\infty} e_{\infty}\right)
\end{aligned}
$$

Thus, by the $r=1$ case of (2a) of Lemma 3.5,

$$
\begin{aligned}
\left.d\left\{\exp _{u_{v^{\prime}}} \zeta{\widetilde{J}, v^{\prime}}\right\}\right|_{\infty} e_{\infty} & =\left\{\Phi_{\widetilde{J}, v^{\prime}}(\infty)\right\} \mathcal{D}_{\widehat{0}}^{(1)} \tilde{b}_{\widetilde{J}}\left(v^{\prime}\right) \\
& =\left\{\Phi_{\widetilde{J}, v^{\prime}}(\infty)\right\} \sum_{i \in \chi(\mathcal{T})}\left\{\left(\mathcal{D}_{i}^{(1)} b^{\prime}\right)+\varepsilon_{\widetilde{J}, i}^{(1)}\left(v^{\prime}\right)\right\} \rho_{i}\left(v^{\prime}\right)
\end{aligned}
$$


Replacing $b^{\prime}$ with $\varphi(t \xi)$ in (3-18) and differentiating at $t=0$, we obtain

$$
\begin{aligned}
& \left.\frac{d}{d t}\left\{\left.d\left\{\exp _{u_{(\varphi(t), v)}} \zeta_{\widetilde{J},(\varphi(t \xi), v)}\right\}\right|_{\infty} e_{\infty}\right\}\right|_{t=0} \\
& =\left\{\nabla_{\xi}^{\mathcal{T}} \Phi_{\widetilde{J}, v}(\infty)\right\} \sum_{i \in \chi(\mathcal{T})}\left\{\left(\mathcal{D}_{i}^{(1)} b\right)+\varepsilon \varepsilon_{\widetilde{J}, i}^{(1)}(v)\right\} \rho_{i}(v) \\
& \quad+\left\{\Phi_{\widetilde{J}, v}(\infty)\right\} \sum_{i \in \chi(\mathcal{T})}\left\{\nabla_{\xi}^{\mathcal{T}}\left(\mathcal{D}_{i}^{(1)} b\right)+\nabla_{\xi}^{\mathcal{T}} \varepsilon_{\widetilde{J}, i}^{(1)}(v)\right\} \rho_{i}(v)
\end{aligned}
$$

By (2b) and (2c) of Lemma 3.4 and (2b) of Lemma 3.5,

$$
\begin{aligned}
& \left|\Phi_{\widetilde{J}, v}^{-1}(\infty)-\operatorname{Id}\right|,\left|\varepsilon_{\widetilde{J}, h}^{(1)}(v)\right| \leq C_{K}\left(\|\tilde{J}-J\|_{C^{1}}+|v|^{1 / p}\right), \\
& \left|\nabla_{\xi}^{\mathcal{T}} \Phi_{\widetilde{J}, v}(\infty)\right|,\left|\nabla_{\xi}^{\mathcal{T}} \varepsilon_{\widetilde{J}, h}^{(1)}(v)\right| \leq C_{K}\left(\|\widetilde{J}-J\|_{C^{1}}+|v|^{1 / p}\right)\|\xi\|_{b, p, 1} .
\end{aligned}
$$

On the other hand, since $\vartheta_{b}(\infty)=0$,

$$
\begin{aligned}
\nabla_{\xi}^{\mathcal{T}}\left(\mathcal{D}_{i}^{(1)} b\right) & =\left.\Phi_{b}(\infty) \frac{d}{d t} \nabla_{\xi}^{\mathcal{T}}\left(\left.d u_{\varphi(t \xi), i}\right|_{\infty} e_{\infty}\right)\right|_{t=0}=\left.\frac{d}{d t}\left(\left.d u_{\varphi(t \xi), i}\right|_{\infty} e_{\infty}\right)\right|_{t=0} \\
& =\nabla_{e_{\infty}}^{J}\left(\left.\xi\right|_{\Sigma_{b, i}}\right) \equiv \mathfrak{D}_{J, b ; i} \xi \quad \forall \xi \in \widetilde{\Gamma}_{-}(b) .
\end{aligned}
$$

By (a) and (b-i) of Definition 1.4, the map

$$
\mathfrak{D}_{J, b ; i}: \tilde{\Gamma}_{-}(b) \longrightarrow T_{\mathrm{ev}_{0}(b)} X
$$

is surjective for all $i \in \chi(\mathcal{T})$; see the paragraph preceding Lemma 3.3. Since $\rho_{i}(v) \in \mathbb{C}^{*}$ for all $v=\left.(b, v) \in \widehat{\mathcal{F}} \mathcal{T}_{\delta_{K}}^{\varnothing}\right|_{\tilde{K}^{(0)}}$ and $i \in \chi(\mathcal{T})$, it follows from (3-19)-(3-21) that the map in (3-17) is also surjective, provided $\delta_{K}$ is sufficiently small.

Remark At the end of the argument above, we use the fact that $\chi(\mathcal{T}) \neq \varnothing$. This is the case if and only if $A \neq 0$.

Corollary 3.7 Suppose $(X, \omega, J), A \neq 0$, and $\mathcal{T}$ are as in Lemma 3.1 and $M=\{1\}$. If the almost complex structure $J$ satisfies the regularity conditions (a), (b-i), and (b-ii) of Definition 1.4, for every precompact open subset $K$ of $\mathcal{U}_{\mathcal{T}}(X ; J)$, there exist $\delta_{K}, C_{K} \in \mathbb{R}^{+}$and an open neighborhood $U_{K} \subset U_{\mathcal{T}}$ of $K$ in $\mathfrak{X}_{0,\{1\}}(X, A)$ with the following properties:

(1) Requirements (1) and (2) of Lemma 3.1 are satisfied.

(2) If $\widetilde{J}$ is an almost complex structure on $X$ such that $\|\widetilde{J}-J\|_{C^{1}}<\delta_{K}$ and

$$
\left[\mathbb{P}^{1}, y_{1}, \tilde{u}\right] \in \mathfrak{M}_{0,\{0,1\}}^{0}(X, A ; \widetilde{J}) \cap U_{K},
$$

the operators $D_{\widetilde{J}, \tilde{u}}, \mathfrak{D}_{\widetilde{J}, \tilde{u}}^{\infty}$, and $\mathfrak{D}_{\widetilde{J}, \tilde{u}}^{\infty, y_{1}}$ are surjective. 
Proof (1) By Corollary 3.2, it remains to show that the operator $\mathfrak{D}_{\widetilde{J}, \widetilde{u}}^{\infty, y_{1}}$ is surjective. If

$$
\mathcal{T}=(\{1\}, I ; j, \underline{A})
$$

is a bubble type such that $A_{i} \neq 0$ for some $i \leq j_{1}$, the argument used in the proof of Corollary 3.2 can be repeated once more to show that the operator $\mathfrak{D}_{\widetilde{J}, \tilde{u}}^{\infty}, y_{1}$ is also surjective for any smooth map $\left(y_{1}, \tilde{u}\right)$, with two marked points, sufficiently close to $K$. Thus, we will assume that $A_{i}=0$ for all $i \leq j_{1}$. In this case, $\operatorname{ev}_{0}(b)=\mathrm{ev}_{1}(b)$ for all $b \in \mathcal{U}_{\mathcal{T}}(X ; J)$, as there are no nonghost components between the marked points $(\widehat{0}, \infty)$ and $y_{1}$. In the case of Figure 7 , this means that $j_{1} \in\left\{\hat{0}, h_{3}\right\}$, ie $y_{1}$ lies on one of the nonshaded bubbles.

(2) For any point $q \in X$, let $\tilde{\mathcal{U}}_{\mathcal{T}}^{(0)}(q ; J)$ and $\mathfrak{M}_{0,\{0,1\}}^{0}(q ; \widetilde{J})$ be defined as in (2) of the proof of Corollary 3.6. By Lemma 3.3, every element of $\mathfrak{M}_{0,\{0,1\}}^{0}(q ; \widetilde{J}) \cap U_{K}$ has a representative of the form $\left(\mathbb{P}^{1}, \tilde{y}_{1}, \tilde{u}\right)$, where

$$
\begin{gathered}
\tilde{u}=\exp _{u_{v}} \zeta_{\tilde{J}, v}, \quad \tilde{y}_{1}=y_{1}(v)=\sum_{\hat{0}<i<j_{1}} x_{i}(b) \prod_{\hat{0}<h<i} v_{h}+y_{1} \prod_{\hat{0}<h \leq j_{1}} v_{h} \in \mathbb{C}, \\
v=\left.(b, v) \in \widetilde{\mathcal{F}}_{\delta_{K}}^{\varnothing}\right|_{\tilde{K}^{(0)}},
\end{gathered}
$$

and $\zeta_{\widetilde{J}, v}$ is as in (2a) of Lemma 3.3. We will show that the map

$$
\tilde{\Gamma}_{-}(b) \longrightarrow T_{q} X,\left.\quad \xi \longrightarrow \frac{d}{d t}\left\{\exp _{q} \zeta_{\widetilde{J},(\varphi(t \xi), v)}\left(\tilde{y}_{1}\right)\right\}\right|_{t=0},
$$

is surjective. Note that $q=u_{(\varphi(t \xi), v)}(\widehat{0}, \infty)$. Along with (3-16), this claim implies Corollary 3.7.

(3) Let $\Phi_{b}, \vartheta_{b}, \Phi_{\widetilde{J}, v}$, and $\vartheta_{\widetilde{J}, v}$ be as in Lemma 3.4. Since $\vartheta_{\widetilde{J}, v}$ is a $J_{\mathrm{ev}_{0}(b)}-$ holomorphic map on $\Sigma_{v}^{0}\left(\delta_{K}\right)$, vanishing at $\infty$, and $\tilde{y}_{1} \in \Sigma_{v}^{0}\left(\delta_{K}\right)$, for all $v^{\prime}=$ $\left.\left(b^{\prime}, v\right) \in \widetilde{\mathcal{F}}_{\delta_{K}}^{\varnothing}\right|_{\tilde{K}^{(0)}}$,

$$
\begin{aligned}
\vartheta_{\widetilde{J}, v^{\prime}}\left(\tilde{y}_{1}\right) & =\left.\sum_{r=1}^{\infty} \frac{1}{r !} \frac{d^{r}}{d w^{r}} \vartheta_{\widetilde{J}, v^{\prime}}(w)\right|_{w=0} \cdot \tilde{y}_{1}^{-r}=\sum_{r=1}^{\infty} \mathcal{D}_{\widehat{0}}^{(r)} \tilde{b}_{\widetilde{J}}\left(v^{\prime}\right) \cdot \tilde{y}_{1}^{-r} \\
& =\sum_{k=1}^{\infty} \sum_{i \in \chi(\mathcal{T})}\left(\tilde{y}_{1}-x_{i}\left(v^{\prime}\right)\right)^{-k}\left\{\mathcal{D}_{J, i}^{(k)}+\varepsilon_{\widetilde{J}, i}^{(k)}\left(v^{\prime}\right)\right\} \rho_{i}^{k}\left(v^{\prime}\right) \in T_{\mathrm{ev}_{0}\left(b^{\prime}\right)} X,
\end{aligned}
$$

by (2a) of Lemma 3.5; see the proof of Lemma 4.2 in [17] for more details. For each $i \in \chi(\mathcal{T})$, we denote by $h(i)$ the largest element of $\hat{I}$ such that $h(i) \leq i$ and $\iota_{h(i)} \leq j_{1}$. We set

$$
x_{h ; 1}\left(b^{\prime}\right)=x_{h(i)}\left(b^{\prime}\right) \quad \text { and } \quad y_{1 ; h}\left(b^{\prime}\right)= \begin{cases}y_{1}\left(b^{\prime}\right), & \text { if } \iota_{h(i)}=j_{1} \\ x_{\widetilde{h}}\left(b^{\prime}\right), & \text { if } \widetilde{h} \leq j_{1} \text { and } \iota_{\widetilde{h}}=\iota_{h(i)} .\end{cases}
$$


If $v^{\prime}=\left(b^{\prime}, v\right)$, we put

$$
\begin{gathered}
\rho_{i ; 1}\left(v^{\prime}\right)=\prod_{h(i) \leq h \leq i} v_{h}, \quad x_{i ; 1}\left(v^{\prime}\right)=\sum_{h(i) \leq i^{\prime} \leq i}\left(x_{i^{\prime}}\left(b^{\prime}\right) \prod_{h(i) \leq h<i^{\prime}} v_{h}\right) \in \mathbb{C}, \\
y_{1 ; i}\left(v^{\prime}\right)=\sum_{\iota_{h(i)}<i^{\prime}<j_{1}} x_{i^{\prime}}\left(b^{\prime}\right) \prod_{\iota_{h(i)}<h<i^{\prime}} v_{h}+y_{1} \prod_{\iota_{h(i)}<h \leq j_{1}} v_{h} \in \mathbb{C} .
\end{gathered}
$$

It is straightforward to see from the definitions that

$$
\left(y_{1}\left(v^{\prime}\right)-x_{i}\left(v^{\prime}\right)\right)^{-1} \rho_{i}\left(v^{\prime}\right)=\left(y_{1 ; i}\left(v^{\prime}\right)-x_{i ; 1}\left(v^{\prime}\right)\right)^{-1} \rho_{i ; 1}\left(v^{\prime}\right) .
$$

By (3-23) and (3-24),

$$
\begin{aligned}
\vartheta_{\widetilde{J}, v^{\prime}}\left(\tilde{y}_{1}\right)= & \sum_{i \in \chi(\mathcal{T})}\left(y_{1 ; i}\left(b^{\prime}\right)-x_{i ; 1}\left(b^{\prime}\right)\right)^{-1}\left\{\left(\mathcal{D}_{J, i}^{(1)} b^{\prime}\right)+\varepsilon_{\widetilde{J}, i}^{(0)}\left(v^{\prime}\right)\right\} \rho_{i ; 1}(v) \in T_{\mathrm{ev}_{0}\left(b^{\prime}\right)} X, \\
& \left|\varepsilon_{\widetilde{J}, i}^{(0)}\left(v^{\prime}\right)\right|,\left|\nabla^{\mathcal{T}} \varepsilon_{\widetilde{J}, i}^{(0)}\left(v^{\prime}\right)\right| \leq C_{K}\left(\|\widetilde{J}-J\|_{C^{1}}+\left|v^{\prime}\right|^{1 / p}\right) .
\end{aligned}
$$

Finally, for all $\xi \in \widetilde{T}_{b} \tilde{\mathcal{U}}_{\mathcal{T}}^{(0)}(X ; J)$,

$$
\begin{gathered}
\tilde{y}_{1}(\varphi(t \xi), v)=\tilde{y}_{1}(b, v), \\
\tilde{y}_{1 ; i}(\varphi(t \xi))=\tilde{y}_{1 ; i}(b), \quad x_{i ; 1}(\varphi(t \xi))=x_{i ; 1}(b) \quad \forall i \in \chi(\mathcal{T}) .
\end{gathered}
$$

The surjectivity of the map in (3-22) follows from (3-25) and (3-26), along with (2) of Lemma 3.4, by the same argument as in (3) of the proof of Corollary 3.6.

Corollaries 3.6 and 3.7 complete the proof of the parts of Theorem 1.6 that concern genus-zero stable maps. However, this is a convenient point to deduce a few more conclusions from Lemma 3.5. We use Corollary 3.8 in the next three sections. Suppose $\mathcal{T}=(M, I ; j, \underline{A})$ is any bubble type and $v=(b, v) \in \widetilde{\mathcal{F} \mathcal{T}}$ is sufficiently small. With notation as in the proof of Corollary 3.6 and

$$
\tilde{u}_{v}=\exp _{b, u_{v}} \zeta_{\widetilde{J}, v}, \quad \tilde{\Gamma}(v ; \widetilde{J})=L_{1}^{p}\left(\Sigma_{v} ; \tilde{u}_{v}^{*} T X\right),
$$

we define the homomorphism

$$
\widetilde{R}_{\widetilde{J}, v}: \widetilde{\Gamma}_{-}(b) \longrightarrow \operatorname{ker} \mathfrak{D}_{\widetilde{J}, \widetilde{u}_{v}}^{\infty} \subset \widetilde{\Gamma}(v ; \widetilde{J}) \text { by } \widetilde{R}_{\widetilde{J}, v} \xi=\left.\frac{d}{d t} \exp _{u_{(\varphi(t \xi), v)}} \zeta_{\widetilde{J},(\varphi(t \xi), v)}\right|_{t=0}
$$

Denote the image of $\widetilde{R}_{\widetilde{J}_{, v}}$ by $\widetilde{\Gamma}_{-}(v ; \widetilde{J})$. Let

$$
\begin{aligned}
A_{v, \hat{0}}^{-}(\delta) & =\left\{(\hat{0}, z) \in\{\hat{0}\} \times S^{2}:|z| \geq \delta^{-1 / 2} / 2\right\} \subset \Sigma_{v}, \\
\partial^{-} A_{v, \hat{0}}^{-}(\delta) & \left.=\{\widehat{0}, z) \in\{\widehat{0}\} \times S^{2}:|z|=\delta^{-1 / 2} / 2\right\} \subset \Sigma_{v} .
\end{aligned}
$$


Corollary 3.8 If $(X, \omega, J), A$, and $\mathcal{T}$ are as in Lemma 3.1, for every precompact open subset $K$ of $\mathcal{U}_{\mathcal{T}}(X ; J)$, there exist $\delta_{K}, \epsilon_{K}, C_{K} \in \mathbb{R}^{+}$and an open neighborhood $U_{K} \subset U_{\mathcal{T}}$ of $K$ in $\mathfrak{X}_{0, M}(X, A)$ with the following properties:

(1) Requirements (1) and (2) of Lemma 3.1 are satisfied.

(2) If $\widetilde{J}$ is an almost complex structure on $X$ such that $\|\widetilde{J}-J\|_{C^{1}} \leq \delta_{K}$, there exists $\zeta_{\tilde{J}_{,},} \in \Gamma_{+}(v)$ for each $v=\left.(b, v) \in \widetilde{\mathcal{F}}_{\delta_{K}}^{\varnothing}\right|_{\tilde{K}^{(0)}}$ such that

(2a) requirements (2a)-(2d) of Lemma 3.3 are satisfied;

(2b) for all $v=\left.(b, v) \in \widetilde{\mathcal{F}}_{\delta_{K}}^{\varnothing}\right|_{\widetilde{K}^{(0)}}$ and $\delta \leq 4 \delta_{K}$, if $\tilde{u}_{v}=\exp _{u_{v}} \zeta_{\widetilde{J}, v}$,

$$
\left\|\left.d \tilde{u}_{v}\right|_{A_{v, \hat{0}}^{-}(\delta)}\right\|_{v, p} \leq C_{K} \delta^{1 / p} \sum_{i \in \chi(\mathcal{T})}\left|\rho_{i}(v)\right| ;
$$

(2c) for all $v=\left.(b, v) \in \widetilde{\mathcal{F}}_{\delta_{K}}^{\varnothing}\right|_{\tilde{K}^{(0)}}$ and $\delta \leq 4 \delta_{K}$, if $\exp _{\mathrm{ev}_{0}(b)} \tilde{f}_{v}(z)=\tilde{u}_{v}(z)$ for $z \in A_{v, \hat{0}}^{-}\left(\delta_{K}\right)$, then

$$
\left|\tilde{f}_{v}(w)\right| \leq C_{K}|w| \sum_{i \in \chi(\mathcal{T})}\left|\rho_{i}(v)\right| \quad \forall w \in A_{v, \hat{0}^{-}}^{-}\left(\delta_{K}\right),
$$

$$
\begin{aligned}
& \mid \oint_{\partial-A_{v, \hat{0}}^{-}(\delta)} \tilde{f}_{v}(w) \frac{d w}{w^{2}}-2 \pi \mathfrak{i} \sum_{i \in \chi(\mathcal{T})} \mathcal{D}_{J, i} \rho_{i}(v) \mid \\
& \leq C_{K}\left(\|\widetilde{J}-J\|_{C^{1}}+|v|^{1 / p}+\delta^{(p-2) / p}\right) \sum_{i \in \chi(\mathcal{T})}\left|\rho_{i}(v)\right|,
\end{aligned}
$$

where $w$ is the standard holomorphic coordinate on the complement of $(0,0,1)$ in $S^{2}$;

(2d) for all $v=\left.(b, v) \in \widetilde{\mathcal{F}}_{\delta_{K}}^{\varnothing}\right|_{\widetilde{K}^{(0)}}, \xi \in \widetilde{\Gamma}_{-}(b)$, and $\delta \in\left(0,4 \delta_{K}\right)$,

$$
\left\|\widetilde{R}_{\widetilde{J}, v} \xi\right\|_{v, p, 1} \leq C_{K}\|\xi\|_{b, p, 1},
$$

$\left|\oint_{\partial^{-} A_{v, \hat{0}}^{-}(\delta)}\left\{R_{\widetilde{J}, v} \xi\right\}(w) \frac{d w}{w^{2}}-2 \pi \mathfrak{i} \sum_{i \in \chi(\mathcal{T})} \rho_{i}(v) \mathfrak{D}_{J, b ; i} \xi\right|$
$\leq C_{K}\left(\|\widetilde{J}-J\|_{C^{1}}+|v|^{1 / p}+\delta^{(p-2) / p}\right) \sum_{i \in \chi(\mathcal{T})}\left|\rho_{i}(v)\right| \cdot\|\xi\|_{v, p, 1} ;$

(2e) for all $v=\left.(b, v) \in \widetilde{\mathcal{F}}_{\delta_{K}}^{\varnothing}\right|_{\tilde{K}^{(0)}}, \xi \in \widetilde{\Gamma}_{-}(v ; \widetilde{J})$, and $\delta \leq 4 \delta_{K}$,

$$
\begin{aligned}
& |\xi|_{w} \leq C_{K}|w| \sum_{i \in \chi(\mathcal{T})}\left|\rho_{i}(v)\right| \cdot\|\xi\|_{v, p, 1} \quad \forall w \in A_{v, \hat{0}}^{-}(\delta), \\
& \left\|\left.\nabla \xi\right|_{A_{v, \hat{0}}^{-}(\delta)}\right\|_{v, p} \leq C_{K} \delta^{1 / p} \sum_{i \in \chi(\mathcal{T})}\left|\rho_{i}(v)\right| \cdot\|\xi\|_{v, p, 1},
\end{aligned}
$$

where $w$ is as in (2c). 
The first part of the proof of Corollary 3.2 shows that

$$
C_{K}^{-1}\|\xi\|_{b, p, 1} \leq\left\|\widetilde{R}_{\widetilde{J}_{, v}} \xi\right\|_{v, p, 1} \leq C_{K}\|\xi\|_{b, p, 1} \quad \forall \xi \in \widetilde{\Gamma}_{-}(b) .
$$

In fact, $C_{K}$ can be required to be arbitrary close to one in this case. By the proof of (3-25),

$$
\begin{aligned}
\tilde{f}_{v}(w) & =\Phi_{\widetilde{J}, v}(w) \vartheta_{\widetilde{J}, v}(w) \\
& =\Phi_{\widetilde{J}, v}(w) \cdot \sum_{i \in \chi(\mathcal{T})} w\left\{\left(\mathcal{D}_{J, i}^{(1)} b\right)+\varepsilon_{\widetilde{J}, i}^{(0)}(v)\right\} \rho_{i}(v)+\varepsilon(v, w)
\end{aligned}
$$

for all $w \in A_{v, \hat{0}}^{-}\left(\delta_{K}\right)$, where

$$
|\varepsilon(v, w)| \leq C_{K}|w|^{2} \sum_{i \in \chi(\mathcal{T})}\left|\rho_{i}(v)\right| \quad \text { and } \quad\left|\partial_{w} \varepsilon(v, w)\right| \leq C_{K}|w| \sum_{i \in \chi(\mathcal{T})}\left|\rho_{i}(v)\right| .
$$

Both estimates in (2c) are immediate from (3-27), (2b) and (2c) of Lemma 3.4, and Holder's inequality, since

$$
\left(\mathcal{D}_{i}^{(1)} b\right) \cdot \rho_{i}(v)=\mathcal{D}_{J, i} \rho_{i}(v)
$$

Differentiating (3-27) with respect to $w$ and integrating, we obtain (2b). The first bound in (2e) is obtained by differentiating (3-27) with respect to $\xi$, as in (3) of the proof of Corollary 3.6. The second estimate in (2e) follows by differentiating the resulting expression with respect to $w$ and integrating. Finally, in the remaining statement of (2d), each element $\xi(w)$ of $T_{\widetilde{u}_{v}(w)} X$ is identified with its preimage in $T_{\mathrm{ev}_{0}(b)} X$ under the parallel transport along the geodesics. This estimate follows by differentiating (3-27) with respect to $\xi$. Due to the first bound in (2e), the parallel transport and the geodesics can be defined either with respect to the $J$-compatible connection $\nabla^{J}$ or with the respect to the $\widetilde{J}$-compatible connection

$$
\nabla^{\widetilde{J}}=\frac{1}{2}\left(\nabla^{J}-\tilde{J} \nabla^{J} \widetilde{J}\right)
$$

in the bundle $T X \longrightarrow X$.

\section{Genus-one gluing procedures}

\subsection{A one-step gluing construction}

Our next goal is to show that the regularity condition (c) of Definition 1.4 is wellbehaved under small perturbations of the almost complex structure $J$. Corollaries 4.2 and 4.5 , along with the compactness of the moduli space $\overline{\mathfrak{M}}_{1}(X, J ; A)$, show that this 
is indeed the case. They conclude the proof of the $g=1$ case of the first claim in Theorem 1.6.

We denote by $\mathfrak{X}_{1, M}(X, A)$ the space of equivalence classes of all smooth maps into $X$ from genus-one Riemann surfaces with marked points indexed by the set $M$ in the homology class $A$ and by $\mathfrak{X}_{1, M}^{0}(X, A)$ the subset of $\mathfrak{X}_{1, M}(X, A)$ consisting of maps with smooth domains, ie smooth tori in this case. Similarly to the previous section, we need to describe smooth maps $\tilde{u}: \Sigma \longrightarrow X$ in $\mathfrak{X}_{1, \varnothing}(X, A)$ that lie close to each stratum $\mathcal{U}_{\mathcal{T}}(X ; J)$ of the moduli space $\overline{\mathfrak{M}}_{1, \varnothing}(X, J ; A)$. If $\mathcal{U}_{\mathcal{T}}(X ; J)$ is contained in $\mathfrak{M}_{1, \varnothing}^{\{0\}}(X, A ; J)$, the surjectivity of the operator $D_{\widetilde{J}, \widetilde{u}}$ can be shown by an argument similar to the proof of Corollary 3.2. This case is handled in this subsection. We will assume that $J$ is an almost complex structure that satisfies the regularity conditions (a), (b-ii), and (c) of Definition 1.4.

Let $\mathcal{T}=(M, I, \aleph ; j, \underline{A})$ be a bubble type such $\sum_{i \in I} A_{i}=A$ and $A_{i} \neq 0$ for some minimal element of $I$. We proceed similarly to Section 3.1. For each sufficiently small element $v=(b, v)$ of $\widetilde{\mathcal{F}}^{\varnothing}$, let

$$
b(v)=\left(\Sigma_{v}, j_{v}, u_{v}\right), \quad \text { where } \quad u_{v}=u_{b} \circ q_{v},
$$

be the corresponding approximately holomorphic stable map. Since the stable map $u_{b}$ is not constant on the principal component, the linearization $D_{J, b}$ of the $\bar{\partial}_{J}$-operator at $b$ is surjective by (a), (b-ii), and (c) of Definition 1.4. Thus, if $v$ is sufficiently small, the linearization

$$
D_{J, v}: \Gamma(v) \equiv L_{1}^{p}\left(\Sigma_{v} ; u_{v}^{*} T X\right) \longrightarrow \Gamma^{0,1}(v ; J) \equiv L^{p}\left(\Sigma_{v} ; \Lambda_{J, j_{v}}^{0,1} T^{*} \Sigma_{v} \otimes u_{v}^{*} T X\right),
$$

of the $\bar{\partial}_{J}$-operator at $b(v)$, defined via the connection $\nabla^{J}$, is also surjective. In particular, we can obtain an orthogonal decomposition

$$
\Gamma(v)=\Gamma_{-}(v) \oplus \Gamma_{+}(v)
$$

such that the linear operator $D_{J, v}: \Gamma_{+}(v) \longrightarrow \Gamma^{0,1}(v ; J)$ is an isomorphism, while $\Gamma_{-}(v)$ is close to $\Gamma_{-}(b) \equiv \operatorname{ker} D_{J, b}$. The relevant facts concerning the objects described in this paragraph are summarized in Lemma 4.1 below.

Remark 1 The focus of the pregluing construction described in [16] is attaching bubble trees of spheres to a fixed Riemann surface $\Sigma$. The present situation is of course different. However, the main ingredient in the pregluing construction is a smooth family of nearly holomorphic maps $q_{v}: \Sigma_{v} \longrightarrow \Sigma_{b}$, constructed using a metric on $\Sigma$. All other objects that appear in the above paragraph are essentially determined by the map $q_{v}$, and the homeomorphism type of $\Sigma_{b}$ plays little role. In the case $\boldsymbol{\kappa}=\varnothing$, ie the principal component $\Sigma_{b, \aleph}$ of the domain $\Sigma_{b}$ of every element $b$ of $\widetilde{\mathcal{U}}_{\mathcal{T}}^{(0)}(X ; J)$ 
is a smooth torus, we choose a family of Kahler metrics $\left\{g_{b_{0}}\right\}$ on the fibers of the semiuniversal bundle $\mathfrak{U}_{\mathcal{T}_{0}} \longrightarrow \mathcal{U}_{\mathcal{T}_{0}}(X ; J)$; see Section 2.2 for notation. If $v=(b, v)$ is a small element of $\widetilde{\mathcal{F} \mathcal{T}} \varnothing$ and $b_{0}=\tilde{\pi}_{P}(b)$, we construct the map $q_{v}: \Sigma_{v} \longrightarrow \Sigma_{b}$ as in Section 2.2 of [16], using the metric $g_{b_{0}}$ on $\Sigma_{b, \aleph}$.

Remark 2 In the case $\boldsymbol{\aleph} \neq \varnothing$, ie the principal component $\Sigma_{b, \boldsymbol{\aleph}}$ of any bubble map $b \in \tilde{\mathcal{U}}_{\mathcal{T}}^{(0)}(X ; J)$ is the circle of spheres $\Sigma_{\mathcal{\aleph}}$, we split the pregluing construction into two steps. The first step will correspond to gluing at the nodes $\aleph$ of the principal curve and the second to attaching the trees of spheres to the resulting elliptic curve. The bundle of gluing parameters $\mathcal{F} \mathcal{T}_{0}$ over $\mathcal{U}_{\mathcal{T}_{0}}(X ; J)$ has the form

$$
\mathcal{F} \mathcal{T}_{0}=\bigoplus_{(h, i) \in \mathbb{N}} L_{(h, i)}=\bigoplus_{(h, i) \in \mathbb{N}} L_{(h, i) ; 0} \otimes L_{(h, i) ; 1}
$$

for some line bundles $L_{(h, i) ; 0}, L_{(h, i) ; 1} \longrightarrow \mathcal{U}_{\mathcal{T}_{0}}(X ; J)$. In addition, there exist bundle maps

$$
\phi_{(h, i) ; 0}: L_{(h, i) ; 0} \longrightarrow \mathfrak{U}_{\mathcal{T}_{0}} \quad \text { and } \quad \phi_{(h, i) ; 1}: L_{(h, i) ; 1} \longrightarrow \mathfrak{U}_{\mathcal{T}_{0}}
$$

over $\mathcal{U}_{\mathcal{T}_{0}}(X ; J)$ such that for all $b_{0} \in \mathcal{U}_{\mathcal{T}_{0}}(X ; J)$

$$
\left.\phi_{(h, i) ; 0}\right|_{b_{0}}:\left.\left.L_{(h, i) ; 0}\right|_{b_{0}} \longrightarrow \Sigma_{b_{0}, h} \quad \phi_{(h, i) ; 1}\right|_{b_{0}}:\left.L_{(h, i) ; 1}\right|_{b_{0}} \longrightarrow \Sigma_{b_{0}, i}
$$

are biholomorphisms that take $\left(b_{0}, 0\right)$ to the node $(h, i)$ of $\Sigma_{b_{0}}$. Let

$$
\sigma_{\mathcal{T}_{0}}: \tilde{\mathfrak{U}}_{\mathcal{T}_{0}} \longrightarrow \mathcal{F} \mathcal{T}_{0 ; \delta}, \quad \text { where } \quad \delta \in C^{\infty}\left(\mathcal{U}_{\mathcal{T}_{0}}(X ; J) ; \mathbb{R}^{+}\right),
$$

be a semiuniversal family of deformations of the elliptic curve $\Sigma_{\aleph}$, along with the marked points indexed by $M_{0} \sqcup I_{1}$, where $M_{0}$ and $I_{1}$ are the sets of marked points lying on $\Sigma_{\aleph}$ and of first-level bubbles of the elements of $\tilde{\mathcal{U}}_{\mathcal{T}}^{(0)}(X ; J)$, respectively; see Section 2.2. In particular,

$$
\left.\tilde{\mathfrak{U}}_{\mathcal{T}_{0}}\right|_{\mathcal{U}_{\mathcal{T}_{0}}^{(0)}(X ; J)}=\mathfrak{U}_{\mathcal{T}_{0}} .
$$

A small neighborhood in $\widetilde{\mathfrak{U}}_{\mathcal{T}_{0}}$ of the section $\mathcal{Z}_{h, i}$ of $\sigma_{\mathcal{T}_{0}}$ over $\mathcal{U}_{\mathcal{T}_{0}}(X ; J)$ corresponding to the node $(h, i)$ of $\Sigma_{\aleph}$ can be identified with the set

$$
\begin{array}{r}
U_{(h, i)}=\left\{\left(b_{0}, v ; x, y\right): b_{0} \in \mathcal{U}_{\mathcal{T}_{0}}(X ; J) ;\left(b_{0}, v\right) \in \mathcal{F} \mathcal{T}_{0},\left(b_{0}, x\right) \in L_{(h, i) ; 0},\right. \\
\left.\left(b_{0}, y\right) \in L_{(h, i) ; 1} ;|v|,|x|,|y|<\delta(b) ; x y=v_{(h, i)}\right\},
\end{array}
$$

in such a way that $\sigma_{\mathcal{T}_{0}}\left(b_{0}, v ; x, y\right)=\left(b_{0}, v\right)$ and the fibers of $\sigma_{\mathcal{T}_{0}}$ are identified holomorphically. For each $\left(b_{0}, v\right) \in \mathcal{F} \mathcal{T}_{0 ; \delta}$, we set $\Sigma_{\left(b_{0}, v\right)}=\sigma_{\mathcal{T}_{0}}^{-1}\left(b_{0}, v\right)$. Let

$$
\tilde{\sigma}_{\mathcal{T}_{0}}: \tilde{\mathfrak{U}}_{\mathcal{T}_{0}} \longrightarrow \mathfrak{U}_{\mathcal{T}_{0}}=\sigma_{\mathcal{T}_{0}}^{-1}\left(\mathcal{U}_{\mathcal{T}_{0}}(X ; J)\right)
$$


be a smooth map such that $\tilde{\sigma}_{\mathcal{T}_{0}}\left(\Sigma_{\left(b_{0}, v\right)}\right) \subset \Sigma_{b_{0}},\left.\widetilde{\sigma}_{\mathcal{T}_{0}}\right|_{\Sigma_{\left(b_{0}, v\right)}}$ is holomorphic outside of the $|\boldsymbol{\aleph}|$ open sets $U_{(h, i)}$,

$\tilde{\sigma}_{\mathcal{T}_{0}}\left(b_{0}, v ; x, y\right)=\left\{\begin{array}{ll}\left(b_{0}, 0 ; \phi_{(h, i) ; 0}(x)\right), & \text { if }|x| \geq 2\left|v_{(h, i)}\right|^{1 / 2} ; \\ \left(b_{0}, 0 ; \phi_{(h, i) ; 1}(y)\right), & \text { if }|y| \geq 2\left|v_{(h, i)}\right|^{1 / 2} ;\end{array}\right.$ if $\left(b_{0}, v ; x, y\right) \in U_{(h, i)}$, and $\widetilde{\sigma}_{\mathcal{T}_{0}}\left(b_{0}, v ; x_{h}\left(b_{0}, v\right)\right)=\left(b_{0}, 0 ; x_{h}\left(b_{0}, 0\right)\right)$ for all $h \in M_{0} \sqcup I_{1}$ and for all $\left(b_{0}, v\right) \in$ $\mathcal{F} \mathcal{T}_{0 ; \delta}$, where $x_{h}\left(b_{0}, v\right)$ is the marked point indexed by $h$ on $\Sigma_{\left(b_{0}, v\right)}$ and $v_{(h, i)}$ is the $L_{(h, i)}$-component of $v$. The last condition can be used to define the points $x_{h}\left(b_{0}, v\right)$ for $v \neq 0$. Let $q_{\left(b_{0}, v\right) ; 0}$ denote the restriction of $\widetilde{\sigma}_{\mathcal{T}_{0}}$ to $\Sigma_{\left(b_{0}, v\right)}$. We choose a Riemannian metric on $\tilde{\mathfrak{U}}$ such that its restriction $g_{\left(b_{0}, v\right)}$ to each fiber $\Sigma_{\left(b_{0}, v\right)}$ of $\sigma_{\mathcal{T}_{0}}$ is Kahler. Along the way, we have made a number of choices. These choices will be fixed once and for all. If $v \in \widetilde{\mathcal{F} T}$, let

$$
v_{\boldsymbol{\aleph}}=\left(b, v_{\boldsymbol{\aleph}}\right), \quad \text { if } \quad v=\left(b, v_{\boldsymbol{\aleph}}, v_{\widehat{I}}\right),\left(b, v_{\boldsymbol{\aleph}}\right) \in \widetilde{\mathcal{F}_{\boldsymbol{\aleph}} \mathcal{T}},\left(b, v_{\widehat{I}}\right) \in \widetilde{\mathcal{F}_{0} \mathcal{T}} \oplus \widetilde{\mathcal{F}_{1} \mathcal{T}}
$$

see Section 2.2. If $v$ is sufficiently small, we denote by $\Sigma_{v_{\aleph}}$ the Riemann surface obtained from $\Sigma_{b}$ by replacing the circle of spheres $\Sigma_{b, \aleph}$ with $\left|M_{0}\right| \sqcup\left|I_{1}\right|$ marked points, which together we denote by $b_{0}$, by $\Sigma_{\left(b_{0}, v_{\aleph}\right)}$. Let

$$
q_{v_{\aleph}}: \Sigma_{v_{\aleph}} \longrightarrow \Sigma_{b}
$$

be the smooth map obtained by extending the map $q_{\left(b_{0}, v\right) ; 0}$ by identity to the rational components of $\Sigma_{b}$. We put

$$
v^{\aleph}=\left(b\left(v_{\aleph}\right),\left(q_{v_{\aleph}}^{*} v_{h}\right)_{h \in \hat{I}}\right),
$$

where

$$
b\left(v_{\aleph}\right)=\left(\Sigma_{v_{\aleph}}, u_{v_{\aleph}}\right), \quad u_{v_{\aleph}}=u_{b} \circ q_{v_{\aleph}}
$$

and

$$
q_{v_{\aleph}}^{*} v_{h}= \begin{cases}\left.d q_{v_{\aleph}}\right|_{x_{h}\left(v_{\aleph}\right)} ^{-1} v_{h} \in T_{x_{h}\left(v_{\aleph}\right)} \Sigma_{\left(b_{0}, v_{\aleph}\right)}, & \text { if } h \in I_{1} ; \\ v_{h} \in \mathbb{C}, & \text { if } h \in \hat{I}-I_{1} .\end{cases}
$$

Let $\left(\Sigma_{v}, j_{v}, g_{v}\right)$ be the Riemann surface obtained by attaching the bubble trees of spheres to the elliptic curve $\Sigma_{v_{\aleph}}$, using the gluing parameter $v^{\aleph}$ and the metric $g_{v_{\aleph}}$ on the principal component $\Sigma_{\left(b_{0}, v_{\aleph}\right)}$ of $\Sigma_{v_{\aleph}}$, via the procedure described in Section 2 and Section 3.3 of [16]. We take the key basic gluing map $q_{v}: \Sigma_{v} \longrightarrow \Sigma_{b}$ to be simply the composition $q_{v_{\aleph}} \circ q_{v^{*}}$.

Lemma 4.1 Suppose $(X, \omega, J)$ is a compact almost Kahler manifold, $A \in H_{2}(X ; \mathbb{Z})$, and $J$ satisfies the regularity conditions (a), (b-ii), and (c) of Definition 1.4. If $\mathcal{T}=$ $(M, I, \aleph ; j, \underline{A})$ is a bubble type such that $A=\sum_{i \in I} A_{i}$ and $A_{i} \neq 0$ for some minimal element $i$ of $I$, there exist $\delta, C \in C\left(\mathcal{U}_{\mathcal{T}}(X ; J) ; \mathbb{R}^{+}\right)$and an open neighborhood $U_{\mathcal{T}}$ of $\mathcal{U}_{\mathcal{T}}(X ; J)$ in $\mathfrak{X}_{1, M}(X, A)$ with the following properties: 
(1) For all $v=(b, v) \in \widetilde{\mathcal{F}}_{\delta}^{\varnothing}$,

$$
\begin{aligned}
\left\|\pi_{v ;-} \xi\right\|_{v, p, 1} & \leq C(b)\|\xi\|_{v, p, 1} & & \forall \xi \in \Gamma(v), \\
\left\|D_{J, v} \xi\right\|_{v, p} & \leq C(b)|v|^{1 / p}\|\xi\|_{v, p, 1} & & \forall \xi \in \Gamma-(v), \\
C(b)^{-1}\|\xi\|_{v, p, 1} & \leq\left\|D_{J, v} \xi\right\|_{v, p} \leq C(b)\|\xi\|_{v, p, 1} & & \forall \xi \in \Gamma_{+}(v) ;
\end{aligned}
$$

(2) For every $[\tilde{b}] \in \mathfrak{X}_{1, M}^{0}(X, A) \cap U_{\mathcal{T}}$, there exist $v=(b, v) \in \widetilde{\mathcal{F}} \mathcal{T}_{\delta}$ and $\zeta \in \Gamma_{+}(v)$ such that $\|\zeta\|_{v, p, 1}<\delta(b)$ and $\left[\exp _{b(v)} \zeta\right]=[\widetilde{b}]$.

This lemma is obtained by an argument analogous to that for Lemma 3.1. In particular, the bijectivity arguments in Section 4 of [16], with minor modifications, apply in the present situation.

Corollary 4.2 If $(X, \omega, J), A$, and $\mathcal{T}$ are as in Lemma 4.1 and $M=\varnothing$, for every precompact open subset $K$ of $\mathcal{U}_{\mathcal{T}}(X ; J)$, there exist $\delta_{K}, C_{K} \in \mathbb{R}^{+}$and an open neighborhood $U_{K} \subset U_{\mathcal{T}}$ of $K$ in $\mathfrak{X}_{1, \varnothing}(X, A)$ with the following properties:

(1) Requirements (1) and (2) of Lemma 4.1 are satisfied.

(2) If $\widetilde{J}$ is an almost complex structure on $X$ such that $\|\widetilde{J}-J\|_{C^{1}}<\delta_{K}$ and $[\tilde{b}] \in U_{K} \cap \mathfrak{X}_{1, \varnothing}^{0}(X, A)$, there exist a smooth Riemann surface $\Sigma$ and a smooth map $\tilde{u}: \Sigma \longrightarrow X$ such that $[\tilde{b}]=[\Sigma, \tilde{u}]$ and a linearization $D_{\widetilde{J}, \widetilde{u}}$ of $\bar{\partial} \widetilde{J}$ at $\tilde{u}$ is surjective.

The proof is identical to that for Corollary 3.2.

\subsection{A two-step gluing construction}

We prove the analogue of Corollary 4.2 for bubble types $\mathcal{T}=(\varnothing, I, \aleph ;, \underline{A})$ such that $A_{i}=0$ for all minimal elements $i$ of $I$, ie

$$
\mathcal{U}_{\mathcal{T}}(X ; J) \subset \overline{\mathfrak{M}}_{1, \varnothing}(X, A ; J)-\mathfrak{M}_{1, \varnothing}^{\{0\}}(X, A ; J),
$$

in the next subsection. In this subsection, we modify the gluing construction of [16] in two ways. First, we subdivide this construction into two steps. At the first stage, we use Lemma 3.3 to smooth out all nodes of the domain of a stable map that lie away from the principle component. At the second stage, we smooth out the remaining nodes, but at this step it may not be possible to perturb each approximately holomorphic map into a $J$-holomorphic map. The second modification is that the second-stage approximately holomorphic maps are closer to being holomorphic than they would be if constructed as in Sections 3.1 and 4.1 and in Section 3.3 of [16]. This modification is motivated 
by the pregluing construction of Section 3 in [6]. The two adjustments allow us to obtain estimates on the behavior of the operator $D_{\widetilde{J}, \tilde{u}}$ that are similar to the estimates of Corollary 3.6 for the operator $\mathfrak{D}_{\widetilde{J}, \tilde{u}}^{\infty, e_{\infty}}$.

If $\mathcal{T}=(M, I, \boldsymbol{\aleph} ; j, \underline{A})$ is a bubble type such that $A_{i}=0$ for all $i \in I_{0}$, let $I_{h} \subset I$, for $h \in I_{1}$, be as in Section 2.2. We put

$$
A_{h}(\mathcal{T})=\sum_{i \in I_{h}} A_{i}
$$

Let $\tilde{\pi}_{h}: \tilde{\mathcal{U}}_{\mathcal{T}}^{(0)}(X ; J) \longrightarrow \tilde{\mathcal{U}}_{\mathcal{T}_{h}}^{(0)}(X ; J)$ be the projection corresponding to the decomposition (2-11).

If $v=(b, v) \in \widetilde{\mathcal{F} \mathcal{T}}$, let

if

$$
\begin{gathered}
v_{0}=\left(b, v_{\aleph}, v_{0}\right), \quad v_{1}=\left(b, v_{1}\right), \quad v_{\{h\}}=\left(\tilde{\pi}_{h}(b), v_{\{h\}}\right) \text { for } h \in I_{1}, \\
v=\left(b, v_{\aleph}, v_{0}, v_{1}\right), \quad b \in \tilde{\mathcal{U}}_{\mathcal{T}}^{(0)}(X ; J), \quad\left(b, v_{\aleph}\right) \in \widetilde{\mathcal{F}_{\boldsymbol{\aleph}} \mathcal{T}},
\end{gathered}
$$

$$
\left(b, v_{0}\right) \in \widetilde{\mathcal{F}_{0} \mathcal{T}}, v_{1}=\left(v_{\{h\}}\right)_{h \in I_{1}} \in \bigoplus_{h \in I_{1}} \mathbb{C}^{\widehat{I}_{h}} .
$$

The component $v_{1}$ of $v$ consists of the smoothings of the nodes of $\Sigma_{b}$ that lie away from the principal component. In the case of Figure 5, these are the attaching nodes of the bubbles $h_{2}, h_{4}$, and $h_{5}$. The bubble map $b\left(v_{1}\right)$ for $v_{1} \in \widetilde{\mathcal{F}_{1} \mathcal{T}^{\varnothing}}$ is of bubble type

$$
\tilde{\mathcal{T}}=\left(M, I_{0} \cup I_{1}, \aleph ; j, \tilde{A}\right), \quad \text { where } \quad \tilde{A}_{i}= \begin{cases}0, & \text { if } i \in I_{0} ; \\ A_{i}(\mathcal{T}), & \text { if } i \in I_{1} .\end{cases}
$$

Similarly to (2-11) and (2-12), we put

$$
\begin{aligned}
& \mathcal{H}_{\widetilde{\mathcal{T}}}(X ; J)=\mathcal{U}_{T_{0}}(\mathrm{pt}) \times\left\{\left(b_{h}\right)_{h \in I_{1}} \in \prod_{h \in I_{1}} \mathcal{H}_{\widetilde{\mathcal{T}}_{h}}(X ; J):\right. \\
&\left.\operatorname{ev}_{0}\left(b_{h_{1}}\right)=\mathrm{ev}_{0}\left(b_{h_{2}}\right) \forall h_{1}, h_{2} \in I_{1}\right\}
\end{aligned},
$$

where $\mathcal{H}_{\widetilde{\mathcal{T}}_{h}}(X ; J)$ is the space of all $J$-holomorphic maps from $\mathbb{P}^{1}$ of type $\widetilde{\mathcal{T}}_{h}$. For each $h \in I_{1}, \delta \in \mathbb{R}^{+}$, and $v \in \widetilde{\mathcal{F} \mathcal{T}}$ as above, let

$$
\begin{aligned}
A_{v_{1}, h}^{-}(\delta) & =\left\{(h, z) \in\{h\} \times S^{2}:|z| \geq \delta^{-1 / 2} / 2\right\} \subset \Sigma_{v_{1}, h}, \\
\partial^{-} A_{v_{1}, h}^{-}(\delta) & =\left\{(h, z) \in\{h\} \times S^{2}:|z|=\delta^{-1 / 2} / 2\right\} \subset \Sigma_{v_{1}, h} .
\end{aligned}
$$

Finally, if $h \in I_{1}$ and $i \in \chi(\mathcal{T}) \cap I_{h}$, we put

$$
\tilde{\rho}_{i}(v) \equiv\left(b, \tilde{v}_{i}\right), \quad \text { where } \quad \tilde{v}_{i}=\prod_{h<i^{\prime} \leq i} v_{i^{\prime}} \in \mathbb{C} .
$$


In the case of Figure 5,

$$
\tilde{\rho}_{h_{1}}(v)=(b, 1), \quad \tilde{\rho}_{h_{4}}(v)=\left(b, v_{h_{4}}\right) \quad \text { and } \quad \tilde{\rho}_{h_{5}}(v)=\left(b, v_{h_{5}}\right) .
$$

Lemma 4.3 Suppose $(X, \omega, J)$ is a compact almost Kahler manifold, $A \in H_{2}(X ; \mathbb{Z})$, and $J$ is a genus-zero $A$-regular almost complex structure. If $\mathcal{T}=(M, I, \boldsymbol{\aleph} ; j, \underline{A})$ is a bubble type such that $\sum_{i \in I} A_{i}=A$ and $A_{i}=0$ for all minimal elements $i$ of $I$, for every precompact open subset $K$ of $\mathcal{U}_{\mathcal{T}}(X ; J)$, there exist $\delta_{K}, C_{K} \in \mathbb{R}^{+}$and an open neighborhood $U_{K}$ of $K$ in $\mathfrak{X}_{1, M}(X, A)$ with the following property. If $\widetilde{J}$ is an almost complex structure on $X$ such that $\|\widetilde{J}-J\|_{C^{1}} \leq \delta_{K}$, there exists a smooth map

$$
\tilde{\phi}_{\tilde{J} ; 1}:\left.\widetilde{\mathcal{F}}_{1}^{\varnothing} \delta_{K}\right|_{\tilde{K}^{(0)}} \longrightarrow \mathcal{H}_{\widetilde{\mathcal{T}}}(X ; \widetilde{J})
$$

such that

(1) the image of $\operatorname{Im} \widetilde{\phi}_{\widetilde{J} ; 1}$ under the quotient map $\mathcal{H}_{\widetilde{\mathcal{T}}}(X ; \widetilde{J}) \longrightarrow \mathcal{U}_{\widetilde{\mathcal{T}}}(X ; \widetilde{J})$ is $\mathcal{U}_{\widetilde{\mathcal{T}}}(X ; \widetilde{J}) \cap U_{K}$

(2) $\operatorname{ev}_{P}\left(\tilde{\phi}_{\widetilde{J} ; 1}\left(v_{1}\right)\right)=\operatorname{ev}_{P}(b)$ for all $v_{1}=\left.\left(b, v_{1}\right) \in{\widetilde{\mathcal{F}_{1} \mathcal{T}^{\prime}}}_{\delta_{K}}^{\varnothing}\right|_{\tilde{K}^{(0)}}$;

(3) if $\left.v_{1} \in \widetilde{\mathcal{F}}_{\mathcal{T}^{\prime}}^{\varnothing} \delta_{K}\right|_{\tilde{K}^{(0)}}, h \in I_{1}, \tilde{\pi}_{h}\left(\tilde{\phi}_{\widetilde{J} ; 1}\left(v_{1}\right)\right)=\left(\mathbb{P}^{1}, \tilde{u}_{v_{1}, h}\right)$, and $\delta \leq 4 \delta_{K}$,

$$
\left\|\left.d \tilde{u}_{v_{1}, h}\right|_{A_{v_{1}, h}^{-}(\delta)}\right\|_{v_{1}, p} \leq C_{K} \delta^{1 / p} \sum_{i \in \chi(\mathcal{T}) \cap I_{h}}\left|\tilde{\rho}_{i}(v)\right| .
$$

The smooth map $\widetilde{\phi}_{\widetilde{J} ; 1}$ is defined by

$$
\tilde{\pi}_{h}\left(\tilde{\phi}_{\widetilde{J} ; 1}\left(v_{1}\right)\right)=\left(\mathbb{P}^{1}, \exp _{u_{v_{\{h\}}}} \zeta_{\widetilde{J}, v_{\{h\}}}\right) \quad \forall h \in I_{1},
$$

where $v_{\{h\}}$ is as above and $\zeta \tilde{J}_{,} v_{\{h\}}$ is as in (2a) of Lemma 3.3. By (2c) of Lemma 3.3, the value of the map $\tilde{\pi}_{h}\left(\tilde{\phi}_{\tilde{J}_{; 1}}\left(v_{1}\right)\right)$ at the attaching node of the bubble $h$ is the same for all $h \in I_{1}$, as needed, and (2) of Lemma 4.1 is satisfied. The bound in (3) is simply a restatement of $(2 b)$ of Corollary 3.8 , since

$$
\left|\tilde{\rho}_{i}(v)\right|=\left|\rho_{i}\left(v_{\{h\}}\right)\right| \quad \forall h \in \chi(\mathcal{T}) \cap I_{1} .
$$

With notation as above, for each $v=(b, v)$, let

$$
\zeta_{\widetilde{J}, v_{1}} \in \Gamma\left(\Sigma_{v_{1}} ; u_{v_{1}}^{*} T X\right)
$$

be given by

$$
\zeta_{\tilde{J}_{, v_{1}}}(z)= \begin{cases}\zeta_{\tilde{J}, v_{\{h\}}}(z), & \text { if } z \in \Sigma_{v_{1}, h}, h \in I_{1} \\ 0, & \text { otherwise. }\end{cases}
$$


We write

$$
\tilde{\phi}_{\widetilde{J} ; 1}\left(v_{1}\right)=\tilde{b}_{\widetilde{J}}\left(v_{1}\right)=\left(\Sigma_{v_{1}}, \tilde{u}_{v_{1}}\right) \in \mathcal{H}_{\widetilde{\mathcal{T}}}(X ; \widetilde{J}) .
$$

The domain $\Sigma_{v_{1}}$ of the stable map $\tilde{b}_{\tilde{J}}\left(v_{1}\right)$ consists of the principal component $\Sigma_{v_{1}, \boldsymbol{\aleph}}$, which is either a smooth torus or a circle of spheres, and $\left|I_{1}\right|$ rational bubbles $\Sigma_{v, h}$, with $h \in I_{1}$, attached directly to $\Sigma_{v_{1}, \aleph}$. The $\widetilde{J}$-holomorphic map $\tilde{u}_{v_{1}}$ is constant on $\Sigma_{v_{1}, \aleph}$. Let

$$
\widetilde{\Gamma}\left(v_{1} ; \widetilde{J}\right)=L_{1}^{p}\left(\Sigma_{v_{1}} ; \tilde{u}_{v_{1}}^{*} T X\right) .
$$

We denote by $\Gamma_{\bar{\tau}}\left(v_{1} ; \widetilde{J}\right) \subset \widetilde{\Gamma}\left(v_{1} ; \widetilde{J}\right)$ the kernel of the linearization $D_{\widetilde{J}, \tilde{b}_{\widetilde{J}}\left(v_{1}\right)}$ of the



For each $h \in I_{1}, \xi \in \tilde{\Gamma}_{-}\left(\tilde{\pi}_{h}(b)\right)$, and $\tilde{\xi} \in \widetilde{\Gamma}\left(v_{\{h\}} ; \widetilde{J}\right)$ with $\tilde{\xi}(h, \infty)=0$, we define $\widetilde{R}_{b} \xi \in \Gamma_{-}(b)$ and $\widetilde{R}_{v_{1}} \tilde{\xi} \in \widetilde{\Gamma}\left(v_{1} ; \widetilde{J}\right)$ by

$$
\tilde{R}_{b} \xi(z)=\left\{\begin{array}{ll}
\xi(z), & \text { if } z \in \Sigma_{b_{h}} ; \\
0, & \text { otherwise; }
\end{array} \quad \widetilde{R}_{v_{1}} \tilde{\xi}(z)= \begin{cases}\tilde{\xi}(z), & \text { if } z \in \Sigma_{v_{1}, h} \\
0, & \text { otherwise }\end{cases}\right.
$$

see the paragraphs preceding Lemma 3.3 and Corollary 3.8 for notation. We put

$$
\begin{aligned}
\widetilde{\Gamma}_{-}(b) & =\left\{\widetilde{R}_{b} \xi: \xi \in \widetilde{\Gamma}_{-}\left(\tilde{\pi}_{h}(b)\right), h \in I_{1}\right\} \subset \Gamma_{-}(b), \\
\widetilde{\Gamma}_{-}\left(v_{1} ; \widetilde{J}\right) & =\left\{\widetilde{R}_{v_{1}} \xi: \xi \in \widetilde{\Gamma}_{-}\left(v_{\{h\}} ; \widetilde{J}\right), h \in I_{1}\right\} \subset \Gamma_{-}\left(v_{1} ; \widetilde{J}\right) .
\end{aligned}
$$

Let $\widetilde{R}_{v_{1}, \tilde{J}}: \widetilde{\Gamma}_{-}(b) \longrightarrow \widetilde{\Gamma}_{-}\left(v_{1} ; \widetilde{J}\right)$ be the homomorphism such that

$$
\widetilde{R}_{v_{1}, \widetilde{J}} \widetilde{R}_{b} \xi=\widetilde{R}_{v_{1}} \widetilde{R}_{v_{\{h\}}, \widetilde{J} \xi} \xi \forall \xi \in \widetilde{\Gamma}_{-}\left(\tilde{\pi}_{h}(b)\right), h \in I_{1},
$$

where $\widetilde{R}_{v_{\{h\}}, \tilde{J}}: \widetilde{\Gamma}_{-}\left(\tilde{\pi}_{h}(b)\right) \longrightarrow \Gamma_{-}\left(v_{\{h\}} ; \widetilde{J}\right)$ is the homomorphism defined just before Corollary 3.8.

If $v=\left.(b, v) \in \widetilde{\mathcal{F}}_{\delta_{K}}^{\varnothing}\right|_{\tilde{K}^{(0)}}$, let

$$
v_{\widetilde{J}}=\left(\widetilde{b}_{\widetilde{J}}\left(v_{1}\right), v_{\aleph}, v_{0}\right) .
$$

We denote by $\left(\Sigma_{v}, j_{v}\right)$ the smooth Riemann surface constructed as in Remark 2 of Section 4.1 and by

$$
q_{v_{0} ; 2}=q_{v_{\widetilde{J} ; \aleph}} \circ q_{v_{\widetilde{J}}^{\aleph}}: \Sigma_{v}=\Sigma_{\left(\tilde{b}_{\widetilde{J}}\left(v_{1}\right), v_{\aleph}, v_{0}\right)} \longrightarrow \Sigma_{v_{1}}=\Sigma_{\widetilde{b}_{\widetilde{J}}\left(v_{1}\right)},
$$

the corresponding basic gluing map. We next construct another map

$$
\tilde{q}_{v_{0} ; 2}=q_{v_{\widetilde{J} ; \aleph}} \circ \tilde{q}_{v_{\widetilde{J}}^{\aleph}}: \Sigma_{v} \longrightarrow \Sigma_{v_{1}}
$$

by defining the map

$$
\widetilde{q}_{v_{\widetilde{J}}^{\aleph}}: \Sigma_{v} \longrightarrow \Sigma_{\left(\widetilde{b}_{\widetilde{J}}\left(v_{1}\right), v_{\aleph}\right)}
$$


By construction, $\Sigma_{\left(\widetilde{b}_{\widetilde{J}}\left(v_{1}\right), v_{\aleph}\right)}$ is a smooth torus $\Sigma_{v_{\aleph}, \aleph}$ with $\left|I_{1}\right|$ bubbles attached at the points $\left\{x_{h}\left(v_{\aleph}\right)\right\}_{h \in I_{1}}$ of $\Sigma_{v_{\aleph}, \aleph}$. For each $h \in I_{1}$, we identify a small neighborhood $U_{h}\left(v_{\aleph}\right)$ of $x_{h}\left(v_{\aleph}\right)$ in $\Sigma_{v_{\aleph}, \boldsymbol{N}}$ with a neighborhood of $x_{h}\left(v_{\aleph}\right)$ in $T_{x_{h}\left(v_{\aleph}\right)} \Sigma_{v_{\aleph}, \boldsymbol{N}}$, biholomorphically and isometrically, with respect to the metric $g_{v_{\aleph}}$ on $\Sigma_{v_{\aleph}, \aleph}$ of Remark 2 in Section 4.1. We assume that all of these neighborhoods are disjoint from each other and from the $|\boldsymbol{N}|$ thin necks of $\Sigma_{v_{\boldsymbol{N}}, \boldsymbol{\aleph}}$. If $z \in U_{h}\left(v_{\boldsymbol{\aleph}}\right)$, we denote by $z-x_{h}\left(v_{\boldsymbol{N}}\right)$ the corresponding element of $T_{x_{h}\left(v_{\aleph}\right)} \Sigma_{v_{\aleph}, \aleph}$ and by $\left|z-x_{h}\left(v_{\aleph}\right)\right|$ its norm with respect to the metric $g_{v_{\mathbb{N}}}$. Let $\beta: \mathbb{R}^{+} \longrightarrow[0,1]$ be a smooth cutoff function such that

$$
\beta(r)=\left\{\begin{array}{ll}
0, & \text { if } r \leq 1 ; \\
1, & \text { if } r \geq 2 ;
\end{array} \quad \text { and } \quad \beta^{\prime}(r) \neq 0 \text { if } r \in(1,2) .\right.
$$

For each $\epsilon \in \mathbb{R}^{+}$, we define $\beta_{\epsilon} \in C^{\infty}(\mathbb{R} ; \mathbb{R})$ by $\beta_{\epsilon}(r)=\beta(r / \sqrt{\epsilon})$. If $\left|z-x_{h}\left(v_{\boldsymbol{\aleph}}\right)\right| \leq$ $2 \sqrt{\delta(b)}$, we put

$$
\begin{aligned}
& q_{v_{\widetilde{J}}^{\mathbf{N}} ; h}^{-}(z)=\left(1-\beta_{\delta_{K}}\left(2\left|z-x_{h}\left(v_{\boldsymbol{\aleph}}\right)\right|\right)\right) \overline{\left(\frac{v_{h}}{z-x_{h}\left(v_{\boldsymbol{\aleph}}\right)}\right)} \in \mathbb{C}, \\
& q_{v_{\widetilde{J}}^{+} ; h}^{+}(z)=x_{h}\left(v_{\boldsymbol{\aleph}}\right)+\beta_{\delta_{K}}\left(\left|z-x_{h}\left(v_{\boldsymbol{\aleph}}\right)\right|\right)\left(z-x_{h}\left(v_{\boldsymbol{\aleph}}\right)\right) \in \Sigma_{\left(\tilde{b}_{\widetilde{J}}\left(v_{1}\right), v_{\aleph}\right), \boldsymbol{\aleph}},
\end{aligned}
$$

where $v_{0}=\left(v_{h}\right)_{h \in I_{1}}$. By construction, the smooth Riemann surface $\Sigma_{v}$ is the main component $\Sigma_{\left(\widetilde{b}_{\widetilde{J}}\left(v_{1}\right), v_{\aleph}\right), \aleph}$ of $\Sigma_{\left(\widetilde{b}_{\widetilde{J}}\left(v_{1}\right), v_{\aleph}\right)}$. We define the map

$$
\begin{aligned}
& \widetilde{q}_{v \widetilde{J}}: \Sigma_{v} \longrightarrow \Sigma_{\left(\widetilde{b}_{\widetilde{J}}\left(v_{1}\right), v_{\aleph}\right)}, \\
& \widetilde{q}_{v_{\widetilde{J}}^{\aleph}}(z)= \begin{cases}\left(h, q_{S}\left(q_{v_{\widetilde{J}}^{\aleph} ; h}^{-}(z)\right)\right) \in \Sigma_{\left(\tilde{b}_{\widetilde{J}}\left(v_{1}\right), v_{\aleph}\right), h}, & \text { if }\left|z-x_{h}\left(v_{\aleph}\right)\right| / \sqrt{\delta_{K}} \leq 1, h \in I_{1} ; \\
q_{v_{\widetilde{J}}^{\aleph} ; h}^{+}(z) \in \Sigma_{\left(\tilde{b}_{\widetilde{J}}\left(v_{1}\right), v_{\aleph}\right), \aleph}, & \text { if } 1 \leq\left|z-x_{h}\left(v_{\aleph}\right)\right| / \sqrt{\delta_{K}} \leq 2, h \in I_{1} ; \\
z \in \Sigma_{\left(\tilde{b}_{\widetilde{J}}\left(v_{1}\right), v_{\aleph}\right), \aleph}, & \text { otherwise, }\end{cases}
\end{aligned}
$$

where $q_{S}: \mathbb{C} \longrightarrow S^{2}$ is the standard (antiholomorphic) stereographic projection taking the origin in $\mathbb{C}$ to the south pole in $S^{2}$. Like the map $q_{v_{0} ; 2}, \widetilde{q}_{v_{0} ; 2}$ smooths out the nodes of the principal component and stretches small neighborhoods of the points $x_{h}\left(v_{\aleph}\right)$ around the $\left|I_{1}\right|$ bubbles. Furthermore,

$$
\left\|d \widetilde{q}_{v_{0} ; 2}\right\|_{C^{0}} \leq C(b),
$$

for some $C \in C^{\infty}\left(\mathcal{U}_{\mathcal{T}}(X ; J) ; \mathbb{R}^{+}\right)$, if the norm is taken with respect to the metrics $g_{v}$ on $\Sigma_{v}$ and $g_{v_{1}}$ on $\Sigma_{v_{1}}$, constructed via the basic gluing maps $q_{v}$ and $q_{v_{1}}$, respectively; see Section 3.3 in [16]. The map $\widetilde{q}_{v_{0} ; 2}$ is a homeomorphism outside of $|\boldsymbol{\aleph}|+\left|I_{1}\right|$ circles of $\Sigma_{v}$ and is biholomorphic outside of the $|\boldsymbol{\aleph}|$ thin necks corresponding to the nodes of the principal component of $\Sigma_{\aleph}$ and the $\left|I_{1}\right|$ annuli $\widetilde{\mathcal{A}}_{b, h}^{-} \cup \widetilde{\mathcal{A}}_{b, h}^{+}$with $h \in I_{1}$, 
where

$$
\begin{aligned}
& \tilde{\mathcal{A}}_{b, h}^{-}=\left\{z \in \Sigma_{v}: 1 / 2 \leq\left|z-x_{h}\left(v_{\aleph}\right)\right| / \sqrt{\delta_{K}} \leq 1\right\}, \\
& \tilde{\mathcal{A}}_{b, h}^{+}=\left\{z \in \Sigma_{v}: 1 \leq\left|z-x_{h}\left(v_{\aleph}\right)\right| / \sqrt{\delta_{K}} \leq 2\right\} .
\end{aligned}
$$

The key advantage of the map $\widetilde{q}_{v_{0} ; 2}$ over $q_{v_{0} ; 2}$ is that

$$
\left\|d \widetilde{q}_{v_{0} ; 2}\right\|_{C^{0}\left(\tilde{\mathcal{A}}_{b, h}^{-}\right)} \leq C(b)\left|v_{h}\right| \quad \forall h \in I_{1} ;
$$

this bound is immediate from the definition of the norms.

If $\tilde{\phi}_{\tilde{J} ; 1}\left(v_{1}\right)=\left(\Sigma_{v_{1}}, \tilde{u}_{v_{1}}\right)$ as above, we take

$$
b_{\widetilde{J}}(v)=\left(\Sigma_{v}, j_{v}, u_{v}\right), \quad \text { where } \quad u_{v}=\tilde{u}_{v_{1}} \circ \widetilde{q}_{v_{0} ; 2},
$$

to be the approximately $\widetilde{J}$-holomorphic map corresponding to the gluing parameter $v$ at the present, second, stage of the gluing construction. By (4-6) and (4-7),

$$
\begin{aligned}
\left\|d u_{v}\right\|_{v, p} & \leq C(b)\left\|d \tilde{u}_{v_{1}}\right\|_{v_{1}, p}, \\
\left\|\bar{\partial} \widetilde{J} u_{v}\right\|_{v, p} & \leq C(b) \sum_{h \in I_{1}}\left\|\left.d \widetilde{u}_{v_{1}}\right|_{\mathcal{A}_{v, h}^{-}}\right\|_{v_{1}, p}\left|v_{h}\right|^{\frac{p-2}{p}},
\end{aligned}
$$

where $\mathcal{A}_{v, h}^{-}=\tilde{q}_{v_{0} ; 2}\left(\tilde{\mathcal{A}}_{b, h}^{-}\right) \subset \Sigma_{v_{1}}$ and $\|\cdot\|_{v, p}$ and $\|\cdot\|_{v_{1}, p}$ are the norms corresponding to the basic gluing maps $q_{v}$ and $q_{v_{1}}$; see [16, Section 3.3]. The second bound follows from the fact that the map $\tilde{u}_{v_{1}}$ is $\widetilde{J}$-holomorphic on $\Sigma_{v_{1}}$ and is constant on the principal component of $\Sigma_{v_{1}}$; thus, $\bar{\partial} \widetilde{J} u_{v}$ is supported on the annuli $\widetilde{\mathcal{A}}_{b, h}^{-}$, with $h \in I_{1}$. If $v=(b, v)$, we denote by

$$
\Gamma(v)=L_{1}^{p}\left(\Sigma_{v} ; u_{v}^{*} T X\right) \quad \text { and } \quad \Gamma^{0,1}(v ; \widetilde{J})=L^{p}\left(\Sigma_{v} ; \Lambda_{\widetilde{J}, j_{v}}^{0,1} T^{*} \Sigma_{v} \otimes u_{v}^{*} T X\right)
$$

the Banach completions of the corresponding spaces of smooth sections with respect to the norms $\|\cdot\|_{v, p, 1}$ and $\|\cdot\|_{v, p}$, induced from the basic gluing map $q_{v}: \Sigma_{v} \longrightarrow \Sigma_{b}$, as before, and the $\widetilde{J}$-compatible metric

$$
g_{\widetilde{J}}(\cdot, \cdot) \equiv \frac{1}{2}\left(g_{X}(J \cdot, \tilde{J} \cdot)+g_{X}(\tilde{J} \cdot, J \cdot)\right)
$$

on $X$. We put

$$
\begin{aligned}
& \Gamma_{-}(v ; \widetilde{J})=\left\{R_{v_{0}} \xi: \xi \in \Gamma_{-}\left(v_{1} ; \widetilde{J}\right)\right\} \subset \Gamma(v), \\
& \widetilde{\Gamma}_{-}(v ; \widetilde{J})=\left\{R_{v_{0}} \xi: \xi \in \widetilde{\Gamma}_{-}\left(v_{1} ; \widetilde{J}\right)\right\} \subset \Gamma_{-}(v ; \widetilde{J}),
\end{aligned}
$$

where $R_{v_{0}} \xi=\xi \circ \tilde{q}_{v_{0} ; 2}$. Let

$$
R_{v, \tilde{J}}=R_{v_{0}} \circ \widetilde{R}_{v_{1}, \tilde{J}}: \widetilde{\Gamma}_{-}(b) \longrightarrow \widetilde{\Gamma}_{-}(v ; \widetilde{J}) .
$$


With $\zeta_{\widetilde{J}, v_{1}}$ as in (4-3), we set

$$
\Gamma_{-}(v)=\left\{R_{v_{0}} \Pi_{\zeta_{\widetilde{J}, v_{1}}}\left(\xi \circ q_{v_{1}}\right): \xi \in \Gamma_{-}(b)\right\} \subset \Gamma(v)
$$

We denote by $\Gamma_{+}(v)$ the $L^{2}$-orthogonal complement of $\Gamma_{-}(v)$ in $\Gamma(v)$, as in Sections 3.1 and 4.1 .

It remains to describe the obstruction bundle, ie a complement to the image of $\Gamma_{+}(v)$ under $D_{J, v}$, or $D_{\widetilde{J}, v}$ if $\widetilde{J}$ is sufficiently close to $J$. First, we describe the Fredholm situation along $\tilde{\mathcal{U}}_{\mathcal{T}}^{(0)}(X ; J)$. The linearization $D_{J, b}$ of the $\bar{\partial}_{J}$-operator along $\tilde{\mathcal{U}}_{\mathcal{T}}^{(0)}(X ; J)$ is not surjective. From the decomposition (2-11) and the regularity conditions of Definition 1.3, we see that the cokernel of $D_{J, b}$, for $b \in \tilde{\mathcal{U}}_{\mathcal{T}}^{(0)}(X ; J)$, can be identified with the vector space

$$
\Gamma_{-}^{0,1}(b ; J) \approx \mathbb{E}_{\widetilde{\pi}_{P}(b)}^{*} \otimes_{J} T_{\operatorname{ev}_{P}(b)} X
$$

of $\left(T_{\mathrm{ev}_{P}(b)} X, J\right)$-harmonic antilinear differentials on the main component $\Sigma_{b, \aleph}$. In other words, if $\psi \in \mathcal{H}_{b, \aleph}$ is a nonzero harmonic $(0,1)$-form on $\Sigma_{b, \boldsymbol{\aleph}}$,

$$
\Gamma_{-}^{0,1}(b ; J)=\left\{Y \cdot J \psi: Y \in T_{\mathrm{ev}_{P}(b)} X\right\} .
$$

If $\boldsymbol{\aleph} \neq \varnothing$, ie $\Sigma_{b, \boldsymbol{\aleph}}$ is a circle of spheres, the elements of $\Gamma_{-}^{0,1}(b ; J)$ have simple poles at the nodes of $\Sigma_{b, \aleph}$ with the residues adding up to zero at each node. Let

$$
\Gamma_{-}^{0,1}(b ; \widetilde{J}) \approx \mathbb{E}_{\tilde{\pi}_{P}(b)}^{*} \otimes \tilde{J}_{\operatorname{ev}_{P}(b)} X
$$

be the vector space of $\left(T_{\mathrm{ev}_{P}(b)} X, \widetilde{J}\right)$-harmonic differentials on the main component $\Sigma_{b, \aleph}$ of $\Sigma_{b}$. If $v=\left.(b, v) \in \widehat{\mathcal{F}}_{\delta_{K}}^{\varnothing}\right|_{\widetilde{K}^{(0)}}$, with notation as above, let

$$
\Gamma_{-}^{0,1}\left(v_{\boldsymbol{\aleph}} ; \widetilde{J}\right) \approx \mathbb{E}_{\Sigma_{v_{\mathbb{S}}, \aleph}^{*}}^{*} \otimes \widetilde{J}_{\operatorname{ev}_{P}(b)} X
$$

be the space of $\left(T_{\mathrm{ev}_{P}(b)} X, \widetilde{J}\right)$-harmonic differentials on the main component $\Sigma_{v_{\aleph}, \aleph}$ of $\Sigma_{v_{\aleph}}$. If $\boldsymbol{\aleph}=\varnothing, \Gamma_{-}^{0,1}\left(v_{\aleph} ; \widetilde{J}\right) \equiv \Gamma_{-}^{0,1}(b ; \widetilde{J})$.

We now construct a homomorphism

$$
R_{v}^{\aleph}: \Gamma_{-}^{0,1}\left(v_{\aleph} ; \widetilde{J}\right) \longrightarrow \Gamma^{0,1}(v ; \widetilde{J}) .
$$

For each $h \in I_{1}$ and $z \in A_{v_{1}, h}^{-}\left(4 \delta_{K}\right)$, we define

$$
\tilde{\zeta}_{b ; v}(z) \in T_{\operatorname{ev}_{P}(b)} X \quad \text { by } \quad \exp _{\operatorname{ev}_{P}(b)}^{\widetilde{J}} \tilde{\zeta}_{b ; v}(z)=\tilde{u}_{v_{1}}(z),\left|\widetilde{\zeta}_{b ; v}(z)\right|<r_{\widetilde{J}} \text {, }
$$


where $\exp ^{\tilde{J}}$ is the exponential map for the connection $\nabla^{\widetilde{J}}$ and $r \widetilde{J}$ is its injectivity radius. If $\eta \in \Gamma_{-}^{0,1}\left(v_{\aleph} ; \widetilde{J}\right)$, we define $R_{v}^{\aleph} \eta \in \Gamma^{0,1}(v ; \widetilde{J})$ by

$$
\begin{aligned}
& \left\{R_{v}^{\aleph} \eta\right\}_{z} w \\
& = \begin{cases}0, & \text { if } \sqrt{\delta_{K}} \frac{\left|z-x_{h}\left(v_{\aleph}\right)\right|}{\left|v_{h}\right|} \leq \frac{1}{4}, h \in I_{1} ; \\
\beta_{\frac{\left|v_{h}\right|^{2}}{\delta_{K}}\left(4\left|z-x_{h}\left(v_{\aleph}\right)\right|\right) \prod_{\widetilde{\zeta}_{b ; v}}^{\widetilde{J}} \widetilde{q}_{v_{0} ; 2}(z)} \eta_{z}(w), & \text { if } \frac{1}{4} \leq \sqrt{\delta_{K}} \frac{\left|z-x_{h}\left(v_{\aleph}\right)\right|}{\left|v_{h}\right|} \leq \frac{1}{2}, h \in I_{1} ; \\
\eta_{z}(w), & \text { otherwise, }\end{cases}
\end{aligned}
$$

for all $z \in \Sigma_{v}$ and $w \in T_{z} \Sigma_{v}$, where $\Pi^{\widetilde{J}}$ denotes the parallel transport of the connection $\nabla^{\widetilde{J}}$. Let $\Gamma_{-}^{0,1}(v ; \widetilde{J})$ denote the image of $\Gamma_{-}^{0,1}\left(v_{\aleph} ; \widetilde{J}\right)$ under $R_{v}^{\aleph}$.

If $\eta \in \Gamma_{-}^{0,1}(b ; \widetilde{J})$ and $\widetilde{\eta} \in \Gamma_{-}^{0,1}\left(v_{\aleph} ; \widetilde{J}\right)$, we put

$$
\|\eta\|=\sum_{h \in I_{1}}|\eta|_{x_{h}(b)} \quad \text { and } \quad\|\tilde{\eta}\|=\sum_{h \in I_{1}}|\widetilde{\eta}|_{x_{h}\left(\widetilde{b}_{\widetilde{J}}\left(v_{1}\right), v_{\aleph}\right)}
$$

where $|\eta|_{x_{h}(b)}$ is the norm of $\left.\eta\right|_{x_{h}(b)}$ with respect to the metric $g_{\tilde{J}}$ on $X$ and the metric $g_{\widetilde{\pi}_{P}(b)}$ on $\Sigma_{b, \aleph}$. Similarly, $|\widetilde{\eta}|_{x_{h}\left(\widetilde{b}_{\widetilde{J}}\left(v_{1}\right), v_{\aleph}\right)}$ denotes the norm of $\left.\tilde{\eta}\right|_{x_{h}\left(\widetilde{b}_{\widetilde{J}}\left(v_{1}\right), v_{\aleph}\right)}$ with respect to $g_{\widetilde{J}}$ and the metric $g_{\left(\tilde{\pi}_{P}(b), v_{\aleph}\right)}$ on $\Sigma_{\left(\tilde{b}_{\widetilde{J}}\left(v_{1}\right), v_{\aleph}\right), \aleph}=\Sigma_{\left(\tilde{\pi}_{P}(b), v_{\aleph}\right)}$. If $\boldsymbol{\aleph} \neq \varnothing$, we can obtain an isomorphism

$$
R_{v_{\aleph}}: \Gamma_{-}^{0,1}(b ; \widetilde{J}) \longrightarrow \Gamma_{-}^{0,1}\left(v_{\aleph} ; \widetilde{J}\right)
$$

by requiring that

$$
\left\{R_{v_{\aleph}} \eta\right\}_{x_{h}\left(v_{\aleph}\right)}=\left.\left.d q_{v_{\aleph}}\right|_{x_{h}\left(v_{\aleph}\right)} ^{*} \eta\right|_{x_{h}(b)} \quad \forall \eta \in \Gamma_{-}^{0,1}(b ; \widetilde{J}), h \in I_{1} \cdot{ }^{4}
$$

If $\aleph=\varnothing$, we take $R_{v_{\aleph}}$ to be the identity map. In either case, we denote by

$$
R_{v}: \Gamma_{-}^{0,1}(b ; \widetilde{J}) \longrightarrow \Gamma_{-}^{0,1}(v ; \widetilde{J})
$$

the composition $R_{v}^{\aleph} \circ R_{v_{\aleph}}$. It is immediate from this construction that for every $q \in[1,2)$,

$$
\left\|R_{v} \eta\right\|_{v, q} \leq C_{q}\|\eta\| \quad \forall \eta \in \Gamma_{-}^{0,1}(b ; \widetilde{J}) .^{5}
$$

\footnotetext{
${ }^{4} \mathrm{~A}$ harmonic $(0,1)$-form on the circle of spheres $\Sigma_{b, \boldsymbol{N}}$ is determined by its value at any smooth point; the same is the case for a harmonic $(0,1)$-form on the smooth torus $\Sigma_{v_{\boldsymbol{N}}, \boldsymbol{\aleph}}$. Thus, (4-9) with a fixed $h \in I_{1}$ determines $R_{v_{\mathbb{N}}}$. On the other hand, one can easily choose $\widetilde{\sigma}_{\mathcal{T}_{0}}$ in (4-1) so that (4-9) can be satisfied for all $h \in I_{1}$ at the same time.

${ }^{5}$ The $L^{q}$-norm, with $q<2$, of a harmonic $(0,1)$-form on $\Sigma_{b, \aleph}$ is finite and is determined by the value of the form at any smooth point.
} 
Finally, we denote by $D_{J, \underline{v}}$ the linearization of $\bar{\partial}_{J}$ at $b_{\widetilde{J}}(v)$ defined via $\nabla^{J}$ and by $D_{\widetilde{J}, v}$ the linearization of $\frac{\bar{\partial}}{\tilde{J}}$ at $b_{\widetilde{J}}(v)$ defined via $\nabla^{\widetilde{J}}$. Let $D_{\widetilde{J}, v}^{*}$ denote the formal adjoint of $D_{\widetilde{J}, v}$, defined with respect to the metrics $g_{v}$ on $\Sigma_{v}$ and $g_{\widetilde{J}}$ on $X$; see Chapter 3 in [8]. For any $h \in I_{1}$ and $\delta \in \mathbb{R}^{+}$, we take

$$
\begin{aligned}
A_{v_{\aleph}, h}^{+}(\delta) & =\left\{z \in \Sigma_{v_{\aleph}, \aleph}:\left|z-x_{h}\left(v_{\aleph}\right)\right| \leq 2 \delta^{1 / 2}\right\} \subset \Sigma_{v_{\widetilde{J} ; \aleph}, \aleph}, \\
\tilde{A}_{v, h}^{+}(\delta) & =q_{v_{\widetilde{J}}^{\aleph}}^{-1}\left(A_{v_{\aleph}, h}^{+}(\delta)\right), \\
\tilde{A}_{v, h}^{-}(\delta) & =q_{v_{0} ; 2}^{-1}\left(A_{v_{1}, h}^{-}(\delta)\right)=q_{v}^{-1}\left(A_{b, h}^{-}(\delta)\right) \subset \Sigma_{v},
\end{aligned}
$$

and

$$
\partial^{-} \tilde{A}_{v, h}^{-}(\delta)=q_{v_{0} ; 2}^{-1}\left(\partial^{-} A_{v_{1}, h}^{-}(\delta)\right),
$$

where $A_{v_{1}, h}^{-}(\delta)$ and $\partial^{-} A_{v_{1}, h}^{-}(\delta)$ are as in the paragraph preceding Lemma 4.3. If $Y_{1}, Y_{2} \in T_{q} X$ for some $q \in X$, we put

$$
\left\langle Y_{1}, Y_{2}\right\rangle_{\widetilde{J}}=g_{\widetilde{J}}\left(Y_{1}, Y_{2}\right)+i g_{\widetilde{J}}\left(Y_{1}, \widetilde{J} Y_{2}\right) \in \mathbb{C} .
$$

Similarly, if $\eta_{1}, \eta_{2} \in \Gamma^{0,1}(v ; \widetilde{J})$, we put

$$
\left\langle\left\langle\eta_{1}, \eta_{2}\right\rangle\right\rangle=\left\langle\left\langle\eta_{1}, \eta_{2}\right\rangle\right\rangle_{v, 2}+\mathfrak{i}\left\langle\left\langle\eta_{1}, \tilde{J} \eta_{2}\right\rangle\right\rangle_{v, 2} \in \mathbb{C},
$$

where $\langle\langle\cdot, \cdot\rangle\rangle_{v, 2}$ is the (real-valued) $L^{2}$-inner product on $\Gamma^{0,1}(v ; \widetilde{J})$ with respect to the metric $g_{\widetilde{J}}$ on $X$. Note that by Holder's inequality and (4-10)

$$
\left|\left\|\eta^{\prime}, R_{v} \eta\right\|_{v}\right| \leq C\|\eta\|\left\|\eta^{\prime}\right\|_{v, p} \quad \forall \eta \in \Gamma_{-}^{0,1}(b ; \widetilde{J}), \eta^{\prime} \in \Gamma^{0,1}(v ; \widetilde{J}) .
$$

Lemma 4.4 If $(X, \omega, J), A$, and $\mathcal{T}$ are as in Lemma 4.3, for every precompact open subset $K$ of $\mathcal{U}_{\mathcal{T}}(X ; J)$, there exist $\delta_{K}, C_{K} \in \mathbb{R}^{+}$and an open neighborhood $U_{K}$ of $K$ in $\mathfrak{X}_{1, M}(X, A)$ with the following property. If $\widetilde{J}$ is an almost complex structure on $X$ such that $\|\widetilde{J}-J\|_{C^{1}} \leq \delta_{K}$,

(1) the second-stage pregluing map, $v \longrightarrow b_{\widetilde{J}^{(v)}}$, is defined on $\left.\widetilde{\mathcal{F}}_{\delta_{K}}^{\varnothing}\right|_{\widetilde{K}^{(0)}}$;

(2) for every $[\tilde{b}] \in \mathfrak{X}_{1, M}^{0}(X, A) \cap U_{K}$, there exist $\left.v \in \widetilde{\mathcal{F}} \mathcal{T}_{\delta_{K}}^{\varnothing}\right|_{K^{(0)}}$ and $\zeta \in \Gamma_{+}(v)$ such that $\|\zeta\|_{v, p, 1}<\delta_{K}$ and $\left[\exp _{b_{\widetilde{J}}(v)}^{\widetilde{J}} \zeta\right]=[\tilde{b}]$;

(3) for all $v=\left.(b, v) \in \widetilde{\mathcal{F}}_{\delta_{K}}^{\varnothing}\right|_{\tilde{K}^{(0)}}$,

$\left\|\bar{\partial} \widetilde{J} u_{v}\right\|_{v, p} \leq C_{K}|\rho(v)|, \quad\left\|D_{\widetilde{J}, v} \xi\right\|_{v, p} \leq C_{K}|v|^{(p-2) / p}\|\xi\|_{v, p, 1} \quad \forall \xi \in \Gamma_{-}(v ; \widetilde{J})$, and

$$
C_{K}^{-1}\|\xi\|_{v, p, 1} \leq\left\|D_{\widetilde{J}, v} \xi\right\|_{v, p} \leq C_{K}\|\xi\|_{v, p, 1} \quad \forall \xi \in \Gamma_{+}(v)
$$

(4) for all $v=\left.(b, v) \in \widetilde{\mathcal{F}}_{\delta_{K}}^{\varnothing}\right|_{\widetilde{K}^{(0)}}, h \in I_{1}$, and $\xi \in \widetilde{\Gamma}_{-}(v ; \widetilde{J})$,

$\left\|D_{\widetilde{J}, v} \xi\right\|_{v, p} \leq C_{K}|\rho(v)| \cdot\|\xi\|_{v, p, 1}, \quad\left\|\left.\xi\right|_{\tilde{A}_{v, h}^{+}\left(\delta_{K}\right)}\right\|_{v, p, 1} \leq C_{K}|v|^{1 / p}|\rho(v)| \cdot\|\xi\|_{v, p, 1}$ 
(5) for all $v=\left.(b, v) \in \widetilde{\mathcal{F}}_{\delta_{K}}^{\varnothing}\right|_{\widetilde{K}^{(0)}}, \xi \in \widetilde{\Gamma}_{-}(b)$, and $\eta \in \Gamma_{-}^{0,1}(b ; \widetilde{J})$,

$$
\begin{aligned}
& \left\|R_{v, \widetilde{J}} \xi\right\|_{v, p, 1} \leq\|\xi\|_{b, p, 1}, \quad\left\|\left.R_{v} \eta\right|_{\tilde{A}_{v, h}^{-}\left(4 \delta_{K}\right)}\right\|_{v, 2} \leq C_{K}|v|^{1 / 2}\|\eta\| \quad \forall h \in I_{1}, \\
& \mid\left\langle D_{\widetilde{J}, v} R_{v, \widetilde{J}} \xi, R_{v} \eta \|+2 \pi \mathfrak{i} \sum_{i \in \chi(\mathcal{T})}\left|\mathfrak{D}_{J, b ; i} \xi, \eta_{x_{h(i)}(b)}\left(\rho_{i}(v)\right)\right|_{b}\right| \\
& \leq C_{K}\left(\|\widetilde{J}-J\|_{C^{1}}+|v|^{1 / p}+|v|^{(p-2) / p}\right)|\rho(v)| \cdot\|\eta\|\|\xi\|_{b, p, 1}
\end{aligned}
$$

(6) for all $v=(b, v) \in{\widetilde{\mathcal{F}} \mathcal{T}_{\delta_{K}}^{\varnothing}}_{\widetilde{K}^{(0)}}$ and $\eta \in \Gamma_{-}^{0,1}(b ; \widetilde{J})$,

$$
\begin{aligned}
& \left|\left\langle\bar{\partial} \widetilde{J} u_{v}, R_{v} \eta\right\rangle+2 \pi \mathfrak{i} \sum_{i \in \chi(\mathcal{T})}\left\langle\mathcal{D}_{J, i} \rho_{i}(v), \eta_{x_{h(i)}(b)}\right\rangle_{b}\right| \\
& \quad \leq C_{K}\left(\|\widetilde{J}-J\|_{C^{1}}+|v|^{1 / p}+|v|^{(p-2) / p}\right)|\rho(v)| \cdot\|\eta\| ;
\end{aligned}
$$

(7) for all $v=\left.(b, v) \in \widetilde{\mathcal{F}}_{\delta_{K}}^{\varnothing}\right|_{\widetilde{K}^{(0)}}, \xi \in \Gamma(v)$, and $\eta \in \Gamma_{-}^{0,1}(b ; \widetilde{J})$,

$$
\left.\left|\left\langle D_{\widetilde{J}, v} \xi, R_{v} \eta\right\rangle\right\rangle_{v, 2}\left|\leq C_{K}\right| v\right|^{1 / 2}\|\eta\|\|\xi\|_{v, p, 1}
$$

Remark In (6) above, $\langle\cdot, \cdot\rangle_{b}$ denotes the combination of the inner-product defined before Lemma 4.4 with a contraction. More precisely,

$$
\left\langle\mathcal{D}_{\widetilde{J}_{, i}}(b, v), \eta_{x}\right\rangle_{b}=\overline{\psi_{x}(v)}\left\langle\mathcal{D}_{i} b, Y\right\rangle \quad \text { if } \quad \eta=\psi \otimes Y \in \mathbb{E}^{*} \otimes \mathrm{ev}_{P}^{*} T X ;
$$

see the paragraph preceding Lemma 3.5.

The first statement of this lemma is essentially a restatement of Lemma 4.3, in the light of the constructions following Lemma 4.3. In (2),

$$
\exp _{b_{\widetilde{J}}(v)}^{\widetilde{J}} \zeta=\left(\Sigma_{v}, j_{v}, \exp _{u_{v}}^{\widetilde{J}} \zeta\right), \quad \text { if } \quad b_{\widetilde{J}}(v)=\left(\Sigma_{v}, j_{v}, u_{v}\right)
$$

The arguments of Section 4 in [16] can be modified, in a straightforward way, to show that for every $[\tilde{b}] \in \mathfrak{X}_{1, M}^{0}(X, A)$ sufficiently close to $\mathcal{U}_{\mathcal{T}}(X ; J)$, there exists a pair $(v, \zeta)$ as in (2) of Lemma 4.4 and this pair is unique up to the action of the group $\operatorname{Aut}(\mathcal{T}) \propto\left(S^{1}\right)^{\hat{I}}$, ie the present two-stage gluing construction retains the essential bijectivity property of the one-stage gluing construction in [16]. The key point is that the metrics $g_{v}$ on $\Sigma_{v}$ and the weights used to modify the standard Sobolev norms, as in Section 3.3 of [16], are the same in the one-stage gluing construction and in the present case, while the difference between the data appearing in the two constructions is very small. 
The first bound in (3) of Lemma 4.4 is immediate from the second bound in (4-8) and $(2 \mathrm{~b})$ of Corollary 3.8 , since

$$
\tilde{q}_{v_{0} ; 2}\left(\tilde{\mathcal{A}}_{b, h}^{-}\right)=\mathcal{A}_{v, h}^{-} \subset A_{v_{1}, h}^{-}\left(\left|v_{h}\right|^{2} / \delta_{K}\right)
$$

The two bounds in (4) follow from (2e) of Corollary 3.8 in a similar way. The second estimate in (3) is obtained by the same argument as the second bound in (4-8). The final claim of (3) is a consequence of the analogous inequalities for $D_{J, v}$; see Section 3.5 in [16]. The first inequality in (5) is clear from the first inequality in (2d) of Corollary 3.8. For the second one, it is enough to observe that the $L^{2}$-norm of a one-form is invariant under conformal changes of the metric on a two-dimensional domain, while the larger radius of the annulus $\tilde{A}_{v, h}^{-}(\delta)$ is $\left|v_{h}\right|^{1 / 2}$, with respect to the metric $g_{v_{\aleph}}$ on $\Sigma_{v_{\aleph}, \kappa}$.

For the remaining three estimates, we observe that for any $h \in I_{1}$,

$$
\begin{array}{cr}
\left|D_{\widetilde{J}, v}^{*} R_{v} \eta\right|_{g_{v}, z} \leq C_{K}\left|d u_{v}\right|_{g_{v}, z}\|\eta\| & \forall z \in \widetilde{A}_{v, h}^{+}\left(\delta_{K}\right), \\
\left|D_{\widetilde{J}, v}^{*} R_{v} \eta\right|_{g_{v}, w_{h}} \leq C_{K}\left|d u_{v}\right|_{g_{v}, w_{h}} \frac{\left|v_{h}\right|}{\left|w_{h}\right|}\|\eta\| & \forall w_{h} \in \widetilde{A}_{v, h}^{-}\left(\delta_{K}\right), \\
\left|D_{\widetilde{J}, v}^{*} R_{v} \eta\right|_{g_{v}, w_{h}} \leq C_{K}\left(1+\left|d u_{v}\right|_{g_{v}, w_{h}}\right)\left|v_{h}\right|\|\eta\| & \forall w_{h} \in \widetilde{A}_{\bar{v}, h}^{-}\left(4 \delta_{K}\right) \\
& -\tilde{A}_{v, h}^{-}\left(\delta_{K}\right),
\end{array}
$$

where $z$ is a holomorphic coordinate on a neighborhood of $x_{h}\left(v_{\aleph}\right)$ in $\Sigma_{\aleph}$, which is unitary with respect to the metric $g_{v_{\aleph}}$ on $\Sigma_{v_{\aleph}, \aleph}, w_{h}=v_{h} / z$, and $|z|$ denotes the norm in the standard metric on $\mathbb{C}$. These estimates are obtained by a direct computation from an explicit expression for $D_{\widetilde{J}, v}^{*}$, such as the one in Chapter 3 of [8], and simple facts of Riemannian geometry, such as those in Section 2.1 of [14]. The difference between (4-13) and (4-14) is due to the fact that the cutoff function used in the construction of $R_{v} \eta$ is constant outside of the annuli $\tilde{A}_{v, h}^{-}\left(4 \delta_{K}\right)-\tilde{A}_{v, h}^{-}\left(\delta_{K}\right)$, with $h \in I_{1}$. An explicit computation of the contribution of this cutoff function on $\widetilde{A}_{v, h}^{-}\left(4 \delta_{K}\right)-\tilde{A}_{v, h}^{-}\left(\delta_{K}\right)$ is given in Section 2.2 of [13]. From the definition of the map $\tilde{q}_{v_{0} ; 2}$ and the metric $g_{v}$, it is easy to see that

$$
\begin{gathered}
\left|d \widetilde{q}_{v_{0} ; 2}\right|_{g_{v}, z} \leq 4 \frac{\left|v_{h}\right|}{|z|^{2}} \quad \forall z \in \tilde{A}_{v, h}^{+}\left(\delta_{K}\right), \\
\left|d \widetilde{q}_{v_{0} ; 2}\right|_{g_{v}, w_{h}} \leq 4 \quad \forall w_{h}=\frac{v_{h}}{z} \in \tilde{A}_{v, h}^{-}\left(4 \delta_{K}\right) .
\end{gathered}
$$


By (4-12), the first bound in (4-15), Holder's inequality, and a change of variables, we obtain

$$
\begin{aligned}
\left\|\left.D_{\widetilde{J}_{, v}}^{*} R_{v} \eta\right|_{\tilde{A}_{v, h}^{+}\left(\delta_{K}\right)}\right\|_{v, 1} & \leq C_{K}\left\|\left.d \tilde{u}_{v_{1}}\right|_{\tilde{q}_{0 ; 2}\left(\tilde{A}_{v, h}^{+}\left(\delta_{K}\right)\right.}\right\|\left\|_{v_{1}, p}\left|v_{h}\right|^{(p-2) / p}\right\| \eta \| \\
& \leq C_{K}^{\prime}\left|v_{h}\right|^{(p-1) / p} \sum_{i \in \chi(\mathcal{T}) \cap I_{h}}\left|\tilde{\rho}_{i}(v)\right| \cdot\|\eta\|,
\end{aligned}
$$

by (2b) of Corollary 3.8, since $\tilde{q}_{0 ; 2}\left(\tilde{A}_{v, h}^{+}\left(\delta_{K}\right)\right) \subset A_{v_{1}, h}^{-}\left(\left|v_{h}\right|\right)$. Similarly, by (4-13), (4-14), the second bound in (4-15), and Holder's inequality,

$$
\begin{aligned}
\left\|\left.D_{\widetilde{J}, v}^{*} R_{v} \eta\right|_{\tilde{A}_{v, h}^{-}\left(4 \delta_{K}\right)}\right\|_{v, 1} & \leq C_{K}\left(1+\left\|\left.d \tilde{u}_{v_{1}}\right|_{A_{v_{1}, h}^{-}\left(4 \delta_{K}\right)}\right\|_{v_{1}, p}\right)\left|v_{h}\right| \cdot\|\eta\| \\
& \leq C_{K}^{\prime}\left|v_{h}\right| \cdot\|\eta\|,
\end{aligned}
$$

by (2b) of Corollary 3.8. Since $D_{\widetilde{J}, v}^{*} R_{v} \eta$ is supported on the annuli $\widetilde{A}_{v, h}^{-}\left(\delta_{K}\right) \cup$ $\widetilde{A}_{v, h}^{+}\left(\delta_{K}\right)$, with $h \in I_{1}$, by (4-16) and (4-17),

$$
\left\|D_{\widetilde{J}, v}^{*} R_{v} \eta\right\|_{v, 1} \leq C_{K}|v|^{(p-1) / p}\|\eta\| .
$$

The last inequality in Lemma 4.4 is immediate from (4-18), since $p>2$.

We next prove the last estimate in (5) of Lemma 4.4. By the first inequalities in (2d) and (2e) of Corollary 3.8 , for all $\xi \in \widetilde{\Gamma}_{-}(b)$,

$$
\left|R_{v, \widetilde{J}} \xi\right|_{z} \leq C_{K} \frac{\left|v_{h}\right|}{|z|} \sum_{i \in \chi(\mathcal{T}) \cap I_{h}}\left|\tilde{\rho}_{i}(v)\right| \cdot\|\xi\|_{b, p, 1} \quad \forall z \in \tilde{A}_{v, h}^{+}\left(\delta_{K}\right) .
$$

By (4-12), the first bound in (4-15), (4-19), a change of variables, and Holder's inequality, we obtain

$$
\begin{aligned}
\left|\left\|R_{v, \tilde{J}} \xi,\left.D_{\widetilde{J}, v}^{*} R_{v} \eta\right|_{\tilde{A}_{v, h}^{+}\left(\delta_{K}\right)}\right\|_{v, 2}\right| \\
\leq C_{K}\left\|\left.d \tilde{u}_{v_{1}}\right|_{A_{v, h}^{-}\left(\left|v_{h}\right|\right)}\right\|_{v_{1}, p}\left|v_{h}\right|^{3 / 2-1 / p}\|\eta\|\|\xi\|_{b, p, 1} \\
\leq C_{K}^{\prime}\left|v_{h}\right|^{1 / 2} \sum_{i \in \chi(\mathcal{T}) \cap I_{h}}\left|\rho_{i}(v)\right| \cdot\|\eta\|\|\xi\|_{b, p, 1},
\end{aligned}
$$

by (2b) of Corollary 3.8. Since the map $\widetilde{q}_{0 ; 2}$ is holomorphic outside of the annuli $\widetilde{\mathcal{A}}_{b, h}^{ \pm}$ with $h \in I_{1}$ and $R_{v} \xi$ vanishes on $\tilde{\mathcal{A}}_{b, h}^{+}$,

$$
\left\langle D_{\widetilde{J}, v} R_{v, \widetilde{J}} \xi, R_{v} \eta\right\rangle_{v, 2}=\sum_{i \in I_{1}} \int_{\widetilde{\mathcal{A}}_{b, h}^{-}}\left\langle D_{\widetilde{J}_{, v}} R_{v} \xi, R_{v} \eta\right\rangle d z d \bar{z} .
$$


Since $\tilde{\mathcal{A}}_{b, h}^{-} \subset \tilde{A}_{v, h}^{+}\left(\delta_{K}\right)$, by (4-20) and integration by parts,

$$
\begin{aligned}
\left|\| D_{\widetilde{J}, v} R_{v, \widetilde{J}} \xi, R_{v} \eta\right\rangle+\sum_{h \in I_{1}} \oint_{\partial-\widetilde{\mathcal{A}}_{b, h}^{-}}\left\langle R_{v, \widetilde{J}} \xi,\right. & \left.\Pi_{\widetilde{\zeta}_{b ; v} \tilde{q}_{0 ; 2}(z)} \eta_{x_{h}(b)} \partial_{\bar{z}}\right\rangle d z \mid \\
& \leq C_{K}|v|^{1 / 2} \sum_{i \in \chi(\mathcal{T})}\left|\rho_{i}(v)\right| \cdot\|\eta\|\|\xi\|_{b, p, 1},
\end{aligned}
$$

Thus, by a change of variables and the definition of $R_{v, \tilde{J}}$,

$$
\begin{aligned}
\mid\left\langle D_{\widetilde{J}, v} R_{v, \widetilde{J}} \xi, R_{v} \eta\right\rangle-\sum_{h \in I_{1}} \oint_{\partial-A_{v_{1}, h}^{-}\left(\left|v_{h}\right| / \delta_{K}\right)}\left\langle R_{v_{1}, \widetilde{J} \xi, \Pi_{\widetilde{\zeta}_{b ; v}\left(w_{h}\right)} \eta_{x_{h}(b)} v_{h}\left|\frac{d w_{h}}{w_{h}^{2}}\right|}\right. & \leq C_{K}|v|^{1 / 2}|\rho(v)| \cdot\|\eta\|\|\xi\|_{b, p, 1},
\end{aligned}
$$

where $w_{h}=v_{h} / z$. The last estimate in (5) of Lemma 4.4 is immediate from (4-21) and the second estimate in $(2 \mathrm{~d})$ of Corollary 3.8 .

It remains to prove part (6) of Lemma 4.4. Let $\widetilde{\zeta}_{b ; v}: A_{v_{1}, h}^{-}\left(4 \delta_{K}\right) \longrightarrow T_{\operatorname{ev}_{P}(b)} X$ be as above. If $h \in I_{1}$ and $z \in \widetilde{\mathcal{A}}_{b, h}^{-}$,

$$
\begin{aligned}
\left|\Pi_{\widetilde{\zeta}_{b ; v}-\widetilde{q}_{v_{0} ; 2}(z)}^{\widetilde{\partial}-1} \circ \bar{\partial} \widetilde{J}_{u_{v}}-\bar{\partial}_{\widetilde{J}_{\mathrm{ev}_{P}(b)}}\left(\widetilde{\zeta}_{b ; v} \circ \widetilde{q}_{v_{0} ; 2}\right)\right|_{z} \\
\quad \leq C_{X}\left|\widetilde{\zeta}_{b ; v} \circ \widetilde{q}_{v_{0} ; 2}\right|_{z}\left|d\left(\widetilde{\zeta}_{b ; v} \circ \widetilde{q}_{v_{0} ; 2}\right)\right|_{z} ;
\end{aligned}
$$

see Section 2.3 of [14]. Thus, by integration by parts, if $\eta=Y \otimes d \bar{z}$,

$$
\begin{aligned}
\mid \int_{\widetilde{\mathcal{A}}_{b, h}^{-}}\left\langle\bar{\partial} \widetilde{J}_{v}, R_{v} \eta\right\rangle_{b} & -\oint_{\partial^{-} \widetilde{\mathcal{A}}_{b, h}^{-}}\left|\widetilde{\zeta}_{b ; v} \widetilde{q}_{v_{0} ; 2}(z), Y\right\rangle d z \mid \\
& \leq C_{X} \int_{\tilde{\mathcal{A}}_{b, h}^{-}}\left|\tilde{\zeta}_{b ; v} \circ \widetilde{q}_{v_{0} ; 2}\right| z\left|d\left(\tilde{\zeta}_{b ; v} \circ \widetilde{q}_{v_{0} ; 2}\right)\right|_{z} d z d \bar{z} \cdot\|\eta\|,
\end{aligned}
$$

since $\tilde{\zeta}_{b ; v}$ vanishes on $\Sigma_{v_{1}, \aleph}$. Since

$$
\oint_{\partial^{-} \tilde{\mathcal{A}}_{b, h}^{-}}\left\langle\tilde{\zeta}_{b ; v} \tilde{q}_{v_{0} ; 2}(z), Y\right\rangle d z=-\oint_{\partial^{-} A_{v_{1}, h}^{-}\left(\left|v_{h}\right|^{2} / \delta_{K}\right)}\left\langle\widetilde{\zeta}_{b ; v}\left(w_{h}\right), \eta_{x_{h}(b)}\left(v_{i}\right)\right\rangle \frac{d w_{h}}{w_{h}^{2}},
$$

where $w_{h}$ is as in the two previous paragraphs,

$$
\begin{aligned}
& \left|\oint_{\partial-\tilde{\mathcal{A}}_{b, h}^{-}}\left\langle\widetilde{\zeta}_{b ; v} \tilde{q}_{v_{0} ; 2}(z), Y\right\rangle d z+2 \pi \mathfrak{i} \sum_{i \in I_{h} \cap \chi(\mathcal{T})}\left\langle\mathcal{D}_{J, i} \rho_{i}(v), \eta_{x_{h}(b)}\right\rangle_{b}\right| \\
& \leq C_{K}\left(\|\widetilde{J}-J\|_{C^{1}}+|v|^{1 / p}+|v|^{(p-2) / p}\right) \sum_{i \in I_{h} \cap \chi(\mathcal{T})}\left|\rho_{i}(v)\right| \cdot\|\eta\|,
\end{aligned}
$$


by the two estimates in (2c) of Corollary 3.8. On the other hand, by Holder's inequality, change of variables, $(2 b)$ and the first estimate in (2c) of Corollary 3.8 ,

$$
\begin{aligned}
\int_{\tilde{\mathcal{A}}_{b, h}^{-}} \mid \tilde{\zeta}_{b ; v} & \left.\circ \tilde{q}_{v_{0} ; 2}|z| d\left(\tilde{\zeta}_{b ; v} \circ \tilde{q}_{v_{0} ; 2}\right)\right|_{z} d z d \bar{z} \\
& \leq C_{K}\left\|\tilde{\zeta}_{b ; v}\right\|_{C^{0}\left(\tilde{q}_{v_{0} ; 2}\left(\tilde{\mathcal{A}}_{b, h}^{-}\right)\right)} \cdot\left|v_{h}\right|^{(p-2) / p}\left\|\left.d \tilde{u}_{v_{1}}\right|_{\tilde{q}_{v_{0} ; 2}\left(\tilde{\mathcal{A}}_{b, h}^{-}\right)}\right\|_{v_{1}, p} \\
& \leq C_{K}^{\prime} \sum_{i \in I_{h} \cap \chi(\mathcal{T})}\left|\rho_{i}(v)\right|^{2}
\end{aligned}
$$

since $\tilde{q}_{v_{0} ; 2}\left(\tilde{\mathcal{A}}_{b, h}^{-}\right) \subset A_{v_{1}, h}^{-}\left(\left|v_{h}\right|^{2} / \delta_{K}\right)$. Since $\bar{\partial} \widetilde{J}_{v}$ is supported on the annuli $\tilde{\mathcal{A}}_{b, h}^{-}$, with $h \in I_{1}$, the estimate (6) of Lemma 4.4 follows from (4-23)-(4-25).

\subsection{Some geometric conclusions}

We now use the two-step gluing construction of the previous subsection to conclude the proof of Theorem 1.6.

Corollary 4.5 Suppose $(X, \omega, J), A \neq 0$, and $\mathcal{T}$ are as in Lemma 4.3 and $M=\varnothing$. If $J$ satisfies the regularity conditions (a) and (b-i), for every precompact open subset $K$ of $\mathcal{U}_{\mathcal{T}}(X ; J)$, there exist $\delta_{K}, C_{K} \in \mathbb{R}^{+}$and an open neighborhood $U_{K}$ of $K$ in $\mathfrak{X}_{1, \varnothing}(X, A)$ with the following properties:

(1) All requirements of Lemma 4.4 are satisfied.

(2) If $\tilde{J}$ is an almost complex structure on $X$ such that $\|\widetilde{J}-J\|_{C^{1}}<\delta_{K}$ and $[\tilde{b}] \in \mathfrak{M}_{1, \varnothing}^{0}(X, A ; \widetilde{J})$, the linearization $D_{\widetilde{J}, \tilde{b}}$ of $\bar{\partial}_{\widetilde{J}}$ at $\tilde{b}$ is surjective.

Proof (1) We continue with the notation preceding Lemma 4.4. By Lemma 4.4, it can be assumed that $\widetilde{b}=\left(\Sigma_{v}, j_{v}, \tilde{u}_{v}\right)$, where

$$
\tilde{u}_{v}=\exp _{u_{v}}^{\tilde{J}} \zeta_{\tilde{J}, v},\left.v \in \tilde{\mathcal{F}} \mathcal{T}_{\delta_{K}}^{\varnothing}\right|_{K^{(0)}}, \zeta_{\tilde{J}_{, v}} \in \Gamma_{+}(v),\left\|\zeta_{\tilde{J}_{, v}}\right\|_{v, p, 1} \leq \tilde{\delta}_{K},
$$

for some $\tilde{\delta}_{K} \in\left(0, \delta_{K}\right)$ to be chosen later. Since $\bar{\partial} \widetilde{J}_{v}=0$,

$$
\bar{\partial} \widetilde{J}_{v}+D_{\widetilde{J}, v} \zeta_{\widetilde{J}, v}+N_{\widetilde{J}, v} \zeta_{\widetilde{J}, v}=0
$$

where $N_{\widetilde{J}, v}$ is a quadratic term. In particular, $N_{\widetilde{J}, v} 0=0$ and

$$
\left\|N_{\widetilde{J}, v} \xi-N_{\widetilde{J}, v} \xi^{\prime}\right\|_{v, p} \leq C_{K}\left(\|\xi\|_{v, p, 1}+\left\|\xi^{\prime}\right\|_{v, p, 1}\right)\left\|\xi-\xi^{\prime}\right\|_{v, p, 1},
$$

if $\xi, \xi^{\prime} \in \Gamma(v)$ and $\|\xi\|_{v, p, 1},\|\xi\|_{v, p, 1}^{\prime} \leq \delta_{K}$. By (4-26), (4-27) and (3) of Lemma 4.4,

$$
\left\|\zeta_{\tilde{J}, v}\right\|_{v, p, 1} \leq C_{K}|\rho(v)|
$$

provided $\tilde{\delta}_{K}$ is sufficiently small. 
(2) Since $\tilde{u}_{v}$ is $\widetilde{J}$-holomorphic, all linearizations $D_{\widetilde{J}, \widetilde{u}}$ of $\bar{\partial} \widetilde{J}$ are the same. We give an explicit expression for $D_{\widetilde{J}, \widetilde{u}}$ and show that the dimension of its kernel does not exceed the index of $D_{\widetilde{J}, \tilde{u}}$. For any $\xi \in \Gamma\left(\Sigma_{v} ; \tilde{u}_{v}^{*} T X\right)$, let

$$
\widetilde{\xi}=\Pi_{\zeta_{\widetilde{J}, v}}^{\widetilde{J}-1} \xi
$$

We put

$$
\begin{aligned}
\bar{\partial} \widetilde{J} \xi & \equiv \Pi_{\zeta_{\widetilde{J}, v}}^{\widetilde{J}} \circ \Pi_{\widetilde{\xi}+\zeta_{\widetilde{J}, v}}^{\widetilde{J}-1} \circ \bar{\partial} \widetilde{J}_{\widetilde{J}} \exp _{u_{v}}^{\widetilde{J}}\left(\tilde{\xi}+\zeta_{\widetilde{J}, v}\right) \\
& =\Pi_{\zeta_{\widetilde{J}, v}}^{\widetilde{J}} \circ\left(\bar{\partial}_{\widetilde{J}} u_{v}+D_{\widetilde{J}, v}\left(\tilde{\xi}+\zeta_{\widetilde{J}, v}\right)+N_{\widetilde{J}, v}\left(\widetilde{\xi}+\zeta_{\widetilde{J}, v}\right)\right) \\
& =\Pi_{\zeta_{\widetilde{J}, v}}^{\widetilde{J}} \circ\left(D_{\widetilde{J}, v} \widetilde{\xi}+N_{\widetilde{J}, v}\left(\tilde{\xi}+\zeta_{\widetilde{J}, v}\right)-N_{\widetilde{J}, v} \zeta_{\widetilde{J}, v}\right),
\end{aligned}
$$

by (4-26). By (4-27), we can write

$$
N_{\widetilde{J}, v}\left(\tilde{\xi}+\zeta_{\widetilde{J}, v}\right)-N_{\widetilde{J}, v} \zeta_{\widetilde{J}, v}=L_{\widetilde{J}, v} \tilde{\xi}+\tilde{N}_{\widetilde{J}, v} \tilde{\xi}
$$

where $\tilde{N}_{\widetilde{J}, v}$ is a quadratic term, while the linear map $L_{\widetilde{J}, v}: \Gamma(v) \longrightarrow \Gamma^{0,1}(v ; \widetilde{J})$ satisfies

(4-29) $\left\|L_{\widetilde{J}, v} \xi\right\|_{v, p} \leq C_{K}\left\|\zeta_{\widetilde{J}, v}\right\|_{v, p, 1}\|\tilde{\xi}\|_{v, p, 1} \leq C_{K}^{\prime}|\rho(v)| \cdot\|\tilde{\xi}\|_{v, p, 1} \quad \forall \tilde{\xi} \in \Gamma(v)$, by (4-28). We conclude that

$$
D_{\widetilde{J}, \tilde{u}}=\Pi_{\zeta_{\widetilde{J}, v}} \circ\left(D_{\widetilde{J}, v}+L_{\widetilde{J}, v}\right) \circ \Pi_{\zeta_{\widetilde{J}, v}^{-1}} .
$$

Thus, it is sufficient to show that the dimension of the kernel of $D_{\widetilde{J}, v}+L_{\widetilde{J}, v}$ does not exceed the index of $D_{\widetilde{J}, v}$.

(3) Suppose $\xi \in \operatorname{ker}\left(D_{\widetilde{J}, v}+L_{\widetilde{J}, v}\right)$. Since the dimension of $\Gamma_{-}(v ; \widetilde{J})$ is the same as the dimension of $\Gamma_{-}(v)$, by (3) of Lemma 4.4, we can write

$$
\xi=\xi_{-}+\xi_{+}, \quad \text { where } \quad \xi_{-} \in \Gamma_{-}(v ; \widetilde{J}), \xi_{+} \in \Gamma_{+}(v) .
$$

If $\tilde{\delta}_{K}$ is sufficiently small, by (3) of Lemma 4.4 and (4-29),

$$
\begin{aligned}
\left\|\xi_{+}\right\|_{v, p, 1} & \leq C_{K}\left(\left\|D_{\widetilde{J}, v} \xi_{-}\right\|_{v, p}+\left\|L_{\widetilde{J}_{, v}} \xi_{-}\right\|_{v, p}\right) \\
& \leq C_{K}^{\prime}|v|^{(p-2) / p}\left\|\xi_{-}\right\|_{v, p, 1} \quad \forall \xi_{-}+\xi_{+} \in \operatorname{ker}\left(D_{\widetilde{J}, v}+L_{\widetilde{J}, v}\right) .
\end{aligned}
$$

Thus, the projection map, $\xi \longrightarrow \xi_{-}$is an injection on $\operatorname{ker}\left(D_{\widetilde{J}, v}+L_{\widetilde{J}, v}\right)$. We denote its image by $\Gamma_{-}^{*}(v ; \widetilde{J})$. Furthermore, by (4) of Lemma 4.4 and (4-29),

$$
\begin{aligned}
\left\|\xi_{+}\right\|_{v, p, 1} \leq C_{K}\left(\| D_{\widetilde{J}_{,}} \xi_{-}\right. & \left.\left\|_{v, p}+\right\| L_{\widetilde{J}_{, v}} \xi_{-} \|_{v, p}\right) \leq C_{K}^{\prime}|\rho(v)| \cdot\left\|\xi_{-}\right\|_{v, p, 1} \\
& \forall \xi_{-} \in \Gamma_{-}(v ; \widetilde{J}), \xi_{-}+\xi_{+} \in \operatorname{ker}\left(D_{\widetilde{J}, v}+L_{\widetilde{J}, v}\right) .
\end{aligned}
$$


(4) We now use Lemma 4.4 to estimate the $L^{2}$-inner product of

$$
\left\{D_{\widetilde{J}, v}+L_{\widetilde{J}, v}\right\}\left(\xi_{-}+\xi_{+}\right)
$$

with an element $R_{v} \eta$ of $\Gamma_{-}^{0,1}(v ; \widetilde{J})$, whenever

$$
\xi_{-} \in \widetilde{\Gamma}_{-}(v ; \widetilde{J}), \quad \xi_{+} \in \Gamma_{+}(v), \quad \xi_{-}+\xi_{+} \in \operatorname{ker}\left\{D_{\widetilde{J}_{, v}}+L_{\widetilde{J}_{, v}}\right\}
$$

By (4-11), (4-29) and (4-31),

$$
\mid\left\langle L_{\widetilde{J}_{, v}} \xi_{+}, R_{v} \eta\left\|\left.\left|\leq C_{K}\right| \rho(v)\right|^{2} \cdot\right\| \eta\|\| \xi_{-} \|_{v, p, 1}, \quad \forall \xi_{-} \in \widetilde{\Gamma}_{-}(v ; \widetilde{J}) .\right.
$$

By (4-31) and (7) of Lemma 4.4,

$$
\left|\left\langle D_{\widetilde{J}_{, v}} \xi_{+}, R_{v} \eta\right\rangle\right| \leq C_{K}|v|^{1 / 2}|\rho(v)| \cdot\|\eta\|\left\|\xi_{-}\right\|_{v, p, 1} \quad \forall \xi_{-} \in \tilde{\Gamma}_{-}(v ; \widetilde{J}) .
$$

For each $h \in I_{1}$, by (4-29) and (5) of Lemma 4.4,

$$
\begin{aligned}
\left|\left\langle\left. L_{\widetilde{J}_{, v}} \xi\right|_{\tilde{A}_{v ; h}^{-}\left(4 \delta_{K}\right)}, R_{v} \eta\right\rangle\right| & \leq C_{K}\left\|\left.\eta\right|_{\tilde{A}_{v ; h}^{-}\left(4 \delta_{K}\right)}\right\|_{v, 2}\left\|L_{\widetilde{J}_{, v}} \xi\right\|_{v, p} \\
& \leq C_{K}|v|^{1 / 2}|\rho(v)| \cdot\|\eta\|\|\xi\|_{v, p, 1} \quad \forall \xi \in \Gamma(v) .
\end{aligned}
$$

Since the metric $g_{v}$ on the annulus $\tilde{A}_{v ; h}^{+}\left(\delta_{K}\right)$ differs from the standard metric on the annulus with radii $2 \sqrt{\delta_{K}}$ and $\sqrt{\left|v_{h}\right|}$ by a factor bounded above by four and below by one-quarter,

$$
\|\xi\|_{C^{0}} \leq C_{K}\|\xi\|_{v, p, 1} \quad \forall \xi \in \Gamma\left(\tilde{A}_{v ; h}^{+}\left(\delta_{K}\right) ; u_{v}^{*} T X\right) ;
$$

see Section 3.1 in [14] and Section 3.3 in [16]. Thus,

$$
\begin{aligned}
\left\|\left.\left(L_{\widetilde{J}_{, v}} \xi\right)\right|_{\tilde{A}_{v ; h}^{+}\left(\delta_{K}\right)}\right\|_{v, p} & \leq C_{K}\left\|\zeta_{\widetilde{J}_{, v}}\right\|_{v, p, 1}\left\|\left.\xi\right|_{\tilde{A}_{v ; h}^{+}\left(\delta_{K}\right)}\right\|_{v, p, 1} \\
& \leq C_{K}^{\prime} \sum_{i \in \chi(\mathcal{T}) \cap I_{h}}\left|\rho_{i}(v)\right| \cdot\left\|\left.\xi\right|_{\tilde{A}_{v ; h}^{+}\left(\delta_{K}\right)}\right\|_{v, p, 1} \forall \xi \in \Gamma(v),
\end{aligned}
$$

since (4-27) is obtained from a pointwise bound; see Section 2.4 in [14]. By (4-29), (4-35) and (4) of Lemma 4.4,

$$
\begin{aligned}
\left|\| L_{\tilde{J}_{, v}} \xi_{-}\right| \tilde{A}_{v ; h}^{+}\left(\delta_{K}\right) & , R_{v} \eta\left\|\left|\leq C_{K}\|\eta\| \| \xi_{-}\right| \tilde{A}_{v ; h}^{+}\left(\delta_{K}\right)\right. \\
\leq C_{K}|v|^{1 / p} \sum_{i \in \chi(\mathcal{T}) \cap I_{h}}\left|\rho_{i}(v)\right| \cdot\|\eta\|\left\|\xi_{-}\right\|_{v, p, 1} & \forall \xi_{-} \in \tilde{\Gamma}_{-}(v ; \widetilde{J}) .
\end{aligned}
$$


Since the intersection of the support of $\xi_{-} \in \widetilde{\Gamma}_{-}(v ; \widetilde{J})$ with the support of $R_{v} \eta \in$ $\Gamma_{-}^{0,1}(v ; \widetilde{J})$ is contained in the $\left|I_{1}\right|$ annuli $\widetilde{A}_{v ; h}^{-}\left(4 \delta_{K}\right) \cup \widetilde{A}_{v ; h}^{+}\left(\delta_{K}\right)$, by (4-34) and (4-36),

$$
\begin{aligned}
||\left\langle L_{\widetilde{J}_{, v}} \xi_{-}, R_{v} \eta\right\rangle|| \leq C_{K}|v|^{1 / p}|\rho(v)| \cdot\|\eta\|\left\|\xi_{-}\right\|_{v, p, 1} \\
\quad \forall \eta \in \Gamma_{-}^{0,1}(b ; \widetilde{J}), \xi_{-} \in \widetilde{\Gamma}_{-}(v ; \widetilde{J}) .
\end{aligned}
$$

Finally, by (4-32), (4-33), (4-37) and (5) of Lemma 4.4,

$$
\begin{array}{r}
\mid\left\langle\left\{D_{\widetilde{J}, v}+L_{\widetilde{J}, v}\right\}\left(R_{v, \widetilde{J}} \xi+\xi_{+}\right), R_{v} \eta \|+2 \pi \mathfrak{i} \sum_{i \in \chi(\mathcal{T})}\left\langle\mathfrak{D}_{J, b ; i} \xi,\left.\eta_{x_{h(i)}(b)}\left(\rho_{i}(v)\right)\right|_{b}\right|\right. \\
\leq C_{K}\left(\|\widetilde{J}-J\|_{C^{1}}+|v|^{1 / p}+|v|^{(p-2) / p}\right)|\rho(v)| \cdot\|\eta\|\|\xi\|_{b, p, 1},
\end{array}
$$

for all $\xi \in \widetilde{\Gamma}_{-}(b)$ and $\xi_{+} \in \Gamma_{+}(v)$ such that $R_{v, \widetilde{J}} \xi+\xi_{+} \in \operatorname{ker}\left(D_{\widetilde{J}, v}+L_{\widetilde{J}, v}\right)$.

(5) Let $\left\{\eta_{r}\right\}$ be a basis for $\Gamma_{-}^{0,1}(b ; \widetilde{J})=\mathbb{E}_{\widetilde{\pi}_{P}(b)}^{*} \otimes \widetilde{J}_{\operatorname{ev}_{P}(b)} X$, orthonormal with respect to the inner-product corresponding to the norm $\|\cdot\|$. We define the homomorphism $\mathfrak{D}_{v}: \Gamma_{-}^{*}(v ; \widetilde{J}) \longrightarrow \Gamma_{-}^{0,1}(b ; \widetilde{J}) \quad$ by $\quad \mathfrak{D}_{v} \xi=\sum_{r}\left\|\left\{D_{\widetilde{J}, v}+L_{\widetilde{J}, v}\right\}\left(\xi+\xi_{+}\right), R_{v} \eta_{r}\right\| \eta_{r}$ if

$$
\xi_{+} \in \Gamma_{+}(v), \xi+\xi_{+} \in \operatorname{ker}\left(D_{\widetilde{J}_{, v}}+L_{\widetilde{J}, v}\right) .
$$

Since the projection map $\operatorname{ker}\left(D_{\widetilde{J}, v}+L_{\widetilde{J}, v}\right) \longrightarrow \Gamma_{-}^{*}(v ; \widetilde{J})$ is an isomorphism by (3) above, the map $\mathfrak{D}_{v}$ is well-defined. By definition, $\mathfrak{D}_{v} \equiv 0$. On the other hand, by (4-38),

(4-39) $\mathfrak{D}_{v} R_{v, \widetilde{J}} \xi=-2 \pi \mathfrak{i} \sum_{i \in \chi(\mathcal{T})}\left\{\mathfrak{D}_{J, b ; i}+\varepsilon_{i}(v)\right\} \xi \otimes \rho_{i}(v) \quad \forall \xi \in R_{v, \widetilde{J}}^{-1} \Gamma_{-}^{*}(v ; \widetilde{J})$ where $\varepsilon_{i}(v): R_{v, \widetilde{J}}^{-1} \Gamma_{-}^{*}(v ; \widetilde{J}) \longrightarrow T_{\operatorname{ev}_{P}(b)} X$ is a homomorphism such that

$$
\left|\varepsilon_{i}(v)\right| \leq\left. C_{K}\left(\|\widetilde{J}-J\|_{C^{1}}+|v|^{1 / p}+|v|^{(p-2) / p}\right) \quad \forall v \in \widetilde{\mathcal{F}} \mathcal{T}_{\delta_{K}}^{\varnothing}\right|_{K^{(0)}} .
$$

By (a) and (b-i) of Definition 1.4, the map

$$
\mathfrak{D}_{J, b ; i}: \widetilde{\Gamma}_{-}(b) \longrightarrow T_{\operatorname{ev}_{P}(b)} X
$$

is surjective for all $i \in \underset{\mathcal{F}}{\chi}(\mathcal{T})$; see the paragraph preceding Lemma 3.3. Since $\rho_{i}(v) \neq 0$

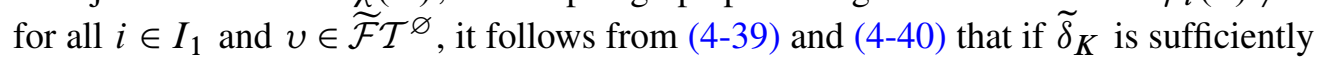


small,

$$
\begin{aligned}
\operatorname{dim} \operatorname{ker} D_{\widetilde{J}, \widetilde{u}} & =\operatorname{dim} \operatorname{ker}\left(D_{\widetilde{J}, v}+L_{\widetilde{J}, v}\right)=\operatorname{dim} \Gamma_{-}^{*}(v ; \widetilde{J})=\operatorname{dim} \operatorname{ker} \mathfrak{D}_{v} \\
& \leq \operatorname{dim} \Gamma_{-}(v ; \widetilde{J})-\operatorname{dim} \Gamma_{-}^{0,1}(b ; \widetilde{J})=\operatorname{dim} \Gamma_{-}(b)-\operatorname{dim} \Gamma_{-}^{0,1}(b ; J) \\
& =\operatorname{ind} D_{J, b}=\operatorname{ind} D_{\widetilde{J}, \widetilde{u}}
\end{aligned}
$$

as needed.

Corollary 4.5 concludes the proof of the genus-one regularity property of Theorem 1.6. Corollary 4.6 below and the Gromov compactness theorem imply that if $J$ is a genus-zero $A$-regular almost complex structure on $X, J_{r}$ is a sequence of almost complex structures on $X$ such that $J_{r} \longrightarrow J$ as $r \longrightarrow \infty$, and $b_{r} \in \mathfrak{M}_{1, M}^{0}\left(X, A ; J_{r}\right)$, then a subsequence of $\left\{b_{r}\right\}$ converges to an element of $\overline{\mathfrak{M}}_{1, M}^{0}(X, A ; J)$.

Corollary 4.6 If $(X, \omega, J), A \neq 0$, and $\mathcal{T}$ are as in Lemma 4.3, for every precompact open subset $K$ of $\mathcal{U}_{\mathcal{T}}(X ; J)-\mathcal{U}_{\mathcal{T} ; 1}(X ; J)$, there exist $\delta_{K} \in \mathbb{R}^{+}$and an open neighborhood $U_{K}$ of $K$ in $\mathfrak{X}_{1, M}(X, A)$ such that

$$
\mathfrak{M}_{1, M}^{0}(X, A ; \widetilde{J}) \cap U_{K}=\varnothing
$$

if $\tilde{J}$ is an almost complex structure on $X$ such that $\|\tilde{J}-J\|_{C^{1}}<\delta_{K}$.

Proof (1) Suppose $[\tilde{b}] \in \mathfrak{M}_{1, M}^{0}(X, A ; \widetilde{J}) \cap U_{K}$. By Lemma 4.4, it can be assumed that

$$
\widetilde{b}=\left(\Sigma_{v}, j_{v}, \tilde{u}_{v}\right)
$$

where $\quad \tilde{u}_{v}=\exp _{u_{v}}^{\tilde{J}} \zeta_{\widetilde{J}, v},\left.v \in \widetilde{\mathcal{F}} \mathcal{T}_{\delta_{K}}^{\varnothing}\right|_{K}(0), \zeta_{\widetilde{J}, v} \in \Gamma_{+}(v),\left\|\zeta_{\widetilde{J}, v}\right\|_{v, p, 1} \leq \tilde{\delta}_{K}$,

for some $\tilde{\delta}_{K} \in\left(0, \delta_{K}\right)$ to be chosen later. Since $\bar{\partial} \tilde{J}_{v}=0$,

$$
\bar{\partial} \widetilde{J}^{u}+D_{\widetilde{J}, v} \zeta_{\widetilde{J}, v}+N_{\widetilde{J}, v} \zeta_{\widetilde{J}, v}=0 .
$$

By (4-27) and (3) of Lemma 4.4,

$$
\left\|N_{\widetilde{J}, v} \zeta_{\widetilde{J}, v}\right\|_{v, p} \leq C_{K}\left\|\zeta_{\widetilde{J}, v}\right\|_{v, p, 1}^{2} \Longrightarrow\left\|\zeta_{\widetilde{J}, v}\right\|_{v, p, 1} \leq C_{K}|\rho(v)|,
$$

provided $\tilde{\delta}_{K}$ is sufficiently small.

(2) If $\eta \in \Gamma_{-}^{0,1}(b ; \widetilde{J})$, by (4-11), (4-42), and (7) of Lemma 4.4,

$$
\begin{aligned}
& \left.\left|\left\langle D_{\widetilde{J}_{, v}} \zeta_{\widetilde{J}, v}, R_{v} \eta\right\rangle\right|\left|\leq C_{K}\right| v\right|^{1 / 2}|\rho(v)| \cdot\|\eta\|, \\
& \left.\left|\left\langle N_{\widetilde{J}, v} \zeta_{\widetilde{J}, v}, R_{v} \eta\right\rangle\right|\left|\leq C_{K}\right| \rho(v)\right|^{2} \cdot\|\eta\| .
\end{aligned}
$$


By (4-41), (4-43), and (6) of Lemma 4.4, for all $\eta \in \Gamma_{-}^{0,1}(b ; \widetilde{J})$,

$$
\begin{aligned}
& \left|\sum_{i \in \chi(\mathcal{T})}\left\langle\mathcal{D}_{J, i} \rho_{i}(v), \eta_{x_{h(i)}(b)}\right\rangle_{b}\right| \\
& \leq C_{K}\left(\|\widetilde{J}-J\|_{C^{1}}+|v|^{1 / p}+|v|^{(p-2) / p}\right)|\rho(v)| \cdot\|\eta\| .
\end{aligned}
$$

On the other hand, since the closure of $K$ in $\mathcal{U}_{\mathcal{T}}(X ; J)-\mathcal{U}_{\mathcal{T} ; 1}(X ; J)$ is compact,

$$
\left|\sum_{i \in \chi(\mathcal{T})} \mathcal{D}_{J, i}\left(b, v_{i}\right)\right| \geq C_{K}^{-1}|v| \quad \forall b \in K^{(0)}, v=\left(v_{i}\right)_{i \in \chi(\mathcal{T})},
$$

for some $C_{K} \in \mathbb{R}^{+}$, by definition of the set $\mathcal{U}_{\mathcal{T} ; 1}(X ; J) \subset \mathcal{U}_{\mathcal{T}}(X ; J)$; see Theorem 2.3. Since $\Gamma_{-}^{0,1}(b ; \widetilde{J})=\mathbb{E}^{*} \otimes \operatorname{ev}_{P}^{*} T X,(4-44)$ and (4-45) imply that

$$
\|\widetilde{J}-J\|_{C^{1}}+|v|^{1 / p}+|v|^{(p-2) / p} \geq \widetilde{C}_{K}^{-1},
$$

as needed.

\section{Completion of proof of Theorem 1.2}

\subsection{Summary and setup}

In this section we sketch proofs of Propositions 5.1-5.3, based on the arguments of Sections 3 and 4. Detailed proofs of generalizations of these propositions can be found in [19, Section 5]. These three propositions are special cases of Theorem 1.2, but together they imply Theorem 1.2 for an arbitrary compact almost Kahler manifold $(X, \omega, J), J_{t}=J$ constant, and $A \in H_{2}(X ; \mathbb{Z})^{*}$. They also show that a limiting curve of a sequence of $J$-holomorphic curves in $X$ of arithmetic genus of at least one must have arithmetic genus of at least one as well, as is the case in algebraic geometry.

Suppose $\left\{b_{r}\right\}$ is a sequence of elements of $\overline{\mathfrak{M}}_{1, M}^{0}(X, A ; J)$ such that

$$
\lim _{r \longrightarrow \infty} b_{r}=b \in \overline{\mathfrak{M}}_{1, M}(X, A ; J)
$$

We need to show that $b \in \overline{\mathfrak{M}}_{1, M}^{0}(X, A ; J)$. By Definition 1.1, it is sufficient to assume that $b$ is an element of $\mathcal{U}_{\mathcal{T}}(X ; J)$ for a bubble type

$$
\mathcal{T}=(M, I, \aleph ; j, \underline{A})
$$

such that $A_{i}=0$ for all minimal elements $i \in I$. 
We can also assume that for some bubble type

$$
\mathcal{T}^{\prime}=\left(M, I^{\prime}, \aleph^{\prime} ; j^{\prime}, \underline{A^{\prime}}\right)
$$

$b_{r} \in \mathcal{U}_{\mathcal{T}^{\prime}}(X ; J)$ for all $r$. If $A_{i}^{\prime}=0$ for all minimal elements $i \in I^{\prime}$, the desired conclusion follows from Proposition 5.1 below, as it implies that the second condition in Definition 1.1 is closed with respect to the stable map topology. If $A_{i}^{\prime} \neq 0$ for some minimal element $i \in I^{\prime}$ and $\boldsymbol{\aleph}^{\prime} \neq \varnothing$, ie the principal component of $\Sigma_{b_{r}}$ is a circle of spheres, Proposition 5.2 implies that $b$ satisfies the second condition in Definition 1.1. Finally, if $\boldsymbol{\aleph}^{\prime}=\varnothing$ and $A_{i}^{\prime} \neq 0$ for the unique minimal element $i$ of $I^{\prime}$, the desired conclusion follows from Proposition 5.3.

We note that the three propositions are applied with $b$ and $b_{r}$ that are components of the ones above.

Proposition 5.1 Let $(X, \omega, J)$ be a compact almost Kahler manifold, $A \in H_{2}(X ; \mathbb{Z})^{*}$ and $M$ be a finite set. If $\left[b_{r}\right]$ is a sequence of elements in $\mathfrak{M}_{0,\{0\} \sqcup M}^{0}(X, A ; J)$ such that

$$
\lim _{r \longrightarrow \infty}\left[b_{r}\right]=[b] \in \mathcal{U}_{\mathcal{T}}(X ; J) \subset \overline{\mathfrak{M}}_{0,\{0\} \sqcup M}(X, A ; J),
$$

then either

(a) $\operatorname{dim}_{\mathbb{C}} \operatorname{Span}_{(\mathbb{C}, J)}\left\{\mathcal{D}_{i} b: i \in \chi(b)\right\}<|\chi(b)|$, or

(b) $\bigcap_{r=1}^{\infty} \overline{\bigcup_{r^{\prime}>r} \mathbb{C} \cdot \mathcal{D}_{\hat{0}} b_{r^{\prime}}} \subset \operatorname{Span}_{(\mathbb{C}, J)}\left\{\mathcal{D}_{i} b: i \in \chi(b)\right\}$.

Proposition 5.2 Let $(X, \omega, J)$ be a compact almost Kahler manifold, $M_{1}, \ldots, M_{n}$ be finite sets and

$$
n \in \mathbb{Z}^{+}, \quad A_{1}, \ldots, A_{n} \in H_{2}(X ; \mathbb{Z})^{*} .
$$

If $\left[b_{k, r}\right]$ is a sequence of elements in $\mathfrak{M}_{0,\{0,1\} \sqcup M_{k}}^{0}\left(X, A_{k} ; J\right)$ for each $k \in[n]$ such that

$$
\mathrm{ev}_{1}\left(b_{k, r}\right)=\mathrm{ev}_{0}\left(b_{k+1, r}\right) \quad \forall k \in[n-1], \quad \operatorname{ev}_{1}\left(b_{n, r}\right)=\operatorname{ev}_{0}\left(b_{1, r}\right),
$$

and

$$
\lim _{r \longrightarrow \infty}\left[b_{k, r}\right]=\left[b_{k}\right] \in \mathcal{U}_{\mathcal{T}^{(k)}}(X ; J) \subset \overline{\mathfrak{M}}_{0,\{0,1\} \sqcup M_{k}}\left(X, A_{k} ; J\right) \quad \forall k \in[n],
$$

where each $\mathcal{T}^{(k)} \equiv\left(\{1\} \sqcup M_{k}, I^{(k)} ; j^{(k)}, \underline{A}^{(k)}\right)$ is a bubble type such that $A_{i}^{(k)}=0$ for all $i \leq j_{1}$, then

$$
\operatorname{dim}_{\mathbb{C}} \operatorname{Span}_{(\mathbb{C}, J)}\left\{\mathcal{D}_{i} b_{k}: i \in \chi\left(b_{k}\right), k \in[n]\right\}<\sum_{k=1}^{k=n}\left|\chi\left(b_{k}\right)\right| .
$$


Proposition 5.3 Suppose $(X, \omega, J)$ is a compact almost Kahler manifold, $A \in$ $H_{2}(X ; \mathbb{Z})^{*}$, and $M$ is a finite set. If $\left[b_{r}\right]$ is a sequence of elements in $\mathfrak{M}_{1, M}^{0}(X, A ; J)$ such that

$$
\lim _{r \rightarrow \infty}\left[b_{r}\right]=[b] \in \mathcal{U}_{\mathcal{T}}(X ; J) \subset \overline{\mathfrak{M}}_{1, M}(X, A ; J),
$$

where $\mathcal{T} \equiv(M, I, \boldsymbol{\aleph} ; j, \underline{A})$ is a bubble type such that $A_{i}=0$ for all minimal elements $i \in I$, then $\operatorname{dim}_{\mathbb{C}} \operatorname{Span}_{(\mathbb{C}, J)}\left\{\mathcal{D}_{i} b: i \in \chi(b)\right\}<|\chi(b)|$.

We prove these three propositions in the next two subsections by combining the approach of Sections 3 and 4 with some aspects of the local setting used in [6]. The latter makes it possible to proceed with the genus-zero gluing construction of Section 3.1 and the first step of the genus-one gluing construction of Section 4.2 near a given bubble map $b$ even if $J$ is not genus-zero regular. The maps we encounter are not holomorphic on the entire domain, but are holomorphic on the parts of the domain that appear in Lemma 3.4. This is sufficient for the purposes of the key power series expansion in Lemma 3.5.

\subsection{Proofs of Propositions 5.1 and 5.2}

Let $(X, \omega, J), A, M, b_{r}$,

$$
b=(M, I ; x,(j, y), u),\left.\quad u_{i} \equiv u_{b}\right|_{\Sigma_{b, i}},
$$

and $\mathcal{T}$ be as in the statement of Proposition 5.1. We put

$$
I^{+}=\left\{i \in I: A_{i} \neq 0\right\} .
$$

For each $i \in I^{+}$, choose a finite-dimensional linear subspace

$$
\widetilde{\Gamma}_{-}^{0,1}(b ; i) \subset \Gamma\left(\Sigma_{b, i} \times X ; \Lambda_{J, j}^{0,1} \pi_{1}^{*} T^{*} \Sigma_{b, i} \otimes \pi_{2}^{*} T X\right)
$$

such that

$$
\begin{array}{r}
\Gamma\left(\Sigma_{b, i} ; \Lambda_{J, j}^{0,1} T^{*} \Sigma_{b, i} \otimes u_{i}^{*} T X\right)=\left\{D_{J, u_{b, i}} \xi: \xi \in \Gamma\left(\Sigma_{b, i} ; u_{i}^{*} T X\right), \xi(\infty)=0\right\} \\
\oplus\left\{\left\{\mathrm{id} \times u_{b, i}\right\}^{*} \eta: \eta \in \widetilde{\Gamma}_{-}^{0,1}(b ; i)\right\}
\end{array}
$$

and every element of $\widetilde{\Gamma}_{-}^{0,1}(b ; i)$ vanishes on a neighborhood of $\infty \in \Sigma_{b, i}$ and the nodes $x_{b, h} \in \Sigma_{b, i}$ with $\iota_{h}=i$. If $i \in I-I^{+}$, we denote by $\widetilde{\Gamma}_{-}^{0,1}(b ; i)$ the zero vector space. Let

$\tilde{\mathcal{U}}_{\mathcal{T}}(X ; J)=\left\{b^{\prime} \equiv\left(M, I ; x^{\prime},\left(j, y^{\prime}\right), u^{\prime}\right): b^{\prime}=\right.$ bubble map of type $\mathcal{T}$;

$$
\left.\bar{\partial}_{J, j} u_{i}^{\prime} \in\left\{\operatorname{id} \times u_{i}^{\prime}\right\}^{*} \widetilde{\Gamma}_{-}^{0,1}(b ; i) \forall i \in I\right\} .
$$


By the Implicit Function Theorem, $\tilde{\mathcal{U}}_{\mathcal{T}}(X ; J)$ is a smooth manifold near $b$. Let

$$
\widetilde{\mathcal{F}} \equiv \tilde{\mathcal{U}}_{\mathcal{T}}(X ; J) \times \mathbb{C}^{\widehat{I}}
$$

be the bundle of smoothing parameters.

Since the sequence $\left[b_{r}\right]$ converges to $[b]$, for all $r$ sufficiently large there exist

$$
b_{r}^{\prime} \in \tilde{\mathcal{U}}_{\mathcal{T}}(X ; J), \quad v_{r}=\left(b_{r}^{\prime}, v_{r}\right) \in \widetilde{\mathcal{F}}^{\varnothing} \quad \text { and } \quad \xi_{r} \in \Gamma\left(v_{r}\right) \equiv \Gamma\left(\Sigma_{v_{r}} ; u_{v_{r}}^{*} T X\right)
$$

such that

(5-1) $\quad \xi_{r}(\infty)=0 \forall r, \quad \lim _{r \longrightarrow \infty} b_{r}^{\prime}=b, \quad \lim _{r \rightarrow \infty}\left|v_{r}\right|=0, \quad \lim _{r \rightarrow \infty}\left\|\xi_{r}\right\|_{v_{r}, p, 1}=0$

and

$$
b_{r}=\exp _{b\left(v_{r}\right)} \xi_{r} \text {. }
$$

The last equality holds for a representative $b_{r}$ for $\left[b_{r}\right]$.

Remark The existence of $b_{r}^{\prime}, v_{r}$, and $\xi_{r}$ as above can be shown by an argument similar to the surjectivity argument in Section 4 of [16], with significant simplifications. In fact, the only facts about the bubble maps $b_{r}^{\prime}$ we use below are that they are constant on the degree-zero components and holomorphic on fixed neighborhoods of the attaching nodes of the first-level effective bubbles, ie on $\Sigma_{b_{r}^{\prime}}^{0}(\delta)$ in the notation of Section 3.3. Such bubble maps $b_{r}^{\prime}$, along with $v_{r}$ and $\xi_{r}$, can be constructed directly from the maps $b_{r}$; see the beginning of Section 4.4 in [16].

By the same argument as in the proofs of Lemma 3.5 and Corollary 3.6, but now applied to the sequence $\left(v_{r}, \xi_{r}\right)$ with sufficiently small $\delta_{K}$,

$$
\left|\mathcal{D}_{\hat{0}} b_{r}-\sum_{i \in \chi(\mathcal{T})}\left(\mathcal{D}_{i} b_{r}^{\prime}\right) \rho_{i}\left(v_{r}\right)\right| \leq C\left(\left|v_{r}\right|^{1 / p}+\left\|\xi_{r}\right\|_{v_{r}, p, 1}\right) \sum_{i \in \chi(\mathcal{T})}\left|\rho_{i}\left(v_{r}\right)\right| .
$$

This estimate follows from (3-18) with $b^{\prime}, v^{\prime}$ and $\zeta_{\tilde{J}, v^{\prime}}$ replaced by $b_{r}^{\prime}, v_{r}$, and $\xi_{r}$, respectively. Recall that $\Phi_{\widetilde{J}, v^{\prime}}(\infty)=$ id for $\widetilde{J}=J$. Since $b_{r}^{\prime} \longrightarrow b,(5-2)$ implies that

$$
\left|\mathcal{D}_{\hat{0}} b_{r}-\sum_{i \in \chi(\mathcal{T})}\left(\mathcal{D}_{i} b\right) \rho_{i}\left(v_{r}\right)\right| \leq C\left(\left|v_{r}\right|^{1 / p}+\left\|\xi_{r}\right\|_{v_{r}, p, 1}\right) \sum_{i \in \chi(\mathcal{T})}\left|\rho_{i}\left(v_{r}\right)\right|,
$$

where the difference is computed via a parallel transport of $T_{\mathrm{ev}_{0}\left(b_{r}^{\prime}\right)} X$ to $T_{\mathrm{ev}_{0}(b)} X$ with respect to the $J$-linear connection $\nabla^{J}$. By (5-1) and (5-3), $b$ must satisfy one of the two conditions in the statement of Proposition 5.1. 
The proof of Proposition 5.2 involves a similar extension of Lemma 3.5 and Corollary 3.7. By the assumption on the bubble types $\mathcal{T}^{(k)}$ made in Proposition $5.2, \mathrm{ev}_{0}\left(b_{k}\right)=$ $\mathrm{ev}_{1}\left(b_{k}\right)$ for all $k$. Thus,

$$
\mathrm{ev}_{1}\left(b_{k}\right)=\mathrm{ev}_{0}\left(b_{k}\right)=\mathrm{ev}_{1}\left(b_{l}\right) \quad \forall k, l \in[n] .
$$

Let $q$ denote the point $\operatorname{ev}_{0}\left(b_{1}\right)$. We identify a small neighborhood of $q$ in $X$ with a small neighborhood of $q$ in $T_{q} X$ via the exponential map of the metric $g_{X}$ and the tangent space to $X$ at a point close to $q$ with $T_{q} X$ via the $\nabla^{J}$-parallel transport.

For each pair $(k, r)$, with $r$ sufficiently large, let $\left(b_{k, r}^{\prime}, v_{k, r}, \xi_{k, r}\right)$ be an analogue of $\left(b_{r}^{\prime}, v_{r}, \xi_{r}\right)$ for $b_{k, r}$. As before, the key point is that the bubble maps $b_{k, r}^{\prime}$ are constant on the degree-zero components and holomorphic on fixed neighborhoods of the attaching nodes of the first-level effective bubbles. Let

$$
\zeta_{k, r}=\mathrm{ev}_{0}\left(b_{k, r}^{\prime}\right) \in T_{q} X
$$

and

$$
\widetilde{\zeta}_{k, r}=\mathrm{ev}_{1}\left(b_{k, r}\right)-\mathrm{ev}_{0}\left(b_{k, r}\right)=\mathrm{ev}_{1}\left(b_{k, r}\right)-\mathrm{ev}_{0}\left(b_{k, r}^{\prime}\right) \in T_{q} X .
$$

By the assumption on the maps $b_{k, r}$ made in the statement of Proposition 5.2,

$$
\begin{aligned}
\left|\zeta_{k, r}+\tilde{\zeta}_{k, r}-\zeta_{k+1, r}\right| & \leq C\left|\zeta_{k, r}\right| \cdot\left|\tilde{\zeta}_{k, r}\right| \\
\left|\zeta_{n, r}+\widetilde{\zeta}_{n, r}-\zeta_{1, r}\right| & \leq C\left|\zeta_{n, r}\right| \cdot\left|\tilde{\zeta}_{n, r}\right| ; \\
\Longrightarrow \quad\left|\tilde{\zeta}_{1, r}+\cdots+\widetilde{\zeta}_{n, r}\right| & \leq \epsilon_{r} \sum_{k=1}^{k=n}\left|\widetilde{\zeta}_{k, r}\right|
\end{aligned} \quad \forall k \in[n-1],
$$

for a sequence $\left\{\epsilon_{r}\right\}$ converging to 0 . On the other hand, by the proof of (3-25),

$$
\begin{aligned}
& \mid \tilde{\zeta}_{k, r}-\sum_{i \in \chi\left(\mathcal{T}^{(k)}\right)}\left(y_{1 ; i}\left(b_{k, r}^{\prime}\right)\right.\left.-x_{i ; 1}\left(b_{k, r}^{\prime}\right)\right)^{-1}\left(\mathcal{D}_{i} b_{k, r}^{\prime}\right) \rho_{i ; 1}\left(v_{k, r}\right) \mid \\
& \leq C\left(\left|v_{k, r}\right|^{1 / p}+\left\|\xi_{k, r}\right\|_{v_{k, r}, p, 1}\right) \sum_{i \in \chi\left(\mathcal{T}^{(k)}\right)}\left|\rho_{i ; 1}\left(v_{k, r}\right)\right|
\end{aligned}
$$

see (3) of the proof of Corollary 3.7 for notation. By (5-4) and (5-5),

$$
\begin{aligned}
\mid \sum_{k=1}^{k=n} \sum_{i \in \chi\left(\mathcal{T}^{(k)}\right)}\left(y_{1 ; i}\left(b_{k, r}^{\prime}\right)-x_{i ; 1}\left(b_{k, r}^{\prime}\right)\right)^{-1}\left(\mathcal{D}_{i} b_{k, r}^{\prime}\right) & \rho_{i ; 1}\left(v_{k, r}\right) \mid \\
& \leq \tilde{\epsilon}_{k} \sum_{k=1}^{k=n} \sum_{i \in \chi\left(\mathcal{T}^{(k)}\right)}\left|\rho_{i ; 1}\left(v_{k, r}\right)\right|,
\end{aligned}
$$

for a sequence $\left\{\tilde{\epsilon}_{r}\right\}$ converging to 0 . Since $\mathcal{D}_{i} b_{k, r}^{\prime} \longrightarrow \mathcal{D}_{i} b_{k}$ as $r \longrightarrow \infty$, (5-6) implies the conclusion of Proposition 5.2. 


\subsection{Proof of Proposition $\mathbf{5 . 3}$}

Let $(X, \omega, J), A, M, b_{r}$,

$$
b=(M, I, \boldsymbol{\aleph} ; S, x,(j, y), u),\left.\quad u_{i} \equiv u_{b}\right|_{\Sigma_{b, i}},
$$

and $\mathcal{T}$ be as in the statement of Proposition 5.3. Let $\mathcal{T}_{h}$ and $b_{h}$ for $h \in I_{1}$ be as in Section 2.2. For each $h \in I_{1}$ and $i \in I_{h}^{+}$, choose a subspace

$$
\widetilde{\Gamma}_{-}^{0,1}(b ; i) \equiv \widetilde{\Gamma}_{-}^{0,1}\left(b_{h} ; i\right) \subset \Gamma\left(\Sigma_{b, i} \times X ; \Lambda_{J, j}^{0,1} \pi_{1}^{*} T^{*} \Sigma_{b, i} \otimes \pi_{2}^{*} T X\right)
$$

as in the previous subsection. If $A_{i}=0$, denote by $\widetilde{\Gamma}_{-}^{0,1}(b ; i)$ the zero vector space. We define $\tilde{\mathcal{U}}_{\mathcal{T}}(X ; J)$ as at the beginning of Section 5.2. Let

$$
\widetilde{\mathcal{F} \mathcal{T}} \longrightarrow \tilde{\mathcal{U}}_{\mathcal{T}}(X ; J)
$$

be the bundle of gluing parameters. For each $b^{\prime} \in \tilde{\mathcal{U}}_{\mathcal{T}}(X ; J)$, let

$$
\widetilde{\Gamma}_{-}^{0,1}\left(b^{\prime}\right) \subset \Gamma\left(\Sigma_{b^{\prime}} \times X ; \Lambda_{J, j}^{0,1} \pi_{1}^{*} T^{*} \Sigma_{b^{\prime}} \otimes \pi_{2}^{*} T X\right)
$$

be the subspace obtained by extending all elements of $\widetilde{\Gamma}^{0,1}\left(b^{\prime} ; i\right)=\widetilde{\Gamma}^{0,1}(b ; i)$ by zero outside of the component $\Sigma_{b^{\prime}, i}$ of $\Sigma_{b^{\prime}}$.

The sequence $\left[b_{r}\right]$ converges to $[b]$. Thus, with notation as in Section 4.2, for all $r$ sufficiently large there exist

$$
b_{r}^{\prime} \in \tilde{\mathcal{U}}_{\mathcal{T}}(X ; J), \quad v_{r}=\left(b_{r}^{\prime}, v_{r}\right) \in \widetilde{\mathcal{F}}^{\varnothing}, \quad \xi_{r ; 1} \in \Gamma\left(v_{r ; 1}\right),
$$

and $\quad \xi_{r ; 2} \in \Gamma_{+}\left(v_{r}\right) \subset \Gamma\left(\tilde{u}_{v_{r ; 1}} \circ \widetilde{q}_{v_{r ; 0} ; 2}\right), \quad$ where $\quad \tilde{u}_{v_{r ; 1}}=\exp _{u_{v_{r} ; 1}} \xi_{r ; 1}$,

such that

$$
\begin{aligned}
& \left.\xi_{r ; 1}\right|_{\Sigma_{v_{r} ; 1} ; *}=0, \bar{\partial}_{J} \tilde{u}_{v_{r ; 1}} \in\left\{q_{v_{r ; 1}} \times \tilde{u}_{v_{r ; 1}}\right\}^{*} \widetilde{\Gamma}_{-}^{0,1}\left(b_{r}^{\prime}\right),
\end{aligned}
$$

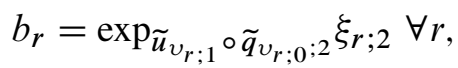

$$
\begin{aligned}
& \lim _{r \rightarrow \infty} b_{r}^{\prime}=b, \quad \lim _{r \rightarrow \infty}\left|v_{r}\right|=0, \\
& \lim _{r \longrightarrow \infty}\left\|\xi_{r ; 1}\right\|_{v_{r ; 1}, p, 1}=0, \quad \lim _{r \rightarrow \infty}\left\|\xi_{r ; 2}\right\|_{v_{r}, p, 1}=0 .
\end{aligned}
$$

We note that just as in the first step of the gluing construction in Section 4.2, there is no obstruction to smoothing the internal bubble nodes of the bubble map $b_{r}^{\prime}$ subject to the second condition in (5-7), as long as $b_{r}^{\prime}$ is sufficiently close to $b_{r}$. For defining the spaces $\Gamma_{+}\left(v_{r}\right)$ at the second step of the gluing construction, we take

$$
\Gamma_{-}\left(b^{\prime}\right)=\left\{\xi \in \Gamma\left(b^{\prime}\right): D_{J, b^{\prime}} \xi \in\left\{\operatorname{id} \times u_{b^{\prime}}\right\}^{*} \widetilde{\Gamma}_{-}^{0,1}\left(b^{\prime}\right)\right\} .
$$


The proof of the existence of the elements $v_{r}, \xi_{r ; 1}$, and $\xi_{r ; 2}$ as above is similar to the proof of the surjectivity property for the gluing map in Lemma 4.4, but simpler.

Since for each $h \in I_{1}$ the map $\tilde{u}_{v_{r ; 1}}$ is holomorphic on $\Sigma_{v_{r ; h}}^{0}(\delta) \subset \Sigma_{v_{r ; 1}}$ for $\delta \in \mathbb{R}^{+}$ sufficiently small, the estimates of Corollary 3.8 apply to each map $\tilde{u}_{v_{r ; 1}} \mid \Sigma_{v_{r ; 1}, h}$. Thus, we can define an obstruction bundle $\Gamma_{-}^{0,1}\left(v_{r}\right)$ for the second stage of the gluing construction in Section 4.2, with the estimates of Lemma 4.4 remaining valid. The claim of Proposition 5.3 is then obtained by the same argument as Corollary 4.6, with $\widetilde{J}, u_{v}$, and $\zeta_{\widetilde{J}, v}$ replaced by $J, \tilde{u}_{v_{r ; 1}} \circ \tilde{q}_{v_{r ; 0} ; 2}$, and $\xi_{r ; 2}$, respectively.

\section{Proof of Theorem 2.3}

\subsection{A multistep gluing construction}

The first part of the last claim of Theorem 2.3 can be proved by showing that a fine version of the converse to the $\widetilde{J}=J$ case of Corollary 4.6 holds. More precisely, using the two-step gluing construction of Section 4.2 and the Inverse Function Theorem twice, we can construct an orientation-preserving diffeomorphism

$$
\phi: \mathcal{F}^{1} \mathcal{T}_{\delta}^{\varnothing} \longrightarrow \mathfrak{M}_{1, k}^{0}(X, A ; J) \cap U_{\mathcal{T}} .
$$

Unfortunately, one of the families of the domain spaces involved in this construction does not extend continuously over $\mathcal{F}^{1} \mathcal{T}_{\delta} \cap \rho^{-1}(0)$ for a general bubble type $\mathcal{T}$ as in Theorem 2.3. As these domain spaces are needed to apply IFT over $\mathcal{F}^{1} \mathcal{T}_{\delta}-\mathcal{F}^{1} \mathcal{T}^{\varnothing}$, the above map $\phi$ cannot extend continuously over $\mathcal{F}^{1} \mathcal{T}_{\delta}$, except for bubble types $\mathcal{T}$ such that either $|\chi(\mathcal{T})|=1$ or $\chi(\mathcal{T})=\widehat{I}$. In the first case, both families do extend continuously over $\mathcal{F}^{1} \mathcal{T}$. In the second case, $\rho(v)=v$ for all $v \in \mathcal{F} \mathcal{T}$ and both families extend continuously over $\mathcal{F}^{1} \mathcal{T}-\{0\}$. On the other hand, as $v \longrightarrow 0$ both perturbations approach zero. This means that the corrections to be chosen in the domain spaces approach zero as well and thus extend continuously over $\mathcal{F}^{1} \mathcal{T}_{\delta}$.

In this subsection, we describe a multistep variation of the two-step gluing construction of Section 4.2. In the next subsection, we will use IFT multiple times to construct an orientation-preserving diffeomorphism

$$
\phi: \mathcal{F}^{1} \mathcal{T}_{\delta}^{\varnothing} \longrightarrow \mathfrak{M}_{1, k}^{0}(X, A ; J) \cap U_{\mathcal{T}} .
$$

Some of the domain spaces involved will not extend continuously over $\mathcal{F}^{1} \mathcal{T}_{\delta}$. However, whenever a domain space cannot be extended to a point $v^{*} \in \mathcal{F}^{1} \mathcal{T}_{\delta}$, the corresponding perturbations will approach zero as a sequence of elements $v_{r} \in \mathcal{F}^{1} \mathcal{T}_{\delta}^{\varnothing}$ approaches $v^{*}$. For this reason, the above diffeomorphism $\phi$ extends to a continuous map

$$
\phi: \mathcal{F}^{1} \mathcal{T}_{\delta} \longrightarrow \overline{\mathfrak{M}}_{1, k}^{0}(X, A ; J) \cap U_{\mathcal{T}}
$$


This map can shown to be a bijection by the same argument as in Section 4.1 of [16].

The multistep gluing construction described in this subsection is suitable for the purposes of Section 4.3 and thus could have been described in Section 4.2 instead of the two-stage gluing construction. However, describing the former in Section 4.3 would have further obscured the proofs of Corollaries 4.5 and 4.6. As these two corollaries appear far more central, than Theorem 2.3, to applications in the Gromov-Witten theory and enumerative geometry, we have postponed describing the multistep gluing construction until the present section.

If $b=\left(\Sigma_{b}, u_{b}\right)$ is any genus-one bubble map such that $\left.u_{b}\right|_{\Sigma_{b ; P}}$ is constant, let $\Sigma_{b}^{0} \subset \Sigma_{b}$ be the maximum connected union of the irreducible components of $\Sigma_{b}$ such that $\Sigma_{b ; P} \subset \Sigma_{b}^{0}$ and $\left.u_{b}\right|_{\Sigma_{b}^{0}}$ is constant. We put

$$
\Gamma_{B}(b)=\left\{\xi \in \Gamma\left(\Sigma_{b} ; u_{b}^{*} T X\right):\left.\xi\right|_{\Sigma_{b}^{0}}=0\right\}
$$

and

$$
\Gamma_{B}^{0,1}(b ; J)=\left\{\eta \in \Gamma\left(\Sigma_{b} ; \Lambda_{J, j}^{0,1} T^{*} \Sigma_{b} \otimes u_{b}^{*} T X\right):\left.\eta\right|_{\Sigma_{b}^{0}}=0\right\} .
$$

We denote by

$$
D_{J, b}^{B}: \Gamma_{B}(b) \longrightarrow \Gamma_{B}^{0,1}(b ; J)
$$

the restriction of the linearization $D_{J, b}$ of the $\bar{\partial}_{J}$-operator at $b$ defined with respect to the connection $\nabla^{X}$. Let

$$
\Gamma_{-}(b)=\operatorname{ker} D_{J, b} \quad \text { and } \quad \Gamma_{B ;-}(b)=\operatorname{ker} D_{J, b}^{B} .
$$

If $b$ is $J$-holomorphic, let $\widetilde{\Gamma}_{-}(b) \subset \Gamma_{B ;-}(b)$ be the subspace defined in Section 4.2; see (4-4) and (4-5).

Suppose $\mathcal{T}=(M, I, \boldsymbol{\aleph} ; j, \underline{A})$ is a bubble type as in Theorem 2.3 , ie $A_{i}=0$ for all $i \in I_{0}$, where $I_{0} \subset I$ is the subset of minimal elements. We put

$$
\begin{aligned}
& \chi^{0}(\mathcal{T})=\left\{h \in I: A_{i}=0 \forall i \leq h\right\}, \\
& \chi^{-}(\mathcal{T})=\{h \in \hat{I}: h<i \text { for some } i \in \chi(\mathcal{T})\} \subset \chi^{0}(\mathcal{T}), \\
& \langle\mathcal{T}\rangle=\max \{|\{h \in \hat{I}: h \leq i\}|: i \in \chi(\mathcal{T})\} \geq 1, \quad \mathcal{I}_{\langle\mathcal{T}\rangle}^{*}=\chi(\mathcal{T}) \text {, } \\
& \mathcal{I}_{\langle\mathcal{T}\rangle}=\hat{I}-\chi(\mathcal{T})-\chi^{-}(\mathcal{T})-I_{1},
\end{aligned}
$$

where $I_{1} \subset I$ is as in Section 2.2. For each $s \in\{0\} \cup[\langle\mathcal{T}\rangle-1]$, let

$$
\mathcal{I}_{s}=\left\{i \in \chi(\mathcal{T}) \cup \chi^{-}(\mathcal{T}):|\{h \in \hat{I}: h<i\}|=s\right\}, \quad \mathcal{I}_{s}^{*}=\mathcal{I}_{s} \cup \bigcup_{t=0}^{s-1}\left(\mathcal{I}_{t} \cap \chi(\mathcal{T})\right) .
$$


In the case of Figure 5,

$$
\langle\mathcal{T}\rangle=2, \quad \mathcal{I}_{0}=\left\{h_{1}, h_{3}\right\}, \quad \mathcal{I}_{1}=\left\{h_{4}, h_{5}\right\}, \quad \mathcal{I}_{2}=\left\{h_{2}\right\} .
$$

In general, the set $\mathcal{I}_{\langle\mathcal{T}\rangle}$ could be empty, but the sets $\mathcal{I}_{s}$ with $s<\langle\mathcal{T}\rangle$ never are.

If $b$ is a bubble map of type $\mathcal{T}$ as in Section 2.2 and $s \in[\langle\mathcal{T}\rangle]$, we put

$$
\Sigma_{b}^{(s)}=\bigcup_{i \in \chi^{0}(\mathcal{T})-\chi^{-}(\mathcal{T})}^{\bigcup \Sigma_{b, i}} \cup \bigcup_{h \in \mathcal{I}_{s-1}^{*}} \bigcup_{i<h} \Sigma_{b, i} \subset \Sigma_{b} .
$$

With notation as in Section 2.2, let

$$
\widetilde{\mathfrak{F} \mathcal{T}}=\bigoplus_{i \in \chi(\mathcal{T})} \overline{\mathcal{F}_{h(i)} \mathcal{T}} \longrightarrow \tilde{\mathcal{U}}_{\mathcal{T}}^{(0)}(X ; J)
$$

If $s \in[\langle\mathcal{T}\rangle]$ and $h \in \mathcal{I}_{s-1}^{*}$, let

$$
\chi_{h}(\mathcal{T})=\{i \in \chi(\mathcal{T}): h \leq i\}, \quad \widetilde{\mathfrak{F}_{h} \mathcal{T}}=\tilde{\mathcal{U}}_{\mathcal{T}}^{(0)}(X ; J) \times \mathbb{C}^{\chi_{h}(\mathcal{T})} .
$$

If in addition $v=(b, v) \in \widetilde{\mathcal{F} \mathcal{T}}$, let

$$
\begin{aligned}
\rho_{s ; h}(v) & =\left(b,\left(\rho_{h ; i}(v)\right)_{i \in \chi_{h}(\mathcal{T})}\right) \in \widetilde{\mathfrak{F}_{h} \mathcal{T}}, \quad \text { where } \quad \rho_{h ; i}(v)=\prod_{h<h^{\prime} \leq i} v_{h^{\prime}} \in \mathbb{C}, \\
\rho_{s}(v) & =\left(b,\left(\rho_{s ; h}(v)\right)_{h \in \mathcal{I}_{s-1}^{*}}\right) \in \widetilde{\mathfrak{F}_{s} \mathcal{T}} \equiv \underset{h \in \mathcal{I}_{s-1}^{*}}{\bigoplus \widetilde{\mathfrak{F}_{h} \mathcal{T}}}
\end{aligned}
$$

Note that $\rho(v) \in \widetilde{\mathfrak{F T}}$; see Section 2.2.

As in Section 4.2, for each $v=(b, v) \in \widetilde{\mathcal{F} \mathcal{T}}$ we put

$$
v_{0}=\left(b, v_{\aleph}, v_{0}\right) \text {. }
$$

Let $v_{\langle 0\rangle}=v$. If $s \in[\langle\mathcal{T}\rangle]$, let

$$
v_{s}=\left(b,\left(v_{h}\right)_{h \in \mathcal{I}_{s}}\right) \quad \text { and } \quad v_{\langle s\rangle}=\left(b,\left(v_{h}\right)_{h \in \mathcal{I}_{t}, t \geq s}\right) .
$$

The component $v_{\langle\mathcal{T}\rangle}$ of $v$ consists of smoothings at the nodes of $\Sigma_{b}$ that do not lie between the principal component $\Sigma_{b ; \kappa}$ of $\Sigma_{b}$ and the first-level effective bubbles and do not lie on $\Sigma_{b ; \kappa}$. These nodes will be smoothed out at the first step of the gluing construction, as specified by $v_{\langle\mathcal{T}\rangle}$. At the next step, we will smooth out the nodes indexed by the set $\mathcal{I}_{\langle\mathcal{T}\rangle-1}$, according the tuple of gluing parameters $v_{\langle\mathcal{T}\rangle-1}$. As in Section 4.2, at the last step we will smooth out, if possible, the nodes that lie on the principal component $\Sigma_{b ; \aleph}$ of $\Sigma_{b}$ according to $v_{0}$. This step will be obstructed. 
Suppose $v=(b, v) \in \widetilde{\mathcal{F} T}^{\varnothing}$ is a sufficiently small element. We will inductively construct approximately $J$-holomorphic bubble maps

$$
b_{s}(v)=\left(\Sigma_{v_{\langle s\rangle}}, u_{v, s}\right), \quad \forall s=0, \ldots,\langle\mathcal{T}\rangle,
$$

$J$-holomorphic bubble maps

$$
\tilde{b}_{s}(v)=\left(\Sigma_{v_{\langle s\rangle},} \tilde{u}_{v, s}\right), \quad \forall s=1, \ldots,\langle\mathcal{T}\rangle,
$$

and injective homomorphisms

$$
R_{v, s}: \Gamma_{-}(b) \longrightarrow \Gamma\left(\Sigma_{v_{\langle s\rangle} ;} ; u_{v, s}^{*} T X\right) \quad \text { and } \quad \tilde{R}_{v, s}: \Gamma_{-}(b) \longrightarrow \Gamma\left(\Sigma_{v_{\langle s\rangle}} ; \tilde{u}_{v, s}^{*} T X\right)
$$

such that the following properties are satisfied. First, for all $s \in[\langle\mathcal{T}\rangle]$,

$\Sigma_{b_{s}(v)}^{0}=\Sigma_{\widetilde{b}_{s}(v)}^{0}=q_{v_{\langle s\rangle}}^{-1}\left(\Sigma_{b}^{(s)}\right)$ and $u_{v, s}\left(\Sigma_{b_{s}(v)}^{0}\right)=\tilde{u}_{v, s}\left(\Sigma_{\widetilde{b}_{s}(v)}^{0}\right)=u_{b}\left(\Sigma_{b}^{0}\right) \equiv \operatorname{ev}_{P}(b)$,

where as before

$$
q_{v_{\langle s\rangle}}: \Sigma_{v_{\langle s\rangle}} \longrightarrow \Sigma_{b}
$$

is the basic gluing map of Section 2.2 in [16]. Second, for all $\xi \in \Gamma_{-}(b)$

$$
\begin{gathered}
\left.R_{v, s} \xi\right|_{\Sigma_{b_{s}(v)}^{0}},\left.\widetilde{R}_{v, s} \xi\right|_{\Sigma_{\widetilde{b}_{s}(v)}^{0}}=\text { const, } \\
R_{v, s} \xi\left(\Sigma_{b_{s}(v)}^{0}\right)=\widetilde{R}_{v, s} \xi\left(\Sigma_{\widetilde{b}_{s}(v)}^{0}\right)=\xi\left(\Sigma_{b}^{0}\right), \\
C(b)^{-1}\|\xi\|_{b, p, 1} \leq\left\|R_{v, s} \xi\right\|_{v_{\langle s\rangle}, p, 1},\left\|\widetilde{R}_{v, s} \xi\right\|_{v_{\langle s\rangle}, p, 1} \leq C(b)\|\xi\|_{b, p, 1}, \\
\left\|D_{J, b\left(v_{\langle s\rangle}\right)} R_{v, s} \xi\right\|_{v, p},\left\|D_{J, \tilde{b}\left(v_{\langle s\rangle}\right)} \widetilde{R}_{v, s} \xi\right\|_{v, p} \\
\leq C(b)\left(|v|^{1 / p}+|v|^{p-2 / p}\right)\|\xi\|_{b, p, 1},
\end{gathered}
$$

for some $C \in C\left(\mathcal{U}_{\mathcal{T}}(X ; J) ; \mathbb{R}^{+}\right)$.

Remark Similarly to Sections 3 and 4, above and below $\|\cdot\|_{v_{\langle s\rangle}, p, 1}$ denotes the weighted $L_{1}^{p}$-norms on the spaces

$$
\Gamma_{B}\left(\Sigma_{v_{\langle s\rangle}} ; u_{v, s}^{*} T X\right) \quad \text { and } \quad \Gamma_{B}\left(\Sigma_{v_{\langle s\rangle}} ; \tilde{u}_{v, s}^{*} T X\right)
$$

induced from the basic gluing map $q_{v_{\langle s\rangle}}$ as in Section 3.3 of [16]. Similarly, $\|\cdot\|_{v_{\langle s\rangle}, p}$ denotes the weighted $L^{p}$-norms on the spaces

$$
\Gamma_{B}\left(\Sigma_{v_{\langle s\rangle}} ; \Lambda_{J, j}^{0,1} T^{*} \Sigma_{v_{\langle s\rangle}} \otimes u_{v, s}^{*} T X\right) \quad \text { and } \quad \Gamma_{B}\left(\Sigma_{v_{\langle s\rangle}} ; \Lambda_{J, j}^{0,1} T^{*} \Sigma_{v_{\langle s\rangle}} \otimes \tilde{u}_{v, s}^{*} T X\right) .
$$

We denote the corresponding completions by $\Gamma_{B}\left(v_{\langle s\rangle}\right), \widetilde{\Gamma}_{B}\left(v_{\langle s\rangle}\right), \Gamma_{B}^{0,1}\left(v_{\langle s\rangle} ; J\right)$ and $\widetilde{\Gamma}_{B}^{0,1}\left(v_{\langle s\rangle} ; J\right)$. 
For $s \in\{0\} \cup[\langle\mathcal{T}\rangle]$, let $\Gamma_{-}\left(v_{\langle s\rangle}\right)$ be the image of $R_{v, s}$. Similarly, if $s \in[\langle\mathcal{T}\rangle]$, we denote by

$$
\Gamma_{B ;-}\left(v_{\langle s\rangle}\right) \subset \Gamma_{B}\left(b_{s}(v)\right) \quad \text { and } \quad \tilde{\Gamma}_{-}\left(v_{\langle s\rangle}\right), \tilde{\Gamma}_{B ;-}\left(v_{\langle s\rangle}\right) \subset \Gamma_{B}\left(\tilde{b}_{s}(v)\right)
$$

the image of $\Gamma_{B ;-}(b)$ under $R_{v, s}$, the image of $\widetilde{R}_{v, s}$, the image of $\Gamma_{B ;-}(b)$ under $\widetilde{R}_{v, s}$, respectively; see (6-1). Let $\Gamma_{B ;+}\left(v_{\langle s\rangle}\right)$ and $\widetilde{\Gamma}_{B ;+}\left(v_{\langle s\rangle}\right)$ be the $L^{2}$-orthogonal complements of $\Gamma_{B ;-}\left(v_{\langle s\rangle}\right)$ and $\widetilde{\Gamma}_{B ;-}\left(v_{\langle s\rangle}\right)$ in $\Gamma_{B}\left(v_{\langle s\rangle}\right)$ and $\widetilde{\Gamma}_{B}\left(v_{\langle s\rangle}\right)$. These spaces will satisfy

(6-4) $C(b)^{-1}\|\xi\|_{v_{\langle s\rangle}, p, 1} \leq\left\|D_{J, b\left(v_{\langle s\rangle}\right)} \xi\right\|_{v_{\langle s\rangle}, p} \leq C(b)\|\xi\|_{v_{\langle s\rangle}, p, 1} \forall \xi \in \Gamma_{B ;+}\left(v_{\langle s\rangle}\right)$,

(6-5) $C(b)^{-1}\|\xi\|_{v_{\langle s\rangle}, p, 1} \leq\left\|D_{J, \tilde{b}\left(v_{\langle s\rangle}\right)} \xi\right\|_{v_{\langle s\rangle}, p} \leq C(b)\|\xi\|_{v_{\langle s\rangle}, p, 1} \forall \xi \in \widetilde{\Gamma}_{B ;+}\left(v_{\langle s\rangle}\right)$.

Furthermore,

$$
\tilde{u}_{v, s}=\exp _{u_{v, s}} \zeta_{v, s}
$$

for some

$$
\zeta_{v, s} \in \Gamma_{B ;+}\left(v_{\langle s\rangle}\right) \text { such that }\left\|\zeta_{v, s}\right\|_{v_{\langle s\rangle}, p, 1} \leq C(b)|v|^{1 / p}
$$

Finally, for $\delta \in C\left(\mathcal{U}_{\mathcal{T}}(X ; J) ; \mathbb{R}^{+}\right)$sufficiently small, all maps

$$
v \longrightarrow b_{s}(v), \tilde{b}_{s}(v), \zeta_{v, s}, R_{v, \zeta}, \widetilde{R}_{v, \zeta}
$$

are smooth on $\widetilde{\mathcal{F}}_{\delta}^{\varnothing}$ and extend continuously over $\widetilde{\mathcal{F}}_{\delta}$.

We now describe the inductive construction referred to above. If $v \in \widetilde{\mathcal{F} \mathcal{T}} \varnothing$ is as above and $b=\left(\Sigma_{b}, u_{b}\right)$, we put

$$
u_{v,\langle\mathcal{T}\rangle}=u_{b} \circ q_{v_{\langle\mathcal{T}\rangle}}, \quad R_{v,\langle\mathcal{T}\rangle} \xi=\xi \circ q_{v_{\langle\mathcal{T}\rangle}} \quad \forall \xi \in \Gamma_{-}(b) .
$$

The first bounds in (6-3) and (6-4) with $s=\langle\mathcal{T}\rangle$ hold for the same reasons as the corresponding estimates in Lemma 3.1. Since the operator $D_{J, b}^{B}$ is surjective, by the first bound in (6-3) the operator

$$
D_{J, b_{\langle\mathcal{T}\rangle}(v)}^{B}: \Gamma_{B ;+}\left(v_{\langle\mathcal{T}\rangle}\right) \longrightarrow \Gamma_{B}^{0,1}\left(v_{\langle\mathcal{T}\rangle} ; J\right)
$$

is an isomorphism. On the other hand, by the construction of the map $q_{v,\langle\mathcal{T}\rangle}$ in Section 2.2 in [16],

$$
\left\|\bar{\partial}_{J} u_{v,\langle\mathcal{T}\rangle}\right\|_{v_{\langle\mathcal{T}\rangle}, p} \leq C(b)|v|^{1 / p} .
$$

Thus, by the Contraction Principle, if $v$ is sufficiently small, there exists a unique small element

$$
\zeta_{v,\langle\mathcal{T}\rangle} \in \Gamma_{B ;+}\left(v_{\langle\mathcal{T}\rangle}\right) \quad \text { such that } \quad \bar{\partial}_{J} \exp _{u_{v,\langle\mathcal{T}\rangle}} \zeta_{v,\langle\mathcal{T}\rangle}=0
$$


Furthermore, by (6-7),

$$
\left\|\zeta_{v,\langle\mathcal{T}\rangle}\right\|_{v_{\langle\mathcal{T}\rangle}, p, 1} \leq C(b)|v|^{1 / p}
$$

We thus define $\tilde{b}_{\langle\mathcal{T}\rangle}(v)$ by the first equation in (6-6).

If $s \in[\langle\mathcal{T}\rangle-1]$, let

$$
q_{v_{s} ;\langle\mathcal{T}\rangle+1-s}: \Sigma_{v_{\langle s\rangle}} \longrightarrow \Sigma_{v_{\langle s+1\rangle}}
$$

be the basic gluing map of Section 2.2 in [16] corresponding to the gluing parameter $v_{s}$. If $\widetilde{b}_{s+1}(v)$ and $\widetilde{R}_{v, s+1}$ have been defined, we put

$$
u_{v, s}=\tilde{u}_{v, s+1} \circ q_{v_{s} ;\langle\mathcal{T}\rangle+1-s}, \quad R_{v, s} \xi=\widetilde{R}_{v, s+1} \xi \circ q_{v_{s}} ;\langle\mathcal{T}\rangle+1-s \quad \forall \xi \in \Gamma_{-}(b) .
$$

The first bounds in (6-3) and (6-4) follow from the second estimates in (6-2) and (6-3) and from (6-5), with $s$ replaced by $s+1$. On the other hand, by the inductive construction and (6-6),

$$
\tilde{u}_{v, s+1}=\exp _{u_{v_{\langle s+1\rangle}}} \tilde{\zeta}_{v, s+1}
$$

for some

$$
\tilde{\zeta}_{v, s+1} \in \Gamma_{B}\left(\Sigma_{v_{\langle s+1\rangle}} ; u_{v_{\langle s+1\rangle}}^{*} T X\right)
$$

such that

$$
\left\|\tilde{\zeta}_{v, s+1}\right\|_{v_{\langle s+1\rangle}, p, 1} \leq C(b)|v|^{1 / p}
$$

where

$$
u_{v_{\langle s+1\rangle}}=u_{b} \circ q_{v_{\langle s+1\rangle}} .
$$

Thus, if $\delta$ is sufficiently small, the estimate in (2b) of Corollary 3.8 implies

$$
\left\|\left.d \tilde{u}_{v, s+1}\right|_{A_{v_{\langle s+1\rangle}, h}(\delta)}\right\|_{v, p} \leq C(b) \delta^{1 / p}\left|\rho_{s ; h}(v)\right| \quad \forall h \in \mathcal{I}_{s}^{*},
$$

where

$$
A_{v_{\langle s+1\rangle}, h}^{-}(\delta)=q_{v_{\langle s+1\rangle}}^{-1}\left(\left\{(h, z) \in \Sigma_{b, h}=\{h\} \times S^{2}:|z| \geq \delta^{-1 / 2} / 2\right\}\right) .
$$

It follows that

$$
\left\|\bar{\partial}_{J} u_{v, s}\right\|_{v, p} \leq C(b)|v|^{1 / p}\left|\rho_{s+1}(v)\right| \leq C(b)|v|^{1 / p} .
$$

Thus, similarly to the $s=\langle\mathcal{T}\rangle$ case above, if $v$ is sufficiently small, there exists a unique small element

$$
\zeta_{v, s} \in \Gamma_{B ;+}\left(v_{\langle s\rangle}\right) \quad \text { such that } \quad \bar{\partial}_{J} \exp _{u_{v, s}} \zeta_{v, s}=0 .
$$

Furthermore, by (6-11),

$$
\left\|\zeta_{v, s}\right\|_{v_{s}, p, 1} \leq C(b)|v|^{1 / p}
$$

We again define $\tilde{b}_{s}(v)$ by the first equation in (6-6). 
If $s \in[\langle\mathcal{T}\rangle]$ and $\tilde{b}_{s}(v)$ has been defined via (6-6), we put

$$
\widetilde{R}_{v, s} \xi=\Pi_{\zeta_{v, s}} R_{v, s} \xi \quad \forall \xi \in \Gamma_{-}(b),
$$

where $\Pi_{\zeta_{v, s}}$ is the parallel transport along the geodesics

$$
\tau \longrightarrow \exp _{u_{v, s}} \tau \zeta_{v, s}, \quad \tau \in[0,1]
$$

The bounds on $R_{v, s} \xi$ in (6-1)-(6-3) and the estimate (6-4), along with (6-6), imply the bounds on $\widetilde{R}_{v, s} \xi$ in (6-1)-(6-3) and the estimate (6-5).

At the final step of this inductive construction, we put

$$
\left.u_{v, 0}=\tilde{u}_{v, 1} \circ \tilde{q}_{v_{0} ;\langle\mathcal{T}\rangle+1}, \quad R_{v, 0} \xi \equiv \widetilde{R}_{v, 1} \xi \circ \widetilde{q}_{v_{0} ;\langle\mathcal{T}\rangle+1} \quad \forall \xi \in \Gamma_{-}(b)\right\},
$$

where

$$
\tilde{q}_{v_{0} ;\langle\mathcal{T}\rangle+1}: \Sigma_{v} \longrightarrow \Sigma_{v_{\langle 1\rangle}}
$$

is the modified basic gluing map constructed in Section 4.2 as $\tilde{q}_{v_{0} ; 2}$. In order to construct this map in this case, we need to replace $\delta_{K} \in \mathbb{R}^{+}$with $\delta \in C^{\infty}\left(\mathcal{U}_{\mathcal{T}}(X ; J)\right.$, which we view as a function on $\tilde{\mathcal{U}}_{\mathcal{T}}^{(0)}(X ; J)$ via the quotient projection map

$$
\tilde{\mathcal{U}}_{\mathcal{T}}^{(0)}(X ; J) \longrightarrow \mathcal{U}_{\mathcal{T}}^{(0)}(X ; J) .
$$

The homomorphism $R_{v, 0}$ satisfies the required properties. Let $\Gamma_{+}(v) \subset \Gamma(v)$ be the $L^{2}$-orthogonal complement of $\Gamma_{-}(v)$.

For each $h \in \widehat{I}$ and $\delta \in \overline{\mathbb{R}}^{+}$, let

$$
\begin{gathered}
A_{b, h}^{+}(\delta)=\left\{\left(\iota_{h}, z\right) \in \Sigma_{b, \iota_{h}}:\left|z-x_{h}(b)\right| \leq 2 \delta^{1 / 2}\right\}, \\
A_{b, h}^{-}(\delta)=\left\{(h, z) \in \Sigma_{b, h}=\{h\} \times S^{2}:|z| \geq \delta^{-1 / 2} / 2\right\}, \\
\Sigma_{b, h}^{*}(\delta)=\Sigma_{b, h}-A_{b, h}^{-}(\delta)-\bigcup_{\iota_{h^{\prime}}=h}^{+} A_{b, h^{\prime}}^{+}(\delta), \\
\widetilde{A}_{v, h}^{ \pm}(\delta)=q_{v}^{-1}\left(A_{b, h}^{ \pm}(\delta)\right) \subset \Sigma_{v}, \quad \widetilde{\Sigma}_{v, h}^{*}(\delta)=q_{v}^{-1}\left(\Sigma_{b, h}^{*}(\delta)\right) .
\end{gathered}
$$

We define the homomorphism

$$
R_{v}: \Gamma_{-}^{0,1}(b ; J) \longrightarrow \Gamma^{0,1}(v ; J)
$$

similarly to Section 4.2 , but with two changes. First, we replace the number $\delta_{K}$ with the function $\delta \in C^{\infty}\left(\mathcal{U}_{\mathcal{T}}(X ; J) ; \mathbb{R}^{+}\right)$. Second, we cut-off $R_{v}^{\aleph} \eta$ over the annuli

$$
\widetilde{A}_{v, i}^{+}(4 \delta(b))-\widetilde{A}_{v, i}^{+}(\delta(b))
$$

with $i \in \chi(\mathcal{T})$, instead of $h \in I_{1}$; see Section 2.2 in [13] for a version of this construction. Let $\Gamma_{-}^{0,1}(v ; J)$ be the image of $R_{v}$. We note that due to (6-9), the estimates in (3) and (6) of Lemma 4.4 remain valid. Of course, in this case $\widetilde{J}=J$ and $C_{K} \in \mathbb{R}^{+}$should 
be replaced by an $\mathbb{R}^{+}$-valued continuous function on $\mathcal{U}_{\mathcal{T}}(X ; J)$. We summarize the key results of this construction below.

Lemma 6.1 Suppose $(X, \omega, J)$ is a compact almost Kahler manifold, $A \in H_{2}(X ; \mathbb{Z})$, and $J$ is a genus-zero $A$-regular almost complex structure. If $\mathcal{T}=(M, I, \boldsymbol{\aleph} ; j, \underline{A})$ is a bubble type such that $\sum_{i \in I} A_{i}=A$ and $A_{i}=0$ for all minimal elements $i$ of $I$, there exist $\delta, C \in C\left(\mathcal{U}_{\mathcal{T}}(X ; J) ; \mathbb{R}^{+}\right)$and an open neighborhood $U_{\mathcal{T}}$ of $\mathcal{U}_{\mathcal{T}}(X ; J)$ in $\mathfrak{X}_{1, M}(X, A)$ such that

(1) $b_{s}(v), \tilde{b}_{s}(v), R_{v, s}$, and $\widetilde{R}_{v, s}$ as above are defined for all $v \in \widetilde{\mathcal{F}}_{\delta}^{\varnothing}$;

(2) for every $[\tilde{b}] \in \mathfrak{X}_{1, M}^{0}(X, A) \cap U_{K}$, there exist $v=(b, v) \in \tilde{\mathcal{F}} \mathcal{T}_{\delta}$ and $\zeta_{v, 0} \in \Gamma_{+}(v)$ such that

$$
\left\|\zeta_{v, 0}\right\|_{v, p, 1}<\delta(b) \quad \text { and } \quad\left[\exp _{b_{0}(v)} \zeta_{v, 0}\right]=[\tilde{b}] .
$$

Furthermore, such a pair $\left(v, \zeta_{v, 0}\right)$ is unique up to the $\operatorname{Aut}(\mathcal{T}) \propto\left(S^{1}\right)^{\hat{I}}$-action;

(3) for all $v=(b, v) \in \widetilde{\mathcal{F}}_{\delta}^{\varnothing}$,

$$
\begin{array}{rlrl}
\left\|\bar{\partial}_{J} u_{v, 0}\right\|_{v, p} & \leq C(b)|\rho(v)|, \\
\left\|D_{J, b_{0}}(v) \xi\right\|_{v, p} & \leq C(b)\left(|v|^{1 / p}+|v|^{(p-2) / p}\right)\|\xi\|_{v, p, 1} & \xi \in \Gamma_{-}(v), \\
C(b)^{-1}\|\xi\|_{v, p, 1} & \leq\left\|D_{J, b_{0}(v)} \xi\right\|_{v, p} \leq C(b)\|\xi\|_{v, p, 1} \quad \forall \xi \in \Gamma_{+}(v) ;
\end{array}
$$

(4) for all $v=(b, v) \in \widetilde{\mathcal{F}}_{\delta}^{\varnothing}, \xi \in \Gamma(v)$, and $\eta \in \Gamma_{-}^{0,1}(b ; J)$,

$$
\left.\left|\left\langle D_{J, b_{0}(v)} \xi, R_{v} \eta\right\rangle\right\rangle_{v, 2}|\leq C(b)| \rho(v)\right|^{1 / 2}\|\eta\|\|\xi\|_{v, p, 1} ;
$$

(5) for all $v=(b, v) \in \widetilde{\mathcal{F}}_{\delta}^{\varnothing}, s \in[\langle\mathcal{T}\rangle]$, and $h \in \mathcal{I}_{s-1}^{*}$,

$$
\left|\mathcal{D}_{h} \widetilde{b}_{s}(v)-\sum_{i \in \chi_{h}(\mathcal{T})} \mathcal{D}_{J, i} \rho_{h ; i}(v)\right| \leq C(b)|v|^{1 / p}\left|\rho_{s ; h}(v)\right| ;
$$

(6) for all $v=(b, v) \in \widetilde{\mathcal{F}}_{\delta}^{\varnothing}$ and $\eta \in \Gamma_{-}^{0,1}(b ; J)$,

$$
\begin{aligned}
& \mid\left\langle\bar{\partial}_{J} u_{v, 0}, R_{v} \eta\right\rangle+2 \pi \mathfrak{i} \sum_{i \in \chi(\mathcal{T})}\left\langle\mathcal{D}_{i} \rho_{i}(v),\left.\eta_{x_{h(i)}(b)}\right|_{b}\right| \\
& \quad \leq C(b)\left(|v|^{1 / p}+|v|^{(p-2) / p}\right)|\rho(v)| \cdot\|\eta\| .
\end{aligned}
$$

(7) all maps

$$
v \longrightarrow b_{s}(v), \tilde{b}_{s}(v), \zeta_{v, s}, R_{v, s}, \widetilde{R}_{v, s}
$$

are smooth on $\widetilde{\mathcal{F}}_{\delta}^{\varnothing}$ and extend continuously over $\widetilde{\mathcal{F} T}_{\delta}$. 
Due to (6-9), (5) is proved by the same argument as the $r=1$ case of the expansion (2a) in Lemma 3.5. Part (2) of Lemma 6.1 holds for the same reason as part (2) of Lemma 4.4. Regarding part (7) of Lemma 6.1, it is immediate from the inductive construction that each of the maps

$$
v \longrightarrow b_{s}(v), \tilde{b}_{s}(v), R_{v, s}, \tilde{R}_{v, s}
$$

is smooth on $\widetilde{\mathcal{F}}_{\delta}^{\varnothing}$ and extends continuously over $\widetilde{\mathcal{F}}_{\delta}$, provided this is the case for all the objects defined at the preceding steps of the construction. Under these circumstances the map $v \longrightarrow \zeta_{v, s}$ is also smooth, by the smooth dependence of the solutions of (6-8) and (6-12) on the parameters. It extends continuously over $\widetilde{\mathcal{F} \mathcal{T}} \delta$ by the same argument as in Section 4.1 in [16].

The estimate in (4) of Lemma 6.1 is an improvement on (7) of Lemma 4.4 and is proved by a similar argument. In this case, the support of $D_{J, v}^{*} R_{v} \eta$ is contained in the union of the annuli

$$
\begin{gathered}
\tilde{A}_{v, h}^{+}(\delta(b)), h \in \chi^{-}(\mathcal{T}) \cup \chi(\mathcal{T}) ; \quad \tilde{A}_{v, h}^{-}(\delta(b)), h \in \chi^{-}(\mathcal{T}) \cup \chi(\mathcal{T}) ; \\
\widetilde{\Sigma}_{v, h}^{*}(\delta(b)), h \in \chi^{-}(\mathcal{T}) ; \quad \tilde{A}_{v, h}^{-}(4 \delta(b))-\tilde{A}_{v, h}^{-}(\delta(b)), h \in \chi(\mathcal{T}) .
\end{gathered}
$$

Similarly to the proof of (7) of Lemma 4.4,

$$
\left|D_{J, v}^{*} R_{v} \eta\right|_{g_{v}, z} \leq C_{X}\left|d u_{v, 0}\right|_{g_{v}, z}|\eta|_{g_{v, z}}
$$

for every point $z$ of any of the annuli of the first three types above. Thus, the estimate (4-16) still applies to the annuli of the first type with $h \in I_{1}$. By definition of the metric $g_{v}$,

$$
\begin{aligned}
|\eta|_{g_{v, z}} \leq C(b)\|\eta\| \cdot \prod_{h^{\prime} \in \widehat{I}, h^{\prime}<h} v_{h^{\prime}} & \forall z \in \widetilde{A}_{v, h}^{+}(\delta(b)), \\
h \in \chi^{-}(\mathcal{T}) \cup \chi(\mathcal{T}) ; & \forall z \in \widetilde{\Sigma}_{v, h}^{*}(\delta(b)), \\
|\eta|_{g_{v, z}} \leq C(b)\|\eta\| \cdot \prod_{h^{\prime} \in \hat{I}, h^{\prime} \leq h} v_{h^{\prime}} & h \in \chi^{-}(\mathcal{T}) ; \\
|\eta|_{g_{v, z}} \leq C(b)\|\eta\| \cdot\left|w_{h}\right|^{-1} \prod_{h^{\prime} \in \widehat{I}, h^{\prime} \leq h} v_{h^{\prime}} \begin{array}{l}
\forall \\
z
\end{array} & h \in \widetilde{A}_{v, h}^{-}(4 \delta(b)),
\end{aligned}
$$

where $w_{h}$ is the coordinate on $\tilde{A}_{v, h}^{-}(4 \delta(b))$ defined similarly to $w_{i}$ in (2) of the proof of Lemma 3.5. On the other hand, by (6-10) and the assumption (a) of Definition 1.4,

$$
\left\|\zeta_{v, s}\left|\widetilde{\Sigma}_{v, i}^{*}(0) \|_{v_{\langle s\rangle}, p, 1} \leq C(b)\right| \rho_{s ; h}(v) \mid \quad \forall h \in \mathcal{I}_{s-1}^{*}, i \geq h ;\right.
$$


the above assumption implies that the operators $D_{J, b_{s}(v)}^{h}$ defined in Section 6.3 below are surjective. Note that by the inductive construction,

$$
\left.\zeta_{v, s}\right|_{\widetilde{\Sigma}_{v, i}^{*}(0)}=0 \quad \forall h \in \mathcal{I}_{s-1}^{*}, i<h .
$$

Combining this observation with (6-17), we find that

$$
\left\|\left.\widetilde{\zeta}_{v, 1}\right|_{\tilde{\Sigma}_{v, h}^{*}(0)}\right\|_{v_{\langle 1\rangle}, p, 1} \leq C(b)\left|\rho_{s ; h}(v)\right| \quad \forall h \in \widehat{I},
$$

where $\widetilde{\zeta}_{v, 1}$ is as in (6-9). Thus,

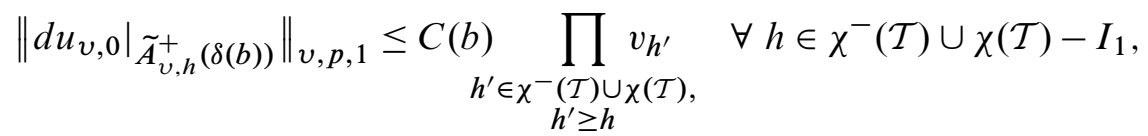

$$
\left\|\left.d u_{v, 0}\right|_{\tilde{\Sigma}_{v, h}^{*}(\delta(b))}\right\|_{v, p, 1} \leq C(b) \prod_{h^{\prime} \in \chi^{-}(\mathcal{T}) \cup \chi(\mathcal{T}),}^{h^{\prime}>h}, v_{h^{\prime}} \quad \forall h \in \chi^{-}(\mathcal{T}),
$$

$$
\left\|\left.d u_{v, 0}\right|_{\tilde{A}_{v, h}^{-}(4 \delta(b))}\right\|_{v, p, 1} \leq C(b) \prod_{\substack{h^{\prime} \in \chi^{-}(\mathcal{T}) \cup \chi(\mathcal{T}), h^{\prime}>h}} v_{h^{\prime}} \quad \forall h \in \chi^{-}(\mathcal{T}) \cup \chi(\mathcal{T}) .
$$

Combining (6-13)-(6-16), (6-18)-(6-20), and Holder's inequality, we find that the $L^{1}$-norm of $D_{J, v}^{*} R_{v} \eta$, with respect to the metric $g_{v}$, on each of the annuli above is bounded by $C(b)|\rho(v)|\|\eta\|$. Finally, analogously to (4-14),

$$
\begin{aligned}
\left|D_{J, v}^{*} R_{v} \eta\right|_{g_{v}, z} \leq C(b)\left(1+\left|d u_{v, 0}\right|_{g_{v}, z}\right)|\eta|_{g_{v, z}} \\
\forall z \in \widetilde{A}_{v, h}^{-}(4 \delta(b))-\widetilde{A}_{v, h}^{-}(\delta(b)), h \in \chi(\mathcal{T}) .
\end{aligned}
$$

Thus, by (6-16) and (6-20) the $L^{1}$-norm of $D_{J, v}^{*} R_{v} \eta$ on such annuli is also bounded by $C(b)|\rho(v)|\|\eta\|$.

Remark The exponent $1 / 2$ in (4) of Lemma 6.1 is due to the exponent $(p-1) / p$ in (4-16).

\subsection{Construction of diffeomorphism}

We continue with the notation of the previous subsection. For each $v=(b, v) \in \widetilde{\mathcal{F}}_{\delta}^{\varnothing}$, we define the homomorphism

$$
\pi_{v ;--}^{0,1}: \Gamma^{0,1}(v ; J) \longrightarrow \Gamma_{-}^{0,1}(b ; J) \quad \text { by } \quad \pi_{v ;-}^{0,1} \eta=\sum_{r}\left\langle\eta, R_{v} \eta_{r}\right\rangle \eta_{r} \in \Gamma_{-}^{0,1}(b ; J) \text {, }
$$


where $\left\{\eta_{r}\right\}$ is an orthonormal basis for $\Gamma_{-}^{0,1}(b ; J)$ as in (5) of the proof of Corollary 4.5. We denote the kernel of $\pi_{v ;-}^{0,1}$ by $\Gamma_{+}^{0,1}(v ; J)$. By Lemma 6.1,

$$
\begin{aligned}
U_{\mathcal{T}}^{\prime} & =\left\{\left[\exp _{b_{0}(v)} \zeta\right]: v=(b, v) \in \widetilde{\mathcal{F}} \mathcal{T}_{\delta}, \zeta \in \Gamma_{+}(v),\|\zeta\|_{v, p, 1}<\delta(b)\right\} \\
& \left.\subset\left\{\exp _{b_{0}(v)} \zeta\right]: v=(b, v) \in \widetilde{\mathcal{F}}_{\delta}, \zeta \in \Gamma_{+}(v),\|\zeta\|_{v, p, 1}<\delta(b)\right\}
\end{aligned}
$$

is an open neighborhood of $\mathcal{U}_{\mathcal{T}}(X ; J)$ in $\mathfrak{X}_{1, M}(X, A)$. Thus, we need to solve the equation

$(6-21) \bar{\partial}_{J} \exp _{u_{v, 0}} \zeta=0$

$$
\Longleftrightarrow\left\{\begin{array}{l}
\pi_{v ;-}^{0,1}\left(\bar{\partial}_{J} u_{v, 0}+D_{J, b_{0}(v)} \zeta+N_{v, 0} \zeta\right)=0 \in \Gamma_{-}^{0,1}(b ; J), \\
\bar{\partial}_{J} u_{v, 0}+D_{J, b_{0}(v)} \zeta+N_{v, 0} \zeta=0 \in \Gamma_{+}^{0,1}(v ; J),
\end{array}\right.
$$

where $N_{v, 0}$ is the quadratic term satisfying (4-27), for $v=(b, v) \in \widetilde{\mathcal{F}}_{\delta}^{\varnothing}$ and $\zeta \in$ $\Gamma_{+}(v)$ such that $\|\zeta\|_{v, p, 1}<\delta(b)$. By the proof of (1) of Corollary 4.6, there exist $\tilde{\delta}, \widetilde{C} \in C^{\infty}\left(\mathcal{U}_{\mathcal{T}}(X ; J) ; \mathbb{R}^{+}\right)$such that $\tilde{\delta}<\delta$ and every solution $(v, \zeta)$ of $(6-21)$

$$
|v|<\tilde{\delta}(b),\|\zeta\|_{v, p, 1}<\tilde{\delta}(b) \quad \Longrightarrow \quad\|\zeta\|_{v, p, 1}<\tilde{C}(b) \cdot|\rho(v)| .
$$

On the other hand, by (4) and (6) of Lemma 6.1 and (4-27),

$(6-23) \pi_{-}^{0,1} b_{0}(v, \zeta) \equiv \pi_{v ;-}^{0,1}\left(\bar{\partial}_{J} u_{v, 0}+D_{J, b_{0}(v)} \zeta+N_{v, 0} \zeta\right)=-2 \pi \mathfrak{i} \mathcal{D}_{\mathcal{T}}(v)+\varepsilon(v, \zeta)$

where $\mathcal{D}_{\mathcal{T}}$ is as defined in Section 2.2 and if

$$
\begin{gathered}
\|\varepsilon(v, \zeta)\| \leq C(b)\left(|v|^{1 / p}+|v|^{(p-2) / p}+\|\zeta\|_{v, p, 1}\right) \cdot\left(|\rho(v)|+\|\zeta\|_{v, p, 1}\right) \\
\zeta \in \Gamma(v),\|\zeta\|_{v, p, 1} \leq \delta(b) .
\end{gathered}
$$

We will first solve the top equation in (6-21) for $b^{\prime}=\mu_{0}(v, \zeta)$ and then use the Contraction Principle to show that the resulting bottom equation has a unique solution in $\zeta$ for each $v \in \widetilde{\mathcal{F}}^{1} \mathcal{T}_{\tilde{\delta}}^{\varnothing}$.

For each $s \in[\langle\mathcal{T}\rangle+1]$ and $h \in I_{1}$, let

$$
\begin{aligned}
\mathcal{U}_{\mathcal{T}_{h}}^{(s)}(X ; J)=\Psi_{\mathcal{T}}^{-1}\left(\left\{\left(0, r_{i}\right)_{i \in I_{h}} \in(\mathbb{C} \times \mathbb{R})^{I_{h}}: r_{i}=\frac{1}{2} \forall i \in I-\chi(\mathcal{T})\right.\right. \\
\left.\left.r_{i} \in\left(\frac{1}{2}-\frac{s}{4(\langle\mathcal{T}\rangle+1)}, \frac{1}{2}+\frac{s}{4(\langle\mathcal{T}\rangle+1)}\right) \forall i \in \chi_{h}(\mathcal{T})\right\}\right)
\end{aligned}
$$


see the end of Section 2.1. We put

$$
\begin{aligned}
& \mathcal{U}_{\mathcal{T}}^{(s)}(X ; J)=\left\{\left(b_{0},\left(b_{h}\right)_{h \in I_{1}}\right) \in \mathcal{U}_{\mathcal{T}_{0}}(X ; J) \times \prod_{h \in I_{1}} \mathcal{U}_{\mathcal{T}_{h}}^{(s)}(X ; J):\right. \\
&\left.\operatorname{ev}_{0}\left(b_{h}\right)=\operatorname{ev}_{\iota_{h}}\left(b_{0}\right) \forall h \in I_{1}\right\} \subset \tilde{\mathcal{U}}_{\mathcal{T}}^{(0)}(X ; J) ;
\end{aligned}
$$

see Section 2.2 for notation.

For each $s \in[\langle\mathcal{T}\rangle]$ and $h \in \mathcal{I}_{s-1}^{*}$, let

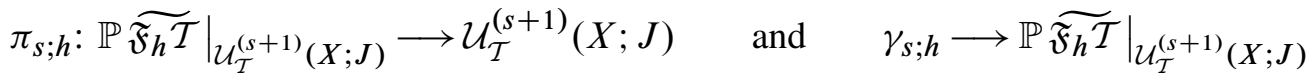

be the natural projection map and the tautological line bundle. With

$$
V_{s ; h}=\left.\pi_{s ; h}^{*} \operatorname{ev}_{P}^{*} T X \longrightarrow \mathbb{P} \widetilde{\mathfrak{F}_{h} \mathcal{T}}\right|_{\mathcal{U}_{\mathcal{T}}^{(s+1)}(X ; J)},
$$

we define

$$
\alpha_{s ; h} \in \Gamma\left(\left.\mathbb{P} \widetilde{\mathfrak{F}_{h} \mathcal{T}}\right|_{\mathcal{U}_{\mathcal{T}}^{(s+1)}(X ; J)} ; \gamma_{s ; h}^{*} \otimes V_{s ; h}\right)
$$

by $\alpha_{s ; h}\left(b,\left(v_{i}\right)_{i \in \chi_{h}(\mathcal{T})}\right)=\sum_{i \in \chi_{h}(\mathcal{T})} \mathcal{D}_{J, i}\left(b, v_{i}\right) \in T_{\operatorname{ev}_{P}(b)} X \quad$ if $\left(b,\left(v_{i}\right)_{i \in \chi_{h}(\mathcal{T})}\right) \in \gamma_{s ; h}$.

We denote by

$$
\pi_{s}: \mathbb{P}_{s} \mathcal{T} \equiv \prod_{h \in \mathcal{I}_{s-1}^{*}}\left(\mathbb{P} \widetilde{\mathfrak{F}_{h} \mathcal{T}}, \pi_{s ; h}\right) \longrightarrow \mathcal{U}_{\mathcal{T}}^{(s+1)}(X ; J)
$$

the fiber product of the bundles $\mathbb{P} \widetilde{\mathfrak{F}_{h} \mathcal{T}}$ over $\mathcal{U}_{\mathcal{T}}^{(s+1)}(X ; J)$. Let

$$
V_{s}=\pi_{s}^{*} \operatorname{ev}_{P}^{*} T X \longrightarrow \mathbb{P}_{s} \mathcal{T} \quad \text { and } \quad \gamma_{s}=\bigoplus_{h \in \mathcal{I}_{s-1}^{*}} \tilde{\pi}_{s ; h}^{*} \gamma_{s ; h},
$$

where $\tilde{\pi}_{s ; h}: \mathbb{P}_{s} \mathcal{T} \longrightarrow \mathbb{P} \widetilde{\mathfrak{F}_{h} \mathcal{T}}$ is the natural projection map. We denote by

$$
\alpha_{s} \in \Gamma\left(\mathbb{P}_{s} \mathcal{T} ; \gamma_{s}^{*} \otimes V_{s}\right)
$$

the section induced by the sections $\tilde{\pi}_{s ; h}^{*} \alpha_{s ; h}$ with $s \in \mathcal{I}_{s-1}^{*}$. Similarly, let $\pi_{0}: \mathbb{P}_{0} \mathcal{T}=\left.\mathbb{P} \widetilde{\mathfrak{F} \mathcal{T}}\right|_{\mathcal{U}_{\mathcal{T}}^{(1)}(X ; J)} \longrightarrow \mathcal{U}_{\mathcal{T}}^{(1)}(X ; J), \quad V_{0}=\pi_{0}^{*}\left(\tilde{\pi}_{P}^{*} \mathbb{E}^{*} \otimes \mathrm{ev}_{P}^{*} T X\right) \longrightarrow \mathbb{P}_{0} \mathcal{T}$ 
Let $\gamma_{0} \longrightarrow \mathbb{P}_{0} \mathcal{T}$ be the tautological line bundle. We define $\alpha_{0} \in \Gamma\left(\mathbb{P}_{0} \mathcal{T} ; \gamma_{0}^{*} \otimes V_{0}\right)$ by

$$
\left\{\alpha_{0}\left(b,\left(v_{i}\right)_{i \in \chi(b)}\right)\right\}(b, \psi)=-2 \pi \mathfrak{i} \sum_{i \in \chi(\mathcal{T})} \mathcal{D}_{J, i}\left(b, \psi_{x_{h(i)}(b)} v_{i}\right) \in T_{\operatorname{ev}_{P}(b)} X
$$

if

$$
\left(b,\left(v_{i}\right)_{i \in \chi(b)}\right) \in \gamma_{0}, \quad(b, \psi) \in \mathbb{E}_{\tilde{\pi}_{P}(b)} .
$$

Let

$$
\varphi: \tilde{\Gamma}_{-}(\cdot) \equiv \bigcup_{b \in \mathcal{U}_{\mathcal{T}}^{(\langle\mathcal{T}\rangle)}(X ; J)} \tilde{\Gamma}_{-}(b) \longrightarrow \tilde{\mathcal{U}}_{\mathcal{T}}^{(0)}(X ; J)
$$

be the smooth map induced from the maps $\varphi$ of the remark following Lemma 3.3 via the decomposition (2-11). In particular,

$$
\Sigma_{\varphi(b ; \xi)}=\Sigma_{b} \quad \text { and } \quad \operatorname{ev}_{P}(\varphi(b ; \xi))=\operatorname{ev}_{P}(b) \quad \forall b \in \mathcal{U}_{\mathcal{T}}^{(\langle\mathcal{T}\rangle)}(X ; J), \xi \in \widetilde{\Gamma}_{-}(b) .
$$

Thus, the fibers of the vector bundles

$\widetilde{\mathcal{F} \mathcal{T}}, \quad \tilde{\pi}_{P}^{*} \mathbb{E}^{*}, \quad \operatorname{ev}_{P}^{*} T X, \quad \widetilde{\mathfrak{F T}}$ and $\widetilde{\mathfrak{F}_{h} \mathcal{T}}$ for $h \in \mathcal{I}_{s-1}^{*}, s \in[\langle\mathcal{T}\rangle]$, at $b$ and at $\varphi(b, \xi)$ are canonically isomorphic. If $\xi \in \widetilde{\Gamma}_{-}(b)$ is sufficiently small,

$$
v \equiv(b, v) \in \widetilde{\mathcal{F}}_{\delta}^{\varnothing} \quad \text { and } \quad v^{\prime}=(\varphi(b, \xi), v) \in \widetilde{\mathcal{F}}_{\delta}^{\varnothing}
$$

are corresponding elements of the fibers of $\widetilde{\mathcal{F} \mathcal{T}}$ at $b$ and at $\varphi(b, \xi)$, let $\zeta_{v, 0}(\xi) \in \Gamma(v)$ be given by

$$
\exp _{u_{v, 0}} \zeta_{v, 0}(\xi)=u_{v^{\prime}, 0} \quad \text { and } \quad\left\|u_{v, 0}\right\|_{C^{0}}<r_{J}
$$

We identify $\Gamma_{+}(v)$ and $\Gamma_{+}^{0,1}(v ; J)$ with $\Gamma_{+}\left(v^{\prime}\right)$ and $\Gamma_{+}^{0,1}\left(v^{\prime} ; J\right)$ by composing the $\nabla^{J}$-parallel transports

$$
\Gamma_{+}(v) \longrightarrow \Gamma\left(v^{\prime}\right) \quad \text { and } \quad \Gamma_{+}^{0,1}(v ; J) \longrightarrow \Gamma^{0,1}\left(v^{\prime} ; J\right)
$$

along the geodesics corresponding to $\zeta_{v, 0}(\xi)$ with $L^{2}$-projection maps

$$
\Gamma\left(v^{\prime}\right) \longrightarrow \Gamma_{+}\left(v^{\prime}\right) \quad \text { and } \quad \Gamma^{0,1}\left(v^{\prime} ; J\right) \longrightarrow \Gamma_{+}^{0,1}\left(v^{\prime} ; J\right)
$$

corresponding to the metric $g_{v}=g_{v^{\prime}}$ on $\Sigma_{v}$.

For each $s \in\{0\} \cup[\langle\mathcal{T}\rangle]$, the map $\varphi$ induces a smooth map

$$
\varphi_{s}:\left.\mathfrak{G}_{s} \equiv \pi_{s}^{*} \widetilde{\Gamma}_{-}(\cdot)\right|_{\mathcal{U}_{\mathcal{T}}^{(s)}(X ; J)} \longrightarrow \mathbb{P}_{s} \mathcal{T} .
$$

Similarly to the previous paragraph, the fibers of vector bundles

$$
\pi_{s}^{*} \widetilde{\mathcal{F} \mathcal{T}}, \quad \gamma_{s} \text { and } V_{s}
$$


at $\tilde{b}$ and $\varphi_{s}(\tilde{b}, \xi)$ are canonically isomorphic, if $(b, \xi) \in \mathfrak{G}_{s}$ is sufficiently small. By the regularity assumptions (a) and (b-i) of Definition 1.4, the differential

$$
\nabla^{\mathcal{T}} \alpha_{s}: \mathfrak{G}_{s} \longrightarrow \gamma_{s}^{*} \otimes V_{s}
$$

of $\alpha_{s}$, defined via the above isomorphisms, is surjective. Let $\mathfrak{G}_{s}^{\perp}$ be the $L^{2}$-orthogonal complement of $\operatorname{ker} \nabla^{\mathcal{T}} \alpha_{s}$ in $\mathfrak{G}_{s}$. By the surjectivity of $\nabla^{\mathcal{T}} \alpha_{s}$, the Contraction Principle, the precompactness of the fibers of

$$
\pi \circ \pi_{s}: \mathbb{P}_{s} \mathcal{T} \longrightarrow \mathcal{U}_{\mathcal{T}}^{(s+1)}(X ; J) \longrightarrow \mathcal{U}_{\mathcal{T}}(X ; J),
$$

there exists $\epsilon, C \in C\left(\mathcal{U}_{\mathcal{T}}(X ; J) ; \mathbb{R}^{+}\right)$with the following property. If $b \in \mathcal{U}_{\mathcal{T}}(X ; J)$ and

$$
\kappa \in \Gamma\left(\left.\mathbb{P}_{s} \mathcal{T}\right|_{\pi^{-1}(b)} ; \gamma_{s}^{*} \otimes V_{s}\right)
$$

is a smooth section such that

$$
\|\kappa(\tilde{b})\|,\left\|\nabla^{\mathcal{T}} \kappa(\tilde{b})\right\| \leq\left.\epsilon(b) \quad \forall \tilde{b} \in \mathbb{P}_{s} \mathcal{T}\right|_{\pi^{-1}(b)},
$$

then for every $\left.\tilde{b}^{*} \in \mathbb{P}_{S} \mathcal{T}\right|_{\pi^{-1}(b) \cap \mathcal{U}_{\mathcal{T}}^{(s)}(X ; J)}$ the equation

$\alpha_{s}\left(\varphi_{s}\left(\tilde{b}^{*}, \xi\right)\right)+\kappa\left(\varphi_{s}\left(\tilde{b}^{*}, \xi\right)\right)=\alpha_{s}\left(b^{*}\right) \in \gamma_{s}^{*} \otimes V_{s},\left.\quad \xi \in \mathfrak{G}_{s}\right|_{\tilde{b}^{*}},|\xi|<2 C(b) \epsilon(b)$,

has a unique solution $\xi_{\kappa}\left(\widetilde{b}^{*}\right)$. Furthermore,

$$
\left|\xi_{\kappa}\left(\tilde{b}^{*}\right)\right| \leq 2 C(b) \max \left\{\|\kappa(\tilde{b})\|,\left\|\nabla^{\mathcal{T}} \kappa(\tilde{b})\right\|:\left.\tilde{b} \in \mathbb{P}_{S} \mathcal{T}\right|_{\pi^{-1}(b)}\right\} ;
$$

see Section 3.6 in [16], for example.

We are now ready to return to the gluing construction of the previous subsection. For every element $v=(b, v)$ of $\widetilde{\mathcal{F}}_{\delta}^{\varnothing}$, let

$$
\mu_{\langle\mathcal{T}\rangle+1}(v)=b \in \tilde{\mathcal{U}}_{\mathcal{T}}^{(0)}(X ; J) \quad \text { and } \quad \tilde{\mu}_{\langle\mathcal{T}\rangle+1}(v)=\left(\mu_{\langle\mathcal{T}\rangle+1}(v), v\right) \in \widetilde{\mathcal{F}}_{\delta}^{\varnothing} .
$$

Suppose $s \in[\langle\mathcal{T}\rangle]$ and for all $t \in[\langle\mathcal{T}\rangle]$ such that $t>s$ and $\left.v \in \widetilde{\mathcal{F}}_{\delta}^{\varnothing}\right|_{\mathcal{U}_{\mathcal{T}}^{(t)}(X ; J)}$ as above we have constructed

$$
\mu_{t}(v) \in \tilde{\mathcal{U}}_{\mathcal{T}}^{(0)}(X ; J) \quad \text { and } \quad \tilde{\mu}_{t}(v)=\left(\mu_{t}(v), v\right) \in \widetilde{\mathcal{F}}_{\delta}^{\varnothing}
$$

such that

$$
\begin{gathered}
\mathcal{D}_{h} \tilde{b}_{t}\left(\tilde{\mu}_{t}(v)\right)=\left\{\alpha_{t ; h}(b)\right\}\left(\rho_{t ; h}(v)\right) \quad \forall h \in \mathcal{I}_{t-1}^{*}, \\
\mu_{t}(v)=\mu_{t+1}\left(\varphi\left(b, \xi_{v, t}\right), v\right) \quad \text { for some } \quad \xi_{v, t} \in \widetilde{\Gamma}_{-}(b) \\
\text { such that } \quad\left|\xi_{v, t}\right|,\left|\nabla^{\mathcal{T}} \xi_{v, t}\right| \leq C(b)|v|^{1 / p},\left.\left(\left[\rho_{t}(v)\right], \xi_{v, t}\right) \in \mathfrak{G}_{t}^{\perp}\right|_{\left[\rho_{t}(v)\right]},
\end{gathered}
$$


where $\left[\rho_{t}(v)\right] \in \mathbb{P}_{t} \mathcal{T}$ denotes the image of $\rho_{t}(v) \in \widetilde{\mathfrak{F}_{t} \mathcal{T}^{\varnothing}}$ under the projection map $\widetilde{\mathfrak{F}_{t} \mathcal{T}}{ }^{\varnothing} \longrightarrow \mathbb{P}_{t} \mathcal{T}$ and $\nabla^{\mathcal{T}} \xi_{v, t}$ is the covariant derivative of $\xi_{v, t}$ along the directions in $\widetilde{\Gamma}_{-}(b)$ as before.

By (5) of Lemma 6.1, its proof and (6-26),

$$
\mathcal{D}_{h} \widetilde{b}_{s}\left(\tilde{\mu}_{s+1}(v)\right)=\left\{\alpha_{s ; h}(b)+\varepsilon_{s ; h}(v)\right\}\left(\rho_{s ; h}(v)\right) \quad \forall h \in \mathcal{I}_{s-1}^{*},
$$

where $\varepsilon_{s ; h} \in T_{\mathrm{ev}_{P}(b)} X$ satisfies

$$
\left|\varepsilon_{s ; h}(v)\right|,\left|\nabla^{\mathcal{T}} \varepsilon_{s ; h}(v)\right| \leq C(b)|v|^{1 / p} .
$$

The estimate (6-27) can be restated as

$$
\left(\mathcal{D}_{h} \tilde{b}_{s}\left(\tilde{\mu}_{s+1}(v)\right)\right)_{h \in \mathcal{I}_{s-1}^{*}}=\left\{\alpha_{s}\left(\left[\rho_{s}(v)\right]\right)+\varepsilon_{s}(v)\right\}\left(\rho_{s}(v)\right),
$$

where $\left.\varepsilon_{s}(v) \in \gamma_{s}^{*} \otimes V_{s}\right|_{\left[\rho_{s}(v)\right]}$ satisfies the analogue of (6-28). Thus, by the previous paragraph, (6-27), and (6-28), there exists a unique small element $\xi_{v, s} \in \widetilde{\Gamma}_{-}(b)$ such that

$$
\left.\left(\left[\rho_{s}(v)\right], \xi_{v, s}\right) \in \mathfrak{G}_{s}^{\perp}\right|_{\left[\rho_{s}(v)\right]}
$$

and

$$
\mathcal{D}_{h} \widetilde{b}_{s}\left(\tilde{\mu}_{s+1}\left(\varphi\left(b, \xi_{v, s}\right), v\right)\right)=\left\{\alpha_{s ; h}(b)\right\}\left(\rho_{s ; h}(v)\right) \quad \forall h \in \mathcal{I}_{s-1}^{*} .
$$

Furthermore, $\xi_{v, s}$ satisfies the first estimate in (6-26), with $t=s$, for some $C \in$ $C\left(\mathcal{U}_{\mathcal{T}}(X ; J) ; \mathbb{R}^{+}\right)$. The second estimate is obtained by differentiating (6-27). Thus, we take

$$
\mu_{s}(v)=\mu_{s+1}\left(\varphi\left(b, \xi_{v, s}\right), v\right) .
$$

Suppose we have defined $\mu_{s}(v)$ for all $s \in[\langle\mathcal{T}\rangle]$. By (6-23), (6-24), their proof, and (6-26),

$$
\pi_{-}^{0,1} b_{0}\left(\tilde{\mu}_{1}(v), \zeta\right)=\left\{\alpha_{0}(b)+\varepsilon_{0}(v, \zeta)\right\}(\rho(v)),
$$

where $\left.\varepsilon_{0}(v, \zeta) \in \gamma_{0}^{*} \otimes V_{0}\right|_{[\rho(v)]}$ satisfies

$$
\begin{aligned}
& \left\|\varepsilon_{0}(v, \zeta)\right\|,\left\|\nabla^{\mathcal{T}} \varepsilon_{0}(v, \zeta)\right\| \\
& \quad \leq C(b)\left(|v|^{1 / p}+|v|^{(p-2) / p}+\|\zeta\|_{v, p, 1}\right) \cdot\left(1+|\rho(v)|^{-1}\|\zeta\|_{\tilde{\mu}_{1}(v), p, 1}\right)
\end{aligned}
$$

if $\zeta \in \Gamma\left(\tilde{\mu}_{1}(v)\right)$ and $\|\zeta\|_{\tilde{\mu}_{1}(v), p, 1} \leq \delta(b)$. Thus, for every

$v=(b, v) \in \tilde{\mathcal{F}}^{1} \mathcal{T}_{\delta}^{\varnothing} \quad$ and $\quad \zeta \in \Gamma_{+}\left(\tilde{\mu}_{1}(v)\right)$ such that $\|\zeta\|_{\tilde{\mu}_{1}(v), p, 1} \leq 2 \widetilde{C}(b)|\rho(v)|$,

the equation

$$
\pi_{-}^{0,1} b_{0}\left(\tilde{\mu}_{1}(\varphi(b, \xi), v), \zeta\right)=\left\{\alpha_{0}(b)\right\}(\rho(v)) \equiv 0
$$


has a unique small solution $\xi_{v, 0}(\zeta) \in \widetilde{\Gamma}_{-}(b)$ such that

$$
\left.\left([\rho(v)], \xi_{v, 0}(\zeta)\right) \in \mathfrak{G}_{0}^{\perp}\right|_{[\rho(v)]},
$$

provided that

$$
C(b)\left(|v|^{1 / p}+|v|^{(p-2) / p}+\widetilde{C}(b)|\rho(v)|\right) \cdot(1+2 \widetilde{C}(b)) \leq C(b) \varepsilon(b) .
$$

Furthermore, this solution satisfies

$$
\left|\xi_{v, 0}(\zeta)\right| \leq C(b)\left(|v|^{1 / p}+|v|^{(p-2) / p}\right) \cdot\left(1+|\rho(v)|^{-1}\|\zeta\|_{\tilde{\mu}_{1}(v), p, 1}\right) .
$$

We put

$$
\mu_{0}(v, \zeta)=\mu_{1}\left(\varphi\left(b, \xi_{v, 0}(\zeta)\right), v\right) \quad \text { and } \quad \tilde{\mu}_{0}(v, \zeta)=\left(\mu_{0}(v, \zeta), v\right)
$$

Let $\mu_{0}(v)=\mu_{0}(v, 0)$ and $\tilde{\mu}_{0}(v, 0)=\tilde{\mu}_{0}(v, 0)$.

For every $v=(b, v)$ in $\widetilde{\mathcal{F}}^{1} \mathcal{T}^{\varnothing}$ sufficiently small, we define the map

$$
\Psi_{v}:\left\{\zeta \in \Gamma_{+}\left(\tilde{\mu}_{0}(v)\right):\|\zeta\|_{\tilde{\mu}_{0}(v), p, 1} \leq 2 \widetilde{C}(b)|\rho(v)|\right\} \longrightarrow \Gamma_{+}^{0,1}\left(\tilde{\mu}_{0}(v) ; J\right)
$$

by

$$
\Psi_{v}(\zeta)=\bar{\partial}_{J} u_{\tilde{\mu}_{0}(v, \zeta)}+D_{J, b_{0}\left(\mu_{0}(v, \zeta)\right)} \zeta+N_{J, \mu_{0}(v, \zeta)} \zeta \text {. }
$$

Let

$$
D_{J, v}^{+}: \Gamma_{+}\left(\tilde{\mu}_{0}(v)\right) \longrightarrow \Gamma_{+}^{0,1}\left(\tilde{\mu}_{0}(v) ; J\right)
$$

be the derivative of $\Psi_{v}$ at $\zeta=0$ and let $N_{J, v}^{+} \zeta \in \Gamma_{+}^{0,1}\left(\tilde{\mu}_{0}(v) ; J\right)$ be given by

$$
\Psi_{v}(\zeta)=\Psi_{v}(0)+D_{J, v}^{+} \zeta+N_{J, v}^{+} \zeta .
$$

By the construction of $\Psi_{v}$, (3) of Lemma 6.1, (4-27), and (6-32)

$$
\begin{aligned}
\left\|\Psi_{v}(0)\right\|_{\tilde{\mu}_{0}(v), p, 1} \leq 2 C(b)|\rho(v)| & \\
(2 C(b))^{-1}\|\zeta\|_{\tilde{\mu}_{0}(v), p, 1} & \leq\left\|D_{J, v}^{+} \zeta\right\|_{\tilde{\mu}_{0}(v), p} \\
& \leq 2 C(b)\|\zeta\|_{\tilde{\mu}_{0}(v), p, 1} \quad \forall \zeta \in \Gamma_{+}\left(\tilde{\mu}_{0}(v)\right),
\end{aligned}
$$

$$
\begin{aligned}
& \left\|N_{J, v}^{+} \zeta-N_{J, v}^{+} \zeta^{\prime}\right\|_{\tilde{\mu}_{0}((v), p} \\
& \leq 2 C(b)\left(\|\zeta\|_{\tilde{\mu}_{0}(v), p, 1}+\left\|\zeta^{\prime}\right\|_{\tilde{\mu}_{0}(v), p, 1}\right)\left\|\zeta-\zeta^{\prime}\right\|_{\tilde{\mu}_{0}(v), p, 1} \forall \zeta, \zeta^{\prime} \in \operatorname{Dom} \Psi_{v},
\end{aligned}
$$

provided that $v$ is sufficiently small. Since the index of $D_{J, v}^{+}$is zero, if $\tilde{C} \in$ $C\left(\mathcal{U}_{\mathcal{T}}(X ; J) ; \mathbb{R}^{+}\right)$is sufficiently large and $v \in \mathcal{F}^{1} \mathcal{T}_{\delta}^{1}$ is sufficiently small, by (6-33)(6-35) and the Contraction Principle, the equation

$$
\Psi_{v}(\zeta)=0
$$

has a unique solution $\zeta_{v, 0} \in \Gamma_{+}\left(\tilde{\mu}_{0}(v)\right)$. 
If $\tilde{\delta} \in C\left(\mathcal{U}_{\mathcal{T}}(X ; J) ; \mathbb{R}^{+}\right)$is sufficiently small, we define the map

(6-36) $\phi: \tilde{\mathcal{F}}^{1} \mathcal{T}_{\tilde{\delta}}^{\varnothing} \longrightarrow \mathfrak{M}_{1, M}^{0}(X, A ; J) \quad$ by $\quad \phi(v)=\left[\exp _{b_{0}\left(\tilde{\mu}_{0}\left(v, \zeta_{v, 0}\right)\right)} \zeta_{v, 0}\right]$

By construction, the map $\phi$ is $\operatorname{Aut}(\mathcal{T}) \propto\left(S^{1}\right)^{\hat{I}}$-invariant and smooth, and thus descends to a smooth map

$$
\phi: \mathcal{F}^{1} \mathcal{T}_{\tilde{\delta}}^{\varnothing} \longrightarrow \mathfrak{M}_{1, M}^{0}(X, A ; J) .
$$

By an argument analogous to that in Section 4.2 of [16], the map $\phi$ is an immersion into $\mathfrak{X}_{1, M}^{0}(X, A)$, if $\widetilde{\delta}$ is sufficiently small. By the proof of Corollary 4.6 and the construction of the map $\phi$, the image of $\phi$ contains $\mathfrak{M}_{1, M}^{0}(X, A ; J) \cap U_{\mathcal{T}}$ for a neighborhood $U_{\mathcal{T}}$ of $\mathcal{U}_{\mathcal{T}}(X ; J)$ in $\mathfrak{X}_{1, M}(X, A)$. Thus, the map

$$
\phi: \mathcal{F}^{1} \mathcal{T}_{\widetilde{\delta}}^{\varnothing} \longrightarrow \mathfrak{M}_{1, M}^{0}(X, A ; J) \cap U_{\mathcal{T}}
$$

is a diffeomorphism. It can be seen to be orientation-preserving by an argument similar to that of Section 3.9 in [16].

\subsection{Extension to homeomorphism}

In the rest of this section, we show that the map $\phi$ extends continuously over $\widetilde{\mathcal{F}}^{1} \mathcal{T}_{\widetilde{\delta}}$. This will be achieved by combining the approach of Sections 3.9 and 4.1 in [16] with the conditions (6-25) and (6-29) on the corrections $\xi_{s}(v)$ to the maps $\tilde{b}_{s}(v)$.

For every $b \in \tilde{\mathcal{U}}_{\mathcal{T}}^{(0)}(X ; J), s \in[\langle\mathcal{T}\rangle]$, and $h \in \mathcal{I}_{s-1}^{*}$, let

$$
\begin{gathered}
\Sigma_{b}^{h}=\bigcup_{h \leq i} \Sigma_{b, i} \subset \Sigma_{b}, \quad \Gamma_{h}^{0,1}(b ; J)=\left\{\eta \in \Gamma_{B}^{0,1}(b ; J):\left.\eta\right|_{\Sigma_{b}-\Sigma_{b}^{h}}=0\right\}, \\
\Gamma_{h}(b)=\left\{\xi \in \Gamma_{B}(b):\left.\xi\right|_{\Sigma_{b}-\Sigma_{b}^{h}}=0\right\}, \quad \widetilde{\Gamma}_{h ;-}(b)=\Gamma_{h}(b) \cap \widetilde{\Gamma}_{-}(b) .
\end{gathered}
$$

We note that by (a) of Definition 1.3, the operator

$$
D_{J, b}^{h}: \Gamma_{h}(b) \longrightarrow \Gamma_{h}^{0,1}(b ; J)
$$

induced by $D_{J, b}$ is surjective. By the regularity assumptions (a) and (b-i) of Definition 1.4 , the differential

$$
\nabla^{\mathcal{T}} \alpha_{s ; h}: \mathfrak{G}_{s ; h} \equiv \pi_{s ; h}^{*} \bigcup_{b \in \widetilde{\mathcal{U}}_{\mathcal{T}}^{(0)}(X ; J)} \widetilde{\Gamma}_{h ;-}(b) \longrightarrow \gamma_{s ; h}^{*} \otimes V_{s ; h}
$$


of $\alpha_{s ; h}$ is surjective. We denote by $\mathfrak{G}_{s ; h}^{\perp}$ be the $L^{2}$-orthogonal complement of $\operatorname{ker} \nabla^{\mathcal{T}} \alpha_{s ; h}$ in $\mathfrak{G}_{s ; h}$. If $\left.w_{h} \in \mathbb{P} \widetilde{\mathfrak{F}_{h} \mathcal{T}}\right|_{b}$ and $\left.w \in \mathbb{P}_{s} \mathcal{T}\right|_{b}$, let

$$
\begin{aligned}
\widetilde{\Gamma}_{h ;-}\left(b ; w_{h}\right) & =\left\{\xi \in \widetilde{\Gamma}_{h ;-}(b):\left(w_{h}, \xi\right) \in \mathfrak{G}_{s, h}^{\perp}\right\}, \\
\widetilde{\Gamma}_{-}(b ; w) & =\left\{\xi \in \widetilde{\Gamma}_{-}(b):(w, \xi) \in \mathfrak{G}_{s}^{\perp}\right\} .
\end{aligned}
$$

We note that

$$
\widetilde{\Gamma}_{-}(b ; w)=\bigoplus_{h \in \mathcal{I}_{s-1}^{*}} \widetilde{\Gamma}_{h ;-}\left(b ; w_{h}\right) \quad \text { if } \quad w=\left(w_{h}\right)_{h \in \mathcal{I}_{s-1}^{*}} .
$$

If $v=(b, v) \in \widetilde{\mathcal{F} \mathcal{T}}$ and $s$ and $h$ are as above, let

$$
\mathcal{I}_{s-1}^{0}(v)=\left\{h \in \mathcal{I}_{s-1}: \rho_{s ; h}(v)=0\right\} \quad \text { and } \quad \Sigma_{v_{\langle s\rangle}}^{h}=q_{v_{\langle s\rangle}}^{-1}\left(\Sigma_{b}^{h}\right) .
$$

We note that $\Sigma_{v_{\langle s\rangle}}^{h}$ is a union of components of $\Sigma_{v_{\langle s\rangle}}$.

The multistep gluing construction of Section 6.1 extends continuously over $\widetilde{\mathcal{F}}_{\delta}$. This extension is formally described in exactly the same way as the construction itself; see [16, Section 3.9] for a description of the continuous extension for a similar gluing construction and [16, Section 4.1] for a proof of its continuity. Using the notation of Section 6.1, we now make an observation regarding this extension. For each $s \in[\langle\mathcal{T}\rangle]$ and $h \in \mathcal{I}_{s-1}^{*}$, let

$$
\begin{gathered}
\Gamma_{h}\left(v_{\langle s\rangle}\right)=\left\{\xi \in \Gamma_{B}\left(v_{\langle s\rangle}\right):\left.\xi\right|_{\left.\Sigma_{v_{\langle s\rangle}}-\Sigma_{v_{\langle s\rangle}}^{h}=0\right\},}\right. \\
\Gamma_{h}^{0,1}\left(v_{\langle s\rangle} ; J\right)=\left\{\eta \in \Gamma_{B}^{0,1}\left(v_{\langle s\rangle} ; J\right):\left.\eta\right|_{\left.\Sigma_{v_{\langle s\rangle}}-\Sigma_{v_{\langle s\rangle}}^{h}=0\right\} .} .\right.
\end{gathered}
$$

By the surjectivity of the operators $D_{J, b}^{h}$, with $h \in \mathcal{I}_{s-1}^{*}$, for all $h \in \mathcal{I}_{s-1}^{*}$ the operators

$$
D_{J, b_{s}(v)}^{h}: \Gamma_{h}\left(v_{\langle s\rangle}\right) \longrightarrow \Gamma_{h}^{0,1}\left(v_{\langle s\rangle} ; J\right)
$$

induced by $D_{J, b_{s}(v)}$ are surjective, provided $v$ is sufficiently small. Since $\bar{\partial}_{J} u_{v, s}$ vanishes on $\Sigma_{v_{\langle s\rangle}}^{h}$ for all $h \in \mathcal{I}_{s-1}^{0}(v)$ and $\zeta_{v, s}$ is the unique small solution of (6-12), it follows that

$$
\left.\zeta_{v, s}\right|_{\Sigma_{v_{\langle s\rangle}}^{h}}=0 \forall h \in \mathcal{I}_{s-1}^{0}(v) .
$$

We next extend the construction of perturbations $\xi_{v, s}$ for $\left.v \in \widetilde{\mathcal{F} \mathcal{T}} \delta\right|_{\mathcal{U}_{\mathcal{T}}^{(s)}(X ; J)}$ in Section 6.2 to $\left.\widetilde{\mathcal{F}}_{\delta}\right|_{\mathcal{U}_{\mathcal{T}}^{(s)}(X ; J)}$. Suppose $s \in[\langle\mathcal{T}\rangle]$ and for all $t \in[\langle\mathcal{T}\rangle]$ such that $t>s$ and for all elements $v=(b, v)$ in $\left.\widetilde{\mathcal{F} \mathcal{T}} \delta\right|_{\mathcal{U}_{\mathcal{T}}^{(t)}(X ; J)}$ we have constructed

$$
\mu_{t}(v) \in \tilde{\mathcal{U}}_{\mathcal{T}}^{(0)}(X ; J) \quad \text { and } \quad \tilde{\mu}_{t}(v)=\left(\mu_{t}(v), v\right) \in \widetilde{\mathcal{F}}_{\delta}
$$


such that

$$
\begin{gathered}
\mathcal{D}_{h} \tilde{b}_{t}\left(\tilde{\mu}_{t}(v)\right)=\left\{\alpha_{t ; h}(b)\right\}\left(\rho_{h}(v)\right) \quad \forall h \in \mathcal{I}_{t-1}^{*}, \\
\mu_{t}(v)=\mu_{t+1}\left(\varphi\left(b, \xi_{v, t}\right), v\right)
\end{gathered}
$$

for some $\xi_{v, t} \in \bigoplus \widetilde{\Gamma}_{h ;-}\left(b ;\left[\rho_{h}(v)\right)\right.$ such that $\left|\xi_{v, t}\right|,\left|\nabla^{\mathcal{T}} \xi_{v, t}\right| \leq C(b)|v|^{1 / p}$

$$
h \in \mathcal{I}_{t-1}^{*}-\mathcal{I}_{t-1}^{0}(v)
$$

We note that $\mathcal{D}_{h} \widetilde{b}_{t}\left(\tilde{\mu}_{t}(v)\right)=0$ for any $h \in \mathcal{I}_{t-1}^{0}(v)$ and $\mu_{t}(v) \in \tilde{\mathcal{U}}_{\mathcal{T}}^{(0)}(X ; J)$. Thus, (6-39) is a nontrivial condition only for $h \in \mathcal{I}_{t-1}^{*}-\mathcal{I}_{t-1}^{0}(v)$. In particular, if $\mathcal{I}_{s-1}^{0}(v)=$ $\mathcal{I}_{s-1}^{*}$, for the inductive step in the construction of the previous subsection we simply take $\xi_{v, s}=0$. On the other hand, if $\mathcal{I}_{s-1}^{0}(v) \neq \mathcal{I}_{s-1}^{*}$, the inductive step is nearly the same as the before. The only difference is that instead of working with the section

$$
\alpha_{s} \equiv\left\{\alpha_{s ; h}\right\}_{h \in \mathcal{I}_{s-1}^{*}}
$$

over $\mathbb{P}_{s} \mathcal{T}$, we work with the section $\left\{\alpha_{s ; h}\right\}_{h \in \mathcal{I}_{s-1}^{*}-\mathcal{I}_{s-1}^{0}(v)}$ over the fiber product of the bundles

$$
\left\{\mathbb{P} \widetilde{\mathfrak{F}_{h} \mathcal{T}}\right\}_{h \in \mathcal{I}_{s-1}^{*}}-\mathcal{I}_{s-1}^{0}(v) \longrightarrow \tilde{\mathcal{U}}_{\mathcal{T}}^{(s+1)}(X ; J) .
$$

We note that in this case the orthogonal complement of the kernel of

$$
\nabla^{\mathcal{T}}\left\{\alpha_{s ; h}\right\}_{h \in \mathcal{I}_{s-1}^{*}-\mathcal{I}_{s-1}^{0}(v)}
$$

is given by (6-37) with $\mathcal{I}_{s-1}^{*}$ replaced by $\mathcal{I}_{s-1}^{*}-\mathcal{I}_{s-1}^{0}(v)$. In summary,

$$
\xi_{v, s} \in \bigoplus_{h \in \mathcal{I}_{s-1}^{*}-\mathcal{I}_{s-1}^{0}(v)} \widetilde{\Gamma}_{h ;-}\left(b ;\left[\rho_{s ; h}(v)\right)\right.
$$

is the unique small solution to the system of equations

$$
\alpha_{s ; h}\left(\varphi\left(b, \xi_{v, s}\right), w_{h}\right)+\varepsilon_{s ; h}\left(\varphi\left(b, \xi_{v, s}\right), v\right)=\alpha_{s ; h}\left(b, w_{h}\right), \quad h \in \mathcal{I}_{s-1}^{*}-\mathcal{I}_{s-1}^{0}(v) .
$$

The final, $s=0$, step splits into two cases as well. If $\rho(v)=0$, then we take

$$
\zeta_{v, 0}=0 \quad \text { and } \quad \xi_{v, 0} \equiv \xi_{v, 0}\left(\zeta_{v, 0}\right)=0 .
$$

Otherwise, the argument of the previous subsection still applies.

We will show that the above extension of the construction described in Section 6.2 is continuous at every step. First, note that by definition, for every $s \in[\langle\mathcal{T}\rangle-1]$ and $h \in \mathcal{I}_{s-1}^{*} \cap \chi^{-}(\mathcal{T})$

$$
\rho_{s ; h}(v)=\left(v_{h^{\prime}} \rho_{s+1 ; h^{\prime}}(v)\right)_{\iota_{h^{\prime}}=h} \quad \forall v \equiv\left(b,\left(v_{i}\right)_{i \in \mathbb{N} \cup \hat{I}}\right) \in \widetilde{\mathcal{F} \mathcal{T}}
$$


In this case, by the proof of Lemma 3.5,

$(6-42) \quad \mathcal{D}_{h} \tilde{b}_{s}(v)=\sum_{\iota_{h^{\prime}}=h} v_{h^{\prime}} \mathcal{D}_{h^{\prime}} \tilde{b}_{s+1}(v)+\sum_{i \in \chi_{h}(\mathcal{T})} \varepsilon_{h ; i}(v) \rho_{h ; i}(v)$ $\forall v \equiv(b, v) \in \widetilde{\mathcal{F}}_{\delta}^{\varnothing}$,

where $\varepsilon_{h ; i}(v) \in T_{\operatorname{ev}_{P}(b)} X$ is given by the right-hand side of (3-15), with

$$
k=1, \quad \widetilde{\zeta}_{\widetilde{J}, v}=\zeta_{v, s}, \quad \vartheta_{b}=\vartheta_{\widetilde{b}_{s+1}(v)}, \quad \Phi_{b}=\Phi_{J, v_{\langle s+1\rangle}}, \quad \Phi_{\widetilde{J}, v}=\Phi_{J, v_{\langle s\rangle}},
$$

and with $\delta_{K}$ replaced by a function $\delta=\delta(b)$. Since $\partial^{-} A_{i}^{-}(\delta(b)) \subset \Sigma_{v_{\langle s\rangle}}^{h}$ for all $i \in \chi_{h}(\mathcal{T})$, it follows that

$$
\begin{aligned}
&\left|\varepsilon_{h ; i}(v)\right|,\left|\nabla^{\mathcal{T}} \varepsilon_{h ; i}(v)\right| \leq C(b)\left(\left.\left\|\left.\zeta_{v, s}\right|_{\Sigma_{v\langle s\rangle}^{h}}\right\|\left\|_{v, p, 1}+\right\| \nabla^{\mathcal{T}} \zeta_{v, s}\right|_{\Sigma_{v\langle s\rangle}^{h}} \|_{v, p, 1}\right) \\
& \forall v \equiv(b, v) \in \widetilde{\mathcal{F}}_{\delta}^{\varnothing}, h \in \mathcal{I}_{s-1}^{*} \cap \chi^{-}(\mathcal{T}), i \in \chi_{h}(\mathcal{T}) .
\end{aligned}
$$

Let $\varepsilon_{s ; h}=\varepsilon_{s ; h}(v)$ be the bundle map as in (6-27). Combining the last estimate with (6-42), (6-41), and (6-25) with $t=s+1$, we find that

$$
\begin{aligned}
\left|\varepsilon_{s ; h}(v)\right|,\left|\nabla^{\mathcal{T}} \varepsilon_{s ; h}(v)\right| \leq C(b) & \left(\left\|\left.\zeta_{v, s}\right|_{\Sigma_{v_{\langle s\rangle}^{h}}^{h}}\right\|_{v, p, 1}+\left\|\left.\nabla^{\mathcal{T}} \zeta_{v, s}\right|_{\Sigma_{v_{\langle s\rangle}}^{h}}\right\|_{v, p, 1}\right) \\
& \left.\forall v \equiv(b, v) \in \widetilde{\mathcal{F}}_{\delta}^{\varnothing}\right|_{\mathcal{U}_{\mathcal{T}}^{(s+1)}(X ; J)}, h \in \mathcal{I}_{s-1}^{*}
\end{aligned}
$$

We note that if $h \in \mathcal{I}_{s-1}^{*}-\chi^{-}(\mathcal{T})$, (6-43) follows immediately from (6-25) with $t=s+1$.

We next observe that by the proof of the estimate in (6) of Lemma 4.4,

$$
\pi_{v ;-}^{0,1} \bar{\partial}_{J} u_{v, 0}=\sum_{h \in\left(\chi(\mathcal{T}) \cup \chi^{-}(\mathcal{T})\right) \cap I_{1}}\left(-2 \pi \mathfrak{i} v_{h} \mathcal{D}_{h} \tilde{b}_{1}(v)+\varepsilon_{h}(v)+\widetilde{\varepsilon}_{h}(v)\right)
$$

for all $v \equiv(b, v) \in \widetilde{\mathcal{F}}_{\delta}^{\varnothing}$. The error term $\varepsilon_{h}(v)$ is described by the left-hand side of (4-22). This term and its $\nabla^{\mathcal{T}}$-derivative are bounded by the last expression in (4-25). The other term is given by

$$
\tilde{\varepsilon}_{h}(v)=v_{h}\left(2 \pi \mathfrak{i} \mathcal{D}_{h} \tilde{b}_{1}(v)-\oint_{\partial A_{v_{\langle 1\rangle}, h}^{-}\left(\left|v_{h}\right|^{2} / \delta(b)\right)} \tilde{\zeta}_{b ; v} \frac{d w_{h}}{w_{h}^{2}}\right),
$$

where $\tilde{\zeta}_{b ; v}: A_{v_{\langle 1\rangle}, h}^{-}(\delta(b)) \longrightarrow T_{\operatorname{ev}_{P}(b)} X, \quad \exp _{\operatorname{ev}_{P}(b)} \tilde{\zeta}_{b ; v}=\tilde{u}_{v, 1}, \quad\left\|\tilde{\zeta}_{b ; v}\right\|_{C^{0}}<r_{J}$ see the proof of (6) of Lemma 4.4. Applying the approach of Lemma 3.3 and Cauchy's formula, we find that

$$
\tilde{\varepsilon}_{h}(v)=v_{h} \oint_{\partial A_{v_{\langle 1\rangle}, h}^{-}\left(\left|v_{h}\right|^{2} / \delta(b)\right)}\left(\widetilde{\Phi}_{v, 1}-\mathrm{id}\right) \tilde{\vartheta}_{v, 1} \frac{d w_{h}}{w_{h}^{2}},
$$


where $\left.\widetilde{\Phi}_{v, 1}\right|_{w=0}=\mathrm{id}$,

$$
\begin{gathered}
\left\|\widetilde{\Phi}_{v, 1}-\mathrm{id}\right\|_{v_{1}, p, 1} \leq C(b)\left\|\left.d u_{v, 1}\right|_{A_{v_{\langle 1\rangle}, h}(\delta(b))}\right\|_{v_{1}, p, 1} \leq C(b)\left|\tilde{\rho}_{h}(v)\right|, \\
\left|\widetilde{\vartheta}_{v, 1}\right|_{w_{h}} \leq C(b)\left|w_{h}\right|\left|\widetilde{\rho}_{h}(v)\right|,
\end{gathered}
$$

by (2b) and the first estimate in (2c) of Corollary 3.8. By (6-45)-(6-47) and Holder's inequality

$$
\begin{aligned}
\left|\widetilde{\varepsilon}_{h}(v)\right| & \leq C(b)\left|v_{h}\right|\left|\tilde{\rho}_{h}(v)\right| \int_{0}^{2 \pi}\left|\widetilde{\Phi}_{v, 1}\left(\left|v_{h}\right|^{2} / \delta(b), \theta\right)-\mathrm{id}\right| d \theta \\
& \leq C(b)\left|\rho_{h}(v)\right| \int_{0}^{2 \pi} \int_{0}^{\left|v_{h}\right|^{2} / \delta(b)}\left|d \widetilde{\Phi}_{v, 1}\right|_{r, \theta} d r d \theta \\
& \leq C(b)|\rho(v)|\left\|\widetilde{\Phi}_{v, 1}-\mathrm{id}\right\|_{v_{1}, p, 1}\left(\int_{A_{v_{\langle 1\rangle}, h}\left(\left|v_{h}\right|^{2} / \delta(b)\right)}^{\left|w_{h}\right|^{-p /(p-1)}}\right)^{(p-1) / p} \\
& \leq C(b)|\rho(v)|\left|\widetilde{\rho}_{h}(v)\right| \cdot\left|v_{h}\right|^{(p-2) / p} \leq C(b)|\rho(v)|^{p-2 / p}|\rho(v)| .
\end{aligned}
$$

Let $\varepsilon_{0}=\varepsilon_{0}(v, \zeta)$ be the bundle map as in (6-29). Combining the last estimate with (6-42), (6-44), (6-25) with $t=1$, and (4) of Lemma 6.1 we find that

$$
\begin{aligned}
& \left|\varepsilon_{0}(v, \zeta)\right|,\left|\nabla^{\mathcal{T}} \varepsilon_{0}(v, \zeta)\right| \leq C(b)|\rho(v)|^{(p-2) / p} \\
& \left.\forall v \equiv(b, v) \in \widetilde{\mathcal{F}}_{\delta}^{\varnothing}\right|_{\mathcal{U}_{\mathcal{T}}^{(1)}(X ; J)}, \zeta \in \Gamma(v) \text { such that }\|\zeta\|_{v, p, 1} \leq \widetilde{C}(b)|\rho(v)| .
\end{aligned}
$$

The same holds for the derivatives of $\varepsilon_{0}(v, \zeta)$ and $\nabla^{\mathcal{T}} \varepsilon_{0}(v, \zeta)$ with respect to $\zeta$.

Suppose $s \in[\langle\mathcal{T}\rangle]$ and for every $t \in[\langle\mathcal{T}\rangle]$ such that $t>s$ the bundle map

$$
\widetilde{\mathcal{F}}_{\delta} \longrightarrow \widetilde{\Gamma}_{-}(\cdot), \quad v \longrightarrow \xi_{v, t},
$$

and its $\nabla^{\mathcal{T}}$-derivative are continuous over $\mathcal{U}_{\mathcal{T}}^{(t)}(X ; J)$. We will show that this must also be the case for $t=s$. Since the maps

$$
\widetilde{\mathcal{F}}_{\delta} \longrightarrow \underbrace{}_{v \in \widetilde{\mathcal{F}}_{\delta}} \Gamma_{B ;+}\left(v_{\langle s\rangle}\right), \quad v \longrightarrow \zeta_{v, s},
$$

and

$$
\left.\widetilde{\mathcal{F}}_{\delta}\right|_{\mathcal{U}_{\mathcal{T}}^{(s+1)}(X ; J)} \longrightarrow \widetilde{\mathcal{F} \mathcal{T}}_{\delta}, \quad v \longrightarrow \tilde{\mu}_{s+1}(v),
$$

are continuous, so are the maps

$$
\left.\widetilde{\mathcal{F}}_{\delta}\right|_{\mathcal{U}_{\mathcal{T}}^{(s+1)}(X ; J)} \longrightarrow \gamma_{s ; h}^{*} \otimes V_{s ; h}, \quad v \longrightarrow \varepsilon_{s ; h}(v),
$$


for all $h \in \mathcal{I}_{s-1}^{*}$. Suppose $v_{r} \equiv\left(b_{r}, v_{r}\right)$ is a sequence of elements in $\left.\widetilde{\mathcal{F}}_{\delta}^{\varnothing}\right|_{\mathcal{U}_{\mathcal{T}}^{(s)}(X ; J)}$ such that

$$
\lim _{r \longrightarrow \infty} b_{r}=b^{\prime} \in \mathcal{U}_{\mathcal{T}}^{(s)}(X ; J) \quad \text { and } \quad \lim _{r \longrightarrow \infty} v_{r}=v^{\prime} \equiv\left(b^{\prime}, v^{\prime}\right) \in \widetilde{\mathcal{F}}_{\delta} .
$$

Let

$$
\begin{gathered}
w_{h}=\left.\lim _{r \longrightarrow \infty}\left[\rho_{s ; h}\left(v_{r}\right)\right] \in \mathbb{P} \widetilde{\mathfrak{F}_{h} \mathcal{T}}\right|_{b^{\prime}} \quad \text { if } h \in \mathcal{I}_{s-1}^{*} ; \quad w=\left.\left(w_{h}\right)_{h \in \mathcal{I}_{s-1}^{*}} \in \mathbb{P}_{s} \mathcal{T}\right|_{b^{\prime}} ; \\
\xi_{v^{\prime}, s}^{\prime} \equiv\left(\xi_{v^{\prime}, s ; h}^{\prime}\right)_{h \in \mathcal{I}_{s-1}^{*}}=\lim _{r \longrightarrow \infty} \xi_{v_{r}, s} \in \widetilde{\Gamma}_{-}\left(b^{\prime} ; w\right)=\bigoplus_{h \in \mathcal{I}_{s-1}^{*}} \widetilde{\Gamma}_{h ;-}\left(b ; w_{h}\right) .
\end{gathered}
$$

We recall that

$$
\xi_{v_{r}, s} \equiv\left(\xi_{v_{r}, s ; h}\right)_{h \in \mathcal{I}_{s-1}^{*}} \in \widetilde{\Gamma}_{-}\left(b_{r} ;\left[\rho_{s}\left(v_{r}\right)\right]\right)=\bigoplus_{h \in \mathcal{I}_{s-1}^{*}} \widetilde{\Gamma}_{h ;-}\left(b ;\left[\rho_{s ; h}\left(v_{r}\right)\right]\right)
$$

is the unique small solution to the system of equations $\alpha_{s ; h}\left(\varphi\left(b, \xi_{v_{r}, s}\right),\left[\rho_{s ; h}\left(v_{r}\right)\right]\right)+\varepsilon_{s ; h}\left(\varphi\left(b, \xi_{v_{r}, s}\right), v_{r}\right)=\alpha_{s ; h}\left(b,\left[\rho_{s ; h}\left(v_{r}\right)\right]\right), \quad h \in \mathcal{I}_{s-1}^{*}$. Thus, $\xi_{v^{\prime}, s}^{\prime} \in \widetilde{\Gamma}_{-}\left(b^{\prime} ; w\right)$ is the unique small solution to the system of equations

$$
\alpha_{s ; h}\left(\varphi\left(b, \xi_{v^{\prime}, s}^{\prime}\right), w_{h}\right)+\varepsilon_{s ; h}\left(\varphi\left(b, \xi_{v^{\prime}, s}^{\prime}\right), v^{\prime}\right)=\alpha_{s ; h}\left(b, w_{h}\right), \quad h \in \mathcal{I}_{s-1}^{*} .
$$

Since

$$
\varepsilon_{s ; h}\left(\varphi\left(b, \xi_{v^{\prime}, s}^{\prime}\right), v^{\prime}\right)=0 \quad \forall h \in \mathcal{I}_{s-1}^{0}\left(v^{\prime}\right)
$$

by (6-43) and (6-38), $\xi_{v^{\prime}, s ; h}^{\prime}=0$ for all $h \in \mathcal{I}_{s-1}^{0}\left(v^{\prime}\right)$ and $\xi_{v^{\prime}, s}^{\prime}=\xi_{v^{\prime}, s}$, as needed.

We finally show that the map

$$
v \longrightarrow \widetilde{b}_{0}(v) \equiv\left(\Sigma_{v} ; \exp _{b_{0}\left(\tilde{\mu}_{0}\left(v, \zeta_{v, 0}\right)\right)} \zeta_{v, 0}\right)
$$

is continuous over $\widetilde{\mathcal{F}}^{1} \mathcal{T}_{\delta}^{\varnothing}$. First, the map

$$
\bigcup_{v \in \widetilde{\mathcal{F}}_{\delta}, \rho(v) \neq 0}^{\bigcup}\left\{\zeta \in \Gamma_{+}(v):\|\zeta\|_{v, p, 1}<\delta(b)\right\} \rightarrow \bigcup_{b \in \tilde{\mathcal{U}}_{\mathcal{T}}^{(0)}(X ; J)} \Gamma_{-}^{0,1}(b ; J), \quad(v, \zeta) \longrightarrow \varepsilon(v, \zeta),
$$

of (6-23) is continuous. Since so is the map $v \longrightarrow \xi_{v, 1}$, the map

$v \in \widetilde{\mathcal{F}}_{\delta}, \rho(v) \neq 0$

$$
\bigcup\left\{\zeta \in \Gamma_{+}(v):\|\zeta\|_{v, p, 1}<\widetilde{C}(b)|\rho(v)|\right\} \longrightarrow \gamma_{0}^{*} \otimes V_{0}, \quad(v, \zeta) \longrightarrow \varepsilon_{0}(v, \zeta),
$$

is also continuous. It then follows immediately from the construction that the maps

$$
(v, \zeta) \longrightarrow \xi_{v, 0}(\zeta) \quad \text { and } \quad v \longrightarrow \zeta_{v, 0}
$$


are continuous over $\widetilde{\mathcal{F}}^{1} \mathcal{T}_{\delta}-\rho^{-1}(0)$. On the other hand, suppose $v_{r} \equiv\left(b_{r}, v_{r}\right)$ is a sequence of elements in $\widetilde{\mathcal{F}}^{1} \mathcal{T}_{\delta}^{\varnothing}$ such that

$\lim _{r \longrightarrow \infty} b_{r}=b^{\prime} \in \mathcal{U}_{\mathcal{T}}^{(0)}(X ; J), \quad \lim _{r \longrightarrow \infty} v_{r}=v^{\prime} \equiv\left(b^{\prime}, v^{\prime}\right) \in \widetilde{\mathcal{F}}^{1} \mathcal{T}_{\delta} \quad$ and $\quad \rho\left(v^{\prime}\right)=0$

Let

$$
w=\left.\lim _{r \rightarrow \infty}\left[\rho\left(v_{r}\right)\right] \in \mathbb{P} \widetilde{\mathfrak{F} \mathcal{T}}\right|_{b^{\prime}} \quad \text { and } \quad \xi_{v^{\prime}, 0}^{\prime}=\lim _{r \rightarrow \infty} \xi_{v_{r}, 0}\left(\zeta_{v_{r}, 0}\right) \in \widetilde{\Gamma}_{-}\left(b^{\prime} ; w\right) .
$$

Since $\rho\left(v_{r}\right) \longrightarrow 0$, (6-49) implies that $\xi_{v^{\prime}, 0}^{\prime} \in \widetilde{\Gamma}_{-}\left(b^{\prime} ; w\right)$ is the unique small solution of

$$
\alpha_{0}\left(\varphi\left(b^{\prime}, \xi_{v^{\prime}, 0}^{\prime}\right), w\right)=0=\alpha_{0}\left(b^{\prime}, w\right) .
$$

Thus, $\xi_{v^{\prime}, 0}^{\prime}=0=\xi_{v^{\prime}, 0}$. Furthermore, by (6-22)

$$
\lim _{r \rightarrow \infty} \zeta_{v_{r}, 0}=0=\zeta_{v^{\prime}, 0}
$$

It follows that the map (6-50) is continuous.

We have thus constructed a continuous map

$$
\phi: \mathcal{F}^{1} \mathcal{T}_{\delta} \longrightarrow \overline{\mathfrak{M}}_{1, M}^{0}(X, A ; J) \cap U_{\mathcal{T}},
$$

where $U_{\mathcal{T}}$ is a neighborhood of $\mathcal{U}_{\mathcal{T} ; 1}(X ; J)$ in $\mathfrak{X}_{1, M}(X, A)$. By the same argument as in Sections 4.2 and 4.5 of [17], this map is injective if $\delta \in C\left(\mathcal{U}_{\mathcal{T}}(X ; J) ; \mathbb{R}^{+}\right)$and surjective if $U_{\mathcal{T}}$ is sufficiently small. Since the space $\overline{\mathfrak{M}}_{1, M}^{0}(X, A ; J)$ is Hausdorff and $\left.\phi\right|_{\mathcal{U}_{\mathcal{T}}(X ; J)}$ is the identity map, it follows that $\phi$ is a homeomorphism for $\delta$ sufficiently small.

\section{References}

[1] K Behrend, B Fantechi, The intrinsic normal cone, Invent. Math. 128 (1997) 45-88 MR1437495

[2] M Bershadsky, S Cecotti, H Ooguri, C Vafa, Holomorphic anomalies in topological field theories, Nuclear Phys. B 405 (1993) 279-304 MR1240687

[3] A Floer, H Hofer, D Salamon, Transversality in elliptic Morse theory for the symplectic action, Duke Math. J. 80 (1995) 251-292 MR1360618

[4] K Fukaya, K Ono, Arnold conjecture and Gromov-Witten invariant, Topology 38 (1999) 933-1048 MR1688434

[5] E Ionel, Genus 1 enumerative invariants in $\mathbf{P}^{n}$ with fixed $j$ invariant, Duke Math. J. 94 (1998) 279-324 MR1638587 
[6] J Li, G Tian, Virtual moduli cycles and Gromov-Witten invariants of general symplectic manifolds, from: "Topics in symplectic 4-manifolds (Irvine, CA, 1996)", (R J Stern, editor), First Int. Press Lect. Ser. I, Int. Press, Cambridge, MA (1998) 47-83 MR1635695

[7] J Li, A Zinger, On the genus-one Gromov-Witten invariants of complete intersections, to appear in J. Diff. Geom. arXiv:math/0507104

[8] D McDuff, D Salamon, J-holomorphic curves and quantum cohomology, Univ. Lecture Series 6, Amer. Math. Soc. (1994) MR1286255

[9] Y Ruan, G Tian, A mathematical theory of quantum cohomology, J. Differential Geom. 42 (1995) 259-367 MR1366548

[10] Y Ruan, G Tian, Higher genus symplectic invariants and sigma models coupled with gravity, Invent. Math. 130 (1997) 455-516 MR1483992

[11] R Vakil, A tool for stable reduction of curves on surfaces, from: "Advances in algebraic geometry motivated by physics (Lowell, MA, 2000)", (E Previato, editor), Contemp. Math. 276, Amer. Math. Soc. (2001) 145-154 MR1837115

[12] R Vakil, A Zinger, A desingularization of the main component of the moduli space of genus-one stable maps into $\mathbb{P}^{n}$, Geom. Topol. 12 (2008) 1-95 MR2377245

[13] A Zinger, Enumeration of genus-two curves with a fixed complex structure in $\mathbb{P}^{2}$ and $\mathbb{P}^{3}$, J. Differential Geom. 65 (2003) 341-467 MR2064428

[14] A Zinger, Basic estimates of riemannian geometry used in gluing pseudoholomorphic maps, Notes (2004) Available at http://www.math.sunysb.edu/ azinger/ research/analytic.pdf

[15] A Zinger, Completion of Katz-Qin-Ruan's enumeration of genus-two plane curves, J. Algebraic Geom. 13 (2004) 547-561 MR2047680

[16] A Zinger, Enumerative vs. symplectic invariants and obstruction bundles, J. Symplectic Geom. 2 (2004) 445-543 MR2197218

[17] A Zinger, Counting rational curves of arbitrary shape in projective spaces, Geom. Topol. 9 (2005) 571-697 MR2140990

[18] A Zinger, On the structure of certain natural cones over moduli spaces of genus-one holomorphic maps, Adv. Math. 214 (2007) 878-933 MR2349722

[19] A Zinger, Reduced genus-one Gromov-Witten invariants arXiv:math/0507103

[20] A Zinger, Pseudocycles and integral homology, Trans. Amer. Math. Soc. 360 (2008) 2741-2765 MR2373332

[21] A Zinger, Standard versus reduced genus-one Gromov-Witten invariants, Geom. Topol. 12 (2008) 1203-1241 MR2403808

[22] A Zinger, The reduced genus-one Gromov-Witten invariants of Calabi-Yau hypersurfaces, J. Amer. Math. Soc. 22 (2009) 691-737 
Department of Mathematics, SUNY Stony Brook Stony Brook, NY 11794-3651, USA

azinger@math. sunysb . edu

http://www . math. sunysb.edu/ azinger

Proposed: Gang Tian

Received: 7 August 2007

Seconded: Jim Bryan, Yasha Eliashberg

Revised: 7 December 2008 